Supporting Information

\title{
Enantioselective Ruthenium-Catalyzed C-H Alkylations by a Chiral Carboxylic Acid with Attractive Dispersive Interactions
}

\author{
Uttam Dhawa, ${ }^{\dagger}$ Robert Connon, ${ }^{\dagger}$ João C. A. Oliveira, ${ }^{\dagger}$ Ralf Steinbock ${ }^{\dagger}$ and Lutz Ackermann* ${ }^{\dagger} \S$
}

Institut für Organische und Biomolekulare Chemie

Georg-August-Universität

Tammannstraße 2, D-37077 Göttingen (Germany)

Fax: $+49 /$ 551-39-6777

${ }^{\S}$ Wöhler Research Institute for Sustainable Chemistry (WISCh)

Georg-August-Universität Göttingen

Tammannstraße 2, 37077 Göttingen (Germany)

*E-Mail: Lutz.Ackermann@ chemie.uni-goettingen.de 


\section{Table of Contents}

$\begin{array}{lr}\text { General Remarks } & \text { S3 }\end{array}$

$\begin{array}{lr}\text { Synthesis of Chiral Acids } & \text { S4 }\end{array}$

Optimization of the Reaction Conditions $\quad$ S8

General Procedure for the Ruthenium(II)-Catalyzed Asymmetric C-H Alkylation $\quad$ S10

$\begin{array}{ll}\text { Synthesis of Starting Materials } \mathbf{1} \text { and } \mathbf{3} & \text { S11 }\end{array}$

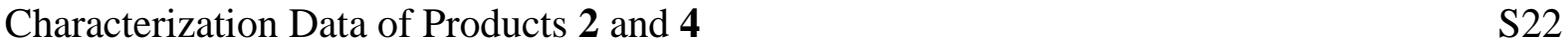

$\begin{array}{ll}\text { Unsucessful substrates } & \text { S48 }\end{array}$

$\begin{array}{lr}\text { H/D Exchange Experiment } & \text { S49 }\end{array}$

Intramolecular KIE Experiment $\quad$ S53

Determination of Absolute Stereochemistry $\quad$ S55

$\begin{array}{lr}\text { Computational Studies } & \text { S59 }\end{array}$

$\begin{array}{lr}\text { References } & \text { S84 }\end{array}$

$\begin{array}{ll}\text { NMR-Spectra } & \text { S87 }\end{array}$ 


\section{General Remarks}

Catalytic reactions were carried out in Schlenk flasks under $\mathrm{N}_{2}$ atmosphere using pre-dried glassware. Toluene was dried and distilled over $\mathrm{CaH}_{2}$ under $\mathrm{N}_{2}$. Chiral carboxylic acids CA1$\mathbf{C A 2}^{1}$ and $\mathbf{C A 4 - \mathbf { C A 5 } ^ { 2 }}{ }^{2}$ were prepared by previously reported methods. Other chemicals were obtained from commercial sources and were used without further purification. Yields refer to isolated compounds, estimated to be $>95 \%$ pure as determined by ${ }^{1} \mathrm{H}-\mathrm{NMR}$. TLC: MachereyNagel, TLC plates Alugram ${ }^{\circledR S i l ~ G / U V 254 . ~ D e t e c t i o n ~ u n d e r ~ U V ~ l i g h t ~ a t ~} 254 \mathrm{~nm}$. Chromatography: Separations were carried out on Merck Silica 60 (0.040-0.063 mm, 70-230 mesh ASTM). All IR spectra were recorded on a BRUKER ALPHA-P spectrometer. MS: EI-MS: Finnigan MAT 95, 70 eV; ESI-MS: Finnigan LCQ. High resolution mass spectrometry (HRMS): APEX IV 7T FTICR, Bruker Daltonic. M. p. Stuart® Melting Point Apparatus SMP3 melting point apparatus, values are uncorrected. HPLC chromatograms were recorded on an Agilent 1290 Infinity using CHIRALPAK® IB-3, ID-3 and IF-3 columns ( $3.0 \mu \mathrm{m}$ particle size; Ø: $4.6 \mathrm{~mm}$ and $250 \mathrm{~mm}$ length). Optical rotations were measured with Perkin Elmer 343 polarimeter at the stated temperature under a Na/Hg lamp, $\lambda=589 \mathrm{~nm}$ (c in g/100 ml). ${ }^{1} \mathrm{H},{ }^{13} \mathrm{C}$ and ${ }^{19} \mathrm{~F}$ NMR-spectra were recorded at 300 $\left({ }^{1} \mathrm{H}\right), 400\left({ }^{1} \mathrm{H}\right), 500\left({ }^{1} \mathrm{H}\right), 600\left({ }^{1} \mathrm{H}\right), 75,100,125\left[{ }^{13} \mathrm{C}\right.$, APT (Attached Proton Test) $]$ and 282, 377 $\mathrm{MHz}\left({ }^{19} \mathrm{~F}\right)$, respectively, on Varian Unity-300 (600) and AMX 300 instruments in $\mathrm{CDCl}_{3}$ or $\mathrm{CD}_{3} \mathrm{CO}_{2} \mathrm{D}$ solutions. If not otherwise specified, chemical shifts $(\delta)$ are given in ppm. 


\section{Synthesis of chiral acid CA6-CA10}

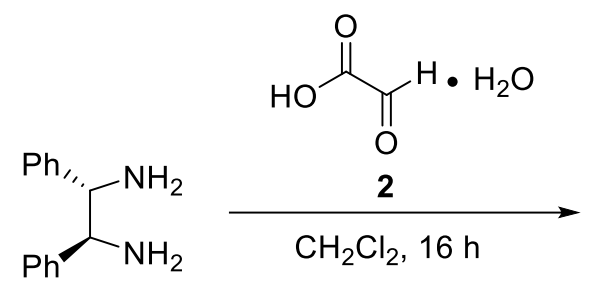

1

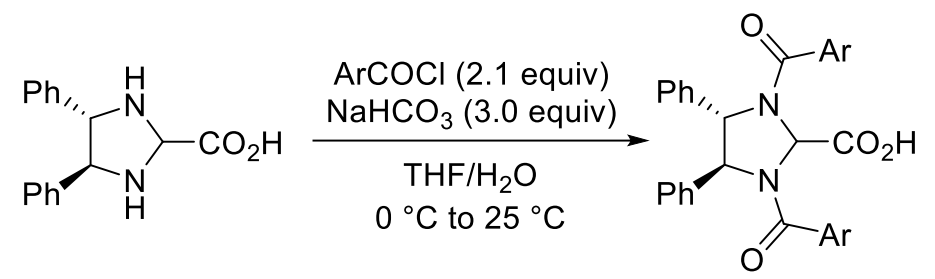

3

CA6-CA10

To a solution of $(1 S, 2 S)$-diphenylethanediamine $1(212.3 \mathrm{mg}, 1.00 \mathrm{mmol})$ in $\mathrm{CH}_{2} \mathrm{Cl}_{2}(12.0 \mathrm{~mL})$ was added glyoxylic acid monohydrate $2(92.1 \mathrm{mg}, 1.00 \mathrm{mmol})$ under vigorous stirring. ${ }^{3}$ After 16 $\mathrm{h}$, the solvent was removed to afford (4S,5S)-4,5-diphenylimidazolidine-2- carboxylic acid 3 (286.0 mg, 99\% yield) as a yellow solid. To a solution of 3 in $\mathrm{THF} / \mathrm{H}_{2} \mathrm{O}(5 \mathrm{~mL}, 1: 1)$ at $0{ }^{\circ} \mathrm{C}$, $\mathrm{NaHCO}_{3}$ (252.0 mg, $3.00 \mathrm{mmol}, 3$ equiv) and acyl chloride ( $2.10 \mathrm{mmol}, 2.1$ equiv) were added, and the reaction was allowed to reach ambient temperature. After $16 \mathrm{~h}$, the reaction mixture was diluted with $\mathrm{CH}_{2} \mathrm{Cl}_{2}(10 \mathrm{~mL})$ and washed with $\mathrm{HCl}(1 \mathrm{M}, 10 \mathrm{~mL})$ solution. The aqueous phase was extracted with $\mathrm{CH}_{2} \mathrm{Cl}_{2}(3 \times 10 \mathrm{~mL})$. The combined organic phase was washed with brine and dried over $\mathrm{Na}_{2} \mathrm{SO}_{4}$ and the solvent was removed in vacuo. The crude mixture was purified via flash chromatography $\left(\mathrm{CH}_{2} \mathrm{Cl}_{2} 100 \%\right.$ to $\left.\mathrm{CH}_{2} \mathrm{Cl}_{2}: \mathrm{CH}_{3} \mathrm{OH}=90: 10\right)$ to afford chiral acids CA6-CA10.<smiles>O=C(c1cccc(C(F)(F)F)c1)N1C(=O)N(C(=O)c2cccc(C(F)(F)F)c2)[C@H](c2ccccc2)C1c1ccccc1</smiles>

(4S,5S)-4,5-Diphenyl-1,3-bis[3-(trifluoromethyl)benzoyl]imidazolidine-2-carboxylic acid (CA6): General procedure for the preparation of chiral acid was followed using 3(trifluoromethyl)benzoyl chloride (436.8 mg, $2.10 \mathrm{mmol}$ ) to afford CA6 (367.1 $\mathrm{mg}, 60 \%$ yield) as a white solid. M.p.: $>200{ }^{\circ} \mathrm{C} .{ }^{1} \mathrm{H}-\mathrm{NMR}\left(600 \mathrm{MHz}, \mathrm{CD}_{3} \mathrm{CO}_{2} \mathrm{D}\right): \delta=7.62-7.48(\mathrm{~m}, 5 \mathrm{H}), 7.42-7.34$ (m, 2H), 7.29-7.13 (m, 9H), 7.11-6.99 (m, 2H), 6.98-6.83 (m, 1H), 5.35 (s, 1H), 5.17 (bs, 1H). ${ }^{13} \mathrm{C}-\mathrm{NMR}$ (100 MHz, $\mathrm{CD}_{3} \mathrm{CO}_{2} \mathrm{D}$ ) (one carbon resonances are missing due to overlap): $\delta=171.7$ 
$\left(\mathrm{C}_{\mathrm{q}}\right), 171.6\left(\mathrm{C}_{\mathrm{q}}\right), 170.9\left(\mathrm{C}_{\mathrm{q}}\right), 140.0\left(\mathrm{C}_{\mathrm{q}}\right), 138.7\left(\mathrm{C}_{\mathrm{q}}\right), 137.0\left(\mathrm{C}_{\mathrm{q}}\right), 136.1\left(\mathrm{C}_{\mathrm{q}}\right), 131.5\left(\mathrm{q},{ }^{2} J_{\mathrm{C}-\mathrm{F}}=32.4\right.$ $\left.\mathrm{Hz}, \mathrm{C}_{\mathrm{q}}\right), 131.4\left(\mathrm{q},{ }^{2} J_{\mathrm{C}-\mathrm{F}}=32.4 \mathrm{~Hz}, \mathrm{C}_{\mathrm{q}}\right), 131.4(\mathrm{CH}), 130.3(\mathrm{CH}), 130.2(\mathrm{CH}), 130.1(\mathrm{CH}), 129.9$ $(\mathrm{CH}), 129.5(\mathrm{CH}), 128.2(\mathrm{CH}), 128.2(\mathrm{CH}), 128.0(\mathrm{CH}), 127.6(\mathrm{CH}), 127.4(\mathrm{CH}), 124.9(\mathrm{CH})$, $124.9(\mathrm{CH}), 124.7\left(\mathrm{q},{ }^{1} J_{\mathrm{C}-\mathrm{F}}=271.8 \mathrm{~Hz}, \mathrm{C}_{\mathrm{q}}\right), 124.6\left(\mathrm{q},{ }^{1} J_{\mathrm{C}-\mathrm{F}}=271.8 \mathrm{~Hz}, \mathrm{C}_{\mathrm{q}}\right), 74.5(\mathrm{CH}), 73.3(\mathrm{CH})$, $71.6(\mathrm{CH}) .{ }^{19} \mathrm{~F}-\mathrm{NMR}\left(377 \mathrm{MHz}, \mathrm{CD}_{3} \mathrm{CO}_{2} \mathrm{D}\right): \delta=-63.7(\mathrm{~m}),-63.8(\mathrm{~m})$. IR (ATR): 3066, 3033, $1651,1456,1386,1326,1280,1121,1072,750,699 \mathrm{~cm}^{-1} \cdot[\alpha]_{\mathrm{D}}^{20}:-49.2\left(\mathrm{c}=1.00, \mathrm{CHCl}_{3}\right) . \mathrm{MS}$ (ESI-TOF) $m / z$ (relative intensity): $1247(20)[2 \mathrm{M}+\mathrm{Na}]^{+}, 635(100)[\mathrm{M}+\mathrm{Na}]^{+}, 613(15)[\mathrm{M}+$ $\mathrm{H}]^{+}$. HR-MS (ESI-TOF) $m / z:[\mathrm{M}+\mathrm{Na}]^{+}$Calcd. for $\mathrm{C}_{32} \mathrm{H}_{22}{ }^{19} \mathrm{~F}_{6} \mathrm{~N}_{2} \mathrm{O}_{4} \mathrm{Na}$ 635.1376; Found 635.1361.

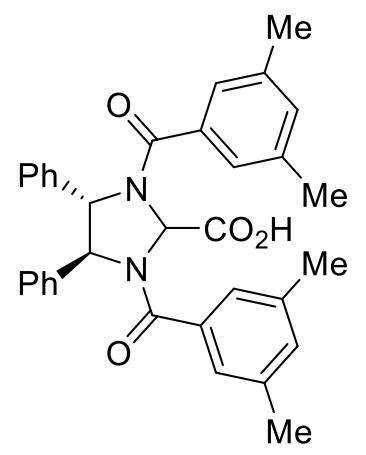

(4S,5S)-1,3-Bis(3,5-dimethylbenzoyl)-4,5-diphenylimidazolidine-2-carboxylic acid (CA7): General procedure for the preparation of chiral acid was followed using 3,5-dimethylbenzoyl chloride (352.8 mg, $2.10 \mathrm{mmol}$ ) to afford CA7 (329.0 mg, 62\% yield) as a white solid. M.p.: 180$186{ }^{\circ} \mathrm{C} .{ }^{1} \mathrm{H}-\mathrm{NMR}\left(400 \mathrm{MHz}, \mathrm{CD}_{3} \mathrm{CO}_{2} \mathrm{D}\right): \delta=7.46-7.27$ (m, 8H), 7.25-7.08 (m, 2H), 7.04-6.67 $(\mathrm{m}, 7 \mathrm{H}), 5.51(\mathrm{~s}, 1 \mathrm{H}), 5.24(\mathrm{bs}, 1 \mathrm{H}), 2.19(\mathrm{~s}, 6 \mathrm{H}), 2.14(\mathrm{~s}, 6 \mathrm{H}) .{ }^{13} \mathrm{C}-\mathrm{NMR}\left(100 \mathrm{MHz}, \mathrm{CD}_{3} \mathrm{CO}_{2} \mathrm{D}\right)$ (two carbon resonances are missing due to overlap): $\delta=172.9\left(\mathrm{C}_{\mathrm{q}}\right), 171.9\left(\mathrm{C}_{\mathrm{q}}\right), 170.3\left(\mathrm{C}_{\mathrm{q}}\right), 140.2$ $\left(\mathrm{C}_{\mathrm{q}}\right), 138.5\left(\mathrm{C}_{\mathrm{q}}\right), 138.1\left(\mathrm{C}_{\mathrm{q}}\right), 138.1\left(\mathrm{C}_{\mathrm{q}}\right), 134.6\left(\mathrm{C}_{\mathrm{q}}\right), 133.7\left(\mathrm{C}_{\mathrm{q}}\right), 132.2(\mathrm{CH}), 132.0(\mathrm{CH}), 128.9$ $(\mathrm{CH}), 128.6(\mathrm{CH}), 128.1(\mathrm{CH}), 128.0(\mathrm{CH}), 126.4(\mathrm{CH}), 125.8(\mathrm{CH}), 124.6(\mathrm{CH}), 124.5(\mathrm{CH}), 72.6$ $(\mathrm{CH}), 71.7(\mathrm{CH}), 69.6(\mathrm{CH}), 20.0\left(\mathrm{CH}_{3}\right), 20.0\left(\mathrm{CH}_{3}\right)$. IR (ATR): 3030, 2920, 1651, 1602, 1455, $1390,1345,858,753,698,664 \mathrm{~cm}^{-1} \cdot[\alpha]_{\mathrm{D}}^{20}:-97.7\left(\mathrm{c}=1.00, \mathrm{CHCl}_{3}\right)$. MS (ESI-TOF) $\mathrm{m} / z$ (relative intensity): $1087(10)[2 \mathrm{M}+\mathrm{Na}]^{+}, 533(100)[\mathrm{M}+\mathrm{H}]^{+}$. HR-MS (ESI-TOF) $m / z:[\mathrm{M}+\mathrm{H}]^{+}$Calcd. for $\mathrm{C}_{34} \mathrm{H}_{33} \mathrm{~N}_{2} \mathrm{O}_{4}$ 533.2435; Found 533.2436. 
<smiles>Cc1ccc(C(=O)N2C(C(=O)O)N(C(=O)c3ccc(C)cc3)[C@H](c3ccccc3)[C@H]2c2ccccc2)cc1</smiles>

(4S,5S)-1,3-Bis(4-methylbenzoyl)-4,5-diphenylimidazolidine-2-carboxylic acid (CA8):

General procedure for the preparation of chiral acid was followed using 4-methylbenzoyl chloride (323.0 $\mathrm{mg}, 2.10 \mathrm{mmol})$ to afford $(4 S, 5 S)$-1,3-dibenzoyl-4,5-diphenylimidazolidine-2-carboxylic acid CA8 (352.0 mg, 70\% yield) as a white solid. M.p.: $168-175{ }^{\circ} \mathrm{C} .{ }^{1} \mathrm{H}-\mathrm{NMR}(400 \mathrm{MHz}$, $\left.\mathrm{CD}_{3} \mathrm{CO}_{2} \mathrm{D}\right): \delta=7.49$ (d, $\left.J=7.4 \mathrm{~Hz}, 2 \mathrm{H}\right), 7.45-7.40(\mathrm{~m}, 2 \mathrm{H}), 7.39-7.35(\mathrm{~m}, 1 \mathrm{H}), 7.35-7.21(\mathrm{~m}$, 7H), 7.21-7.12 (m, 2H), 7.13-6.91 (m, 5H), 5.59 (s, 1H), 5.32 (bs, 1H), 2.30 (s, 3H), 2.27 (s, 3H). ${ }^{13} \mathrm{C}-\mathrm{NMR}\left(100 \mathrm{MHz}, \mathrm{CD}_{3} \mathrm{CO}_{2} \mathrm{D}\right): \delta=173.8\left(\mathrm{C}_{\mathrm{q}}\right), 173.0\left(\mathrm{C}_{\mathrm{q}}\right), 171.4\left(\mathrm{C}_{\mathrm{q}}\right), 142.8\left(\mathrm{C}_{\mathrm{q}}\right), 142.4\left(\mathrm{C}_{\mathrm{q}}\right)$, $141.4\left(\mathrm{C}_{\mathrm{q}}\right), 139.3\left(\mathrm{C}_{\mathrm{q}}\right), 132.9\left(\mathrm{C}_{\mathrm{q}}\right), 131.8\left(\mathrm{C}_{\mathrm{q}}\right), 130.1(\mathrm{CH}), 130.0(\mathrm{CH}), 129.9(\mathrm{CH}), 129.9(\mathrm{CH})$, $129.3(\mathrm{CH}), 129.2(\mathrm{CH}), 128.2(\mathrm{CH}), 128.1(\mathrm{CH}), 127.3(\mathrm{CH}), 126.7(\mathrm{CH}), 73.4(\mathrm{CH}), 72.7(\mathrm{CH})$, $70.9(\mathrm{CH}), 21.4\left(\mathrm{CH}_{3}\right), 21.4\left(\mathrm{CH}_{3}\right)$. IR (ATR): 3061, 3008, 1635, 1455, 1377, 1388, 1211, 1182 , $828,752,697 \mathrm{~cm}^{-1} \cdot[\alpha]_{\mathrm{D}}^{20}:-57.4\left(\mathrm{c}=1.00, \mathrm{CHCl}_{3}\right)$. MS (ESI-TOF) $\mathrm{m} / z$ (relative intensity): 1031 (10) $[2 \mathrm{M}+\mathrm{Na}]^{+}, 527(100)[\mathrm{M}+\mathrm{Na}]^{+}, 505(20)[\mathrm{M}+\mathrm{H}]^{+}$. HR-MS (ESI-TOF) $m / z:[\mathrm{M}+\mathrm{Na}]^{+}$ Calcd. for $\mathrm{C}_{32} \mathrm{H}_{28} \mathrm{~N}_{2} \mathrm{O}_{4} \mathrm{Na}$ 527.1941; Found 527.1929.<smiles>CCc1ccc(C(=O)N2C(C(=O)O)N(C(=O)c3ccc(CC)cc3)[C@H](c3ccccc3)[C@H]2c2ccccc2)cc1</smiles>

(4S,5S)-1,3-Bis(4-ethylbenzoyl)-4,5-diphenylimidazolidine-2-carboxylic acid (CA9): General procedure for the preparation of chiral acid was followed using 4-ethylbenzoyl chloride (352.8 $\mathrm{mg}$, $2.10 \mathrm{mmol}$ ) to afford CA9 (361.0 mg, 68\% yield) as a white solid. M.p.: >200 ${ }^{\circ} \mathrm{C} .{ }^{1} \mathrm{H}-\mathrm{NMR}(400$ $\left.\mathrm{MHz}, \mathrm{CD}_{3} \mathrm{CO}_{2} \mathrm{D}\right): \delta=7.50-7.34(\mathrm{~m}, 5 \mathrm{H}), 7.34-7.22(\mathrm{~m}, 7 \mathrm{H}), 7.21-6.92(\mathrm{~m}, 7 \mathrm{H}), 5.57(\mathrm{~s}, 1 \mathrm{H})$, $5.36(\mathrm{bs}, 1 \mathrm{H}), 2.60(\mathrm{p}, J=7.5 \mathrm{~Hz}, 4 \mathrm{H}), 1.17(\mathrm{t}, J=7.5 \mathrm{~Hz}, 6 \mathrm{H}) .{ }^{13} \mathrm{C}-\mathrm{NMR}\left(100 \mathrm{MHz}, \mathrm{CD}_{3} \mathrm{CO}_{2} \mathrm{D}\right)$ (one carbon resonance is missing due to overlap): $\delta=173.7\left(\mathrm{C}_{\mathrm{q}}\right), 173.0\left(\mathrm{C}_{\mathrm{q}}\right), 171.4\left(\mathrm{C}_{\mathrm{q}}\right), 148.9$ $\left(\mathrm{C}_{\mathrm{q}}\right), 148.5\left(\mathrm{C}_{\mathrm{q}}\right), 141.3\left(\mathrm{C}_{\mathrm{q}}\right), 139.3\left(\mathrm{C}_{\mathrm{q}}\right), 133.2\left(\mathrm{C}_{\mathrm{q}}\right), 132.1\left(\mathrm{C}_{\mathrm{q}}\right), 130.1(\mathrm{CH}), 129.8(\mathrm{CH}), 129.3$ 
$(\mathrm{CH}), 129.2(\mathrm{CH}), 128.8(\mathrm{CH}), 128.7(\mathrm{CH}), 128.3(\mathrm{CH}), 127.4(\mathrm{CH}), 126.8(\mathrm{CH}), 73.5(\mathrm{CH}), 72.8$ (CH), $70.9(\mathrm{CH}), 29.4\left(\mathrm{CH}_{2}\right), 29.4\left(\mathrm{CH}_{2}\right), 15.6\left(\mathrm{CH}_{3}\right), 15.5\left(\mathrm{CH}_{3}\right)$. IR (ATR): 2965, 1637, 1602, $1385,1344,838,867,754,697 \mathrm{~cm}^{-1} \cdot[\alpha]_{\mathrm{D}}^{20}:-57.0\left(\mathrm{c}=1.00, \mathrm{CHCl}_{3}\right)$. MS (ESI-TOF) $\mathrm{m} / z$ (relative intensity): $1087(10)[2 \mathrm{M}+\mathrm{Na}]^{+}, 555(100)\left[\mathrm{M}+\mathrm{Na}^{+}, 533(20)[\mathrm{M}+\mathrm{H}]^{+}\right.$. HR-MS (ESI-TOF) $m / z:[\mathrm{M}+\mathrm{Na}]^{+}$Calcd. for $\mathrm{C}_{34} \mathrm{H}_{32} \mathrm{~N}_{2} \mathrm{O}_{4} \mathrm{Na}$ 555.2254; Found 555.2238.

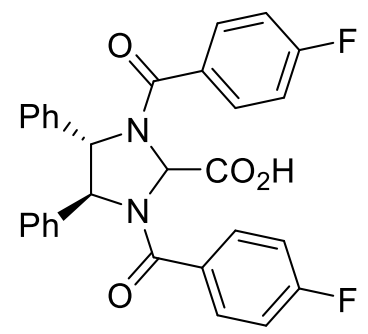

(4S,5S)-1,3-Bis(4-fluorobenzoyl)-4,5-diphenylimidazolidine-2-carboxylic acid (CA10):

General procedure for the preparation of chiral acid was followed using 4-fluorobenzoyl chloride (331.8 mg, $2.10 \mathrm{mmol}$ ) to afford CA10 (312.0 mg, 61\% yield) as a white solid. M.p.: > $200{ }^{\circ} \mathrm{C} .{ }^{1} \mathrm{H}-$ NMR (400 MHz, $\left.\mathrm{CD}_{3} \mathrm{CO}_{2} \mathrm{D}\right): \delta=7.48-7.33(\mathrm{~m}, 9 \mathrm{H}), 7.31-7.24(\mathrm{~m}, 3 \mathrm{H}), 7.17-7.08(\mathrm{~m}, 2 \mathrm{H}), 7.06-$ $6.89(\mathrm{~m}, 5 \mathrm{H}), 5.49(\mathrm{~d}, J=3.5 \mathrm{~Hz}, 1 \mathrm{H}), 5.28(\mathrm{bs}, 1 \mathrm{H}) .{ }^{13} \mathrm{C}-\mathrm{NMR}\left(100 \mathrm{MHz}, \mathrm{CD}_{3} \mathrm{CO}_{2} \mathrm{D}\right)$ (two carbon resonances are missing due to overlap): $\delta=172.5\left(\mathrm{C}_{\mathrm{q}}\right), 171.8\left(\mathrm{C}_{\mathrm{q}}\right), 171.8\left(\mathrm{C}_{\mathrm{q}}\right), 164.8(\mathrm{~d}$, $\left.{ }^{1} J_{\mathrm{C}-\mathrm{F}}=251.5 \mathrm{~Hz}, \mathrm{C}_{\mathrm{q}}\right), 164.7\left(\mathrm{~d},{ }^{1} J_{\mathrm{C}-\mathrm{F}}=251.5 \mathrm{~Hz}, \mathrm{C}_{\mathrm{q}}\right), 140.8\left(\mathrm{C}_{\mathrm{q}}\right), 139.1\left(\mathrm{C}_{\mathrm{q}}\right), 132.2\left(\mathrm{~d},{ }^{4} J_{\mathrm{C}-\mathrm{F}}=3.2\right.$ $\left.\mathrm{Hz}, \mathrm{C}_{\mathrm{q}}\right), 131.2\left(\mathrm{~d},{ }^{4} J_{\mathrm{C}-\mathrm{F}}=3.4 \mathrm{~Hz}, \mathrm{C}_{\mathrm{q}}\right), 130.6\left(\mathrm{~d},{ }^{3} J_{\mathrm{C}-\mathrm{F}}=8.3 \mathrm{~Hz}, \mathrm{CH}\right), 130.1(\mathrm{CH}), 129.9(\mathrm{CH}), 129.3$ $\left(\mathrm{d},{ }^{3} J_{\mathrm{C}-\mathrm{F}}=7.0 \mathrm{~Hz}, \mathrm{CH}\right), 127.5(\mathrm{CH}), 127.0(\mathrm{CH}), 116.4\left(\mathrm{~d},{ }^{2} J_{\mathrm{C}-\mathrm{F}}=22.1 \mathrm{~Hz}, \mathrm{CH}\right), 116.3\left(\mathrm{~d},{ }^{2} J_{\mathrm{C}-\mathrm{F}}=\right.$ $22.1 \mathrm{~Hz}, \mathrm{CH}), 74.3(\mathrm{CH}), 73.1(\mathrm{CH}), 71.3(\mathrm{CH}) .{ }^{19} \mathrm{~F}-\mathrm{NMR}\left(377 \mathrm{MHz}, \mathrm{CD}_{3} \mathrm{CO}_{2} \mathrm{D}\right): \delta=-109.3(\mathrm{~m})$,

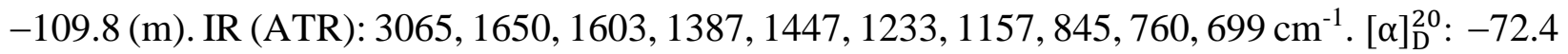
$\left(\mathrm{c}=1.00, \mathrm{CHCl}_{3}\right)$. MS (ESI-TOF) $\mathrm{m} / \mathrm{z}$ (relative intensity): $1047(10)[2 \mathrm{M}+\mathrm{Na}]^{+}, 535(100)[\mathrm{M}+$ $\mathrm{Na}]^{+}, 513(15)[\mathrm{M}+\mathrm{H}]^{+}$. HR-MS (ESI-TOF) $m / z:[\mathrm{M}+\mathrm{Na}]^{+}$Calcd. for $\mathrm{C}_{30} \mathrm{H}_{22}{ }^{19} \mathrm{~F}_{2} \mathrm{~N}_{2} \mathrm{O}_{4} \mathrm{Na}$ 535.1440 found 535.1426 . 


\section{Optimization Studies}

Scheme S1. Ligand Screening for the Enantioselective C-H Alkylation.

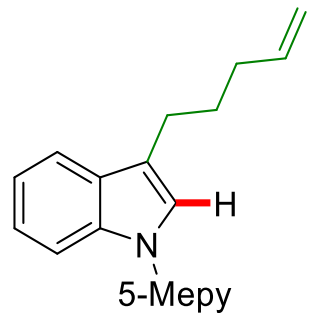

$1 \mathrm{a}$<smiles>CCC(C)[C@H](NC(=O)OCc1ccccc1)C(=O)O</smiles>

CA1: $90 \%, 44: 56$ e.r.<smiles>O=C(O)C1N(C(=O)c2ccccc2)[C@@H](c2ccccc2)[C@H](c2ccccc2)N1C(=O)c1ccccc1</smiles>

$\left[\operatorname{RuCl}_{2}(p \text {-cymene })\right]_{2}(5.0 \mathrm{~mol} \%)$ $\mathrm{AgSbF}_{6}(20 \mathrm{~mol} \%)$

CA $(20 \mathrm{~mol} \%)$

PhMe, RT, 12 h

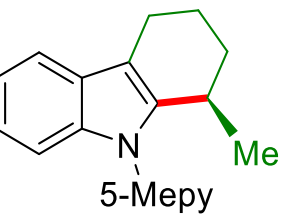

$2 a$<smiles>O=P1(O)Oc2ccc3ccccc3c2-c2c(ccc3ccccc23)O1</smiles>

CA2: $88 \%, 59: 41$ e.r.

CA3: ---
$\mathrm{Ar}=\mathrm{C}_{6} \mathrm{H}_{6}$ (CA4): $\quad 92 \%, 84: 16$ e.r.

$\mathrm{Ar}=3-\mathrm{Me}-\mathrm{C}_{6} \mathrm{H}_{4}$ (CA5): $\quad 90 \%, 76: 24$ e.r.

$\mathrm{Ar}=3-\mathrm{CF}_{3}-\mathrm{C}_{6} \mathrm{H}_{4}(\mathrm{CA} 6): \quad 88 \%, 82: 18$ e.r.

$\mathrm{Ar}=3,5-\mathrm{Me}-\mathrm{C}_{6} \mathrm{H}_{3}$ (CA7) 93\%, 78:22 e.r.

$\mathrm{Ar}=4-\mathrm{Me}-\mathrm{C}_{6} \mathrm{H}_{4}$ (CA8): $\quad 95 \%, 85: 15$ e.r.

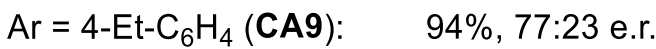

$\mathrm{Ar}=4-\mathrm{F}-\mathrm{C}_{6} \mathrm{H}_{4}(\mathrm{CA10}): \quad 90 \% 72: 28$ e.r.

Table S1. Asymmetric Ruthenium(II)-Catalyzed C-H Activation. ${ }^{a}$

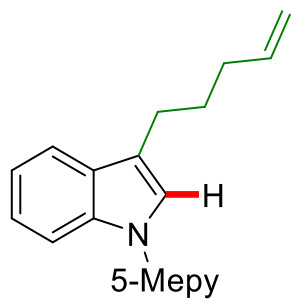

$1 \mathbf{a}$
$\left[\mathrm{RuCl}_{2}(p \text {-cymene })\right]_{2}(5.0 \mathrm{~mol} \%)$

CA8 $(20 \mathrm{~mol} \%)$

$\mathrm{AgSbF}_{6}(20 \mathrm{~mol} \%)$

PhMe, RT, $12 \mathrm{~h}$

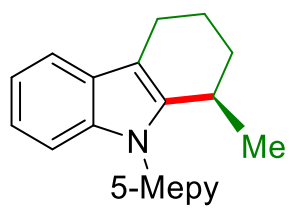

$2 a$

\begin{tabular}{cccc}
\hline entry & variation of standard condition & yield [\%] & e.r. \\
\hline 1 & none & 95 & $85: 15$ \\
2 & $\mathrm{NaSbF}_{6}$ instead of $\mathrm{AgSbF}_{6}$ & $37^{b}$ & $77: 23$
\end{tabular}




$\begin{array}{cccc}3 & \mathrm{NaPF}_{6} \text { instead of } \mathrm{AgSbF}_{6} & 20^{b} & 83: 17 \\ 4 & \text { Amberlyst } 15 \text { as additive } & 94 & 85: 15 \\ 5 & \mathrm{DCE} \text { as solvent instead of } \mathrm{PhMe} & 96 & 79: 21 \\ 6 & {\left[\mathrm{OsCl}_{2}(p \text {-cymene })\right]_{2} \text { as catalyst }} & --- & --- \\ 7 & {\left[\mathrm{Cp}^{*} \mathrm{Co}(\mathrm{CO}) \mathrm{I}_{2}\right] \text { as catalyst }} & <5 & \text { nd } \\ 8 & \mathrm{Pd}(\mathrm{OAc})_{2} \text { as catalyst } & --- & --- \\ 9 & {\left[\mathrm{Cp} * \mathrm{IrCl}_{2}\right]_{2} \text { as catalyst }} & --- & --- \\ 10 & {\left[\mathrm{Cp} * \mathrm{RhCl}_{2}\right]_{2} \text { as catalyst }} & 90 & 58: 42 \\ 11 & \text { Without catalyst } & --- & --- \\ 12 & \text { Without } \mathrm{AgSbF}_{6} & --- & ---\end{array}$

${ }^{a}$ General reaction conditions: 1a $(0.25 \mathrm{mmol}),[\mathrm{Ru}](10 \mathrm{~mol} \%), \operatorname{AgSbF}_{6}(20 \mathrm{~mol} \%)$, CA8 $(20 \mathrm{~mol} \%)$, PhMe $(0.50 \mathrm{~mL}), \mathrm{RT}, 12 \mathrm{~h}$, isolated yields. Enantioselectivities determined by chiral HPLC. ${ }^{b}$ conversion determined by ${ }^{1} \mathrm{H}-\mathrm{NMR}$ using $\mathrm{Ph}_{3} \mathrm{CH}$ as the internal standard. nd $=$ not determined.

Table S2. Asymmetric Ruthenium(II)-Catalyzed C-H Activation for the synthesis of cyclohepta[b]indoles. ${ }^{a}$

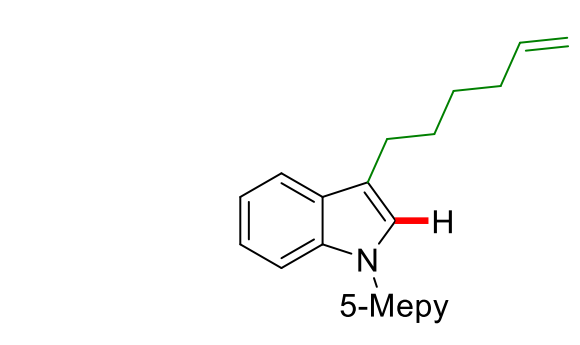

\begin{tabular}{ll} 
& $3 a$ \\
\hline entry & variation $c$
\end{tabular}

$\left[\mathrm{RuCl}_{2}(p \text {-cymene })\right]_{2}(5.0 \mathrm{~mol} \%)$

CA8 $(20 \mathrm{~mol} \%)$

$\mathrm{AgSbF}_{6}(20 \mathrm{~mol} \%)$

PhMe, RT, $20 \mathrm{~h}$

\begin{tabular}{cccc}
\hline 1 & none & 17 & $82: 18$ \\
2 & Amberlyst 15 as additive & $25^{b}$ & $82: 18$ \\
3 & Amberlyst 15 as additive and $72 \mathrm{~h}$ instead of $20 \mathrm{~h}$ & $51^{b}$ & $82: 18$ \\
\hline
\end{tabular}

${ }^{a}$ General reaction conditions: 3a $(0.10 \mathrm{mmol}),[\mathrm{Ru}](10 \mathrm{~mol} \%), \operatorname{AgSbF}_{6}(20 \mathrm{~mol} \%), \mathbf{C A 8}(20 \mathrm{~mol} \%)$, $\mathrm{PhMe}(0.50 \mathrm{~mL}), \mathrm{RT}, 20 \mathrm{~h}$, isolated yields. ${ }^{b} 1.5$ equiv of Amberlyst 15 was used. 
General Procedure A: Ruthenium(II)-Catalyzed Asymmetric C-H Alkylation for the synthesis of Tetrahydrocarbazoles

A suspension of indole 1 (0.25 mmol, 1.00 equiv), [ $\mathrm{RuCl}_{2}(p$-cymene $\left.)\right]_{2}(7.7 \mathrm{mg}, 5.0 \mathrm{~mol} \%)$, $\operatorname{AgSbF}_{6}(17.2 \mathrm{mg}, 20 \mathrm{~mol} \%)$, chiral acid CA8 $(25.2 \mathrm{mg}, 20 \mathrm{~mol} \%)$ in PhMe $(0.50 \mathrm{~mL})$ were stirred at $25{ }^{\circ} \mathrm{C}$ for $12 \mathrm{~h}$. The reaction mixture was diluted with EtOAc $(2.0 \mathrm{~mL})$ and the solvent was removed in vacuo. The crude mixture was purified by column chromatography on silica gel to afford the desired product 2.

General Procedure B: Ruthenium(II)-Catalyzed Asymmetric C-H Alkylation for the synthesis of cyclohepta $[b]$ indoles

A suspension of indole $3\left(0.10 \mathrm{mmol}, 1.00 \text { equiv), [ } \mathrm{RuCl}_{2}(p \text {-cymene })\right]_{2}(3.1 \mathrm{mg}, 5.0 \mathrm{~mol} \%)$, $\mathrm{AgSbF}_{6}$ (6.9 mg, $20 \mathrm{~mol} \%$ ), chiral acid CA8 (10.1 mg, $20 \mathrm{~mol} \%$ ) and Amberlyst 15 (32 mg, 1.5 equiv) in $\mathrm{PhMe}(0.50 \mathrm{~mL})$ were stirred at $25^{\circ} \mathrm{C}$ for $72 \mathrm{~h}$. The reaction mixture was diluted with EtOAc $(2.0 \mathrm{~mL})$ and the solvent was removed in vacuo. The crude mixture was purified by column chromatography on silica gel to afford the desired product 4.

\section{General Procedure for the Synthesis of Racemic Products}

A suspension of indole 1 or 3 (0.10 mmol, 1.00 equiv), [ $\mathrm{RuCl}_{2}(p$-cymene $\left.)\right]_{2}(3.1 \mathrm{mg}, 5.0 \mathrm{~mol} \%)$, $\mathrm{AgSbF}_{6}(6.9 \mathrm{mg}, 100 \mu \mathrm{mol}, 20 \mathrm{~mol} \%), 1-\mathrm{AdCO}_{2} \mathrm{H}(18.0 \mathrm{mg}, 1$ equiv) in $\mathrm{PhMe}(0.50 \mathrm{~mL})$ were stirred at $50{ }^{\circ} \mathrm{C}$ for $12 \mathrm{~h}$. At ambient temperature, the reaction mixture was diluted with EtOAc $(2.0 \mathrm{~mL})$ and the solvents were removed in vacuo. The crude mixture was purified by column chromatography on silica gel to afford the racemic products $r a c-2$ or $\mathbf{4}$. 


\section{Synthesis of Starting Materials 1 and 3}

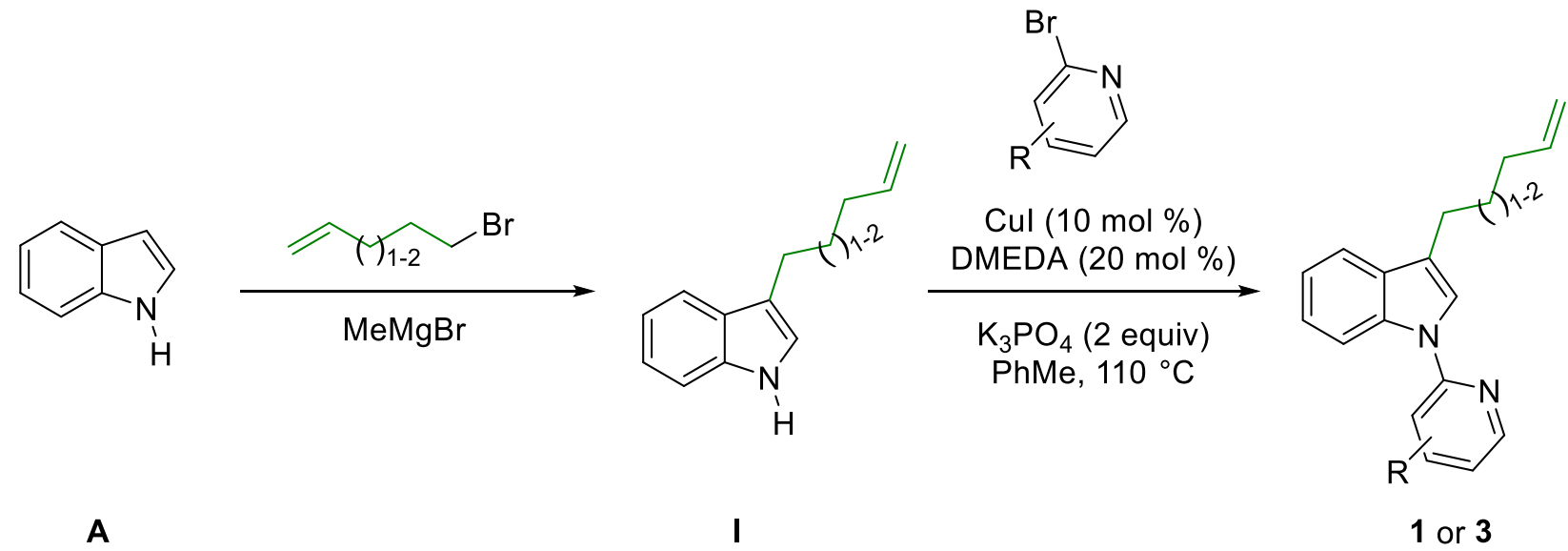

The procedure for the alkylation of indoles was adapted from a previous literature report. ${ }^{4 a}$ To a solution of $\mathrm{MeMgBr}\left(5.0 \mathrm{mmol}, 3.0 \mathrm{M}\right.$ solution in $\left.\mathrm{Et}_{2} \mathrm{O}\right)$ in $\mathrm{PhMe}(10 \mathrm{ml})$ was added a solution of indole $\mathbf{A}(4.5 \mathrm{mmol})$ at $\mathrm{rt}$. After $10 \mathrm{~min}, 5$-bromopentene or 6-bromohexene (3 $\mathrm{mmol})$ was added and the reaction mixture was allowed to stir at $80{ }^{\circ} \mathrm{C}$. After $24 \mathrm{~h}$, the reaction mixture was cooled and quenched with sat. $\mathrm{NH}_{4} \mathrm{Cl}$. The organic phase was separated and the aqueous phase was extracted with EtOAc twice. The organic layer was washed with water and brine, dried over $\mathrm{Na}_{2} \mathrm{SO}_{4}$, and concentrated in vacuo. The residue was purified by short silica filtration by eluting EtOAc-hexane to give $\mathbf{I}$ as a colorless oil. Next, $N$-pyridyl indoles were synthesized following a previous literature report. ${ }^{4 \mathrm{~b}} \mathrm{~A}$ mixture of indole $\mathbf{I}$, 2-bromopyridine (3 mmol), CuI (10 mol \%), $N$, $N$ '-dimethyl-ethylenediamine (20 mol \%) and $\mathrm{K}_{3} \mathrm{PO}_{4}(6 \mathrm{mmol})$ in $\mathrm{PhMe}(15 \mathrm{~mL})$ was vigorously stirred at $110{ }^{\circ} \mathrm{C}$ under nitrogen atmosphere for $12 \mathrm{~h}$. After cooling the mixture to ambient temperature, the reaction mixture was diluted with EtOAc $(20 \mathrm{~mL})$ and washed with $\mathrm{H}_{2} \mathrm{O}(2 \times 10$ $\mathrm{mL})$. The aqueous phase was extracted with EtOAc $(2 \times 10 \mathrm{~mL})$, and the combined organic phase was dried over $\mathrm{Na}_{2} \mathrm{SO}_{4}$. After filtration and evaporation of the solvents in vacuo, the crude product was purified by column chromatography on silica gel to give $\mathbf{1}$ or $\mathbf{3}$. 


\section{Characterization Data of Starting Materials 1 and 3}

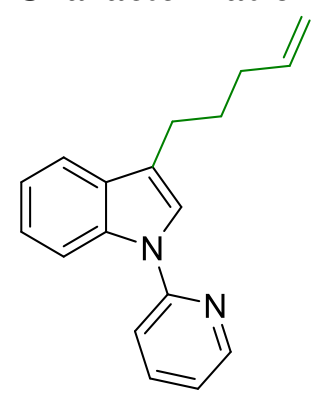

3-(Pent-4-en-1-yl)-1-(pyridin-2-yl)-1H-indole (1a'): Yellow oil (408 mg, 52\% yield). ${ }^{1} \mathrm{H}-\mathrm{NMR}$ $\left(400 \mathrm{MHz}, \mathrm{CDCl}_{3}\right): \delta=8.55(\mathrm{~d}, J=4.8 \mathrm{~Hz}, 1 \mathrm{H}), 8.20(\mathrm{~d}, J=8.3 \mathrm{~Hz}, 1 \mathrm{H}), 7.84-7.75(\mathrm{~m}, 1 \mathrm{H}), 7.63$ $(\mathrm{d}, J=7.8 \mathrm{~Hz}, 1 \mathrm{H}), 7.54(\mathrm{~s}, 1 \mathrm{H}), 7.48(\mathrm{~d}, J=8.3 \mathrm{~Hz}, 1 \mathrm{H}), 7.35-7.28(\mathrm{~m}, 1 \mathrm{H}), 7.24-7.18(\mathrm{~m}, 1 \mathrm{H})$, $7.13(\mathrm{dd}, J=7.8,4.9 \mathrm{~Hz}, 1 \mathrm{H}), 5.89(\mathrm{ddt}, J=16.9,9.5,6.6 \mathrm{~Hz}, 1 \mathrm{H}), 5.15-4.92(\mathrm{~m}, 2 \mathrm{H}), 2.82(\mathrm{t}, J$ $=7.6 \mathrm{~Hz}, 2 \mathrm{H}), 2.21(\mathrm{q}, J=7.3 \mathrm{~Hz}, 2 \mathrm{H}), 1.88(\mathrm{p}, J=7.6 \mathrm{~Hz}, 2 \mathrm{H}) .{ }^{13} \mathrm{C}-\mathrm{NMR}\left(100 \mathrm{MHz}, \mathrm{CDCl}_{3}\right): \delta$ $=152.7\left(\mathrm{C}_{\mathrm{q}}\right), 149.1(\mathrm{CH}), 138.8(\mathrm{CH}), 138.4(\mathrm{CH}), 135.6\left(\mathrm{C}_{\mathrm{q}}\right), 130.5\left(\mathrm{C}_{\mathrm{q}}\right), 123.3(\mathrm{CH}), 123.0(\mathrm{CH})$, $120.9(\mathrm{CH}), 119.7\left(\mathrm{C}_{\mathrm{q}}\right), 119.6(\mathrm{CH}), 119.3(\mathrm{CH}), 114.9\left(\mathrm{CH}_{2}\right), 114.3(\mathrm{CH}), 113.2(\mathrm{CH}), 33.8$ $\left(\mathrm{CH}_{2}\right), 29.0\left(\mathrm{CH}_{2}\right), 24.6\left(\mathrm{CH}_{2}\right)$. IR (ATR): 2927, 2855, 1590, 1470, 1452, 1368, $732 \mathrm{~cm}^{-1} . \mathrm{MS}$ (ESI-TOF) $m / z$ (relative intensity): $285(5)[\mathrm{M}+\mathrm{Na}]^{+}, 263(100)[\mathrm{M}+\mathrm{H}]^{+}$. HR-MS (ESI-TOF) $m / z:[\mathrm{M}+\mathrm{H}]^{+}$Calcd. for $\mathrm{C}_{18} \mathrm{H}_{19} \mathrm{~N}_{2} 263.1543$; Found 263.1549 .

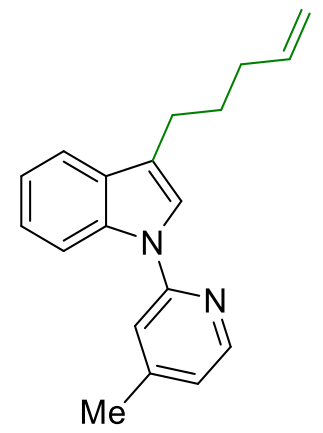

1-(4-Methylpyridin-2-yl)-3-(pent-4-en-1-yl)-1H-indole (1a'): Yellow oil (372 mg, 45\% yield). ${ }^{1} \mathrm{H}-\mathrm{NMR}\left(400 \mathrm{MHz}, \mathrm{CDCl}_{3}\right): \delta=8.40(\mathrm{~d}, J=5.1 \mathrm{~Hz}, 1 \mathrm{H}), 8.19(\mathrm{~d}, J=8.3 \mathrm{~Hz}, 1 \mathrm{H}), 7.62(\mathrm{~d}, J=$ $7.8 \mathrm{~Hz}, 1 \mathrm{H}), 7.52(\mathrm{~s}, 1 \mathrm{H}), 7.34-7.26(\mathrm{~m}, 2 \mathrm{H}), 7.23-7.15(\mathrm{~m}, 1 \mathrm{H}), 6.96(\mathrm{~d}, J=5.1 \mathrm{~Hz}, 1 \mathrm{H}), 5.89$ (ddt, $J=16.9,10.1,6.6 \mathrm{~Hz}, 1 \mathrm{H}), 5.15-4.95(\mathrm{~m}, 2 \mathrm{H}), 2.81(\mathrm{t}, J=7.7 \mathrm{~Hz}, 2 \mathrm{H}), 2.44(\mathrm{~s}, 3 \mathrm{H}), 2.20$ (q, $J=7.2 \mathrm{~Hz}, 2 \mathrm{H}), 1.87(\mathrm{p}, J=7.6 \mathrm{~Hz}, 2 \mathrm{H}) .{ }^{13} \mathrm{C}-\mathrm{NMR}\left(100 \mathrm{MHz}, \mathrm{CDCl}_{3}\right): \delta=152.9\left(\mathrm{C}_{\mathrm{q}}\right), 149.7$ $\left(\mathrm{C}_{\mathrm{q}}\right), 148.7(\mathrm{CH}), 138.9(\mathrm{CH}), 135.6\left(\mathrm{C}_{\mathrm{q}}\right), 130.5\left(\mathrm{C}_{\mathrm{q}}\right), 123.2(\mathrm{CH}), 123.1(\mathrm{CH}), 121.0(\mathrm{CH}), 120.8$ $(\mathrm{CH}), 119.4\left(\mathrm{C}_{\mathrm{q}}\right), 119.3(\mathrm{CH}), 115.0(\mathrm{CH}), 114.8\left(\mathrm{CH}_{2}\right), 113.2(\mathrm{CH}), 33.8\left(\mathrm{CH}_{2}\right), 29.1\left(\mathrm{CH}_{2}\right), 24.6$ $\left(\mathrm{CH}_{2}\right), 21.5\left(\mathrm{CH}_{3}\right)$. IR (ATR): 2927, 1602, 1560, 1452, 1424, 1200, $737 \mathrm{~cm}^{-1}$. MS (ESI-TOF) $\mathrm{m} / z$ 
(relative intensity): $293(5)[\mathrm{M}+\mathrm{Na}]^{+}, 277(100)[\mathrm{M}+\mathrm{H}]^{+}$. HR-MS (ESI-TOF) $m / z:[\mathrm{M}+\mathrm{H}]^{+}$ Calcd. for $\mathrm{C}_{19} \mathrm{H}_{21} \mathrm{~N}_{2}$ 277.1699; Found 277.1708.

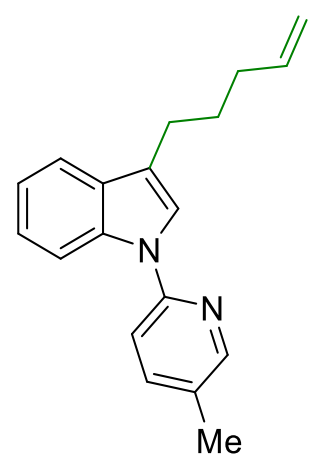

1-(5-Methylpyridin-2-yl)-3-(pent-4-en-1-yl)-1H-indole (1a): Yellow oil (390 mg, 47\% yield). ${ }^{1} \mathrm{H}$ NMR $\left(400 \mathrm{MHz}, \mathrm{CDCl}_{3}\right): \delta=8.37(\mathrm{~d}, J=3.6 \mathrm{~Hz}, 1 \mathrm{H}), 8.14(\mathrm{~d}, J=8.2 \mathrm{~Hz}, 1 \mathrm{H}), 7.64(\mathrm{~d}, J=$ $8.0 \mathrm{~Hz}, 1 \mathrm{H}), 7.61(\mathrm{~d}, J=8.4 \mathrm{~Hz}, 1 \mathrm{H}), 7.51(\mathrm{~s}, 1 \mathrm{H}), 7.38(\mathrm{~d}, J=8.3 \mathrm{~Hz}, 1 \mathrm{H}), 7.29$ (t, $J=7.7 \mathrm{~Hz}$, $1 \mathrm{H}), 7.21(\mathrm{t}, J=7.4 \mathrm{~Hz}, 1 \mathrm{H}), 5.90(\mathrm{ddt}, J=16.8,9.9,6.6 \mathrm{~Hz}, 1 \mathrm{H}), 5.15-4.93(\mathrm{~m}, 2 \mathrm{H}), 2.83(\mathrm{t}, J=$ $7.6 \mathrm{~Hz}, 2 \mathrm{H}), 2.38(\mathrm{~s}, 3 \mathrm{H}), 2.21$ (q, $J=7.3 \mathrm{~Hz}, 2 \mathrm{H}), 1.89$ (p, $J=7.6 \mathrm{~Hz}, 2 \mathrm{H}) .{ }^{13} \mathrm{C}-\mathrm{NMR}(100 \mathrm{MHz}$, $\left.\mathrm{CDCl}_{3}\right): \delta=150.6\left(\mathrm{C}_{\mathrm{q}}\right), 149.1(\mathrm{CH}), 139.0(\mathrm{CH}), 138.9(\mathrm{CH}), 135.5\left(\mathrm{C}_{\mathrm{q}}\right), 130.3\left(\mathrm{C}_{\mathrm{q}}\right), 129.2\left(\mathrm{C}_{\mathrm{q}}\right)$, $123.2(\mathrm{CH}), 123.1(\mathrm{CH}), 120.6(\mathrm{CH}), 119.3\left(\mathrm{C}_{\mathrm{q}}\right), 119.2(\mathrm{CH}), 114.8\left(\mathrm{CH}_{2}\right), 114.1(\mathrm{CH}), 112.84$ (CH), $33.8\left(\mathrm{CH}_{2}\right), 29.1\left(\mathrm{CH}_{2}\right), 24.6\left(\mathrm{CH}_{2}\right), 17.9\left(\mathrm{CH}_{3}\right)$. IR (ATR): 2922, 2850, 1602, 1487, 1394, 1217, $741 \mathrm{~cm}^{-1}$. MS (ESI-TOF) $\mathrm{m} / z$ (relative intensity): $277(100)[\mathrm{M}+\mathrm{H}]^{+}$. HR-MS (ESI-TOF) $m / z:[\mathrm{M}+\mathrm{H}]^{+}$Calcd. for $\mathrm{C}_{19} \mathrm{H}_{21} \mathrm{~N}_{2} 277.1699$; Found 277.1705.

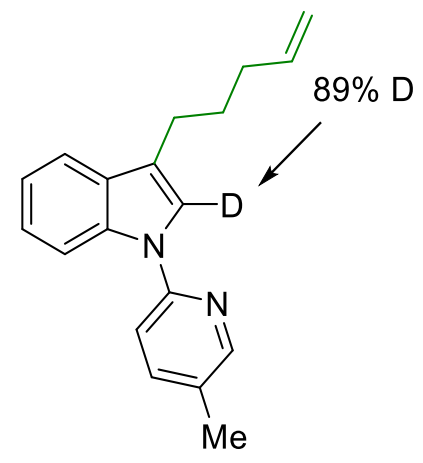

1-(5-Methylpyridin-2-yl)-3-(pent-4-en-1-yl)-1H-indole-2-D ([D]1-1a): 2-Deuterium indole was prepared according to a literature procedure. ${ }^{5}$ Next general procedure for the synthesis of starting material 1 was followed for the synthesis of [D]1-1a. Yellow oil (372 mg, 45\% yield, 89\% D). 


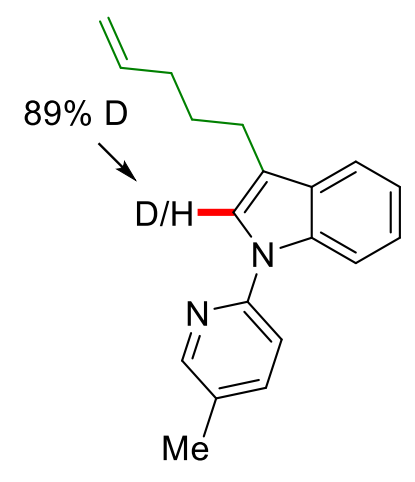

[D] $]_{1}-1 \mathrm{a}$

$\left(300 \mathrm{MHz}, \mathrm{CDCl}_{3}\right)$
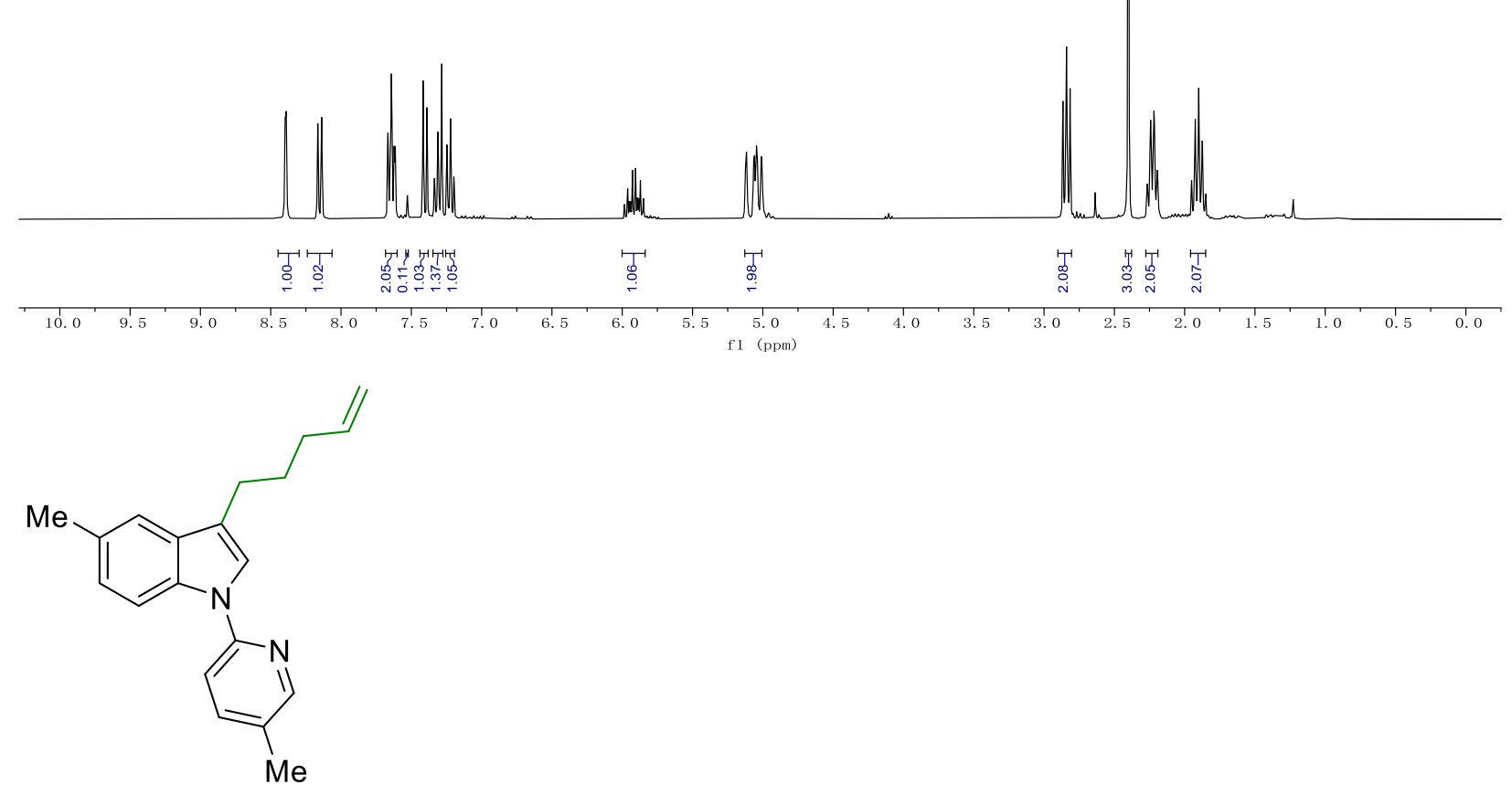

5-Methyl-1-(5-methylpyridin-2-yl)-3-(pent-4-en-1-yl)-1H-indole (1b): Yellow oil (401 mg, 46\%). ${ }^{1} \mathrm{H}-\mathrm{NMR}\left(400 \mathrm{MHz}, \mathrm{CDCl}_{3}\right): \delta=8.39$ (d, $\left.J=2.4 \mathrm{~Hz}, 1 \mathrm{H}\right), 8.09$ (d, $\left.J=8.5 \mathrm{~Hz}, 1 \mathrm{H}\right), 7.60-$ $7.51(\mathrm{~m}, 2 \mathrm{H}), 7.47(\mathrm{~s}, 1 \mathrm{H}), 7.36(\mathrm{~d}, J=8.3 \mathrm{~Hz}, 1 \mathrm{H}), 7.17(\mathrm{dd}, J=8.4,1.7 \mathrm{~Hz}, 1 \mathrm{H}), 5.96(\mathrm{ddt}, J=$ 16.9, 10.2, $6.6 \mathrm{~Hz}, 1 \mathrm{H}), 5.22-5.00(\mathrm{~m}, 2 \mathrm{H}), 2.85$ (t, $J=7.6 \mathrm{~Hz}, 2 \mathrm{H}), 2.56(\mathrm{~s}, 3 \mathrm{H}), 2.37$ (s, 3H), $2.27(\mathrm{q}, J=7.2 \mathrm{~Hz}, 2 \mathrm{H}), 1.93(\mathrm{p}, J=7.5 \mathrm{~Hz}, 2 \mathrm{H}) .{ }^{13} \mathrm{C}-\mathrm{NMR}\left(100 \mathrm{MHz}, \mathrm{CDCl}_{3}\right)$ : One carbon resonance is missing due to overlap: $\delta=150.6\left(\mathrm{C}_{\mathrm{q}}\right), 148.9(\mathrm{CH}), 138.8(\mathrm{CH}), 133.8\left(\mathrm{C}_{\mathrm{q}}\right), 130.5$ $\left(\mathrm{C}_{\mathrm{q}}\right), 129.8\left(\mathrm{C}_{\mathrm{q}}\right), 128.7\left(\mathrm{C}_{\mathrm{q}}\right), 124.5(\mathrm{CH}), 123.1(\mathrm{CH}), 119.0(\mathrm{CH}), 118.7\left(\mathrm{C}_{\mathrm{q}}\right), 114.7\left(\mathrm{CH}_{2}\right), 113.5$ $(\mathrm{CH}), 112.7(\mathrm{CH}), 33.7\left(\mathrm{CH}_{2}\right), 29.0\left(\mathrm{CH}_{2}\right), 24.6\left(\mathrm{CH}_{2}\right), 21.5\left(\mathrm{CH}_{3}\right), 17.8\left(\mathrm{CH}_{3}\right)$. IR (ATR): 2928 , 
1603, 1489, 1450, 1399, 1211, 992, $819 \mathrm{~cm}^{-1}$. MS (ESI-TOF) $\mathrm{m} / \mathrm{z}$ (relative intensity): 291 (100) $[\mathrm{M}+\mathrm{H}]^{+}$. HR-MS (ESI-TOF) $m / z:[\mathrm{M}+\mathrm{H}]^{+}$Calcd. for $\mathrm{C}_{20} \mathrm{H}_{23} \mathrm{~N}_{2}$ 291.1856; Found 291.1867.

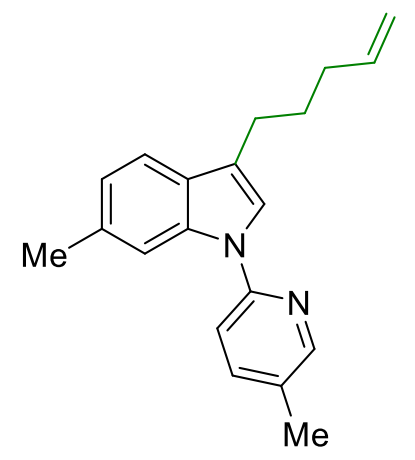

6-Methyl-1-(5-methylpyridin-2-yl)-3-(pent-4-en-1-yl)-1H-indole (1c): Yellow oil (399 mg, 40\%). ${ }^{1} \mathrm{H}-\mathrm{NMR}\left(400 \mathrm{MHz}, \mathrm{CDCl}_{3}\right): \delta=8.37(\mathrm{~d}, J=2.3 \mathrm{~Hz}, 1 \mathrm{H}), 7.93$ (s, 1H), 7.60 (dd, $J=8.4$, $2.3 \mathrm{~Hz}, 1 \mathrm{H}), 7.50(\mathrm{~d}, J=8.0 \mathrm{~Hz}, 1 \mathrm{H}), 7.41(\mathrm{~s}, 1 \mathrm{H}), 7.36(\mathrm{~d}, J=8.3 \mathrm{~Hz}, 1 \mathrm{H}), 7.03(\mathrm{~d}, J=8.0 \mathrm{~Hz}$, 1H), 5.89 (ddt, $J=16.9,10.2,6.6 \mathrm{~Hz}, 1 \mathrm{H}), 5.11-4.94(\mathrm{~m}, 2 \mathrm{H}), 2.79(\mathrm{t}, J=7.6 \mathrm{~Hz}, 2 \mathrm{H}), 2.51$ (s, $3 \mathrm{H}), 2.37(\mathrm{~s}, 3 \mathrm{H}), 2.19$ (q, J=7.2 Hz, 2H), 1.86 (p, $J=7.5 \mathrm{~Hz}, 2 \mathrm{H}) .{ }^{13} \mathrm{C}$ NMR $\left(100 \mathrm{MHz}, \mathrm{CDCl}_{3}\right)$ : $\delta=150.7\left(\mathrm{C}_{\mathrm{q}}\right), 149.0(\mathrm{CH}), 138.9(\mathrm{CH}), 138.9\left(\mathrm{C}_{\mathrm{q}}\right), 135.9\left(\mathrm{C}_{\mathrm{q}}\right), 132.9\left(\mathrm{C}_{\mathrm{q}}\right), 129.0\left(\mathrm{C}_{\mathrm{q}}\right), 128.2\left(\mathrm{C}_{\mathrm{q}}\right)$, 122.6 (CH), $122.2(\mathrm{CH}), 119.1\left(\mathrm{C}_{\mathrm{q}}\right), 118.9(\mathrm{CH}), 114.8\left(\mathrm{CH}_{2}\right), 114.2(\mathrm{CH}), 112.8(\mathrm{CH}), 33.8\left(\mathrm{CH}_{2}\right)$, $29.1\left(\mathrm{CH}_{2}\right), 24.7\left(\mathrm{CH}_{2}\right), 22.2\left(\mathrm{CH}_{3}\right), 17.9\left(\mathrm{CH}_{3}\right)$. IR (ATR): 2922, 2857, 1601, 1493, 1433, 1394, $1195,911,802 \mathrm{~cm}^{-1}$. MS (ESI-TOF) $\mathrm{m} / z$ (relative intensity): $313(5)[\mathrm{M}+\mathrm{Na}]^{+}, 291(100)[\mathrm{M}+$ $\mathrm{H}]^{+}$. HR-MS (ESI-TOF) $m / z:[\mathrm{M}+\mathrm{H}]^{+}$Calcd. for $\mathrm{C}_{20} \mathrm{H}_{23} \mathrm{~N}_{2}$ 291.1856; Found 291.1860.

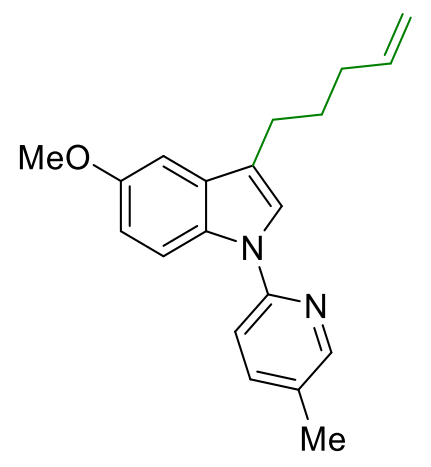

5-Methoxy-1-(5-methylpyridin-2-yl)-3-(pent-4-en-1-yl)-1H-indole (1d): Yellow oil (275 mg, $30 \%$ yield). ${ }^{1} \mathrm{H}-\mathrm{NMR}\left(400 \mathrm{MHz}, \mathrm{CDCl}_{3}\right): \delta=8.34(\mathrm{~d}, J=3.2 \mathrm{~Hz}, 1 \mathrm{H}), 8.08(\mathrm{~d}, J=9.0 \mathrm{~Hz}, 1 \mathrm{H})$, $7.58(\mathrm{dd}, J=8.3,2.4 \mathrm{~Hz}, 1 \mathrm{H}), 7.47(\mathrm{~s}, 1 \mathrm{H}), 7.33(\mathrm{~d}, J=8.3 \mathrm{~Hz}, 1 \mathrm{H}), 7.04$ (d, $J=2.5 \mathrm{~Hz}, 1 \mathrm{H}), 6.92$ $(\mathrm{dd}, J=9.0,2.5 \mathrm{~Hz}, 1 \mathrm{H}), 5.89$ (ddt, $J=16.9,10.1,6.6 \mathrm{~Hz}, 1 \mathrm{H}), 5.23-4.79$ (m, 2H), 3.89 (s, 3H), $2.77(\mathrm{t}, J=7.6 \mathrm{~Hz}, 2 \mathrm{H}), 2.36(\mathrm{~s}, 3 \mathrm{H}), 2.20(\mathrm{q}, J=7.2 \mathrm{~Hz}, 2 \mathrm{H}), 1.86(\mathrm{p}, J=7.5 \mathrm{~Hz}, 2 \mathrm{H}) .{ }^{13} \mathrm{C}-\mathrm{NMR}$ $\left(100 \mathrm{MHz}, \mathrm{CDCl}_{3}\right): \delta=154.7\left(\mathrm{C}_{\mathrm{q}}\right), 150.7\left(\mathrm{C}_{\mathrm{q}}\right), 148.9(\mathrm{CH}), 139.0(\mathrm{CH}), 138.9(\mathrm{CH}), 131.0\left(\mathrm{C}_{\mathrm{q}}\right)$, 
$130.7\left(\mathrm{C}_{\mathrm{q}}\right), 128.8\left(\mathrm{C}_{\mathrm{q}}\right), 123.6(\mathrm{CH}), 118.9\left(\mathrm{C}_{\mathrm{q}}\right), 114.9\left(\mathrm{CH}_{2}\right), 114.0(\mathrm{CH}), 113.5(\mathrm{CH}), 112.4(\mathrm{CH})$, 101.6 (CH), $56.0\left(\mathrm{CH}_{3}\right), 33.7\left(\mathrm{CH}_{2}\right), 29.0\left(\mathrm{CH}_{2}\right), 24.6\left(\mathrm{CH}_{2}\right), 18.0\left(\mathrm{CH}_{3}\right)$. IR (ATR): 2925, 2857, 1603, 1490, 1471, 1396, $790 \mathrm{~cm}^{-1}$. MS (ESI-TOF) $\mathrm{m} / z$ (relative intensity): $329(5)[\mathrm{M}+\mathrm{Na}]^{+}, 307$ (100) $[\mathrm{M}+\mathrm{H}]^{+}$. HR-MS (ESI-TOF): $m / z:[\mathrm{M}+\mathrm{H}]^{+}$Calcd. for $\mathrm{C}_{20} \mathrm{H}_{23} \mathrm{~N}_{2} \mathrm{O}$ 307.1805; Found 307.1809 .

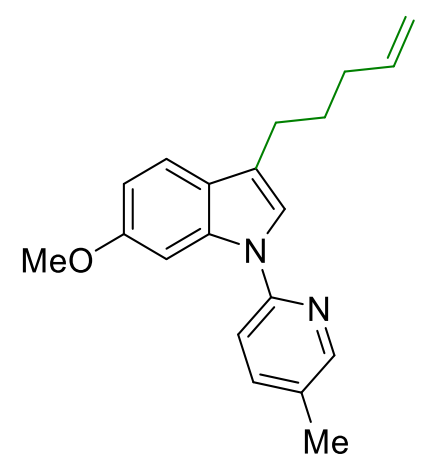

6-Methoxy-1-(5-methylpyridin-2-yl)-3-(pent-4-en-1-yl)-1 $\boldsymbol{H}$-indole (1e): Yellow oil (348 mg, $38 \%$ yield). ${ }^{1} \mathrm{H}-\mathrm{NMR}\left(400 \mathrm{MHz}, \mathrm{CDCl}_{3}\right): \delta=8.37(\mathrm{~d}, J=2.5 \mathrm{~Hz}, 1 \mathrm{H}), 7.78(\mathrm{~d}, J=2.4 \mathrm{~Hz}, 1 \mathrm{H})$, $7.59(\mathrm{dd}, J=8.4,2.4 \mathrm{~Hz}, 1 \mathrm{H}), 7.53-7.45(\mathrm{~m}, 1 \mathrm{H}), 7.35(\mathrm{~s}, 1 \mathrm{H}), 7.32(\mathrm{~d}, J=8.4 \mathrm{~Hz}, 1 \mathrm{H}), 6.86(\mathrm{dd}$, $J=8.7,2.4 \mathrm{~Hz}, 1 \mathrm{H}), 5.88(\mathrm{ddt}, J=16.9,9.4,6.7 \mathrm{~Hz}, 1 \mathrm{H}), 5.11-4.93(\mathrm{~m}, 2 \mathrm{H}), 3.89(\mathrm{~s}, 3 \mathrm{H}), 2.77(\mathrm{t}$, $J=7.6 \mathrm{~Hz}, 2 \mathrm{H}), 2.37$ (s, 3H), 2.19 (q, $J=7.0 \mathrm{~Hz}, 2 \mathrm{H}), 1.85$ (p, $J=7.6 \mathrm{~Hz}, 2 \mathrm{H}) .{ }^{13} \mathrm{C}-\mathrm{NMR}(100$ $\left.\mathrm{MHz}, \mathrm{CDCl}_{3}\right): \delta=157.2\left(\mathrm{C}_{\mathrm{q}}\right), 150.8\left(\mathrm{C}_{\mathrm{q}}\right), 148.9(\mathrm{CH}), 139.0(\mathrm{CH}), 138.9(\mathrm{CH}), 136.4\left(\mathrm{C}_{\mathrm{q}}\right), 129.0$ $\left(\mathrm{C}_{\mathrm{q}}\right), 124.6\left(\mathrm{C}_{\mathrm{q}}\right), 121.9(\mathrm{CH}), 119.7(\mathrm{CH}), 119.3\left(\mathrm{C}_{\mathrm{q}}\right), 114.8\left(\mathrm{CH}_{2}\right), 113.8(\mathrm{CH}), 110.0(\mathrm{CH}), 97.6$ (CH), $55.9\left(\mathrm{CH}_{3}\right), 33.8\left(\mathrm{CH}_{2}\right), 29.1\left(\mathrm{CH}_{2}\right), 24.7\left(\mathrm{CH}_{2}\right), 18.0\left(\mathrm{CH}_{3}\right)$. IR (ATR): 2927, 2954, 1601, 1477, 1394, 1210, $802 \mathrm{~cm}^{-1}$. MS (ESI-TOF) $\mathrm{m} / z$ (relative intensity): 307 (100) $[\mathrm{M}+\mathrm{H}]^{+}$. HR-MS (ESI-TOF): $m / z:[\mathrm{M}+\mathrm{H}]^{+}$Calcd. for $\mathrm{C}_{20} \mathrm{H}_{23} \mathrm{~N}_{2} \mathrm{O}$ 307.1805; Found 307.1815.

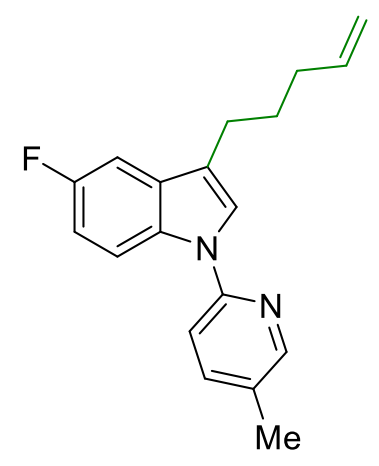

5-Fluoro-1-(5-methylpyridin-2-yl)-3-(pent-4-en-1-yl)-1H-indole (1f): Yellow oil (300 mg, 34\%). ${ }^{1} \mathrm{H}-\mathrm{NMR}\left(400 \mathrm{MHz}, \mathrm{CDCl}_{3}\right): \delta=8.38(\mathrm{~d}, J=3.5 \mathrm{~Hz}, 1 \mathrm{H}), 8.19(\mathrm{~d}, J=4.5 \mathrm{~Hz}, 1 \mathrm{H}), 7.60$ 
(dd, $J=8.3,2.4 \mathrm{~Hz}, 1 \mathrm{H}), 7.60-7.50(\mathrm{~m}, 2 \mathrm{H}), 7.38-7.20(\mathrm{~m}, 1 \mathrm{H}), 7.05(\mathrm{td}, J=9.1,2.5 \mathrm{~Hz}, 1 \mathrm{H})$, $5.92(\mathrm{ddt}, J=17.0,10.1,6.6 \mathrm{~Hz}, 1 \mathrm{H}), 5.21-5.01(\mathrm{~m}, 2 \mathrm{H}), 2.78(\mathrm{t}, J=7.7 \mathrm{~Hz}, 2 \mathrm{H}), 2.39(\mathrm{~s}, 3 \mathrm{H})$, $2.23(\mathrm{q}, J=7.3 \mathrm{~Hz}, 2 \mathrm{H}), 1.88(\mathrm{p}, J=7.6 \mathrm{~Hz}, 2 \mathrm{H}) .{ }^{13} \mathrm{C}-\mathrm{NMR}\left(100 \mathrm{MHz}, \mathrm{CDCl}_{3}\right): \delta=158.3\left(\mathrm{~d},{ }^{1} J_{\mathrm{C}-}\right.$ $\left.\mathrm{F}=236.5 \mathrm{~Hz}, \mathrm{C}_{\mathrm{q}}\right), 150.4\left(\mathrm{C}_{\mathrm{q}}\right), 148.9(\mathrm{CH}), 139.1(\mathrm{CH}), 138.7(\mathrm{CH}), 132.1\left(\mathrm{C}_{\mathrm{q}}\right), 130.9\left(\mathrm{~d},{ }^{3} J_{\mathrm{C}-\mathrm{F}}=\right.$ $\left.9.3 \mathrm{~Hz}, \mathrm{C}_{\mathrm{q}}\right), 129.2\left(\mathrm{C}_{\mathrm{q}}\right), 124.4(\mathrm{CH}), 119.0\left(\mathrm{~d},{ }^{4} J_{\mathrm{C}-\mathrm{F}}=4.4 \mathrm{~Hz}, \mathrm{C}_{\mathrm{q}}\right), 114.9\left(\mathrm{CH}_{2}\right), 114.2\left(\mathrm{~d},{ }^{3} J_{\mathrm{C}-\mathrm{F}}=\right.$ $9.2 \mathrm{~Hz}, \mathrm{CH}), 113.5(\mathrm{CH}), 111.0\left(\mathrm{~d},{ }^{2} J_{\mathrm{C}-\mathrm{F}}=25.4 \mathrm{~Hz}, \mathrm{CH}\right), 104.3\left(\mathrm{~d},{ }^{2} J_{\mathrm{C}-\mathrm{F}}=23.1 \mathrm{~Hz}, \mathrm{CH}\right), 33.7$ $\left(\mathrm{CH}_{2}\right), 28.9\left(\mathrm{CH}_{2}\right), 24.5\left(\mathrm{CH}_{2}\right), 17.9\left(\mathrm{CH}_{3}\right) .{ }^{19} \mathrm{~F}-\mathrm{NMR}\left(282 \mathrm{MHz}, \mathrm{CDCl}_{3}\right): \delta=-123.2$. IR (ATR): 2926, 2856, 1602, 1471, 1437, 1395, 1132, 911, $789 \mathrm{~cm}^{-1}$. MS (ESI-TOF) $\mathrm{m} / \mathrm{z}$ (relative intensity): $317(20)[\mathrm{M}+\mathrm{Na}]^{+}, 295(100)[\mathrm{M}+\mathrm{H}]^{+}$. HR-MS (ESI-TOF) $\mathrm{m} / z:[\mathrm{M}+\mathrm{H}]^{+}$Calcd. for $\mathrm{C}_{19} \mathrm{H}_{20}{ }^{19} \mathrm{FN}_{2}$ 295.1605; Found 295.1613.

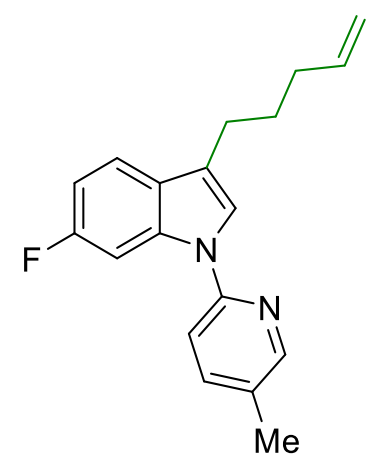

6-Fluoro-1-(5-methylpyridin-2-yl)-3-(pent-4-en-1-yl)-1H-indole (1g): Yellow oil (414 mg, $47 \%$ yield). ${ }^{1} \mathrm{H}-\mathrm{NMR}\left(400 \mathrm{MHz}, \mathrm{CDCl}_{3}\right): \delta=8.36(\mathrm{~d}, J=2.4 \mathrm{~Hz}, 1 \mathrm{H}), 7.98(\mathrm{dd}, J=10.8,2.3 \mathrm{~Hz}$, 1H), $7.60(\mathrm{dd}, J=8.3,2.4 \mathrm{~Hz}, 1 \mathrm{H}), 7.51(\mathrm{dd}, J=8.7,5.5 \mathrm{~Hz}, 1 \mathrm{H}), 7.42(\mathrm{~d}, J=1.4 \mathrm{~Hz}, 1 \mathrm{H}), 7.29$ $(\mathrm{d}, J=8.3 \mathrm{~Hz}, 1 \mathrm{H}), 6.96(\mathrm{td}, J=9.0,2.3 \mathrm{~Hz}, 1 \mathrm{H}), 5.88(\mathrm{ddt}, J=16.8,10.0,6.6 \mathrm{~Hz}, 1 \mathrm{H}), 5.13-4.94$ (m, 2H), $2.78(\mathrm{t}, J=7.7 \mathrm{~Hz}, 2 \mathrm{H}), 2.37(\mathrm{~s}, 3 \mathrm{H}), 2.19(\mathrm{q}, J=7.8 \mathrm{~Hz}, 2 \mathrm{H}), 1.85(\mathrm{p}, J=7.6 \mathrm{~Hz}, 2 \mathrm{H})$. ${ }^{13} \mathrm{C}-\mathrm{NMR}\left(101 \mathrm{MHz}, \mathrm{CDCl}_{3}\right): \delta=160.6\left(\mathrm{~d},{ }^{1} J_{\mathrm{C}-\mathrm{F}}=234.1 \mathrm{~Hz}, \mathrm{C}_{\mathrm{q}}\right), 150.5\left(\mathrm{C}_{\mathrm{q}}\right), 149.0(\mathrm{CH}), 139.1$ $(\mathrm{CH}), 138.7(\mathrm{CH}), 135.6\left(\mathrm{~d},{ }^{3} J_{\mathrm{C}-\mathrm{F}}=12.2 \mathrm{~Hz}, \mathrm{C}_{\mathrm{q}}\right) 129.4\left(\mathrm{C}_{\mathrm{q}}\right), 126.7(\mathrm{CH}), 123.0\left(\mathrm{~d},{ }^{3} J_{\mathrm{C}-\mathrm{F}}=4.1 \mathrm{~Hz}\right.$, $\mathrm{CH}), 119.8\left(\mathrm{~d},{ }^{3} J_{\mathrm{C}-\mathrm{F}}=10.2 \mathrm{~Hz}, \mathrm{CH}\right), 119.3\left(\mathrm{C}_{\mathrm{q}}\right), 114.9\left(\mathrm{CH}_{2}\right), 113.5(\mathrm{CH}), 109.1\left(\mathrm{~d},{ }^{2} J_{\mathrm{C}-\mathrm{F}}=24.1\right.$ $\mathrm{Hz}, \mathrm{CH}), 100.5\left(\mathrm{~d},{ }^{2} J_{\mathrm{C}-\mathrm{F}}=27.2 \mathrm{~Hz}, \mathrm{CH}\right), 33.7\left(\mathrm{CH}_{2}\right), 29.1\left(\mathrm{CH}_{2}\right), 24.6\left(\mathrm{CH}_{2}\right), 17.9\left(\mathrm{CH}_{3}\right),{ }^{19} \mathrm{~F}-$ NMR (377 MHz, $\mathrm{CDCl}_{3}$ ): $\delta=-119.6$. IR (ATR): 2927, 1600, 1490, 1475, 1443, 1392, $802 \mathrm{~cm}^{-1}$. MS (ESI-TOF) $\mathrm{m} / z$ (relative intensity): $317(15)[\mathrm{M}+\mathrm{Na}]^{+}, 295$ (100) $[\mathrm{M}+\mathrm{H}]^{+}$. HR-MS (ESITOF): $m / z:[\mathrm{M}+\mathrm{H}]^{+}$Calcd. for $\mathrm{C}_{19} \mathrm{H}_{20}{ }^{19} \mathrm{FN}_{2}$ 295.1605; Found 295.1607. 


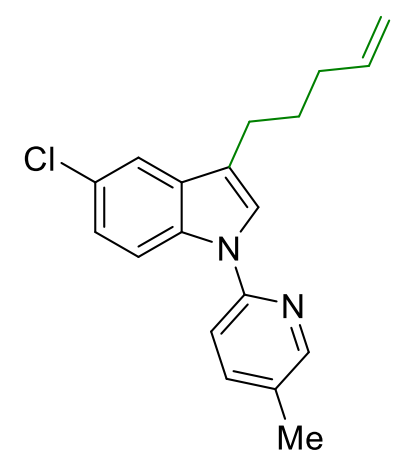

5-Chloro-1-(5-methylpyridin-2-yl)-3-(pent-4-en-1-yl)-1H-indole (1h): Yellow waxy solid (476 mg, 51\%). ${ }^{1} \mathrm{H}-\mathrm{NMR}\left(400 \mathrm{MHz}, \mathrm{CDCl}_{3}\right): \delta=8.36(\mathrm{~d}, J=2.7 \mathrm{~Hz}, 1 \mathrm{H}), 8.11(\mathrm{~d}, J=8.8 \mathrm{~Hz}, 1 \mathrm{H})$, 7.63-7.58 (m, 1H), 7.57 (s, 1H), $7.47(\mathrm{~s}, 1 \mathrm{H}), 7.31$ (d, J=8.3 Hz, 1H), 7.22 (dd, J=8.8, 2.2 Hz, $1 \mathrm{H}), 5.88(\mathrm{ddt}, J=16.8,9.7,6.6 \mathrm{~Hz}, 1 \mathrm{H}), 5.11-4.97(\mathrm{~m}, 2 \mathrm{H}), 2.77(\mathrm{t}, J=7.7 \mathrm{~Hz}, 2 \mathrm{H}), 2.37(\mathrm{~s}, 3 \mathrm{H})$, $2.19(\mathrm{q}, J=7.2 \mathrm{~Hz}, 2 \mathrm{H}), 1.84(\mathrm{p}, J=7.6 \mathrm{~Hz}, 2 \mathrm{H}) .{ }^{13} \mathrm{C}$ NMR One carbon resonance is missing due to overlap $\left(100 \mathrm{MHz}, \mathrm{CDCl}_{3}\right): \delta=150.3\left(\mathrm{C}_{\mathrm{q}}\right), 149.0(\mathrm{CH}), 139.1(\mathrm{CH}), 138.7(\mathrm{CH}), 133.9\left(\mathrm{C}_{\mathrm{q}}\right)$, 131.4 $\left(\mathrm{C}_{\mathrm{q}}\right), 129.5\left(\mathrm{C}_{\mathrm{q}}\right), 126.3\left(\mathrm{C}_{\mathrm{q}}\right), 124.2(\mathrm{CH}), 123.2(\mathrm{CH}), 118.8(\mathrm{CH}), 115.0\left(\mathrm{CH}_{2}\right), 114.3(\mathrm{CH})$, $113.8(\mathrm{CH}), 33.7\left(\mathrm{CH}_{2}\right), 29.0\left(\mathrm{CH}_{2}\right), 24.5\left(\mathrm{CH}_{2}\right), 17.9\left(\mathrm{CH}_{2}\right)$. IR (ATR): 2926, 2856, 1601, 1487, 1454, 1217, 911, $720 \mathrm{~cm}^{-1}$. MS (ESI-TOF) $\mathrm{m} / z$ (relative intensity): $311(100)[\mathrm{M}+\mathrm{H}]^{+}$. HR-MS (ESI-TOF) $m / z:[\mathrm{M}+\mathrm{H}]^{+}$Calcd. for $\mathrm{C}_{19} \mathrm{H}_{20}{ }^{35} \mathrm{ClN}_{2}$ 311.1310; Found 311.1306.

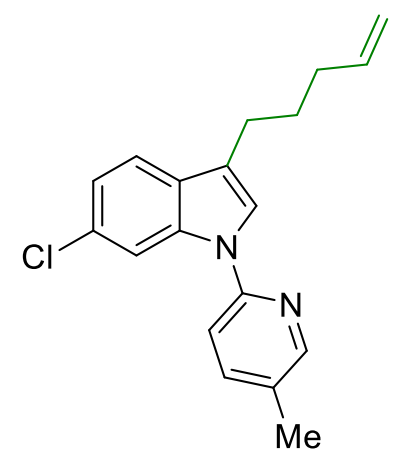

6-Chloro-1-(5-methylpyridin-2-yl)-3-(pent-4-en-1-yl)-1H-indole (1i): Yellow waxy solid (448 mg, 48\%). ${ }^{1} \mathrm{H}-\mathrm{NMR}\left(400 \mathrm{MHz}, \mathrm{CDCl}_{3}\right): \delta=8.37$ (d, $\left.J=2.4 \mathrm{~Hz}, 1 \mathrm{H}\right), 8.23$ (s, 1H), 7.61 (dd, $J=$ 8.4, $2.3 \mathrm{~Hz}, 1 \mathrm{H}), 7.50(\mathrm{~d}, J=8.4 \mathrm{~Hz}, 1 \mathrm{H}), 7.43(\mathrm{~s}, 1 \mathrm{H}), 7.30(\mathrm{~d}, J=8.3 \mathrm{~Hz}, 1 \mathrm{H}), 7.16(\mathrm{~d}, J=8.4$ Hz, 1H), 5.87 (ddt, $J=16.9,9.8,6.6 \mathrm{~Hz}, 1 \mathrm{H}), 5.10-4.97(\mathrm{~m}, 2 \mathrm{H}), 2.77$ (t, $J=7.7 \mathrm{~Hz}, 2 \mathrm{H}), 2.38$ (s, 3H), 2.18 (q, $J=7.3 \mathrm{~Hz}, 2 \mathrm{H}), 1.84$ (p, $J=7.6 \mathrm{~Hz}, 2 \mathrm{H}) .{ }^{13} \mathrm{C}-\mathrm{NMR}\left(100 \mathrm{MHz}, \mathrm{CDCl}_{3}\right): \delta=150.3$ $\left(\mathrm{C}_{\mathrm{q}}\right), 149.1(\mathrm{CH}), 139.2(\mathrm{CH}), 138.7(\mathrm{CH}), 135.9\left(\mathrm{C}_{\mathrm{q}}\right), 129.6\left(\mathrm{C}_{\mathrm{q}}\right), 129.1\left(\mathrm{C}_{\mathrm{q}}\right), 128.7\left(\mathrm{C}_{\mathrm{q}}\right), 123.5$ $(\mathrm{CH}), 121.2(\mathrm{CH}), 119.9(\mathrm{CH}), 119.3\left(\mathrm{C}_{\mathrm{q}}\right), 114.9\left(\mathrm{CH}_{2}\right), 113.9(\mathrm{CH}), 113.4(\mathrm{CH}), 33.7\left(\mathrm{CH}_{2}\right), 29.1$ $\left(\mathrm{CH}_{2}\right), 24.5\left(\mathrm{CH}_{2}\right), 17.9\left(\mathrm{CH}_{3}\right)$. IR (ATR): 2927, 2858, 1600, 1489, 1438, 1391, 1213, $800 \mathrm{~cm}^{-1}$. 
MS (ESI-TOF) $m / z$ (relative intensity): $311(100)[\mathrm{M}+\mathrm{H}]^{+}$. HR-MS (ESI-TOF) $m / z:[\mathrm{M}+\mathrm{H}]^{+}$ Calcd. for $\mathrm{C}_{19} \mathrm{H}_{20}{ }^{35} \mathrm{ClN}_{2}$ 311.1310; Found 311.1304.

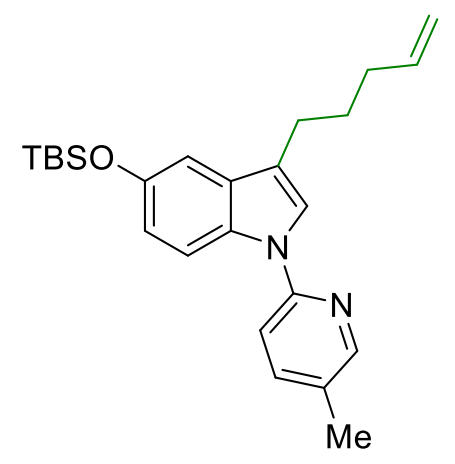

5-[(tert-Butyldimethylsilyl)oxy]-1-(5-methylpyridin-2-yl)-3-(pent-4-en-1-yl)-1H-indole (1j): Brown waxy solid (647 mg, 53\%). ${ }^{1} \mathrm{H}-\mathrm{NMR}\left(400 \mathrm{MHz}, \mathrm{CDCl}_{3}\right): \delta=8.33(\mathrm{~s}, 1 \mathrm{H}), 8.03(\mathrm{~d}, J=8.9$ $\mathrm{Hz}, 1 \mathrm{H}), 7.57$ (dd, $J=8.3,2.5 \mathrm{~Hz}, 1 \mathrm{H}), 7.45$ (s, 1H), 7.32 (d, $J=8.3 \mathrm{~Hz}, 1 \mathrm{H}), 7.02$ (d, $J=2.3 \mathrm{~Hz}$, $1 \mathrm{H}), 6.83(\mathrm{dd}, J=8.9,2.4 \mathrm{~Hz}, 1 \mathrm{H}), 5.89(\mathrm{ddt}, J=16.8,10.0,6.6 \mathrm{~Hz}, 1 \mathrm{H}), 5.12-4.95(\mathrm{~m}, 2 \mathrm{H}), 2.75$ (t, $J=7.6 \mathrm{~Hz}, 2 \mathrm{H}), 2.36$ (s, 3H), 2.19 (q, $J=7.4 \mathrm{~Hz}, 2 \mathrm{H}), 1.85$ (p, $J=7.6 \mathrm{~Hz}, 2 \mathrm{H}), 1.03$ (s, 9H), $0.23(\mathrm{~s}, 6 \mathrm{H}) .{ }^{13} \mathrm{C}-\mathrm{NMR}\left(100 \mathrm{MHz}, \mathrm{CDCl}_{3}\right): \delta=150.7\left(\mathrm{C}_{\mathrm{q}}\right), 150.0\left(\mathrm{C}_{\mathrm{q}}\right), 148.9(\mathrm{CH}), 138.9(\mathrm{CH})$, $138.9(\mathrm{CH}), 131.2\left(\mathrm{C}_{\mathrm{q}}\right), 131.1\left(\mathrm{C}_{\mathrm{q}}\right), 128.7\left(\mathrm{C}_{\mathrm{q}}\right), 123.5(\mathrm{CH}), 118.8\left(\mathrm{C}_{\mathrm{q}}\right), 116.8(\mathrm{CH}), 114.8\left(\mathrm{CH}_{2}\right)$, 113.6 (CH), $113.4(\mathrm{CH}), 109.1(\mathrm{CH}), 33.7\left(\mathrm{CH}_{2}\right), 29.0\left(\mathrm{CH}_{2}\right), 25.9\left(\mathrm{CH}_{3}\right), 24.6\left(\mathrm{CH}_{2}\right), 18.4\left(\mathrm{C}_{\mathrm{q}}\right)$, $17.9\left(\mathrm{CH}_{3}\right),-4.2\left(\mathrm{CH}_{3}\right)$. IR (ATR): 2927, 2857, 1603, 1468, 1446, 1255, 969, $837 \mathrm{~cm}^{-1}$. MS (ESITOF) $m / z$ (relative intensity): $836(5)[2 \mathrm{M}+\mathrm{Na}]^{+}, 814(20)[2 \mathrm{M}+\mathrm{H}]^{+}, 407(100)[\mathrm{M}+\mathrm{H}]^{+} . \mathrm{HR}-$ MS (ESI-TOF) $m / z:[\mathrm{M}+\mathrm{H}]^{+}$Calcd. for $\mathrm{C}_{25} \mathrm{H}_{35} \mathrm{~N}_{2} \mathrm{OSi}$ 407.2513; Found 407.2512.

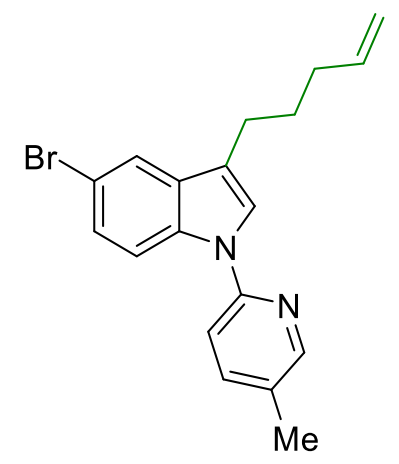

5-Bromo-1-(5-methylpyridin-2-yl)-3-(pent-4-en-1-yl)-1H-indole (1k): Yellow oil (541 mg, $51 \%$ yield). ${ }^{1} \mathrm{H}-\mathrm{NMR}\left(300 \mathrm{MHz}, \mathrm{CDCl}_{3}\right): \delta=8.36(\mathrm{~d}, J=2.4 \mathrm{~Hz}, 1 \mathrm{H}), 8.05(\mathrm{~d}, J=8.8 \mathrm{~Hz}, 1 \mathrm{H})$, $7.72(\mathrm{~d}, J=2.0 \mathrm{~Hz}, 1 \mathrm{H}), 7.61(\mathrm{dd}, J=8.2,2.4 \mathrm{~Hz}, 1 \mathrm{H}), 7.46(\mathrm{~s}, 1 \mathrm{H}), 7.37-7.29(\mathrm{~m}, 2 \mathrm{H}), 5.87$ (ddt, $J=16.9,10.1,6.6 \mathrm{~Hz}, 1 \mathrm{H}), 5.15-4.96(\mathrm{~m}, 2 \mathrm{H}), 2.75(\mathrm{t}, J=7.7 \mathrm{~Hz}, 2 \mathrm{H}), 2.38(\mathrm{~s}, 3 \mathrm{H}), 2.18(\mathrm{q}, J=$ $7.1 \mathrm{~Hz}, 2 \mathrm{H}), 1.84(\mathrm{p}, J=7.5 \mathrm{~Hz}, 2 \mathrm{H}) .{ }^{13} \mathrm{C}-\mathrm{NMR}\left(100 \mathrm{MHz}, \mathrm{CDCl}_{3}\right): \delta=150.3\left(\mathrm{C}_{\mathrm{q}}\right), 149.1(\mathrm{CH})$, 
139.2 $(\mathrm{CH}), 138.7(\mathrm{CH}), 134.3\left(\mathrm{C}_{\mathrm{q}}\right), 132.0\left(\mathrm{C}_{\mathrm{q}}\right), 129.6\left(\mathrm{C}_{\mathrm{q}}\right), 125.8(\mathrm{CH}), 124.1(\mathrm{CH}), 121.9(\mathrm{CH})$, $118.7\left(\mathrm{C}_{\mathrm{q}}\right), 115.0(\mathrm{CH}), 114.7\left(\mathrm{CH}_{2}\right), 113.9(\mathrm{CH}), 113.9(\mathrm{CH}), 33.7\left(\mathrm{CH}_{2}\right), 29.0\left(\mathrm{CH}_{2}\right), 24.4\left(\mathrm{CH}_{2}\right)$, $18.0\left(\mathrm{CH}_{3}\right)$. IR (ATR): 2927, 1601, 1488, 1453, 1395, $789 \mathrm{~cm}^{-1}$. MS (ESI-TOF) $\mathrm{m} / z$ (relative intensity): 355 (100) $[\mathrm{M}+\mathrm{H}]^{+}$. HR-MS (ESI-TOF): $m / z$ : $[\mathrm{M}+\mathrm{H}]^{+}$Calcd. for $\mathrm{C}_{19} \mathrm{H}_{20}{ }^{79} \mathrm{BrN}_{2}$ 355.0804; Found 355.0807.

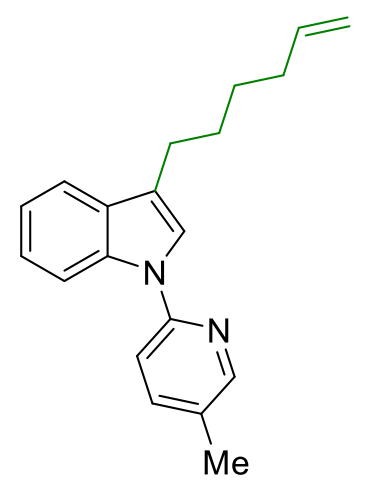

3-(Hex-5-en-1-yl)-1-(5-methylpyridin-2-yl)-1H-indole (3a): Yellow oil (479 mg, 55\%). ${ }^{1} \mathrm{H}$ NMR (400 MHz, $\left.\mathrm{CDCl}_{3}\right): \delta=8.37(\mathrm{~d}, J=2.8 \mathrm{~Hz}, 1 \mathrm{H}), 8.12(\mathrm{~d}, J=8.3 \mathrm{~Hz}, 1 \mathrm{H}), 7.62(\mathrm{t}, J=7.5$ $\mathrm{Hz}, 2 \mathrm{H}), 7.50$ (s, 1H), $7.38(\mathrm{~d}, J=8.3 \mathrm{~Hz}, 1 \mathrm{H}), 7.29(\mathrm{~d}, J=7.5 \mathrm{~Hz}, 1 \mathrm{H}), 7.20$ (t, $J=7.5 \mathrm{~Hz}, 1 \mathrm{H})$, $5.84(\mathrm{ddt}, J=17.0,10.1,6.7 \mathrm{~Hz}, 1 \mathrm{H}), 5.17-4.85(\mathrm{~m}, 2 \mathrm{H}), 2.80(\mathrm{t}, J=7.6 \mathrm{~Hz}, 2 \mathrm{H}), 2.37$ (s, 3H), $2.13(\mathrm{q}, J=7.2 \mathrm{~Hz}, 2 \mathrm{H}), 1.79$ (p, $J=7.7 \mathrm{~Hz}, 2 \mathrm{H}), 1.55$ (p, $J=7.5 \mathrm{~Hz}, 2 \mathrm{H}) .{ }^{13} \mathrm{C}-\mathrm{NMR}(100 \mathrm{MHz}$, $\left.\mathrm{CDCl}_{3}\right): \delta=150.6\left(\mathrm{C}_{\mathrm{q}}\right), 149.1(\mathrm{CH}), 139.1(\mathrm{CH}), 139.0(\mathrm{CH}), 135.5\left(\mathrm{C}_{\mathrm{q}}\right), 130.3\left(\mathrm{C}_{\mathrm{q}}\right), 129.2\left(\mathrm{C}_{\mathrm{q}}\right)$, $123.1(\mathrm{CH}), 123.0(\mathrm{CH}), 120.6(\mathrm{CH}), 119.4\left(\mathrm{C}_{\mathrm{q}}\right), 119.3(\mathrm{CH}), 114.5\left(\mathrm{CH}_{2}\right), 114.1(\mathrm{CH}), 112.8$ $(\mathrm{CH}), 33.8\left(\mathrm{CH}_{2}\right), 29.4\left(\mathrm{CH}_{2}\right), 29.0\left(\mathrm{CH}_{2}\right), 25.1\left(\mathrm{CH}_{2}\right), 18.0\left(\mathrm{CH}_{3}\right)$. IR (ATR): 2925, 2855, 1601, 1486, 1436, 1216, 817, $790 \mathrm{~cm}^{-1}$. MS (ESI-TOF) $\mathrm{m} / \mathrm{z}$ (relative intensity): $291(100)[\mathrm{M}+\mathrm{H}]^{+}$. HRMS (ESI-TOF): $m / z$ [M + H] ${ }^{+}$Calcd. for $\mathrm{C}_{20} \mathrm{H}_{23} \mathrm{~N}_{2}$ 291.1856; Found 291.1860.

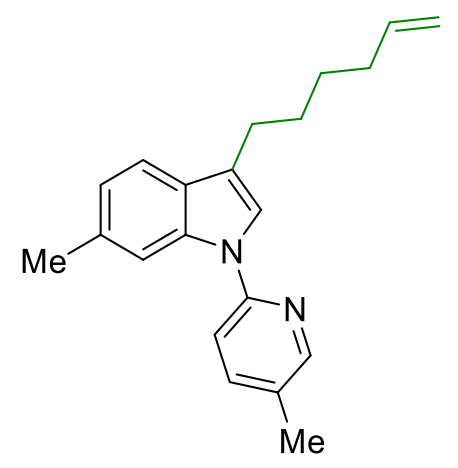

3-(Hex-5-en-1-yl)-6-methyl-1-(5-methylpyridin-2-yl)-1H-indole (3b): Yellow oil (375 mg, $41 \%$ yield). ${ }^{1} \mathrm{H}-\mathrm{NMR}\left(400 \mathrm{MHz}, \mathrm{CDCl}_{3}\right): \delta=8.37$ (d, $\left.J=2.4 \mathrm{~Hz}, 1 \mathrm{H}\right), 7.92(\mathrm{~s}, 1 \mathrm{H}), 7.60$ (dd, $J=$ 
8.3, 2.4 Hz, 1H), $7.49(\mathrm{~d}, J=8.0 \mathrm{~Hz}, 1 \mathrm{H}), 7.40(\mathrm{~s}, 1 \mathrm{H}), 7.36(\mathrm{~d}, J=8.3 \mathrm{~Hz}, 1 \mathrm{H}), 7.02(\mathrm{~d}, J=8.0$ Hz, 1H), 5.83 (ddt, $J=17.0,10.1,6.7 \mathrm{~Hz}, 1 \mathrm{H}), 5.07-4.79$ (m, 2H), 2.77 (t, $J=7.6 \mathrm{~Hz}, 2 \mathrm{H}), 2.50$ (s, 3H), 2.37 (s, 3H), 2.12 (q, $J=7.2 \mathrm{~Hz}, 2 \mathrm{H}), 1.77$ (p, $J=7.7 \mathrm{~Hz}, 2 \mathrm{H}), 1.61-1.49$ (m, 2H). ${ }^{13} \mathrm{C}-$ $\operatorname{NMR}\left(100 \mathrm{MHz}, \mathrm{CDCl}_{3}\right): \delta=150.7\left(\mathrm{C}_{\mathrm{q}}\right), 149.0(\mathrm{CH}), 139.2(\mathrm{CH}), 139.0(\mathrm{CH}), 135.9\left(\mathrm{C}_{\mathrm{q}}\right), 132.9$ $\left(\mathrm{C}_{\mathrm{q}}\right), 129.0\left(\mathrm{C}_{\mathrm{q}}\right), 128.2\left(\mathrm{C}_{\mathrm{q}}\right), 122.5(\mathrm{CH}), 122.2(\mathrm{CH}), 119.4\left(\mathrm{C}_{\mathrm{q}}\right), 119.0(\mathrm{CH}), 114.5\left(\mathrm{CH}_{2}\right), 114.2$ $(\mathrm{CH}), 112.8(\mathrm{CH}), 33.8\left(\mathrm{CH}_{2}\right), 29.4\left(\mathrm{CH}_{2}\right), 29.0\left(\mathrm{CH}_{2}\right), 25.1\left(\mathrm{CH}_{2}\right), 22.2\left(\mathrm{CH}_{3}\right), 18.0\left(\mathrm{CH}_{3}\right)$. IR (ATR): 2924, 1601, 1573, 1493, 1433, 1382, $803 \mathrm{~cm}^{-1}$. MS (ESI-TOF) $\mathrm{m} / \mathrm{z}$ (relative intensity): $305(100)[\mathrm{M}+\mathrm{H}]^{+}$. HR-MS (ESI-TOF) $m / z:[\mathrm{M}+\mathrm{H}]^{+}$Calcd. for $\mathrm{C}_{21} \mathrm{H}_{25} \mathrm{~N}_{2}$ 305.2012; Found 305.2014 .

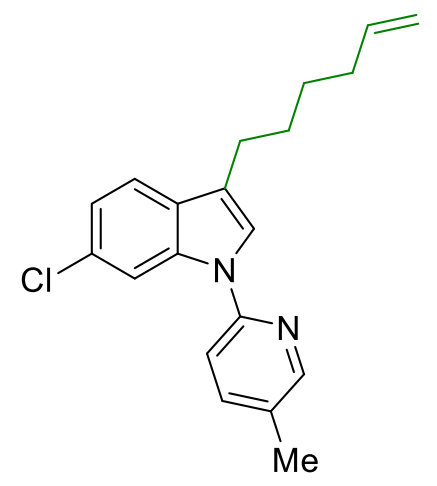

6-Chloro-3-(hex-5-en-1-yl)-1-(5-methylpyridin-2-yl)-1H-indole (3c): Yellow oil (439 mg, 45\%). ${ }^{1} \mathrm{H}-\mathrm{NMR}\left(400 \mathrm{MHz}, \mathrm{CDCl}_{3}\right): \delta=8.35(\mathrm{~d}, J=2.3 \mathrm{~Hz}, 1 \mathrm{H}), 8.09(\mathrm{~d}, J=8.7 \mathrm{~Hz}, 1 \mathrm{H}), 7.61(\mathrm{~d}$, $J=8.4 \mathrm{~Hz}, 1 \mathrm{H}), 7.56(\mathrm{~s}, 1 \mathrm{H}), 7.46(\mathrm{~s}, 1 \mathrm{H}), 7.31(\mathrm{~d}, J=8.2 \mathrm{~Hz}, 1 \mathrm{H}), 7.21(\mathrm{~d}, J=8.8 \mathrm{~Hz}, 1 \mathrm{H}), 5.83$ (ddt, $J=17.0,9.3,6.5 \mathrm{~Hz}, 1 \mathrm{H}), 5.12-4.92(\mathrm{~m}, 2 \mathrm{H}), 2.74(\mathrm{t}, J=7.7 \mathrm{~Hz}, 2 \mathrm{H}), 2.37(\mathrm{~s}, 3 \mathrm{H}), 2.12(\mathrm{q}$, $J=7.2 \mathrm{~Hz}, 2 \mathrm{H}), 1.75(\mathrm{p}, J=7.7 \mathrm{~Hz}, 2 \mathrm{H}), 1.52(\mathrm{p}, J=7.5 \mathrm{~Hz}, 2 \mathrm{H}) .{ }^{13} \mathrm{C}-\mathrm{NMR}\left(100 \mathrm{MHz}, \mathrm{CDCl}_{3}\right)$ : $\delta=150.3\left(\mathrm{C}_{\mathrm{q}}\right), 149.0(\mathrm{CH}), 139.2(\mathrm{CH}), 139.0(\mathrm{CH}), 133.9\left(\mathrm{C}_{\mathrm{q}}\right), 131.4\left(\mathrm{C}_{\mathrm{q}}\right), 129.5\left(\mathrm{C}_{\mathrm{q}}\right), 126.2\left(\mathrm{C}_{\mathrm{q}}\right)$, 124.2 $(\mathrm{CH}), 123.2(\mathrm{CH}), 119.0\left(\mathrm{C}_{\mathrm{q}}\right), 118.8(\mathrm{CH}), 114.6\left(\mathrm{CH}_{2}\right), 114.3(\mathrm{CH}), 113.9(\mathrm{CH}), 33.8\left(\mathrm{CH}_{2}\right)$, $29.3\left(\mathrm{CH}_{2}\right), 28.9\left(\mathrm{CH}_{2}\right), 24.9\left(\mathrm{CH}_{2}\right), 17.9\left(\mathrm{CH}_{3}\right)$. IR (ATR): 2926, 2854, 1601, 1488, 1395, 1216, $741 \mathrm{~cm}^{-1}$. MS (ESI-TOF) $\mathrm{m} / z$ (relative intensity): $347(10)[\mathrm{M}+\mathrm{Na}]^{+}, 325(100)[\mathrm{M}+\mathrm{H}]^{+}$. HRMS (ESI-TOF) $m / z:[\mathrm{M}+\mathrm{H}]^{+}$Calcd. for $\mathrm{C}_{20} \mathrm{H}_{22}{ }^{35} \mathrm{ClN}_{2}$ 325.1466; Found 325.1471. 


\section{Characterization Data of Products 2}<smiles>[M]C1CCCc2c1n(-c1ccccn1)c1ccccc21</smiles>

(R)-1-Methyl-9-(pyridin-2-yl)-2,3,4,9-tetrahydro-1H-carbazole (2a'): The general procedure A was followed using 3-(pent-4-en-1-yl)-1-(pyridin-2-yl)-1H-indole (1a') (65.5 mg, 0.25 mmol). Isolation by column chromatography ( $n$-hexane/EtOAc $=20: 1)$ yielded $\mathbf{2 a}$ ' $(57.7 \mathrm{mg}, 88 \%)$ as a yellow solid. M.p.: 77-82 ${ }^{\circ} \mathrm{C} .{ }^{1} \mathrm{H}-\mathrm{NMR}\left(400 \mathrm{MHz} \mathrm{CDCl}_{3}\right): \delta=8.62(\mathrm{ddd}, J=4.9,2.0,0.9 \mathrm{~Hz}$, $1 \mathrm{H}), 7.85(\mathrm{ddd}, J=8.0,7.4,2.0 \mathrm{~Hz}, 1 \mathrm{H}), 7.52-7.49$ (m, 1H), $7.45(\mathrm{dd}, J=8.0,0.9 \mathrm{~Hz}, 1 \mathrm{H}), 7.40$ 7.37 (m, 1H), 7.26 (ddd, $J=7.4,4.9,0.9 \mathrm{~Hz}, 1 \mathrm{H}), 7.16-7.10$ (m, 2H), 3.57-3.47 (m, 1H), 2.84$2.67(\mathrm{~m}, 2 \mathrm{H}), 2.16-2.05(\mathrm{~m}, 1 \mathrm{H}), 1.98-1.81(\mathrm{~m}, 2 \mathrm{H}), 1.69-1.58(\mathrm{~m}, 1 \mathrm{H}), 0.83(\mathrm{~d}, J=6.9 \mathrm{~Hz}, 3 \mathrm{H})$. ${ }^{13} \mathrm{C}-\mathrm{NMR}\left(100 \mathrm{MHz}, \mathrm{CDCl}_{3}\right): \delta=152.1\left(\mathrm{C}_{\mathrm{q}}\right), 149.5(\mathrm{CH}), 140.2\left(\mathrm{C}_{\mathrm{q}}\right), 138.2(\mathrm{CH}), 136.8\left(\mathrm{C}_{\mathrm{q}}\right)$, $128.3\left(\mathrm{C}_{\mathrm{q}}\right), 121.8(\mathrm{CH}), 121.4(\mathrm{CH}), 120.2(\mathrm{CH}), 120.2(\mathrm{CH}), 118.1(\mathrm{CH}), 112.6\left(\mathrm{C}_{\mathrm{q}}\right), 109.8(\mathrm{CH})$, $31.9\left(\mathrm{CH}_{2}\right), 27.2(\mathrm{CH}), 21.5\left(\mathrm{CH}_{2}\right), 20.3\left(\mathrm{CH}_{3}\right), 19.9\left(\mathrm{CH}_{2}\right)$. IR (ATR): 2959, 2929, 1584, 1471, 1437, 1372, $738 \mathrm{~cm}^{-1}$. MS (ESI-TOF) $\mathrm{m} / z$ (relative intensity): $285(25)[\mathrm{M}+\mathrm{Na}]^{+}, 263(100)[\mathrm{M}$ $+\mathrm{H}]^{+}$. HR-MS (ESI-TOF) $m / z:[\mathrm{M}+\mathrm{H}]^{+}$Calcd. for $\mathrm{C}_{18} \mathrm{H}_{19} \mathrm{~N}_{2}$ 263.1543; Found 263.1542. $[\alpha]_{\mathrm{D}}^{20}$ : -70.5 (c = 1.0, $\left.\mathrm{CHCl}_{3}\right)$. HPLC separation (Chiralpak ${ }^{\circledR} \mathrm{IF}-3, n$-hexane/EtOAc 95:5, $1.0 \mathrm{~mL} / \mathrm{min}$, detection at $273 \mathrm{~nm}): t_{r}($ major $)=11.7 \mathrm{~min}, t_{r}($ minor $)=10.5 \mathrm{~min}, 76: 24$ e.r.

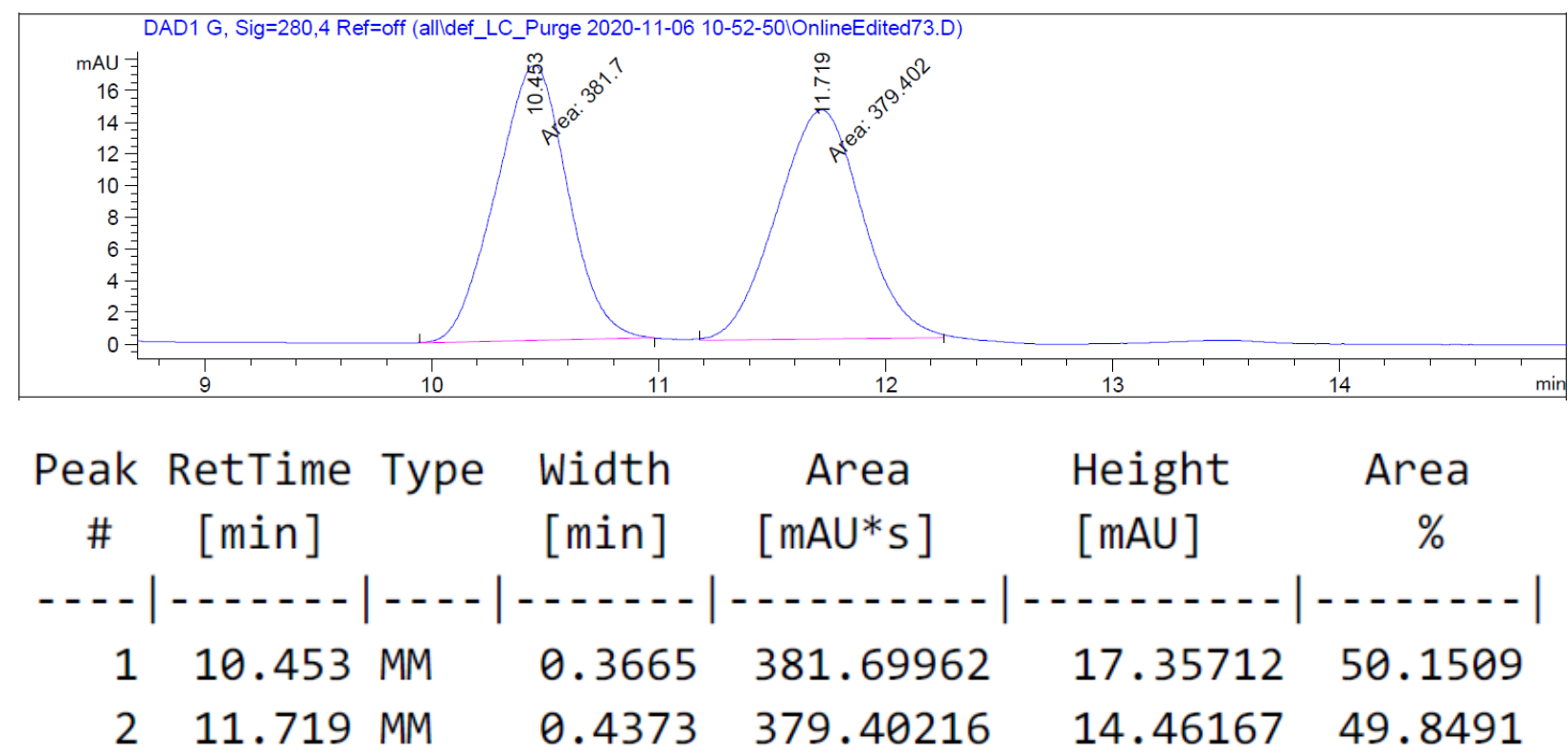



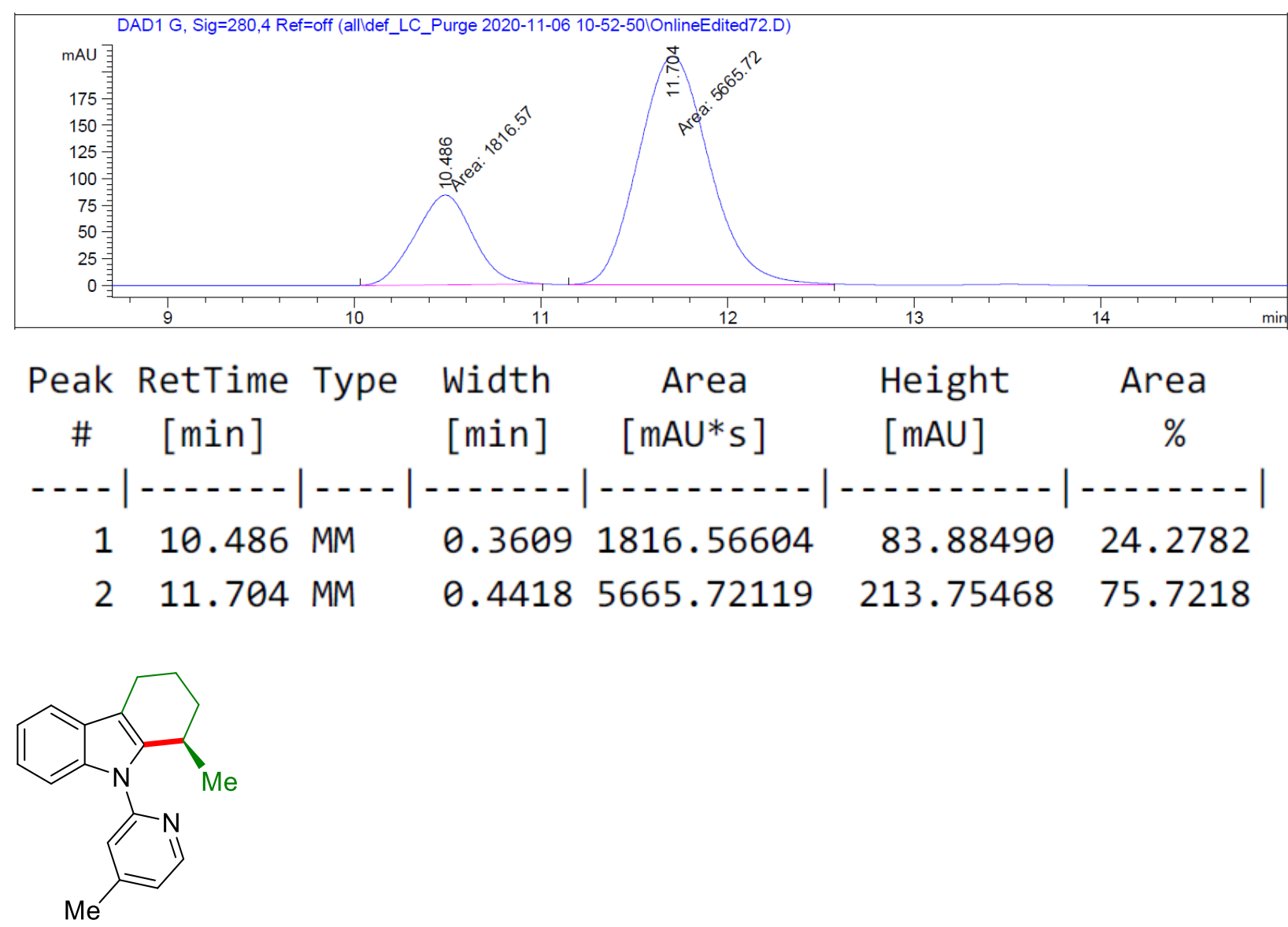

(R)-1-Methyl-9-(4-methylpyridin-2-yl)-2,3,4,9-tetrahydro-1H-carbazole (2a')): The general procedure A was followed using 1-(4-methylpyridin-2-yl)-3-(pent-4-en-1-yl)-1H-indole (1a'”) (69.0 mg, $0.25 \mathrm{mmol})$. Isolation by column chromatography ( $n$-hexane/EtOAc $=20: 1)$ yielded 2a', $(62.0 \mathrm{mg}, 90 \%)$ as a colourless oil. ${ }^{1} \mathrm{H}-\mathrm{NMR}\left(400 \mathrm{MHz}, \mathrm{CDCl}_{3}\right): \delta=8.47(\mathrm{~d}, J=5.1 \mathrm{~Hz}, 1 \mathrm{H})$, 7.53-7.46 (m, 1H), 7.40-7.34 (m, 1H), 7.27 (d, J=1.5 Hz, 1H), 7.16-7.09 (m, 3H), 3.59-3.45 (m, 1H), 2.86-2.67 (m, 2H), 2.47 (s, 3H), 2.17-2.05 (m, 1H), 1.98-1.80 (m, 2H), 1.68-1.58 (m, 1H), $0.84(\mathrm{~d}, J=6.9 \mathrm{~Hz}, 3 \mathrm{H}) .{ }^{13} \mathrm{C}-\mathrm{NMR}\left(100 \mathrm{MHz}, \mathrm{CDCl}_{3}\right): \delta=152.0\left(\mathrm{C}_{\mathrm{q}}\right), 149.9\left(\mathrm{C}_{\mathrm{q}}\right), 149.0(\mathrm{CH})$, $140.2\left(\mathrm{C}_{\mathrm{q}}\right), 136.9\left(\mathrm{C}_{\mathrm{q}}\right), 128.3\left(\mathrm{C}_{\mathrm{q}}\right), 122.6(\mathrm{CH}), 121.7(\mathrm{CH}), 120.9(\mathrm{CH}), 120.1(\mathrm{CH}), 118.1(\mathrm{CH})$, $112.5\left(\mathrm{C}_{\mathrm{q}}\right), 109.8(\mathrm{CH}), 31.8\left(\mathrm{CH}_{2}\right), 27.2(\mathrm{CH}), 21.5\left(\mathrm{CH}_{2}\right), 21.2\left(\mathrm{CH}_{3}\right), 20.3\left(\mathrm{CH}_{3}\right), 19.9\left(\mathrm{CH}_{2}\right)$. IR (ATR): 2930, 1601, 1478, 1454, 1224, 824, $736 \mathrm{~cm}^{-1}$. MS (ESI-TOF) $\mathrm{m} / z$ (relative intensity): 299 (10) $[\mathrm{M}+\mathrm{Na}]^{+}, 277(100)[\mathrm{M}+\mathrm{H}]^{+}$. HR-MS (ESI-TOF) $m / z:[\mathrm{M}+\mathrm{Na}]^{+}$Calcd. for $\mathrm{C}_{19} \mathrm{H}_{20} \mathrm{~N}_{2} \mathrm{Na}$ 299.1519; Found 299.1519. $[\alpha]_{\mathrm{D}}^{20}:-82.2\left(\mathrm{c}=1.0, \mathrm{CHCl}_{3}\right)$. HPLC separation $\left(\right.$ Chiralpak $^{\circledR}$ IB-3, $n$-hexane/EtOAc 95:5, $1.0 \mathrm{~mL} / \mathrm{min}$, detection at $273 \mathrm{~nm}$ ): $t_{r}$ (major) $=9.5 \mathrm{~min}$, $t_{r}($ minor $)=13.9 \min , 81: 19$ e.r. 

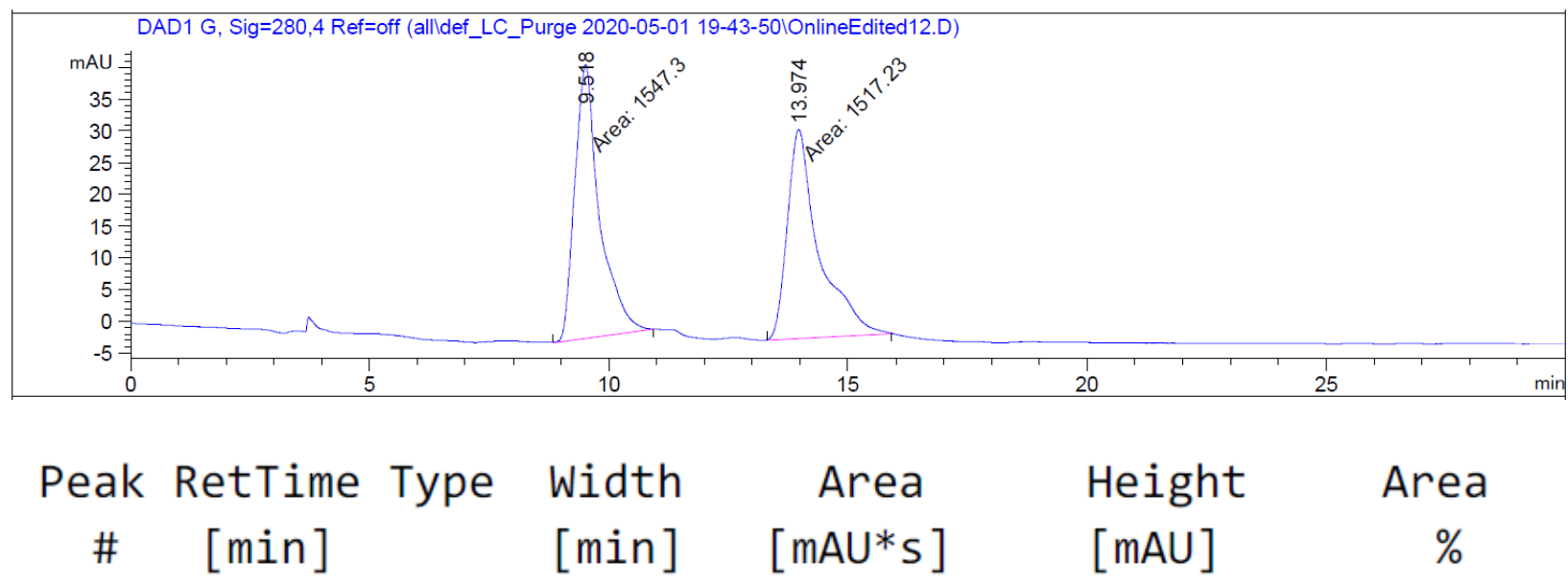
- - - | - - - - - | - - | - - - - | - - - - - - | - - - - - | - - - - | | $\begin{array}{llllll}1 & 9.518 \text { MM } & 0.5983 & 1547.30481 & 43.10374 & 50.4907\end{array}$ $\begin{array}{llllll}2 & 13.974 \text { MM } & 0.7677 & 1517.22803 & 32.93877 & 49.5093\end{array}$

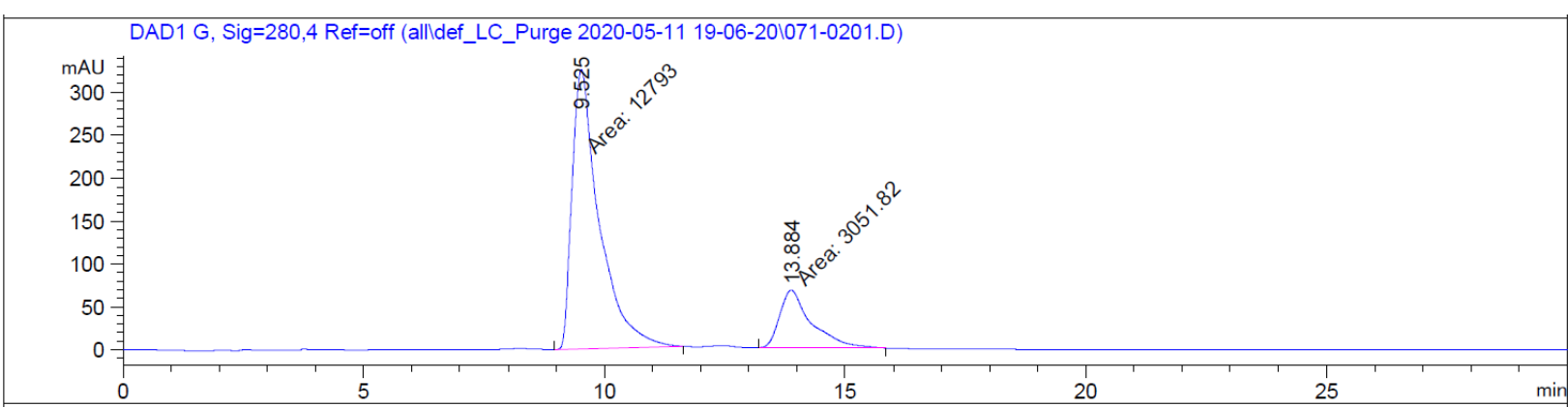

Peak RetTime Type Width Area Height Area $\begin{array}{lllll}\# & {[\mathrm{~min}]} & {[\mathrm{min}]} & {[\mathrm{mAU} * \mathrm{~s}]} & {[\mathrm{mAU}]}\end{array}$ - - - | - - - - - | - - - | - - - - | - - - - - - | - - - - - - | - - - - - |

$\begin{array}{llllll}1 & 9.525 \mathrm{MM} & 0.6555 & 1.27930 \mathrm{e} 4 & 325.26505 & 80.7393\end{array}$

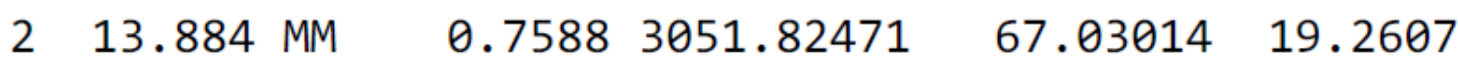<smiles>[M]C1CCCc2c1n(-c1ccc(C)cn1)c1ccccc21</smiles>

(R)-1-Methyl-9-(5-methylpyridin-2-yl)-2,3,4,9-tetrahydro-1H-carbazole (2a): The general procedure $\mathbf{A}$ was followed using 1-(5-methylpyridin-2-yl)-3-(pent-4-en-1-yl)-1 $H$-indole (1a) (69.0 mg, $0.25 \mathrm{mmol})$. Isolation by column chromatography $(n$-hexane/EtOAc $=20: 1)$ yielded $2 \mathbf{a}$ 
(65.7 mg, 95\%) as a colourless oil. ${ }^{1} \mathrm{H}-\mathrm{NMR}\left(400 \mathrm{MHz}, \mathrm{CDCl}_{3}\right): \delta=8.46(\mathrm{~d}, J=2.4,0.8 \mathrm{~Hz}, 1 \mathrm{H})$, 7.68 (ddd, $J=8.1,2.4,0.8 \mathrm{~Hz}, 1 \mathrm{H}), 7.54-7.47$ (m, 1H), 7.38-7.32 (m, 2H), 7.16-7.09 (m, 2H), $3.60-3.40(\mathrm{~m}, 1 \mathrm{H}), 2.86-2.66(\mathrm{~m}, 2 \mathrm{H}), 2.43$ (s, 3H), 2.17-2.05 (m, 1H), 2.00-1.80 (m, 2H), 1.71$1.59(\mathrm{~m}, 1 \mathrm{H}), 0.85(\mathrm{~d}, J=6.8 \mathrm{~Hz}, 3 \mathrm{H}) \cdot{ }^{13} \mathrm{C}-\mathrm{NMR}\left(100 \mathrm{MHz}, \mathrm{CDCl}_{3}\right): \delta=149.7(\mathrm{CH}), 149.7\left(\mathrm{C}_{\mathrm{q}}\right)$, 140.2 $\left(\mathrm{C}_{\mathrm{q}}\right), 138.8(\mathrm{CH}), 137.0\left(\mathrm{C}_{\mathrm{q}}\right), 131.1\left(\mathrm{C}_{\mathrm{q}}\right), 128.1\left(\mathrm{C}_{\mathrm{q}}\right), 121.7(\mathrm{CH}), 120.0(\mathrm{CH}), 119.8(\mathrm{CH})$, $118.0(\mathrm{CH}), 112.1\left(\mathrm{C}_{\mathrm{q}}\right), 109.7(\mathrm{CH}), 31.8\left(\mathrm{CH}_{2}\right), 27.1(\mathrm{CH}), 21.5\left(\mathrm{CH}_{2}\right), 20.3\left(\mathrm{CH}_{3}\right), 19.9\left(\mathrm{CH}_{2}\right)$, $18.0\left(\mathrm{CH}_{3}\right)$. IR (ATR): 2928, 1570, 1483, 1385, 1224, 1028, 833, $737 \mathrm{~cm}^{-1}$. MS (ESI-TOF) $\mathrm{m} / \mathrm{z}$ (relative intensity): $299(10)[\mathrm{M}+\mathrm{Na}]^{+}, 277(100)[\mathrm{M}+\mathrm{H}]^{+}$. HR-MS (ESI-TOF) $\mathrm{m} / \mathrm{z}:[\mathrm{M}+\mathrm{H}]^{+}$ Calcd. for $\mathrm{C}_{19} \mathrm{H}_{21} \mathrm{~N}_{2}$ 277.1699; Found 277.1700. $[\alpha]_{\mathrm{D}}^{20}:-95.3\left(\mathrm{c}=1.0, \mathrm{CHCl}_{3}\right)$. HPLC separation (Chiralpak ${ }^{\circledR} \mathrm{ID}-3, n$-hexane/EtOAc $90: 10,1.0 \mathrm{~mL} / \mathrm{min}$, detection at $\left.273 \mathrm{~nm}\right): t_{r}$ (major) $=7.5 \mathrm{~min}$, $t_{r}($ minor $)=5.8 \mathrm{~min}, 85: 15$ e.r.
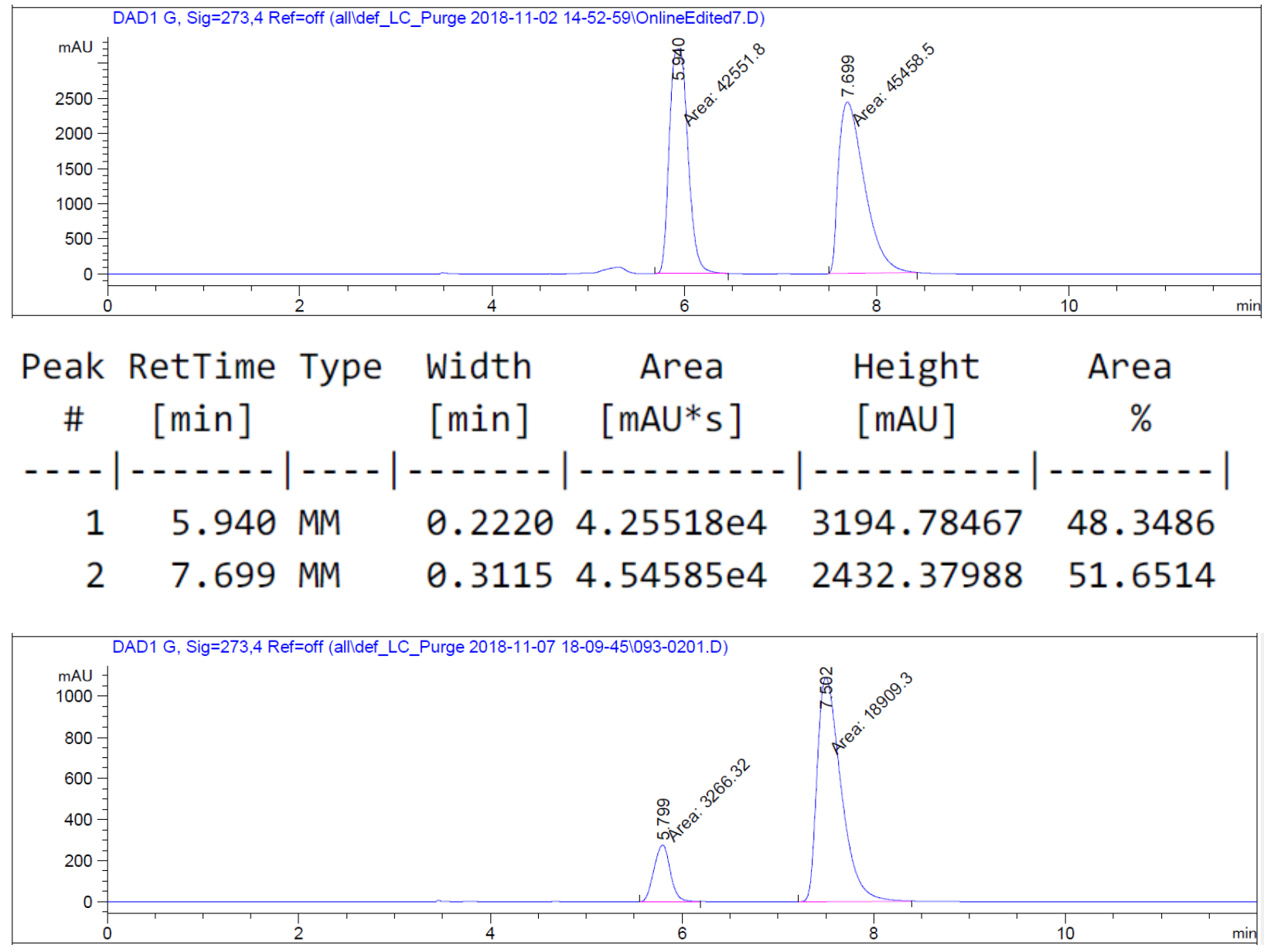

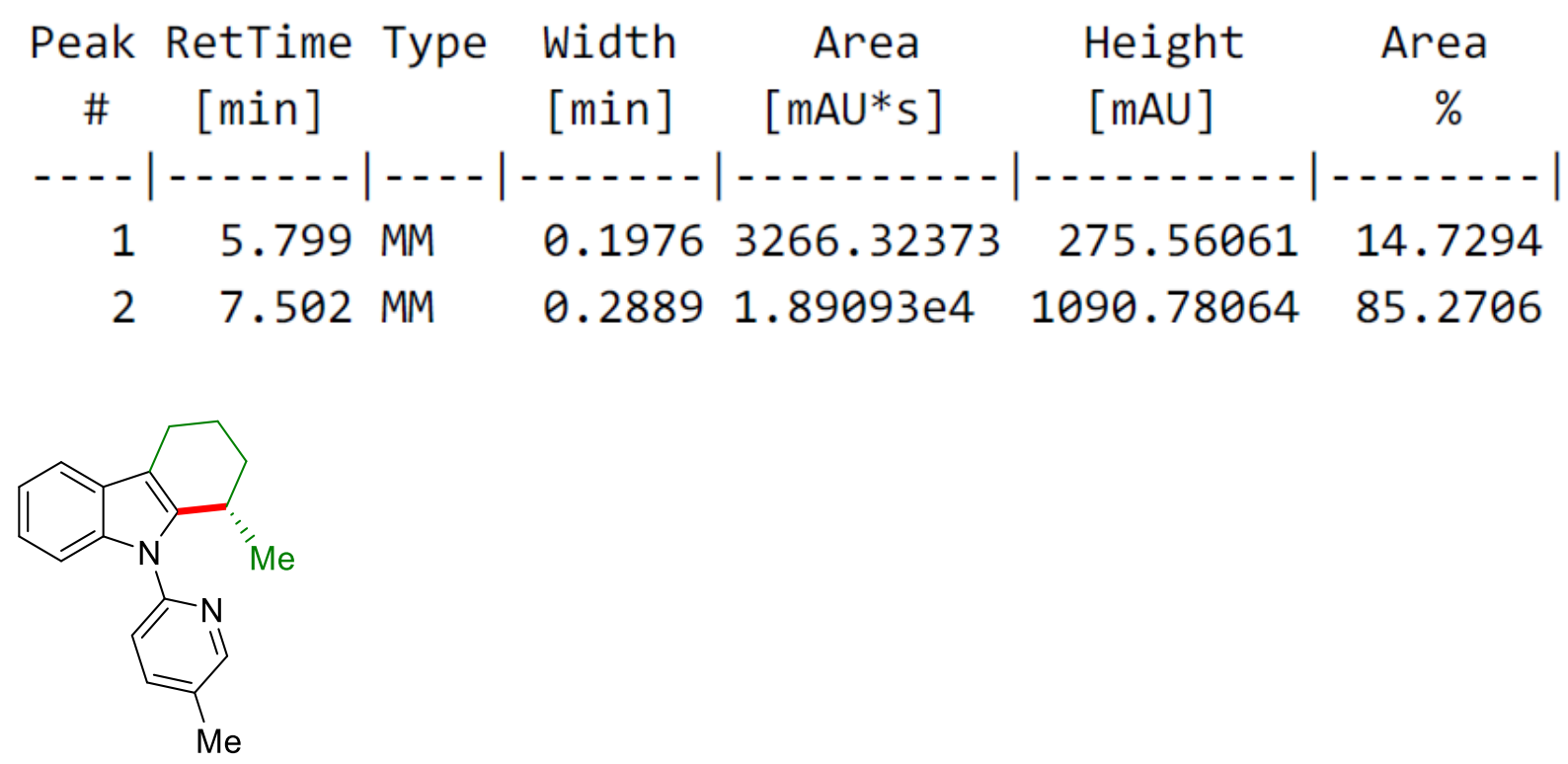

(S)-1-Methyl-9-(5-methylpyridin-2-yl)-2,3,4,9-tetrahydro-1H-carbazole (2a): A modifed general procedure A was followed using 1-(5-methylpyridin-2-yl)-3-(pent-4-en-1-yl)-1H-indole (1a) $(69.0 \mathrm{mg}, 0.25 \mathrm{mmol})$ with ent-CA8. Isolation by column chromatography ( $n$-hexane/EtOAc $=20: 1)$ yielded ent-2a $(64.0 \mathrm{mg}, 94 \%)$ as a colourless oil. $[\alpha]_{\mathrm{D}}^{20}:+94.4\left(\mathrm{c}=1.0, \mathrm{CHCl}_{3}\right)$. HPLC separation (Chiralpak ${ }^{\circledR}$ ID-3, $n$-hexane/EtOAc 90:10, $1.0 \mathrm{~mL} / \mathrm{min}$, detection at $273 \mathrm{~nm}$ ): $t_{r}$ (major) $=5.9 \min , t_{r}($ minor $)=7.5 \mathrm{~min}, 15: 85$ e.r.

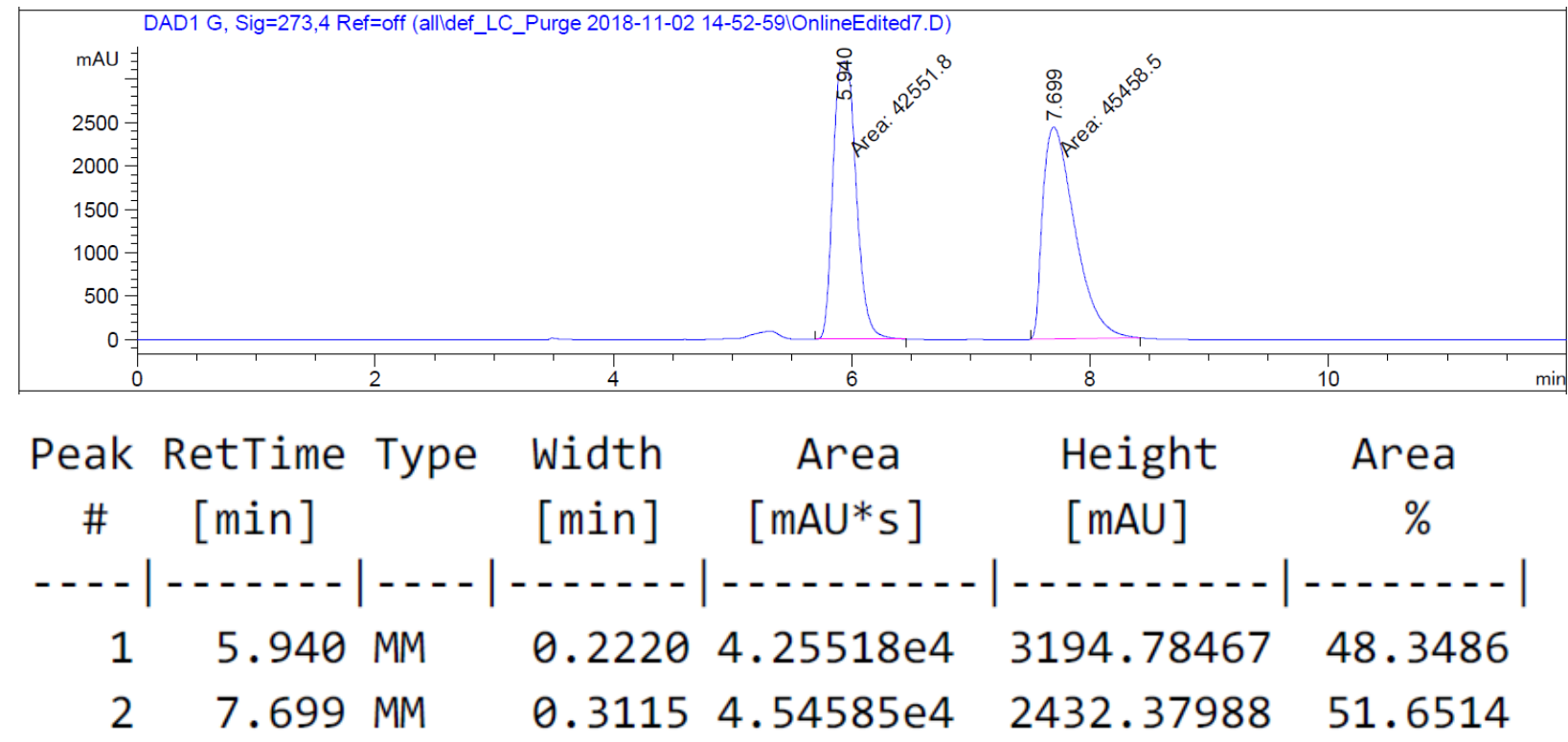




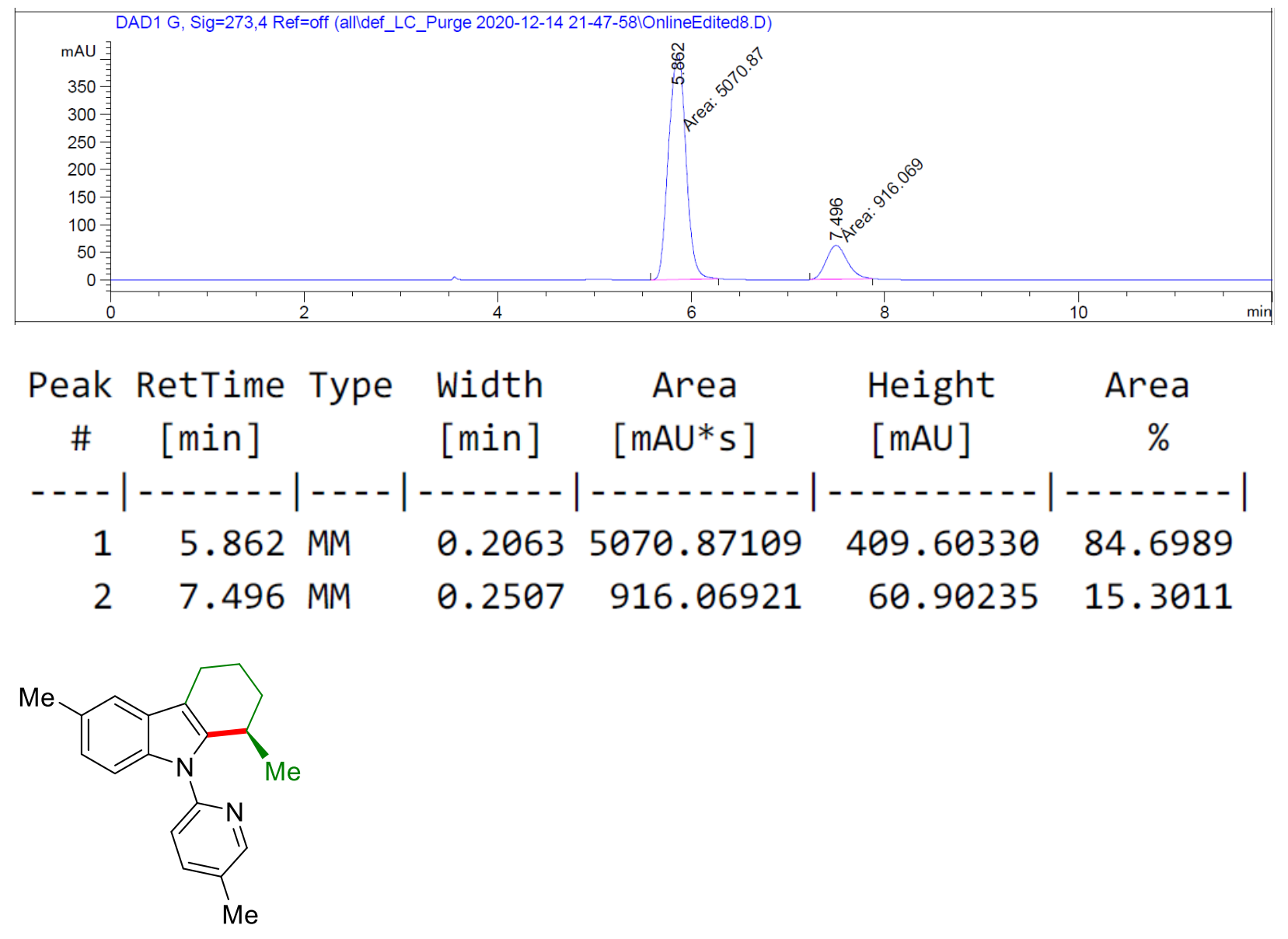

(R)-1,6-Dimethyl-9-(5-methylpyridin-2-yl)-2,3,4,9-tetrahydro-1H-carbazole $\quad(2 \mathrm{~b}): \quad$ The general procedure A was followed using 5-methyl-1-(5-methylpyridin-2-yl)-3-(pent-4-en-1-yl)$1 H$-indole $(\mathbf{1 b})(72.6 \mathrm{mg}, 0.25 \mathrm{mmol})$. Isolation by column chromatography $(n$-hexane/EtOAc $=$ 10:1) yielded $\mathbf{2 b}(60.3 \mathrm{mg}, 83 \%)$ as a yellow oil. ${ }^{1} \mathrm{H}-\mathrm{NMR}\left(300 \mathrm{MHz}, \mathrm{CDCl}_{3}\right): 8.47$ (d, $J=2.4 \mathrm{~Hz}$, $1 \mathrm{H}), 7.69(\mathrm{dd}, J=8.1,2.4 \mathrm{~Hz}, 1 \mathrm{H}), 7.36(\mathrm{~d}, J=8.1 \mathrm{~Hz}, 1 \mathrm{H}), 7.33(\mathrm{~s}, 1 \mathrm{H}), 7.28(\mathrm{~d}, J=8.3 \mathrm{~Hz}, 1 \mathrm{H})$, $6.98(\mathrm{~d}, J=8.3 \mathrm{~Hz}, 1 \mathrm{H}), 3.58-3.47(\mathrm{~m}, 1 \mathrm{H}), 2.85-2.68(\mathrm{~m}, 2 \mathrm{H}), 2.49$ (s, 3H), 2.45 (s, 3H), 2.20$2.06(\mathrm{~m}, 1 \mathrm{H}), 1.99-1.85(\mathrm{~m}, 2 \mathrm{H}), 1.72-1.61(\mathrm{~m}, 1 \mathrm{H}), 0.87(\mathrm{~d}, J=6.8 \mathrm{~Hz}, 3 \mathrm{H}) .{ }^{13} \mathrm{C}-\mathrm{NMR}(100$ $\left.\mathrm{MHz}, \mathrm{CDCl}_{3}\right): \delta=150.0\left(\mathrm{C}_{\mathrm{q}}\right), 149.7(\mathrm{CH}), 140.4\left(\mathrm{C}_{\mathrm{q}}\right), 138.9(\mathrm{CH}), 135.4\left(\mathrm{C}_{\mathrm{q}}\right), 131.0\left(\mathrm{C}_{\mathrm{q}}\right), 129.4$ $\left(\mathrm{C}_{\mathrm{q}}\right), 128.5\left(\mathrm{C}_{\mathrm{q}}\right), 123.2(\mathrm{CH}), 119.7(\mathrm{CH}), 118.0(\mathrm{CH}), 111.9\left(\mathrm{C}_{\mathrm{q}}\right), 109.6(\mathrm{CH}), 31.9\left(\mathrm{CH}_{2}\right), 27.3$ $(\mathrm{CH}), 21.6\left(\mathrm{CH}_{2}\right), 21.5\left(\mathrm{CH}_{3}\right), 20.5\left(\mathrm{CH}_{3}\right), 20.1\left(\mathrm{CH}_{2}\right), 18.2\left(\mathrm{CH}_{3}\right)$. IR (ATR): 1597, 1571, 1482, 1405, 1384, 1295, 1224, 1027, 793, $747 \mathrm{~cm}^{-1}$. MS (ESI-TOF) $\mathrm{m} / z$ (relative intensity): 291 (100) $[\mathrm{M}+\mathrm{H}]^{+} \mathrm{HR}-\mathrm{MS}$ (ESI-TOF) $\mathrm{m} / z:[\mathrm{M}+\mathrm{H}]^{+}$Calcd. for $\mathrm{C}_{20} \mathrm{H}_{23} \mathrm{~N}_{2}$ 291.1861; Found: 291.1856 . $[\alpha]_{\mathrm{D}}^{20}:-37.9\left(\mathrm{c}=1.0, \mathrm{CHCl}_{3}\right)$. HPLC separation $\left(\right.$ Chiralpak $^{\circledR}$ ID-3, $n$-hexane/EtOAc 90:10, 1.0 $\mathrm{mL} / \mathrm{min}$, detection at $273 \mathrm{~nm}): t_{r}($ major $)=8.0 \mathrm{~min}, t_{r}($ minor $)=5.4 \mathrm{~min}, 84: 16$ e.r. 

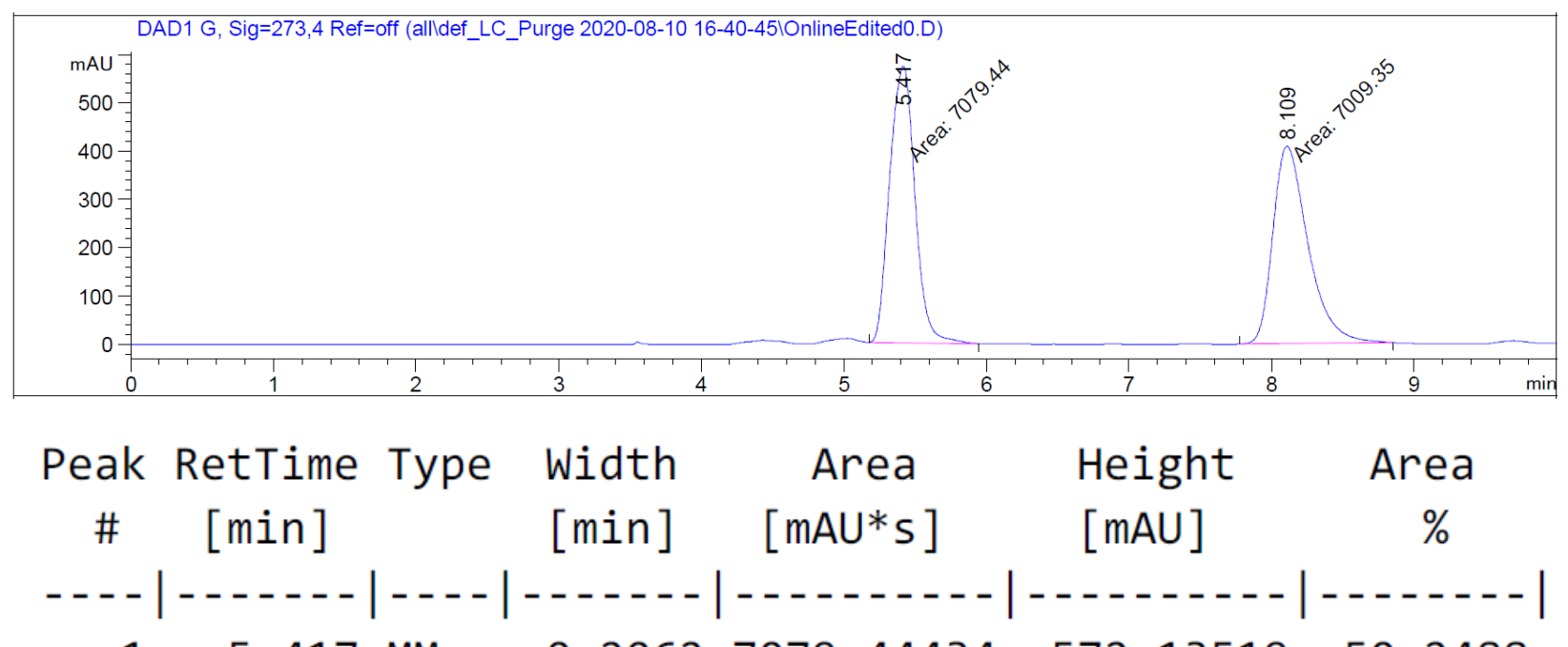

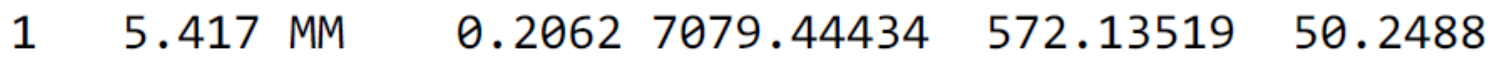

$\begin{array}{llllll}2 & 8.109 \text { MM } & 0.2858 & 7009.34814 & 408.70639 & 49.7512\end{array}$
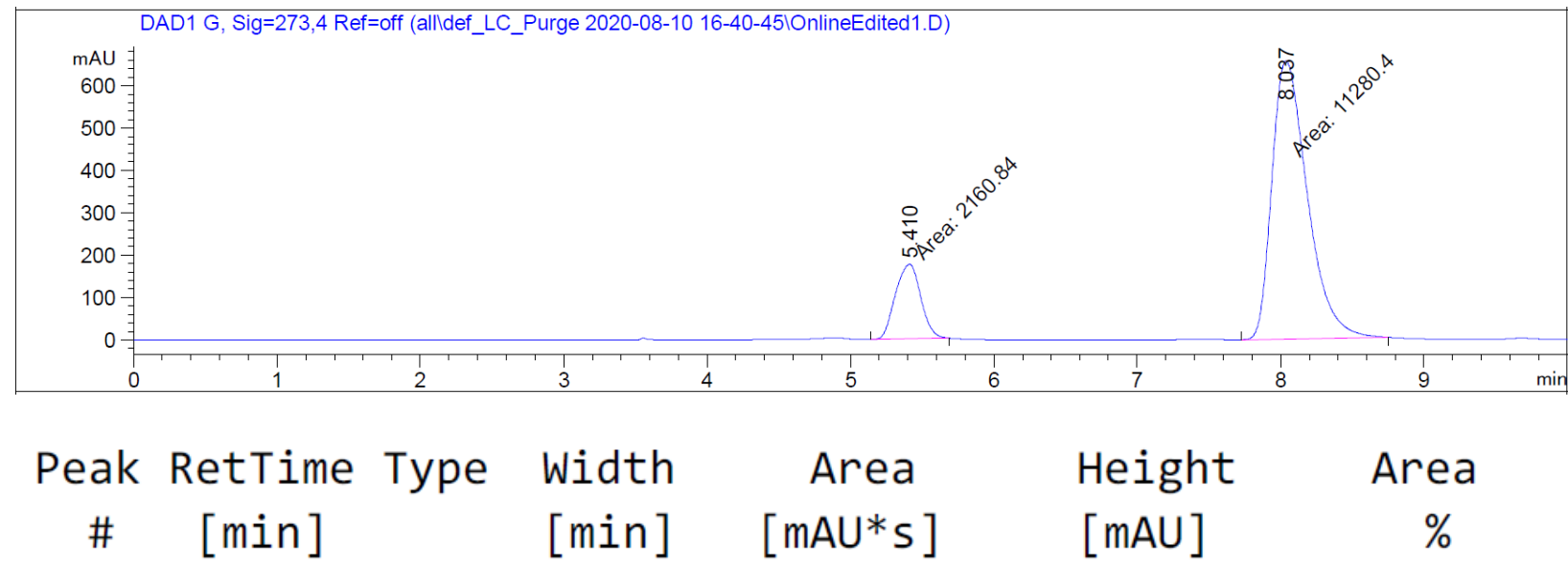

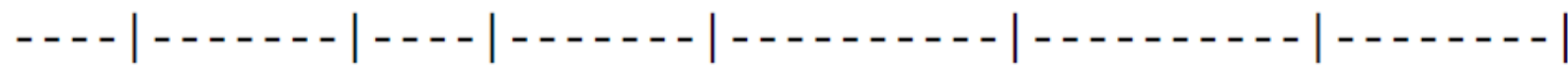

$\begin{array}{llllll}1 & 5.410 \mathrm{MM} & 0.2048 & 2160.83691 & 175.85220 & 16.0762\end{array}$

$\begin{array}{lllllll}2 & 8.037 & \text { MM } & 0.2863 & 1.12804 \mathrm{e} 4 & 656.75031 & 83.9238\end{array}$<smiles>Cc1ccc(-n2c3c(c4ccc(C)cc42)CCCC3C)nc1</smiles>

(R)-1,7-Dimethyl-9-(5-methylpyridin-2-yl)-2,3,4,9-tetrahydro-1H-carbazole (2c): The general procedure A was followed using 6-methyl-1-(5-methylpyridin-2-yl)-3-(pent-4-en-1-yl)-1H-indole (1c) $(72.6 \mathrm{mg}, 0.25 \mathrm{mmol})$. Isolation by column chromatography ( $n$-hexane/EtOAc $=20: 1)$ yielded 
2c $(68.4 \mathrm{mg}, 94 \%)$ as a yellow solid. M.p.: 92-94 ${ }^{\circ} \mathrm{C} .{ }^{1} \mathrm{H}-\mathrm{NMR}\left(400 \mathrm{MHz}, \mathrm{CDCl}_{3}\right): \delta=8.45$ (dd, $J=2.4,0.8 \mathrm{~Hz}, 1 \mathrm{H}), 7.74-7.62(\mathrm{~m}, 1 \mathrm{H}), 7.38(\mathrm{~d}, J=7.9 \mathrm{~Hz}, 1 \mathrm{H}), 7.33(\mathrm{~d}, J=7.9 \mathrm{~Hz}, 1 \mathrm{H}), 7.15$ $(\mathrm{dd}, J=1.4,0.8 \mathrm{~Hz}, 1 \mathrm{H}), 6.96(\mathrm{ddd}, J=7.9,1.4,0.8 \mathrm{~Hz}, 1 \mathrm{H}), 3.50-3.39$ (m, 1H), 2.81-2.64 (m, 2H), $2.43(\mathrm{~s}, 3 \mathrm{H}), 2.41(\mathrm{~s}, 3 \mathrm{H}), 2.16-2.04(\mathrm{~m}, 1 \mathrm{H}), 1.98-1.80(\mathrm{~m}, 2 \mathrm{H}), 1.68-1.58(\mathrm{~m}, 1 \mathrm{H}), 0.83(\mathrm{~d}$, $J=6.8 \mathrm{~Hz}, 3 \mathrm{H}) .{ }^{13} \mathrm{C}-\mathrm{NMR}\left(100 \mathrm{MHz}, \mathrm{CDCl}_{3}\right): \delta=149.8\left(\mathrm{C}_{\mathrm{q}}\right), 149.7(\mathrm{CH}), 139.4\left(\mathrm{C}_{\mathrm{q}}\right), 138.8(\mathrm{CH})$, 137.4 $\left(\mathrm{C}_{\mathrm{q}}\right), 131.4\left(\mathrm{C}_{\mathrm{q}}\right), 131.0\left(\mathrm{C}_{\mathrm{q}}\right), 125.9\left(\mathrm{C}_{\mathrm{q}}\right), 121.5(\mathrm{CH}), 119.9(\mathrm{CH}), 117.6(\mathrm{CH}), 112.0\left(\mathrm{C}_{\mathrm{q}}\right)$, $109.9(\mathrm{CH}), 31.8\left(\mathrm{CH}_{2}\right), 27.1(\mathrm{CH}), 21.8\left(\mathrm{CH}_{3}\right), 21.5\left(\mathrm{CH}_{2}\right), 20.3\left(\mathrm{CH}_{3}\right), 19.9\left(\mathrm{CH}_{2}\right), 18.0\left(\mathrm{CH}_{3}\right)$. IR (ATR): 2927, 2858, 1597, 1438, 1388, 1224, 1171, 1029, $841 \mathrm{~cm}^{-1}$. MS (ESI-TOF) $\mathrm{m} / \mathrm{z}$ (relative intensity): $603(5)[2 \mathrm{M}+\mathrm{Na}]^{+}, 581(15)[2 \mathrm{M}+\mathrm{H}]^{+}, 313(10)[\mathrm{M}+\mathrm{Na}]^{+}, 291(100)[\mathrm{M}+\mathrm{H}]^{+}$. HRMS (ESI-TOF) $m / z:[\mathrm{M}+\mathrm{H}]^{+}$Calcd. for $\mathrm{C}_{20} \mathrm{H}_{23} \mathrm{~N}_{2} 291.1856$ found 291.1859. $[\alpha]_{\mathrm{D}}^{20}:-79.9(\mathrm{c}=$ 1.0, $\mathrm{CHCl}_{3}$ ). HPLC separation (Chiralpak ${ }^{\circledR}$ ID-3, $n$-hexane/EtOAc 90:10, $1.0 \mathrm{~mL} / \mathrm{min}$, detection at $273 \mathrm{~nm}): t_{r}$ (major) $=7.2 \mathrm{~min}, t_{r}($ minor $)=6.1 \mathrm{~min}, 82: 18$ e.r.

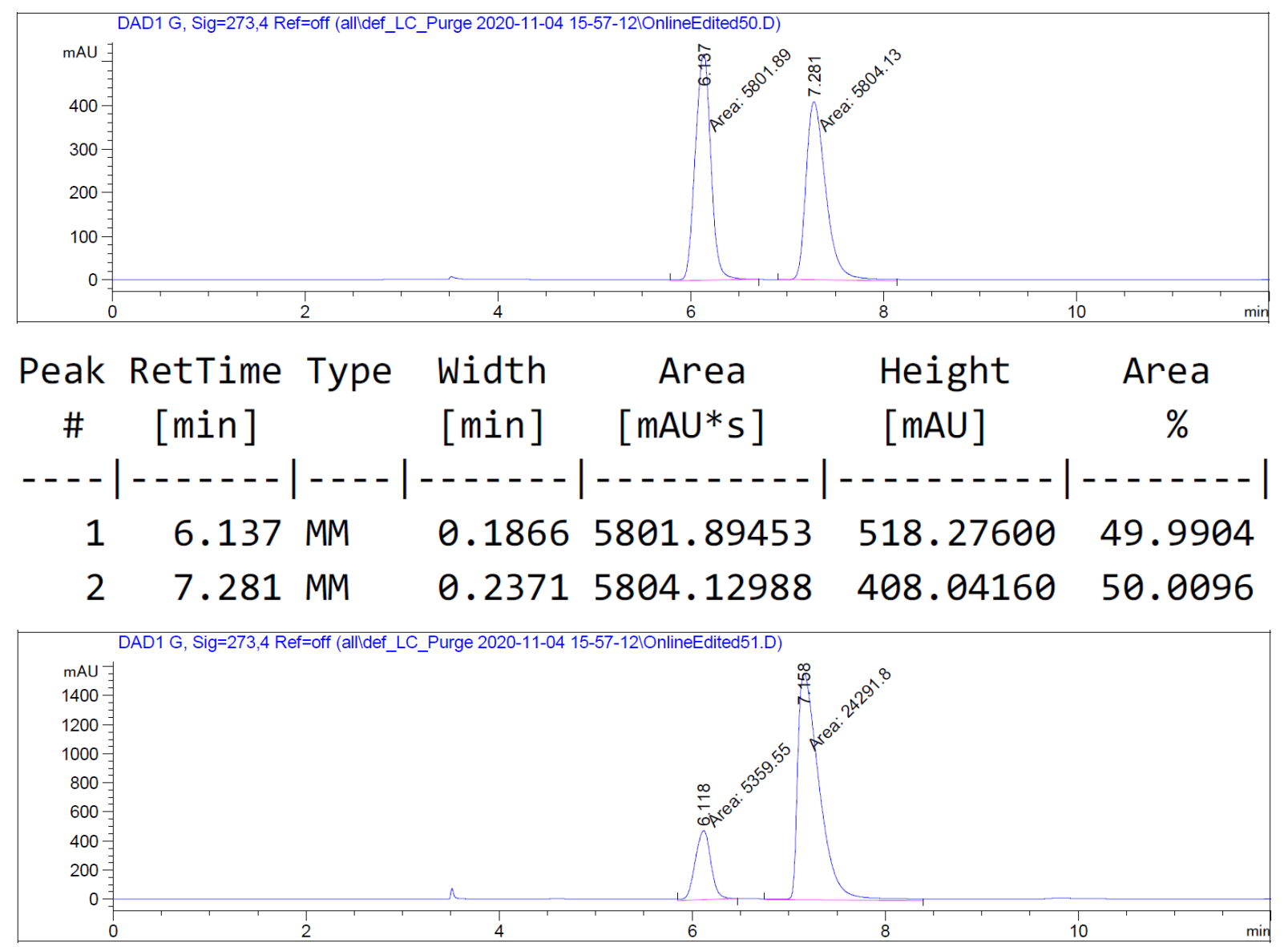



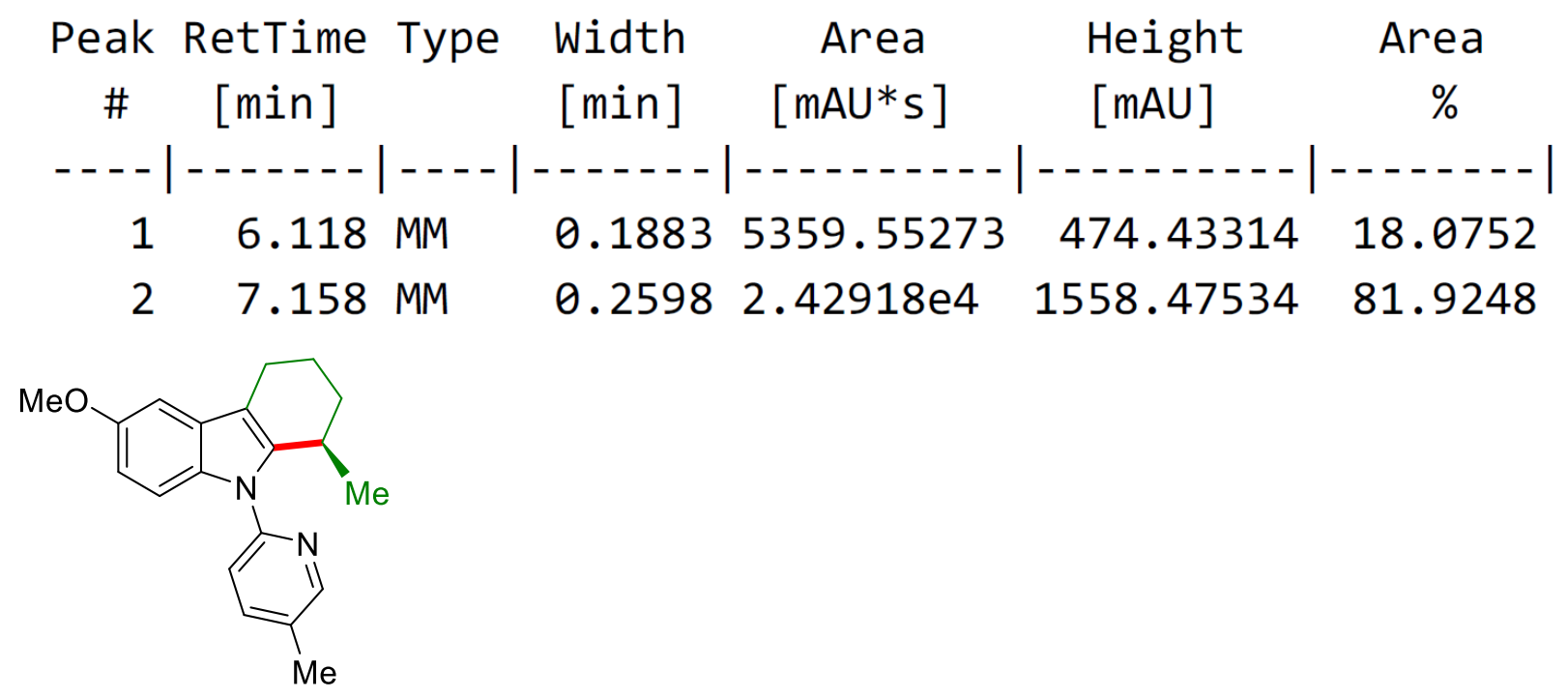

(R)-6-Methoxy-1-methyl-9-(5-methylpyridin-2-yl)-2,3,4,9-tetrahydro-1H-carbazole (2d):

The general procedure A was followed using 5-methoxy-1-(5-methylpyridin-2-yl)-3-(pent-4-en1-yl)-1H-indole (1d) $\quad(76.5 \mathrm{mg}, 0.25 \mathrm{mmol})$. Isolation by column chromatography $(n-$ hexane/EtOAc $=10: 1)$ yielded $2 d(68.0 \mathrm{mg}, 89 \%)$ as a yellow oil. ${ }^{1} \mathrm{H}-\mathrm{NMR}\left(400 \mathrm{MHz}, \mathrm{CDCl}_{3}\right): \delta$ $=8.43(\mathrm{~d}, J=2.3 \mathrm{~Hz}, 1 \mathrm{H}), 7.65(\mathrm{dd}, J=8.1,2.3 \mathrm{~Hz}, 1 \mathrm{H}), 7.31(\mathrm{~d}, J=8.1 \mathrm{~Hz}, 1 \mathrm{H}), 7.26(\mathrm{~d}, J=8.8$ $\mathrm{Hz}, 1 \mathrm{H}), 6.96(\mathrm{~d}, J=2.5 \mathrm{~Hz}, 1 \mathrm{H}), 6.77(\mathrm{dd}, J=8.8,2.5 \mathrm{~Hz}, 1 \mathrm{H}), 3.87$ (s, 3H), 3.55-3.40 (m, 1H), 2.80-2.64 (m, 2H), 2.41 (s, 3H), 2.20-2.02 (m, 1H), 2.01-1.80 (m, 2H), 1.68-1.56 (m, 1H), 0.84 $(\mathrm{d}, J=6.9 \mathrm{~Hz}, 3 \mathrm{H}) .{ }^{13} \mathrm{C}-\mathrm{NMR}\left(100 \mathrm{MHz}, \mathrm{CDCl}_{3}\right): \delta=154.5\left(\mathrm{C}_{\mathrm{q}}\right), 149.8\left(\mathrm{C}_{\mathrm{q}}\right), 149.6(\mathrm{CH}), 140.9$ $\left(\mathrm{C}_{\mathrm{q}}\right), 138.8(\mathrm{CH}), 132.1\left(\mathrm{C}_{\mathrm{q}}\right), 130.9\left(\mathrm{C}_{\mathrm{q}}\right), 128.6\left(\mathrm{C}_{\mathrm{q}}\right), 119.5(\mathrm{CH}), 111.9\left(\mathrm{C}_{\mathrm{q}}\right), 111.0(\mathrm{CH}), 110.6$ $(\mathrm{CH}), 100.6(\mathrm{CH}), 56.0\left(\mathrm{CH}_{3}\right), 31.8\left(\mathrm{CH}_{2}\right), 27.2(\mathrm{CH}), 21.5\left(\mathrm{CH}_{2}\right), 20.3\left(\mathrm{CH}_{3}\right), 19.9\left(\mathrm{CH}_{2}\right), 18.0$ $\left(\mathrm{CH}_{3}\right)$. IR (ATR): 2930, 1596, 1483, 1440, 1388, 1156, $820 \mathrm{~cm}^{-1}$. MS (ESI-TOF) $\mathrm{m} / \mathrm{z}$ (relative intensity): 329 (25) $[\mathrm{M}+\mathrm{Na}]^{+}, 307(100)[\mathrm{M}+\mathrm{H}]^{+}$. HR-MS (ESI-TOF) $m / z:[\mathrm{M}+\mathrm{H}]^{+} \mathrm{Calcd}$. for $\mathrm{C}_{20} \mathrm{H}_{23} \mathrm{~N}_{2} \mathrm{O}$ 307.1805; Found 307.1808. [ $\left.\alpha\right]_{\mathrm{D}}^{20}$ : -51.6 $\left(\mathrm{c}=1.0, \mathrm{CHCl}_{3}\right)$. HPLC separation $\left(\right.$ Chiralpak $^{\circledR} \mathrm{ID}-3, n$-hexane/EtOAc $90: 10,1.0 \mathrm{~mL} / \mathrm{min}$, detection at $\left.273 \mathrm{~nm}\right): t_{r}$ (major) $=13.6 \mathrm{~min}$, $t_{r}($ minor $)=8.6 \mathrm{~min}, 85: 15$ e.r.

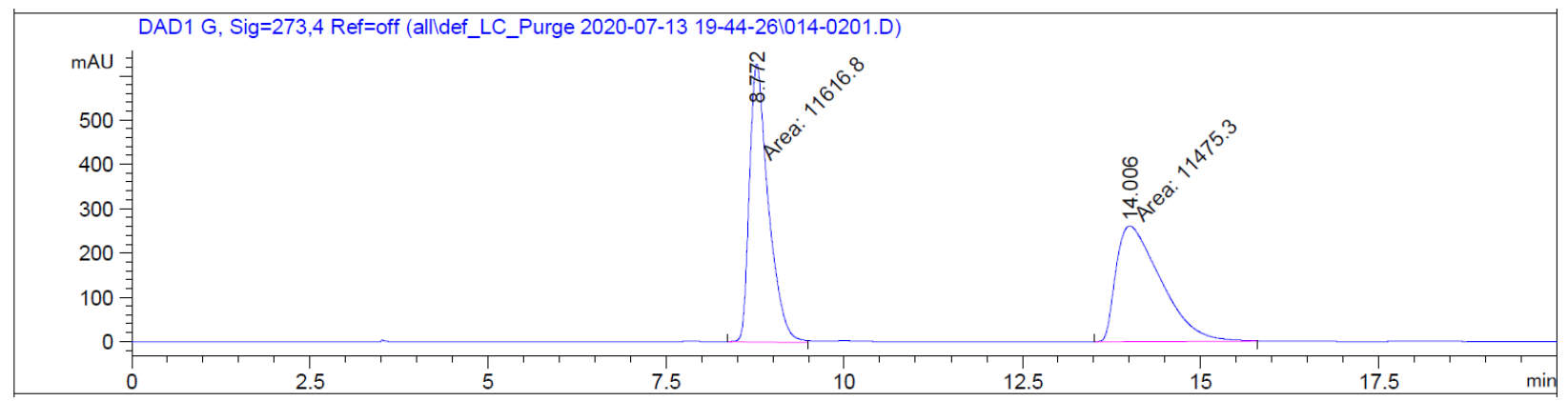



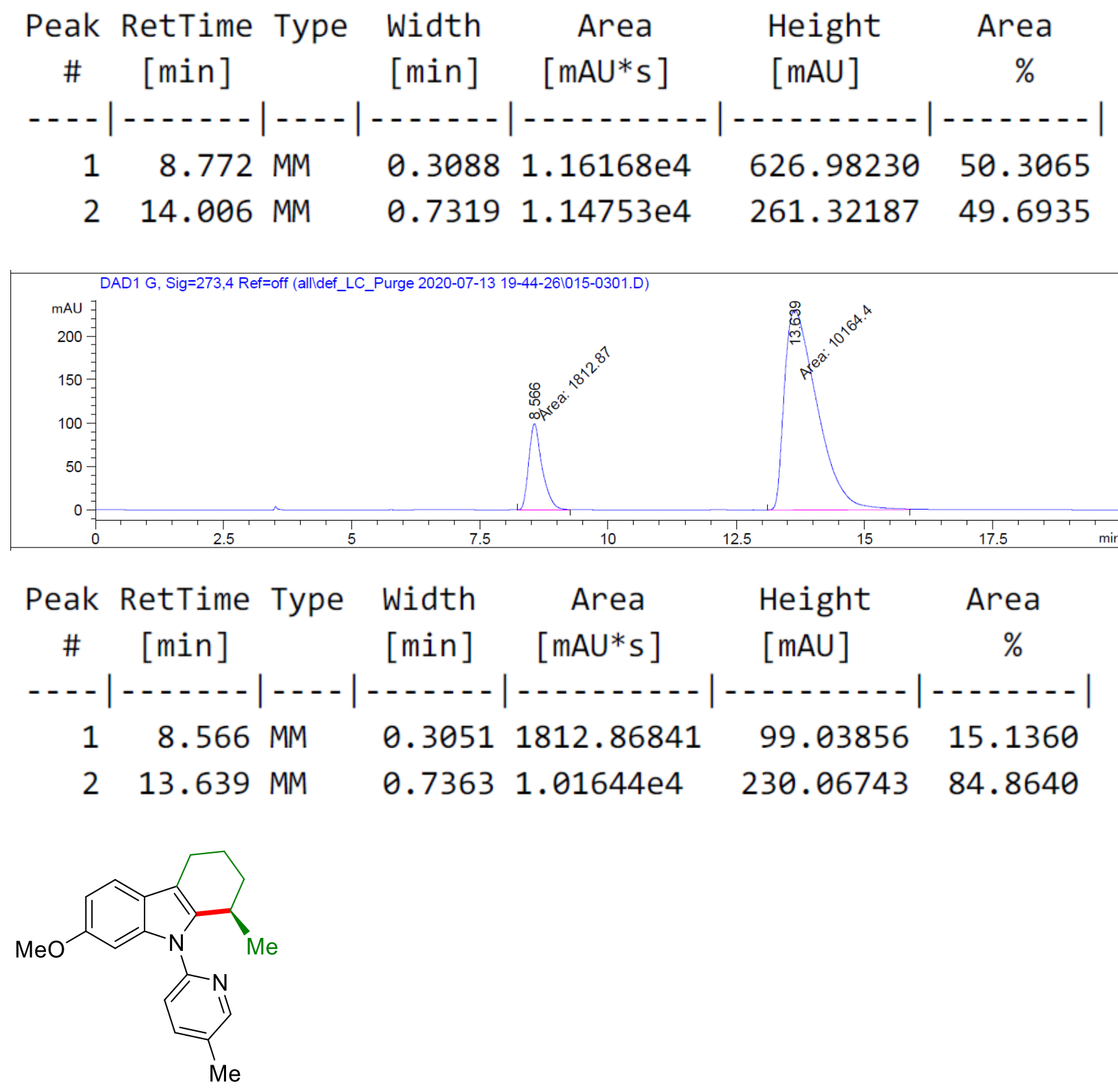

(R)-7-Methoxy-1-methyl-9-(5-methylpyridin-2-yl)-2,3,4,9-tetrahydro-1H-carbazole (2e):

The general procedure A was followed using 6-methoxy-1-(5-methylpyridin-2-yl)-3-(pent-4-en1-yl)-1H-indole (1e) $\quad(76.5 \mathrm{mg}, 0.25 \mathrm{mmol})$. Isolation by column chromatography $(n$ hexane/EtOAc $=10: 1)$ yielded $2 \mathbf{e}(72.0 \mathrm{mg}, 95 \%)$ as a yellow oil. ${ }^{1} \mathrm{H}-\mathrm{NMR}\left(300 \mathrm{MHz}, \mathrm{CDCl}_{3}\right): \delta$ $=8.46(\mathrm{~d}, J=2.4 \mathrm{~Hz}, 1 \mathrm{H}), 7.67(\mathrm{dd}, J=8.1,2.4 \mathrm{~Hz}, 1 \mathrm{H}), 7.37(\mathrm{~d}, J=8.5 \mathrm{~Hz}, 1 \mathrm{H}), 7.32(\mathrm{~d}, J=8.1$ Hz, 1H), 6.90 (d, J=2.3 Hz, 1H), 6.79 (dd, J=8.5, 2.3 Hz, 1H), 3.79 (s, 3H), 3.46-3.34 (m, 1H), 2.83-2.60 (m, 2H), 2.43 (s, 3H), 2.15-2.00 (m, 1H), 1.95-1.80 (m, 2H), 1.69-1.56 (m, 1H), 0.82 $(\mathrm{d}, J=6.8 \mathrm{~Hz}, 3 \mathrm{H}) .{ }^{13} \mathrm{C}-\mathrm{NMR}\left(100 \mathrm{MHz}, \mathrm{CDCl}_{3}\right): \delta=156.4\left(\mathrm{C}_{\mathrm{q}}\right), 149.8\left(\mathrm{C}_{\mathrm{q}}\right), 149.8(\mathrm{CH}), 139.0$ $\left(\mathrm{C}_{\mathrm{q}}\right), 138.9(\mathrm{CH}), 137.8\left(\mathrm{C}_{\mathrm{q}}\right), 131.1\left(\mathrm{C}_{\mathrm{q}}\right), 122.6\left(\mathrm{C}_{\mathrm{q}}\right), 119.7(\mathrm{CH}), 118.5(\mathrm{CH}), 112.1\left(\mathrm{C}_{\mathrm{q}}\right), 109.0$ 
$(\mathrm{CH}), 94.7(\mathrm{CH}), 55.9\left(\mathrm{CH}_{3}\right), 31.8\left(\mathrm{CH}_{2}\right), 27.2(\mathrm{CH}), 21.5\left(\mathrm{CH}_{2}\right), 20.4\left(\mathrm{CH}_{3}\right), 20.0\left(\mathrm{CH}_{2}\right), 18.1$ $\left(\mathrm{CH}_{3}\right)$. IR (ATR): 2930, 1596, 1475, 1406, 1227, 1203, 1025, $796 \mathrm{~cm}^{-1}$. MS (ESI) $\mathrm{m} / z$ (relative intensity): 329 (10) $[\mathrm{M}+\mathrm{Na}]^{+}, 307(100)[\mathrm{M}+\mathrm{H}]^{+}$. HR-MS (ESI-TOF) $\mathrm{m} / z:[\mathrm{M}+\mathrm{Na}]^{+} \mathrm{Calcd}$. for $\mathrm{C}_{20} \mathrm{H}_{22} \mathrm{~N}_{2} \mathrm{ONa}$ 329.1624; Found 329.1622. $[\alpha]_{\mathrm{D}}^{20}:-65.1\left(\mathrm{c}=1.0, \mathrm{CHCl}_{3}\right)$. HPLC separation $\left(\right.$ Chiralpak $^{\circledR}$ ID-3, $n$-hexane/EtOAc 90:10, $1.0 \mathrm{~mL} / \mathrm{min}$, detection at $\left.273 \mathrm{~nm}\right): t_{r}$ (major) $=13.8 \mathrm{~min}$, $t_{r}($ minor $)=9.6 \mathrm{~min}, 84: 16$ e.r.

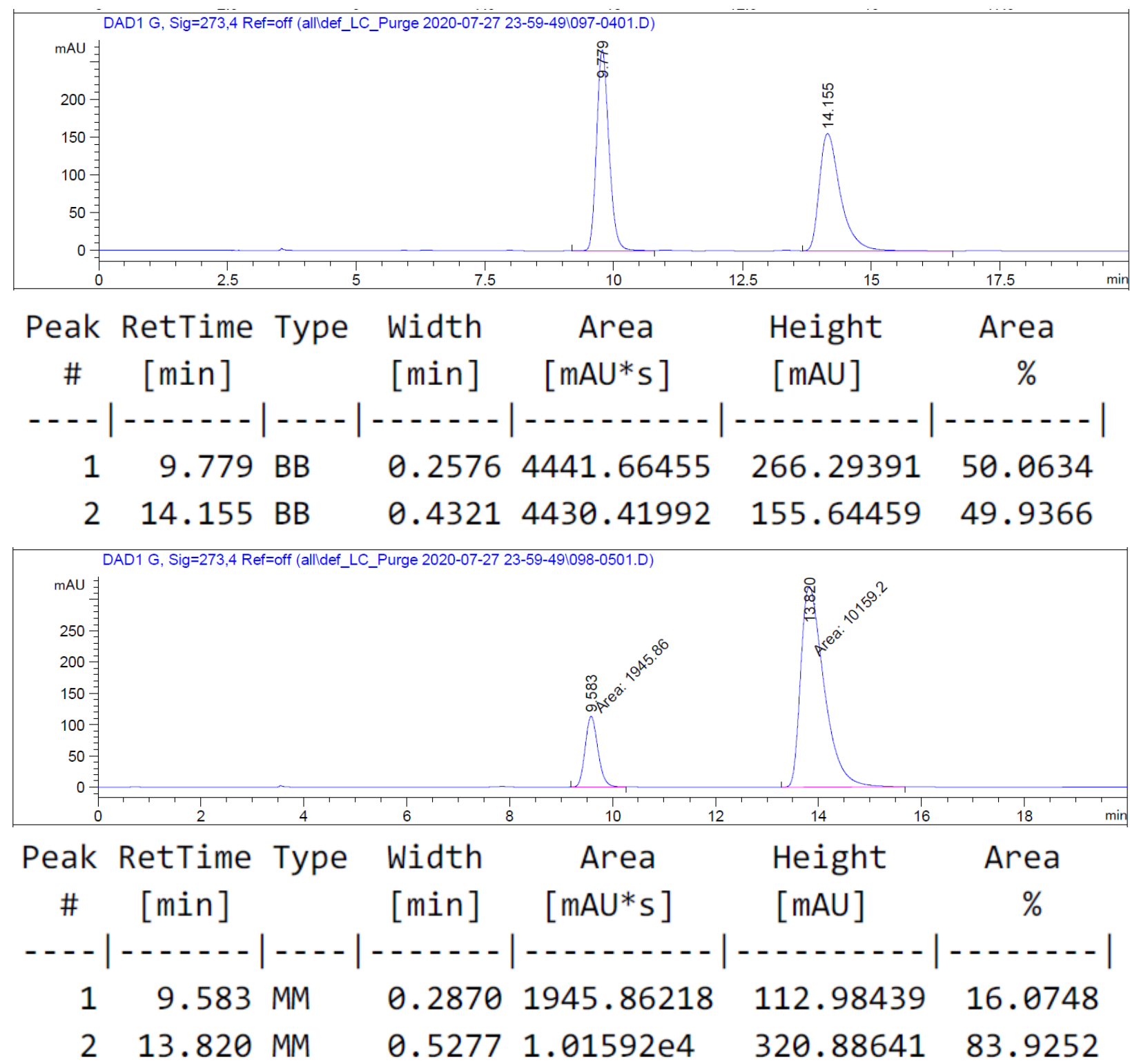




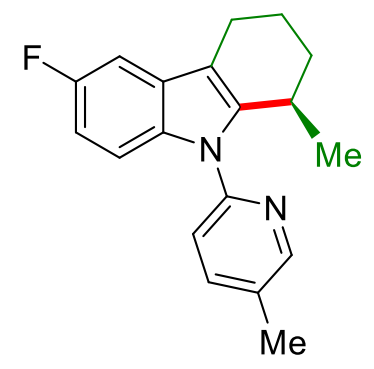

(R)-6-Fluoro-1-methyl-9-(5-methylpyridin-2-yl)-2,3,4,9-tetrahydro-1H-carbazole (2f): The general procedure A was followed using 5-fluoro-1-(5-methylpyridin-2-yl)-3-(pent-4-en-1-yl)$1 \mathrm{H}$-indole $(\mathbf{1 f})(73.5 \mathrm{mg}, 0.25 \mathrm{mmol})$. Isolation by column chromatography $(n$-hexane $/ \mathrm{EtOAc}=$ 30:1) yielded $2 \mathbf{f}(59.6 \mathrm{mg}, 81 \%)$ as a yellow oil. ${ }^{1} \mathrm{H}-\mathrm{NMR}\left(300 \mathrm{MHz}, \mathrm{CDCl}_{3}\right): \delta=8.47(\mathrm{~d}, J=2.4$ $\mathrm{Hz}, 1 \mathrm{H}), 7.70(\mathrm{dd}, J=8.1,2.4 \mathrm{~Hz}, 1 \mathrm{H}), 7.33(\mathrm{~d}, J=8.1 \mathrm{~Hz}, 1 \mathrm{H}), 7.27(\mathrm{dd}, J=8.7,4.2 \mathrm{~Hz}, 1 \mathrm{H})$, $7.16(\mathrm{dd}, J=9.4,2.6 \mathrm{~Hz}, 1 \mathrm{H}), 6.87(\mathrm{dd}, J=9.1,2.6 \mathrm{~Hz}, 1 \mathrm{H}), 3.52-3.41(\mathrm{~m}, 1 \mathrm{H}), 2.73$ (q, $J=6.1$, $5.7 \mathrm{~Hz}, 2 \mathrm{H}), 2.45(\mathrm{~s}, 3 \mathrm{H}), 2.17-2.04(\mathrm{~m}, 1 \mathrm{H}), 2.00-1.84(\mathrm{~m}, 2 \mathrm{H}), 1.73-1.61(\mathrm{~m}, 1 \mathrm{H}), 0.87(\mathrm{~d}, J=$ $6.9 \mathrm{~Hz}, 3 \mathrm{H}) .{ }^{13} \mathrm{C}-\mathrm{NMR}\left(100 \mathrm{MHz}, \mathrm{CDCl}_{3}\right): \delta=158.4\left(\mathrm{~d},{ }^{1} J_{\mathrm{C}-\mathrm{F}}=234.8 \mathrm{~Hz}, \mathrm{C}_{\mathrm{q}}\right), 149.9(\mathrm{CH}), 149.6$ $\left(\mathrm{C}_{\mathrm{q}}\right), 142.1\left(\mathrm{C}_{\mathrm{q}}\right), 139.1(\mathrm{CH}), 133.7\left(\mathrm{C}_{\mathrm{q}}\right), 131.6\left(\mathrm{C}_{\mathrm{q}}\right), 128.7\left(\mathrm{~d},{ }^{3} J_{\mathrm{C}-\mathrm{F}}=9.6 \mathrm{~Hz}, \mathrm{C}_{\mathrm{q}}\right), 119.9(\mathrm{CH})$, $112.2\left(\mathrm{~d},{ }^{4} J_{\mathrm{C}-\mathrm{F}}=4.3 \mathrm{~Hz}, \mathrm{C}_{\mathrm{q}}\right), 110.6\left(\mathrm{~d},{ }^{3} J_{\mathrm{C}-\mathrm{F}}=9.6 \mathrm{~Hz}, \mathrm{CH}\right), 109.5\left(\mathrm{~d},{ }^{2} J_{\mathrm{C}-\mathrm{F}}=25.8 \mathrm{~Hz}, \mathrm{CH}\right), 103.3$ $\left(\mathrm{d},{ }^{2} J_{\mathrm{C}-\mathrm{F}}=23.3 \mathrm{~Hz}, \mathrm{CH}\right), 31.8(\mathrm{CH}) 27.4\left(\mathrm{CH}_{2}\right), 21.5\left(\mathrm{CH}_{3}\right), 20.4\left(\mathrm{CH}_{2}\right), 20.0(\mathrm{CH}), 18.2\left(\mathrm{CH}_{3}\right)$. ${ }^{19} \mathrm{~F}-\mathrm{NMR}\left(282 \mathrm{MHz}, \mathrm{CDCl}_{3}\right.$ ): $\delta=-124.5$. IR (ATR): 1597, 1573, 1485, 1450, 1408, 1385, 1173, 798, $747 \mathrm{~cm}^{-1}$. MS (ESI-TOF) $\mathrm{m} / z$ (relative intensity): 295 (100) $[\mathrm{M}+\mathrm{H}]^{+}$. HR-MS (ESI-TOF): $m / z:[\mathrm{M}+\mathrm{H}]^{+}$Calcd. for $\mathrm{C}_{19} \mathrm{H}_{20}{ }^{19} \mathrm{FN}_{2}$ 295.1611; Found: 295.1605. $[\alpha]_{\mathrm{D}}^{20}:-29.9\left(\mathrm{c}=0.8, \mathrm{CHCl}_{3}\right)$. HPLC separation $\left(\right.$ Chiralpak $^{\circledR}$ ID-3, $n$-hexane/EtOAc 90:10, $1.0 \mathrm{~mL} / \mathrm{min}$, detection at $273 \mathrm{~nm}$ ): $t_{r}$ $($ major $)=8.0 \mathrm{~min}, t_{r}($ minor $)=5.8 \mathrm{~min}, 82: 18$. e.r.

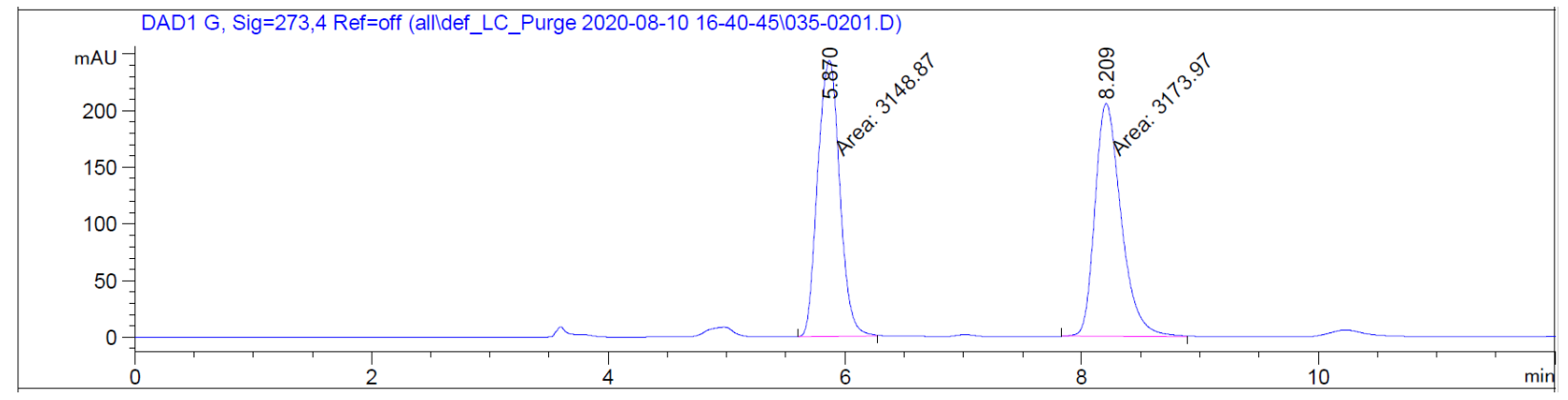



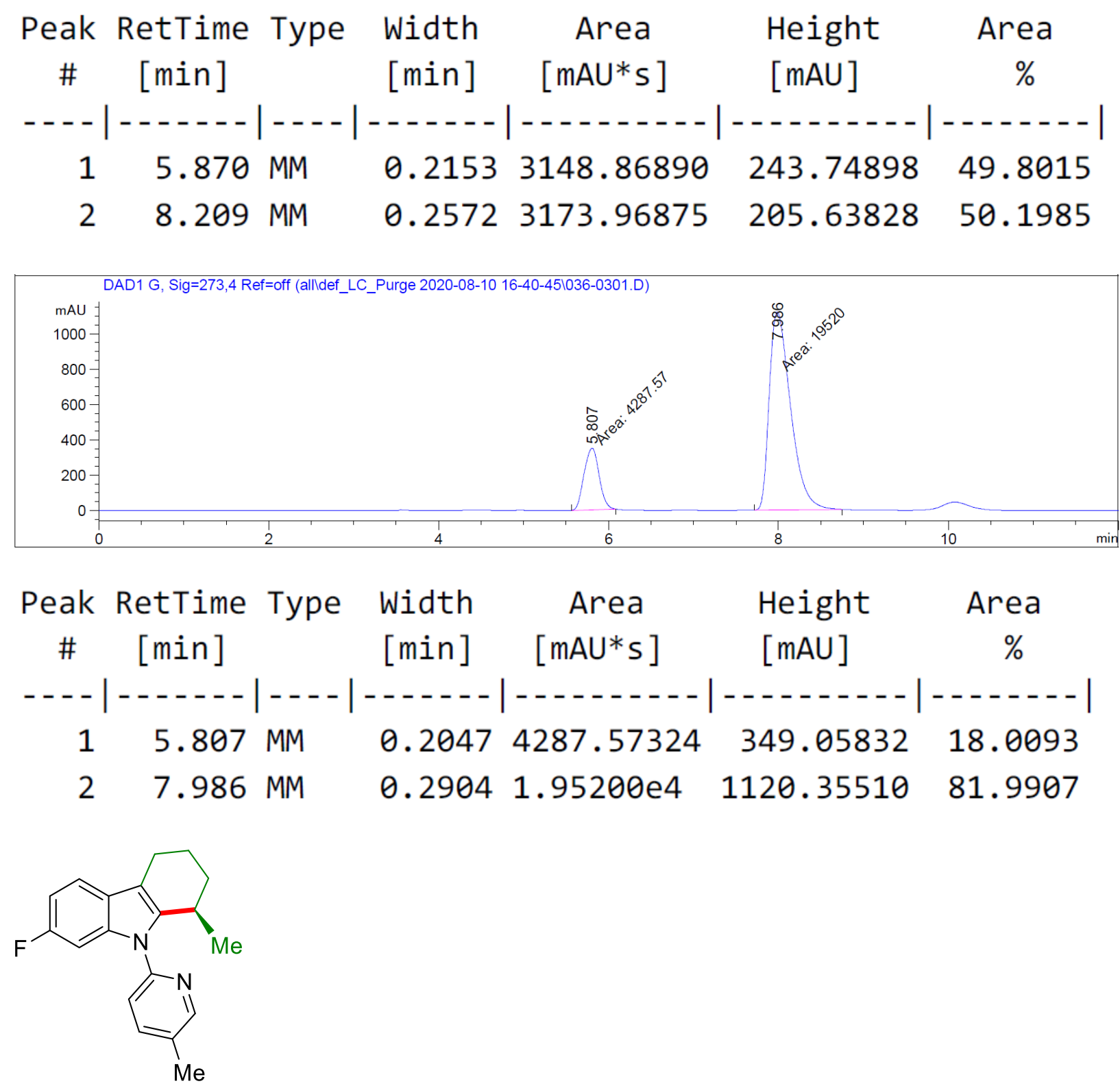

(R)-7-Fluoro-1-methyl-9-(5-methylpyridin-2-yl)-2,3,4,9-tetrahydro-1H-carbazole (2g): The general procedure A was followed using 6-fluoro-1-(5-methylpyridin-2-yl)-3-(pent-4-en-1-yl)$1 H$-indole $(\mathbf{1 g})(73.5 \mathrm{mg}, 0.25 \mathrm{mmol})$. Isolation by column chromatography $(n$-hexane/EtOAc $=$ 30:1) yielded $2 \mathrm{~g}(49 \mathrm{mg}, 66 \%)$ as a yellow oil. ${ }^{1} \mathrm{H}-\mathrm{NMR}\left(400 \mathrm{MHz}, \mathrm{CDCl}_{3}\right): \delta=8.47(\mathrm{~d}, J=2.3$ $\mathrm{Hz}, 1 \mathrm{H}), 7.71(\mathrm{dd}, J=8.1,2.3 \mathrm{~Hz}, 1 \mathrm{H}), 7.41(\mathrm{dd}, J=8.5,5.4 \mathrm{~Hz}, 1 \mathrm{H}), 7.33$ (d, $J=8.0 \mathrm{~Hz}, 1 \mathrm{H})$, 7.07 (dd, $J=9.5,2.3 \mathrm{~Hz}, 1 \mathrm{H}), 6.90(\mathrm{ddd}, J=9.5,8.5,2.3 \mathrm{~Hz}, 1 \mathrm{H}), 3.55-3.38(\mathrm{~m}, 1 \mathrm{H}), 2.84-2.66$ $(\mathrm{m}, 2 \mathrm{H}), 2.45(\mathrm{~s}, 3 \mathrm{H}), 2.16-2.05(\mathrm{~m}, 1 \mathrm{H}), 2.00-1.81(\mathrm{~m}, 2 \mathrm{H}), 1.69-1.59(\mathrm{~m}, 1 \mathrm{H}), 0.85(\mathrm{~d}, J=6.8$ $\mathrm{Hz}, 3 \mathrm{H}) .{ }^{13} \mathrm{C}-\mathrm{NMR}\left(75 \mathrm{MHz}, \mathrm{CDCl}_{3}\right): \delta=160.1\left(\mathrm{~d},{ }^{1} J_{\mathrm{C}-\mathrm{F}}=234.1 \mathrm{~Hz}, \mathrm{C}_{\mathrm{q}}\right), 149.9(\mathrm{CH}), 149.4\left(\mathrm{C}_{\mathrm{q}}\right)$, $140.5\left(\mathrm{~d},{ }^{4} J_{\mathrm{C}-\mathrm{F}}=3.4 \mathrm{~Hz}, \mathrm{C}_{\mathrm{q}}\right), 139.0(\mathrm{CH}), 137.1\left(\mathrm{~d},{ }^{3} J_{\mathrm{C}-\mathrm{F}}=12.2 \mathrm{~Hz}, \mathrm{C}_{\mathrm{q}}\right), 131.5\left(\mathrm{C}_{\mathrm{q}}\right), 124.6\left(\mathrm{C}_{\mathrm{q}}\right)$, 
$119.6(\mathrm{CH}), 118.5\left(\mathrm{~d},{ }^{3} J_{\mathrm{C}-\mathrm{F}}=9.9 \mathrm{~Hz}, \mathrm{CH}\right), 112.1\left(\mathrm{C}_{\mathrm{q}}\right), 108.1\left(\mathrm{~d},{ }^{2} J_{\mathrm{C}-\mathrm{F}}=24.1 \mathrm{~Hz}, \mathrm{CH}\right), 97.0(\mathrm{~d}$, $\left.{ }^{2} J_{\mathrm{C}-\mathrm{F}}=27.2 \mathrm{~Hz}, \mathrm{CH}\right), 31.7\left(\mathrm{CH}_{2}\right), 27.2(\mathrm{CH}), 21.4\left(\mathrm{CH}_{2}\right), 20.3\left(\mathrm{CH}_{3}\right), 19.9\left(\mathrm{CH}_{2}\right), 18.1\left(\mathrm{CH}_{3}\right) .{ }^{19} \mathrm{~F}-$ NMR (377 MHz, CDCl 3 ): $\delta=-121.5$. IR (ATR): 2929, 1596, 1473, 1484, 1387, 1142, 825, 797 $\mathrm{cm}^{-1}$. MS (ESI-TOF) $\mathrm{m} / \mathrm{z}$ (relative intensity): 295 (100) $[\mathrm{M}+\mathrm{H}]^{+}$. HR-MS (ESI-TOF) $\mathrm{m} / \mathrm{z}:[\mathrm{M}+$ $\mathrm{H}]^{+}$Calcd. for $\mathrm{C}_{19} \mathrm{H}_{20}{ }^{19} \mathrm{FN}_{2}$ 295.1605; Found 295.1612. $[\alpha]_{\mathrm{D}}^{20}:-78.6\left(\mathrm{c}=1.0, \mathrm{CHCl}_{3}\right)$. HPLC separation (Chiralpak ${ }^{\circledR} \mathrm{ID}-3, n$-hexane/EtOAc 90:10, $1.0 \mathrm{~mL} / \mathrm{min}$, detection at $273 \mathrm{~nm}$ ): $t_{r}$ (major) $=7.2 \mathrm{~min}, t_{r}($ minor $)=5.3 \mathrm{~min}, 86: 14$ e.r.
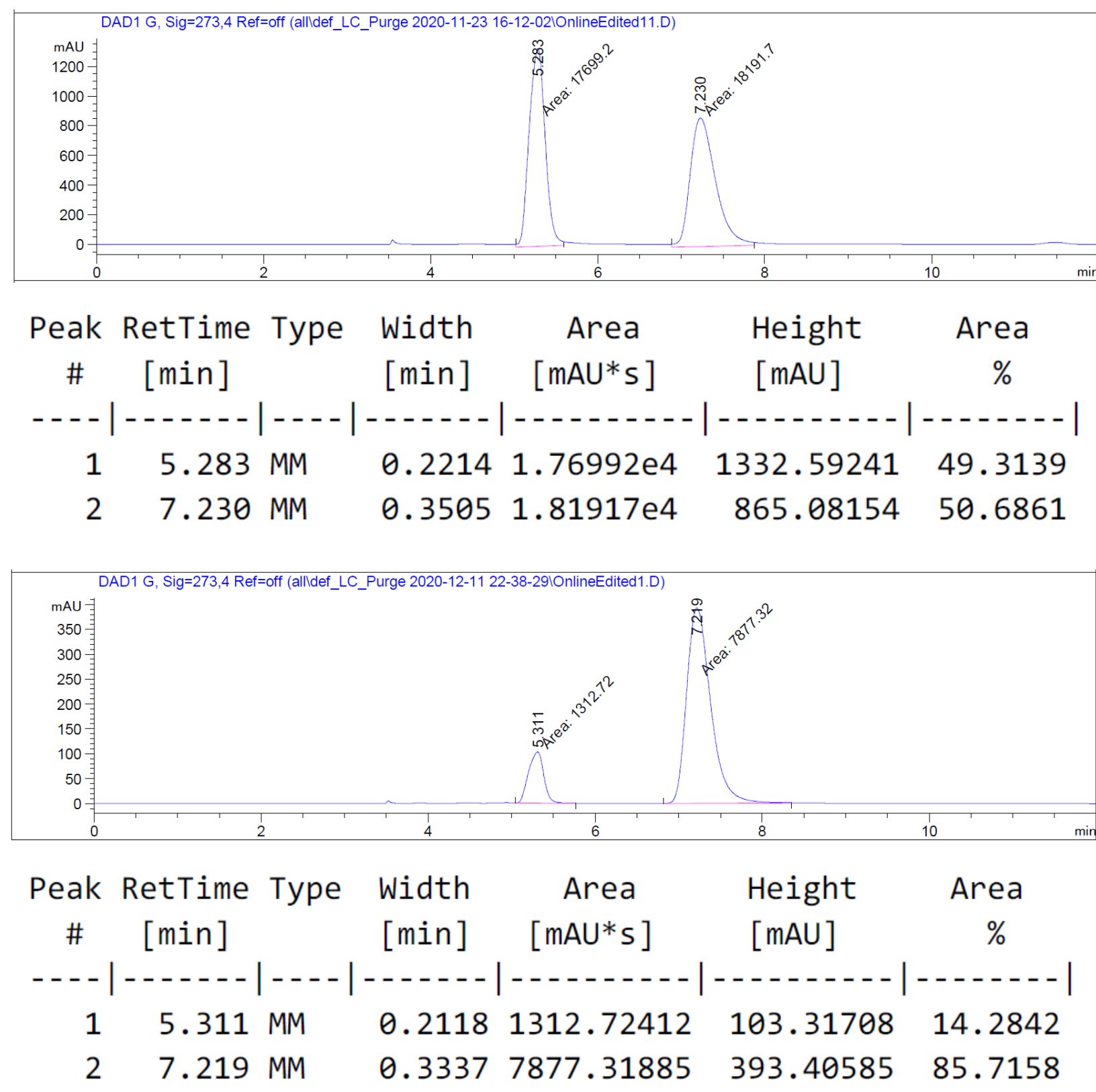


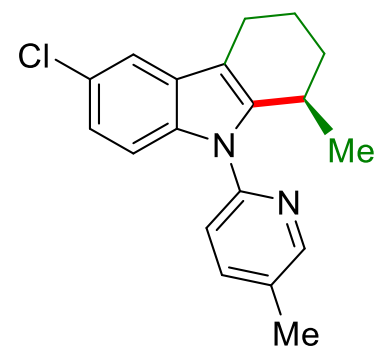

(R)-6-Chloro-1-methyl-9-(5-methylpyridin-2-yl)-2,3,4,9-tetrahydro-1H-carbazole (2h): The general procedure $\mathbf{A}$ was followed using 5-chloro-1-(5-methylpyridin-2-yl)-3-(pent-4-en-1-yl)$1 H$-indole $(\mathbf{1 h})(77.8 \mathrm{mg}, 0.25 \mathrm{mmol})$. Isolation by column chromatography $(n$-hexane/EtOAc $=$ 20:1) yielded $2 \mathbf{h}(71.0 \mathrm{mg}, 91 \%)$ as a white solid. M.p.: $123-126{ }^{\circ} \mathrm{C} .{ }^{1} \mathrm{H}-\mathrm{NMR}\left(400 \mathrm{MHz}, \mathrm{CDCl}_{3}\right)$ : $\delta=8.45(\mathrm{dd}, J=2.4,0.8 \mathrm{~Hz}, 1 \mathrm{H}), 7.68(\mathrm{ddd}, J=8.1,2.4,0.8 \mathrm{~Hz}, 1 \mathrm{H}), 7.45(\mathrm{~d}, J=2.1 \mathrm{~Hz}, 1 \mathrm{H})$, $7.30(\mathrm{~d}, J=8.1 \mathrm{~Hz}, 1 \mathrm{H}), 7.22(\mathrm{~d}, J=8.6 \mathrm{~Hz}, 1 \mathrm{H}), 7.05(\mathrm{dd}, J=8.6,2.1 \mathrm{~Hz}, 1 \mathrm{H}), 3.56-3.35(\mathrm{~m}$, $1 \mathrm{H}), 2.78-2.62(\mathrm{~m}, 2 \mathrm{H}), 2.43(\mathrm{~s}, 3 \mathrm{H}), 2.14-2.02(\mathrm{~m}, 1 \mathrm{H}), 1.97-1.75(\mathrm{~m}, 2 \mathrm{H}), 1.69-1.58(\mathrm{~m}, 1 \mathrm{H})$, $0.83(\mathrm{~d}, J=6.9 \mathrm{~Hz}, 3 \mathrm{H}) .{ }^{13} \mathrm{C}-\mathrm{NMR}\left(100 \mathrm{MHz}, \mathrm{CDCl}_{3}\right): \delta=149.7(\mathrm{CH}), 149.2\left(\mathrm{C}_{\mathrm{q}}\right), 141.7\left(\mathrm{C}_{\mathrm{q}}\right)$, $139.1(\mathrm{CH}), 135.4\left(\mathrm{C}_{\mathrm{q}}\right), 131.6\left(\mathrm{C}_{\mathrm{q}}\right), 129.2\left(\mathrm{C}_{\mathrm{q}}\right), 125.6\left(\mathrm{C}_{\mathrm{q}}\right), 121.7(\mathrm{CH}), 119.8(\mathrm{CH}), 117.6(\mathrm{CH})$, $111.8\left(\mathrm{C}_{\mathrm{q}}\right), 110.8(\mathrm{CH}), 31.6\left(\mathrm{CH}_{2}\right), 27.1(\mathrm{CH}), 21.3\left(\mathrm{CH}_{2}\right), 20.2\left(\mathrm{CH}_{3}\right), 19.7\left(\mathrm{CH}_{2}\right), 18.0\left(\mathrm{CH}_{3}\right)$. IR (ATR): 2929, 2856, 1596, 1483, 1403, 1386, 1223, 1067, $795 \mathrm{~cm}^{-1}$. MS (ESI-TOF) $\mathrm{m} / \mathrm{z}$ (relative intensity): $643(15)[2 \mathrm{M}+\mathrm{Na}]^{+}, 621(10)[2 \mathrm{M}+\mathrm{H}]^{+}, 333(20)[\mathrm{M}+\mathrm{Na}]^{+}, 311(100)[\mathrm{M}+\mathrm{H}]^{+}$. HR-MS (ESI-TOF): $m / z[\mathrm{M}+\mathrm{H}]^{+}$Calcd. for $\mathrm{C}_{19} \mathrm{H}_{20}{ }^{35} \mathrm{ClN}_{2}$ 311.1310; Found 311.1311. [ $\left.\alpha\right]_{\mathrm{D}}^{20}$ :$78.2\left(\mathrm{c}=1.0, \mathrm{CHCl}_{3}\right)$. HPLC separation (Chiralpak ${ }^{\circledR}$ ID-3, $n$-hexane/EtOAc 90:10, $1.0 \mathrm{~mL} / \mathrm{min}$, detection at $273 \mathrm{~nm}): t_{r}$ (major) $=8.8 \mathrm{~min}, t_{r}($ minor $)=5.9 \mathrm{~min}, 82: 18$ e.r.

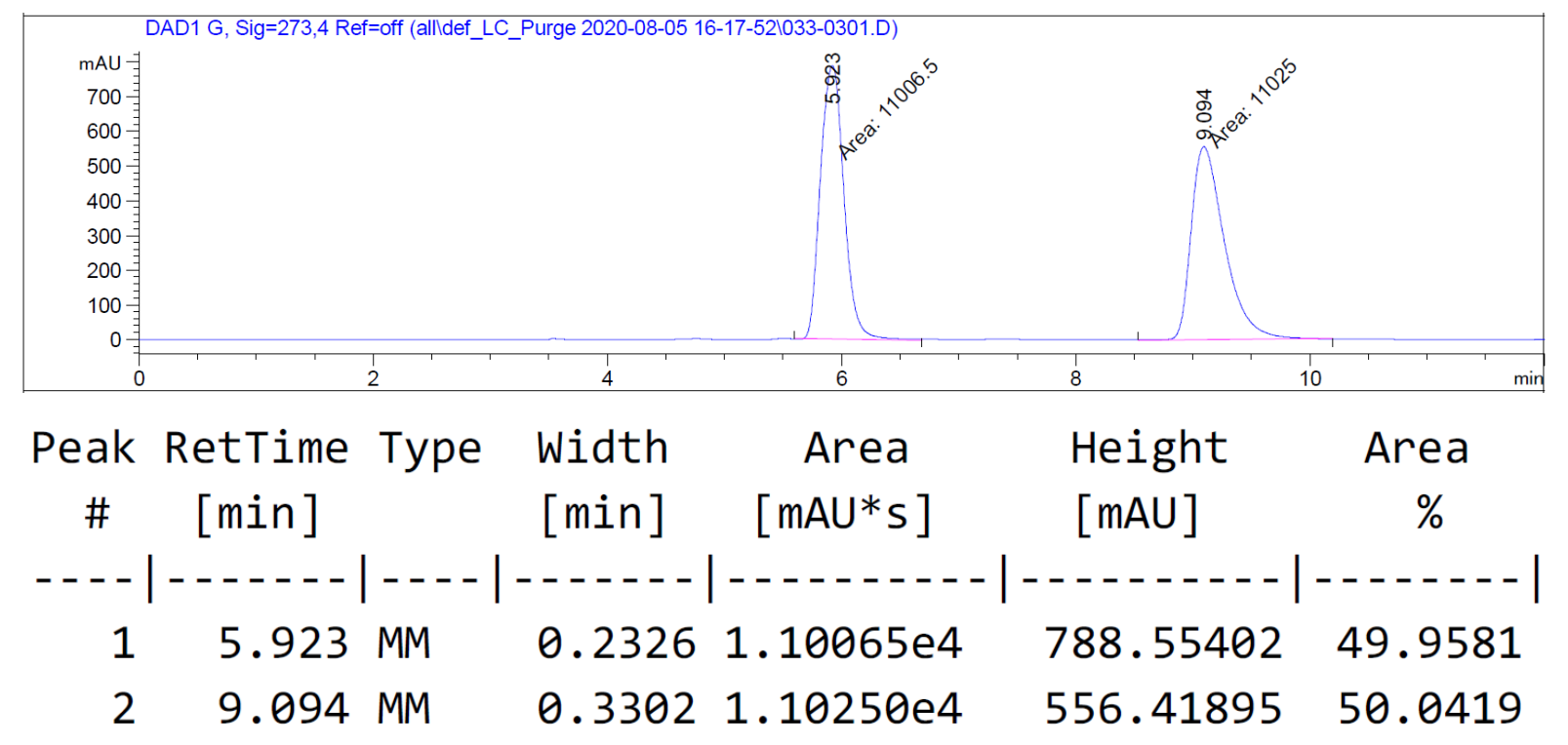




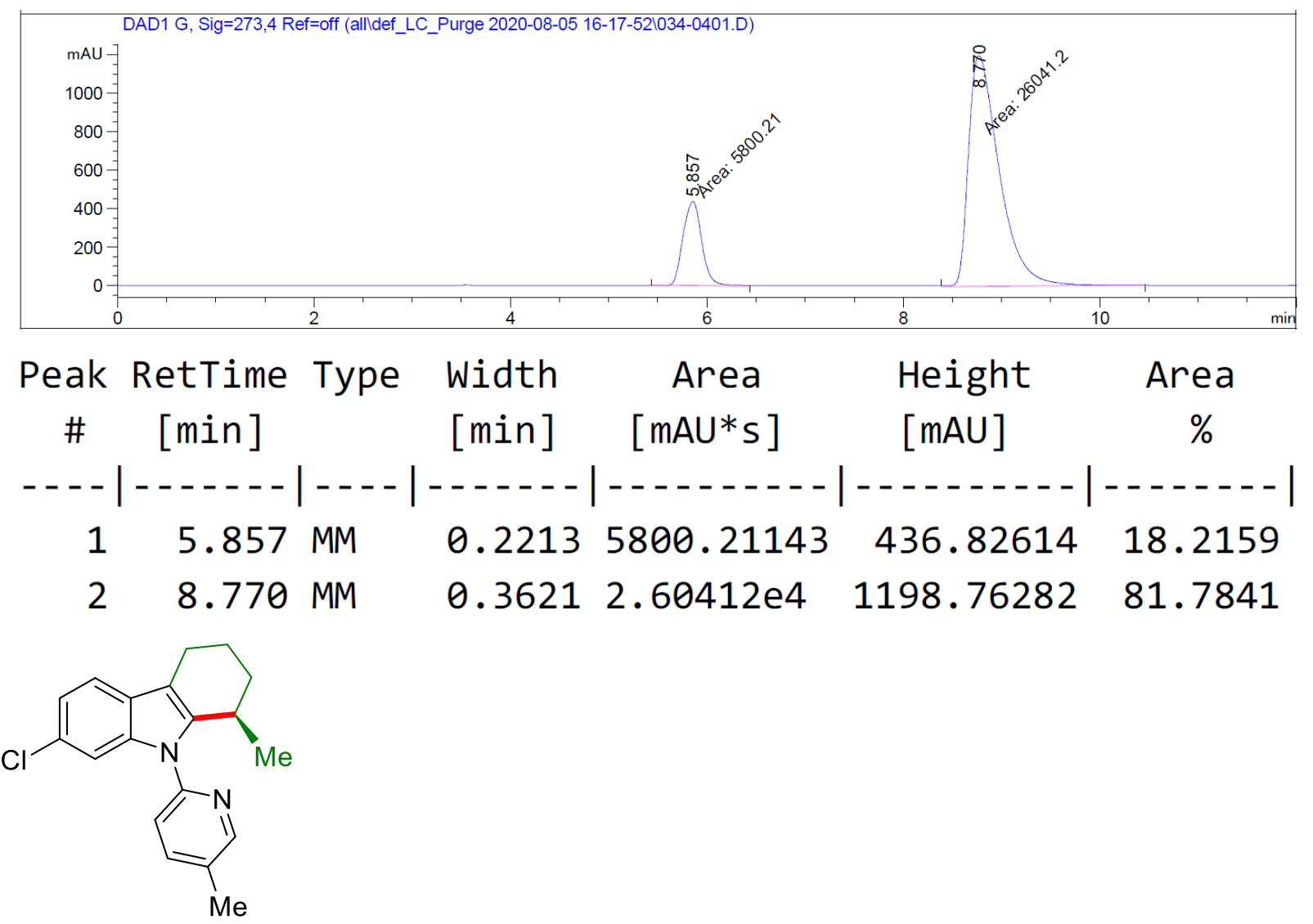

(R)-7-Chloro-1-methyl-9-(5-methylpyridin-2-yl)-2,3,4,9-tetrahydro-1H-carbazole (2i): The general procedure A was followed using 6-chloro-1-(5-methylpyridin-2-yl)-3-(pent-4-en-1-yl)$1 H$-indole $(\mathbf{1 i})(77.8 \mathrm{mg}, 0.25 \mathrm{mmol})$. Isolation by column chromatography $(n$-hexane/EtOAc $=$ 20:1) yielded $2 \mathbf{i}(70.3 \mathrm{mg}, 90 \%)$ as a white solid. M.p.: 96-98 ${ }^{\circ} \mathrm{C} .{ }^{1} \mathrm{H}-\mathrm{NMR}\left(400 \mathrm{MHz}, \mathrm{CDCl}_{3}\right): \delta$ $=8.45(\mathrm{~d}, J=2.4 \mathrm{~Hz}, 1 \mathrm{H}), 7.69(\mathrm{dd}, J=8.1,2.4 \mathrm{~Hz}, 1 \mathrm{H}), 7.39(\mathrm{~d}, J=8.3 \mathrm{~Hz}, 1 \mathrm{H}), 7.34-7.27(\mathrm{~m}$, 2H), $7.08(\mathrm{dd}, J=8.3,1.8 \mathrm{~Hz}, 1 \mathrm{H}), 3.49-3.30(\mathrm{~m}, 1 \mathrm{H}), 2.88-2.61(\mathrm{~m}, 2 \mathrm{H}), 2.43(\mathrm{~s}, 3 \mathrm{H}), 2.17-2.01$ (m, 1H), 1.98-1.79 (m, 2H), 1.72-1.57 (m, 1H), $0.83(\mathrm{~d}, J=6.9 \mathrm{~Hz}, 3 \mathrm{H}) .{ }^{13} \mathrm{C}-\mathrm{NMR}(100 \mathrm{MHz}$, $\left.\mathrm{CDCl}_{3}\right): \delta=149.9(\mathrm{CH}), 149.1\left(\mathrm{C}_{\mathrm{q}}\right), 140.9\left(\mathrm{C}_{\mathrm{q}}\right), 139.0(\mathrm{CH}), 137.4\left(\mathrm{C}_{\mathrm{q}}\right), 131.7\left(\mathrm{C}_{\mathrm{q}}\right), 127.5\left(\mathrm{C}_{\mathrm{q}}\right)$, $126.6\left(\mathrm{C}_{\mathrm{q}}\right), 120.4(\mathrm{CH}), 119.8(\mathrm{CH}), 118.8(\mathrm{CH}), 112.1\left(\mathrm{C}_{\mathrm{q}}\right), 110.0(\mathrm{CH}), 31.6\left(\mathrm{CH}_{2}\right), 27.1(\mathrm{CH})$, $21.3\left(\mathrm{CH}_{2}\right), 20.2\left(\mathrm{CH}_{3}\right), 19.8\left(\mathrm{CH}_{2}\right), 18.0\left(\mathrm{CH}_{3}\right)$. IR (ATR): 2929, 2858, 1596, 1483, 1386, 1222 , $1029 \mathrm{~cm}^{-1}$. MS (ESI-TOF) $\mathrm{m} / z$ (relative intensity): $643(5)[2 \mathrm{M}+\mathrm{Na}]^{+}, 621(10)[2 \mathrm{M}+\mathrm{H}]^{+}, 333$ (15) $[\mathrm{M}+\mathrm{Na}]^{+}, 311(100)[\mathrm{M}+\mathrm{H}]^{+}$. HR-MS (ESI-TOF) $m / z:[\mathrm{M}+\mathrm{Na}]^{+}$Calcd. for $\mathrm{C}_{19} \mathrm{H}_{19}{ }^{35} \mathrm{ClN}_{2} \mathrm{Na}$ 333.1129; Found 333.1125. $[\alpha]_{\mathrm{D}}^{20}:-83.7\left(\mathrm{c}=1.0, \mathrm{CHCl}_{3}\right)$. HPLC separation $\left(\right.$ Chiralpak $^{\circledR} \mathrm{ID}-3, n$-hexane/EtOAc 90:10, $1.0 \mathrm{~mL} / \mathrm{min}$, detection at $\left.273 \mathrm{~nm}\right): t_{r}$ (major) $=7.2 \mathrm{~min}$, $t_{r}($ minor $)=5.3 \mathrm{~min}, 83: 17$ e.r. 

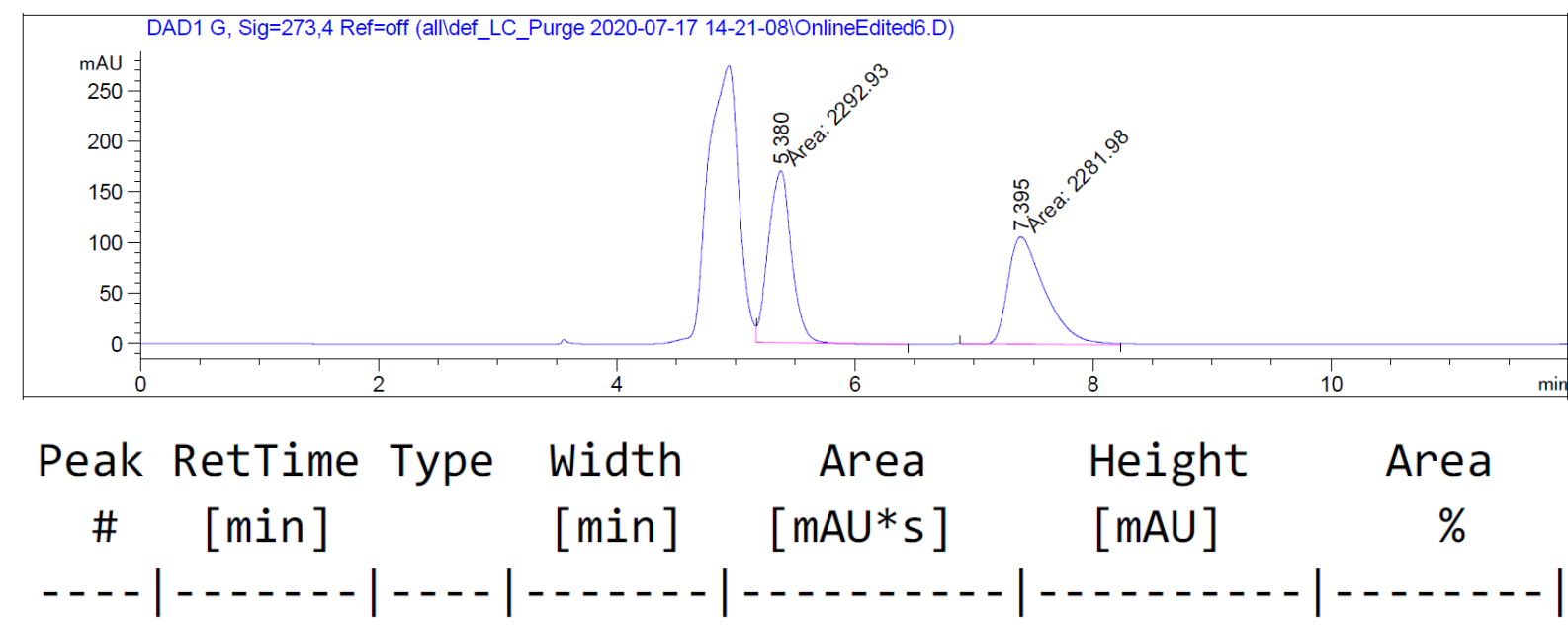

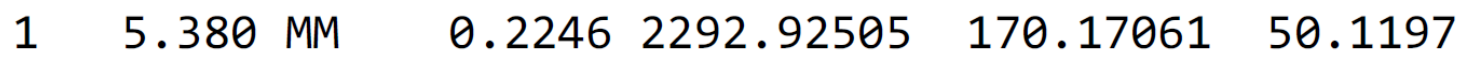

$\begin{array}{lllllll}2 & 7.395 & \text { MM } & 0.3586 & 2281.97583 & 106.04570 & 49.8803\end{array}$

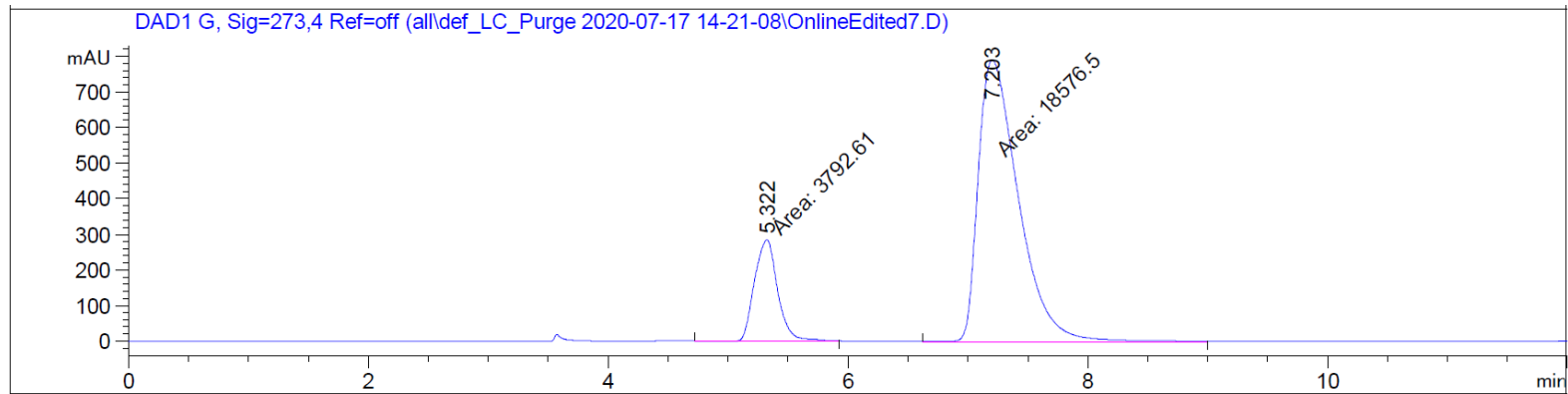

Peak RetTime Type Width Area Height Area

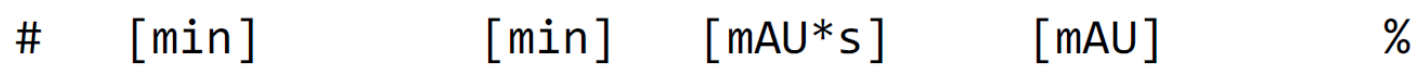

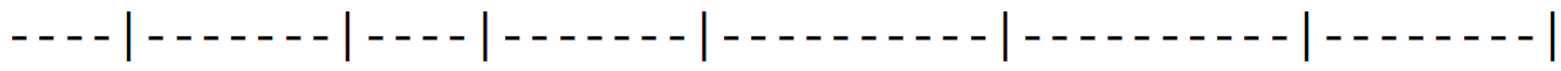

$\begin{array}{lllllll}1 & 5.322 & \text { MM } & 0.2226 & 3792.60889 & 283.95197 & 16.9547\end{array}$

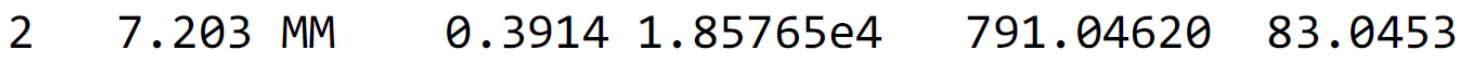<smiles>[M]C1CCCc2c1n(-c1ccc([N+](=O)[O-])cn1)c1ccc(OCC(C)C)cc21</smiles>

\section{(R)-6-[(tert-Butyldimethylsilyl)oxy]-1-methyl-9-(5-methylpyridin-2-yl)-2,3,4,9-tetrahydro-}

$\mathbf{1 H}$-carbazole (2j): The general procedure A was followed using 5-[(tert-butyldimethylsilyl)oxy]1-(5-methylpyridin-2-yl)-3-(pent-4-en-1-yl)-1H-indole (1j) (101.7 mg, $0.25 \mathrm{mmol})$. Isolation by column chromatography ( $n$-hexane/EtOAc $=20: 1)$ yielded $\mathbf{2 j}(100 \mathrm{mg}, 99 \%)$ as a white solid. M.p.: 101-104 ${ }^{\circ} \mathrm{C} .{ }^{1} \mathrm{H}-\mathrm{NMR}\left(400 \mathrm{MHz}, \mathrm{CDCl}_{3}\right): \delta=8.40(\mathrm{dd}, J=2.4,0.7 \mathrm{~Hz}, 1 \mathrm{H}), 7.63(\mathrm{ddd}, J=8.1$, 
2.4, $0.7 \mathrm{~Hz}, 1 \mathrm{H}), 7.30(\mathrm{~d}, J=8.1 \mathrm{~Hz}, 1 \mathrm{H}), 7.17(\mathrm{dd}, J=8.7,0.6 \mathrm{~Hz}, 1 \mathrm{H}), 6.89(\mathrm{dd}, J=2.4,0.6 \mathrm{~Hz}$, 1H), $6.64(\mathrm{dd}, J=8.7,2.4 \mathrm{~Hz}, 1 \mathrm{H}), 3.44(\mathrm{~h}, J=6.4 \mathrm{~Hz}, 1 \mathrm{H}), 2.74-2.59(\mathrm{~m}, 2 \mathrm{H}), 2.39$ (s, 3H), 2.13-2.01 (m, 1H), 2.00-1.77 (m, 2H), 1.65-1.55 (m, 1H), 0.99 (s, 9H), 0.82 (d, $J=6.8 \mathrm{~Hz}, 3 \mathrm{H})$, $0.17(\mathrm{~s}, 6 \mathrm{H}) .{ }^{13} \mathrm{C}-\mathrm{NMR}\left(100 \mathrm{MHz}, \mathrm{CDCl}_{3}\right): \delta=150.0\left(\mathrm{C}_{\mathrm{q}}\right), 149.8\left(\mathrm{C}_{\mathrm{q}}\right), 149.7(\mathrm{CH}), 140.9\left(\mathrm{C}_{\mathrm{q}}\right)$, 139.0 (CH), $132.7\left(\mathrm{C}_{\mathrm{q}}\right), 131.0\left(\mathrm{C}_{\mathrm{q}}\right), 129.0\left(\mathrm{C}_{\mathrm{q}}\right), 119.7(\mathrm{CH}), 115.5(\mathrm{CH}), 112.1\left(\mathrm{C}_{\mathrm{q}}\right), 110.3(\mathrm{CH})$, $108.2(\mathrm{CH}), 32.0\left(\mathrm{CH}_{2}\right), 27.4(\mathrm{CH}), 26.0\left(\mathrm{CH}_{3}\right), 21.7\left(\mathrm{CH}_{2}\right), 20.5\left(\mathrm{CH}_{3}\right), 20.1\left(\mathrm{CH}_{2}\right), 18.4\left(\mathrm{C}_{\mathrm{q}}\right)$, $18.2\left(\mathrm{CH}_{3}\right),-4.2\left(\mathrm{CH}_{3}\right)$. IR (ATR): 2928, 1597, 1484, 1463, 1255, 1195, $835 \mathrm{~cm}^{-1}$. MS (ESI-TOF) $\mathrm{m} / \mathrm{z}$ (relative intensity): $836(20)[2 \mathrm{M}+\mathrm{Na}]^{+}, 814(80)[2 \mathrm{M}+\mathrm{H}]^{+}, 429(15)[\mathrm{M}+\mathrm{Na}]^{+}, 407(100)$ $[\mathrm{M}+\mathrm{H}]^{+}$. HR-MS (ESI-TOF) $m / z:[\mathrm{M}+\mathrm{H}]^{+}$Calcd. for $\mathrm{C}_{25} \mathrm{H}_{35} \mathrm{~N}_{2} \mathrm{O}^{29} \mathrm{Si}$ 407.2513; Found 407.2515. $[\alpha]_{\mathrm{D}}^{20}:-55.4\left(\mathrm{c}=1.0, \mathrm{CHCl}_{3}\right)$. HPLC separation $\left(\right.$ Chiralpak $^{\circledR}$ IB-3, $n$-hexane/EtOAc 95:5, 1.0 $\mathrm{mL} / \mathrm{min}$, detection at $273 \mathrm{~nm}$ ): $t_{r}($ minor $)=8.7 \mathrm{~min}, t_{r}($ major $)=11.3 \mathrm{~min}, 85: 15$ e.r.

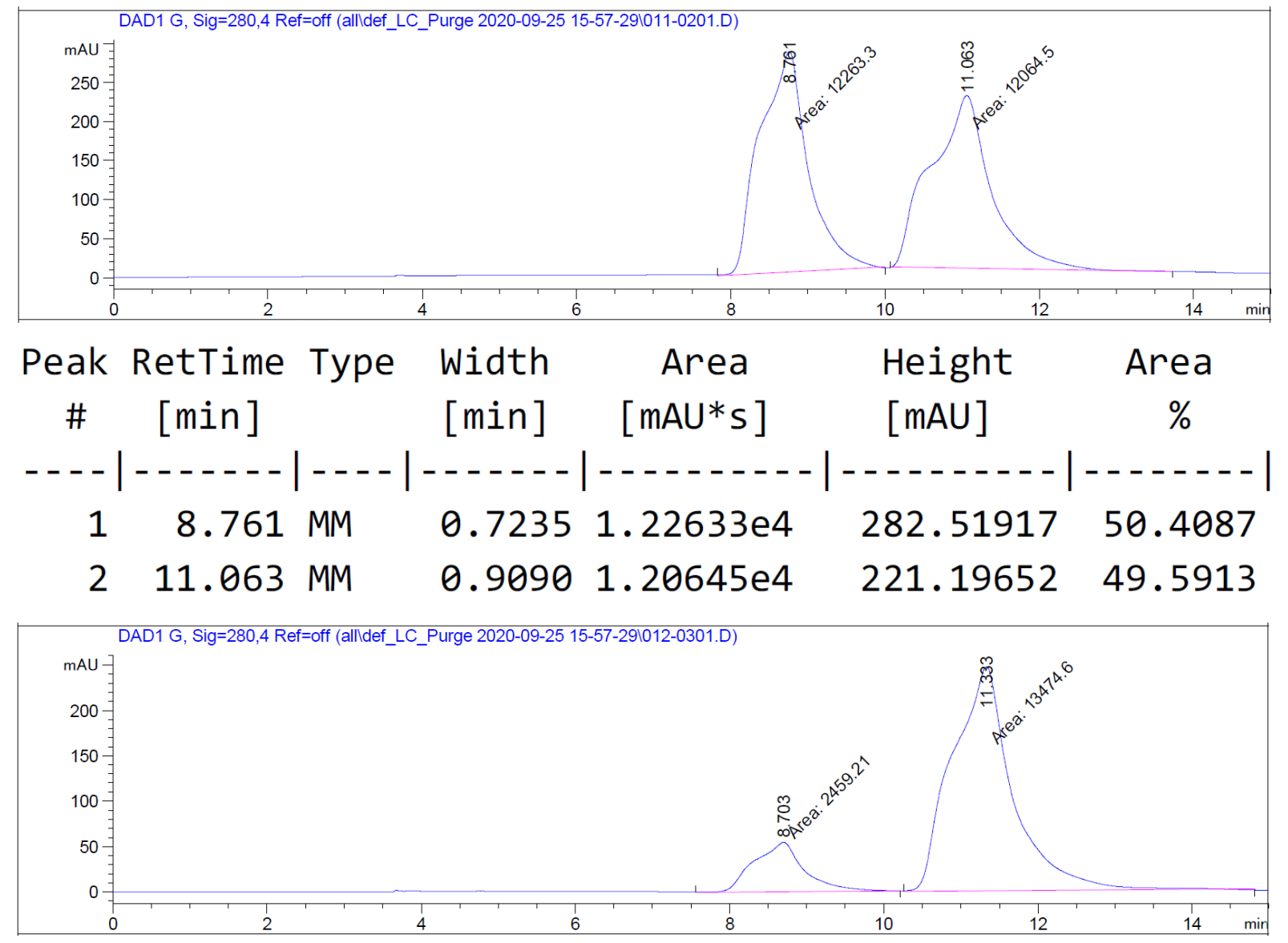




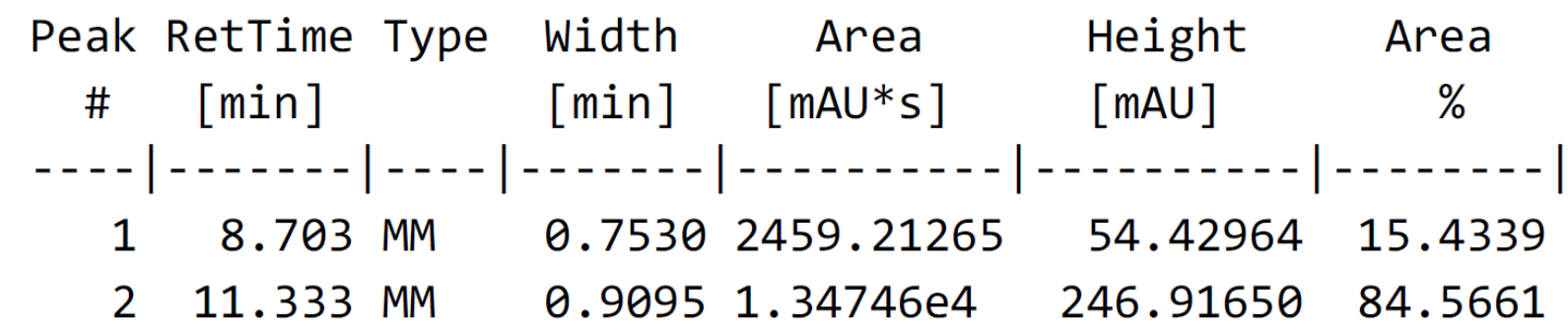<smiles>Cc1ccc(-n2c3c(c4cc(Br)ccc42)CCC[C@@H]3C)nc1</smiles>

(R)-6-Bromo-1-methyl-9-(5-methylpyridin-2-yl)-2,3,4,9-tetrahydro-1H-carbazole (2k): The general procedure A was followed using 5-bromo-1-(5-methylpyridin-2-yl)-3-(pent-4-en-1-yl)$1 H$-indole $(\mathbf{1 k})(88.5 \mathrm{mg}, 0.25 \mathrm{mmol})$. Isolation by column chromatography $(n$-hexane/EtOAc $=$ 15:1) yielded 2k (78.0 mg, 88\%) as a yellow solid. M.p.: $110-115{ }^{\circ} \mathrm{C}$. ${ }^{1} \mathrm{H}-\mathrm{NMR}(300 \mathrm{MHz}$, $\left.\mathrm{CDCl}_{3}\right): \delta=8.44(\mathrm{~d}, J=2.4 \mathrm{~Hz}, 1 \mathrm{H}), 7.68(\mathrm{dd}, J=8.2,2.4 \mathrm{~Hz}, 1 \mathrm{H}), 7.60(\mathrm{~d}, J=1.3 \mathrm{~Hz}, 1 \mathrm{H}), 7.29$ $(\mathrm{d}, J=8.2 \mathrm{~Hz}, 1 \mathrm{H}), 7.23-7.10(\mathrm{~m}, 2 \mathrm{H}), 3.52-3.34(\mathrm{~m}, 1 \mathrm{H}), 2.81-2.59$ (m, 2H), $2.43(\mathrm{~s}, 3 \mathrm{H}), 2.15-$ $2.01(\mathrm{~m}, 1 \mathrm{H}), 1.97-1.80(\mathrm{~m}, 2 \mathrm{H}), 1.74-1.55(\mathrm{~m}, 1 \mathrm{H}), 0.83(\mathrm{~d}, J=6.8 \mathrm{~Hz}, 3 \mathrm{H}) .{ }^{13} \mathrm{C}-\mathrm{NMR}(100$ $\left.\mathrm{MHz}, \mathrm{CDCl}_{3}\right): \delta=149.9(\mathrm{CH}), 149.3\left(\mathrm{C}_{\mathrm{q}}\right), 141.6\left(\mathrm{C}_{\mathrm{q}}\right), 139.0(\mathrm{CH}), 135.7\left(\mathrm{C}_{\mathrm{q}}\right), 131.7\left(\mathrm{C}_{\mathrm{q}}\right), 129.9$ $\left(\mathrm{C}_{\mathrm{q}}\right), 124.3(\mathrm{CH}), 120.7(\mathrm{CH}), 119.8(\mathrm{CH}), 113.2\left(\mathrm{C}_{\mathrm{q}}\right), 111.7\left(\mathrm{C}_{\mathrm{q}}\right), 111.3(\mathrm{CH}), 31.6\left(\mathrm{CH}_{2}\right), 27.1$ (CH), $21.3\left(\mathrm{CH}_{2}\right), 20.2\left(\mathrm{CH}_{3}\right), 19.8\left(\mathrm{CH}_{2}\right), 18.1\left(\mathrm{CH}_{3}\right)$. IR (ATR): 2929, 1572, 1483, 1455, 1402, 1027, $792 \mathrm{~cm}^{-1}$. MS (ESI-TOF) $\mathrm{m} / z$ (relative intensity): $377(25)[\mathrm{M}+\mathrm{Na}]^{+}, 355(100)[\mathrm{M}+\mathrm{H}]^{+}$. HR-MS (ESI-TOF) $m / z$ : $[\mathrm{M}+\mathrm{Na}]^{+}$Calcd. for $\mathrm{C}_{19} \mathrm{H}_{19}{ }^{79} \mathrm{BrN}_{2} \mathrm{Na} 377.0624$; Found 377.0620. $[\alpha]_{\mathrm{D}}^{20}$ : -50.5 (c = 1.0, $\left.\mathrm{CHCl}_{3}\right)$. HPLC separation (Chiralpak ${ }^{\circledR} \mathrm{ID}-3, n$-hexane/EtOAc 90:10, $1.0 \mathrm{~mL} / \mathrm{min}$, detection at $273 \mathrm{~nm}): t_{r}($ major $)=8.6 \mathrm{~min}, t_{r}($ minor $)=5.7 \mathrm{~min}, 82: 18$ e.r.

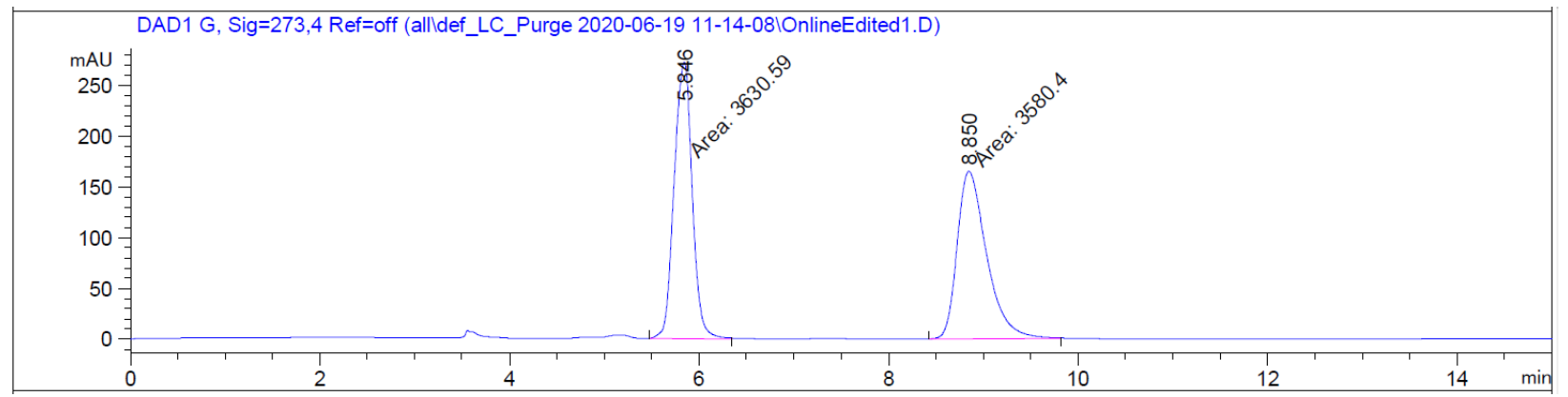



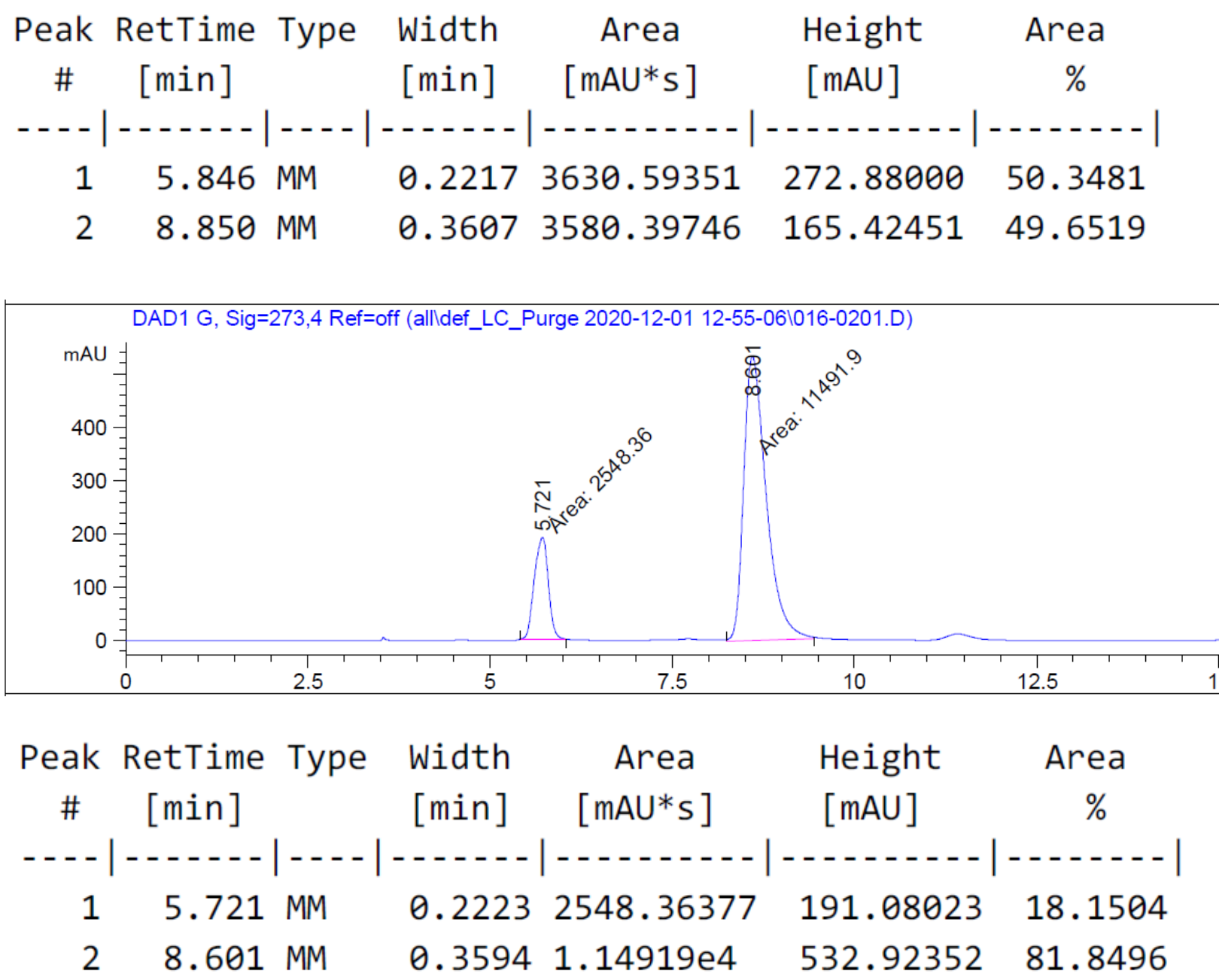

1 mmol scale reaction: A modified procedure A was followed using 5-bromo-1-(5-methylpyridin2-yl)-3-(pent-4-en-1-yl)-1H-indole (1k) (354.0 mg, $1.0 \mathrm{mmol}),\left[\mathrm{RuCl}_{2} \text { (p-cymene) }\right]_{2}$ (30.6 mg, 5.0 mol \%), $\operatorname{AgSbF}_{6}(68.8 \mathrm{mg}, 20 \mathrm{~mol} \%)$ and chiral acid CA8 (100.4 mg, $\left.20 \mathrm{~mol} \%\right)$ in PhMe (2.0 $\mathrm{mL})$ at $25^{\circ} \mathrm{C}$ for $36 \mathrm{~h}$. The reaction mixture was diluted with EtOAc $(5.0 \mathrm{~mL})$ and the solvent was removed in vacuo. The crude mixture was purified by flash column chromatography ( $n$ hexane/EtOAc $=15: 1)$ on silica gel to afford the desired product $2 \mathbf{k}$. $(304.2 \mathrm{mg}, 86 \%)$ as a yellow soild. HPLC separation (Chiralpak ${ }^{\circledR}$ ID-3, $n$-hexane/EtOAc 90:10, $1.0 \mathrm{~mL} / \mathrm{min}$, detection at 273 $\mathrm{nm}): t_{r}($ major $)=8.5 \mathrm{~min}, t_{r}($ minor $)=5.7 \mathrm{~min}, 83: 17$ e.r. 


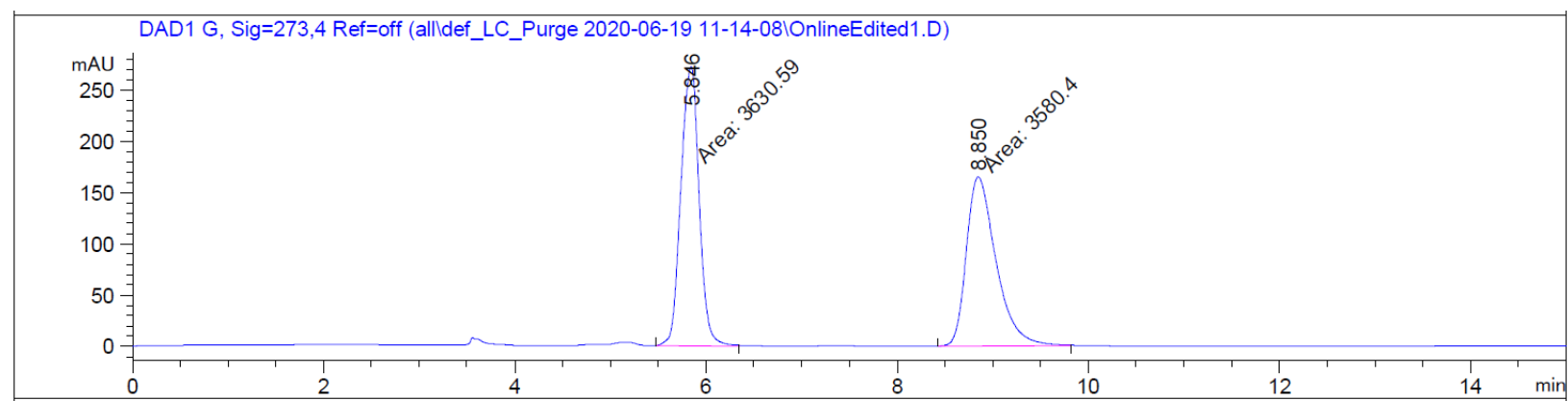

Peak RetTime Type Width Area Height Area

\begin{tabular}{|c|c|c|c|c|c|}
\hline \# & [min] & [min] & {$[\mathrm{mAU} * \mathrm{~s}]$} & [mAU] & $\%$ \\
\hline & & & & & \\
\hline 1 & $5.846 \mathrm{MM}$ & 0.2217 & 3630.59351 & 272.88000 & 50.3481 \\
\hline 2 & $8.850 \mathrm{MM}$ & 0.3607 & 3580.39746 & 165.42451 & 49.6519 \\
\hline
\end{tabular}

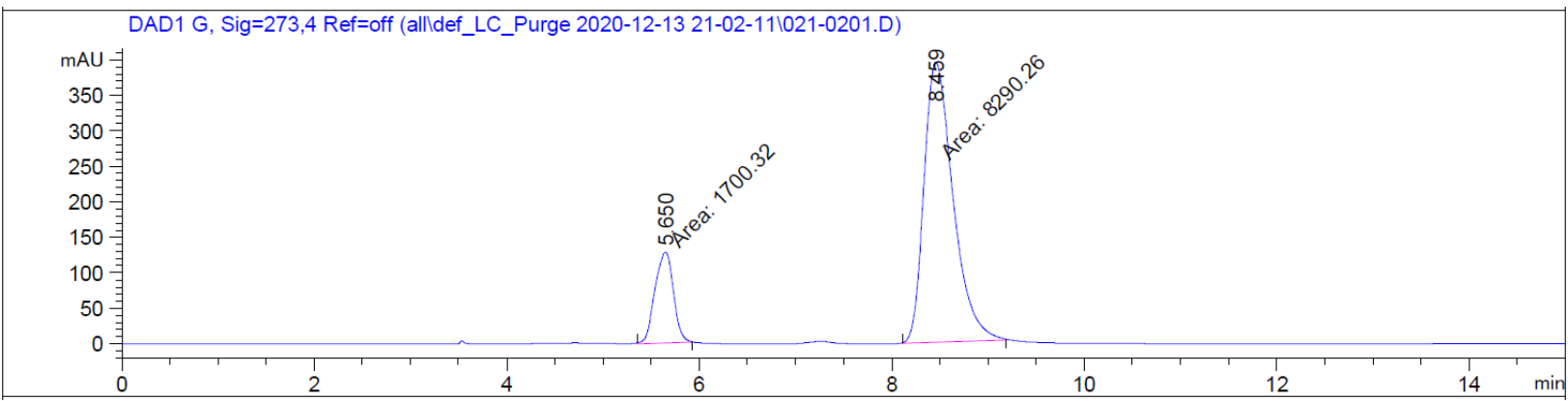

\begin{tabular}{|c|c|c|c|c|c|c|}
\hline $\begin{array}{c}\text { Peak } \\
\text { \# }\end{array}$ & $\begin{array}{c}\text { RetTime } \\
\text { [min] }\end{array}$ & Type & $\begin{array}{l}\text { Width } \\
\text { [min] }\end{array}$ & $\begin{array}{c}\text { Area } \\
{\left[\mathrm{mAU}^{*} \mathrm{~s}\right]}\end{array}$ & $\begin{array}{l}\text { Height } \\
\text { [mAU] }\end{array}$ & $\begin{array}{c}\text { Area } \\
\%\end{array}$ \\
\hline & & & & & & \\
\hline 1 & & & & 1700.3 & 491 & 193 \\
\hline 2 & 8.459 & MM & 504 & 8290.26270 & 394.30869 & 9807 \\
\hline
\end{tabular}




\section{Characterization Data of Products 4}

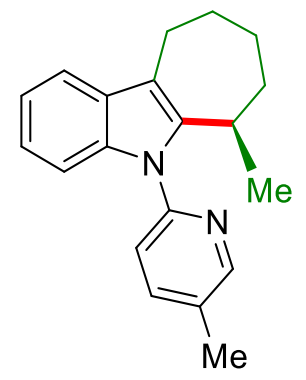

(R)-6-Methyl-5-(5-methylpyridin-2-yl)-5,6,7,8,9,10-hexahydrocyclohepta[b]indole (4a): The general procedure $\mathbf{B}$ was followed using 3-(hex-5-en-1-yl)-1-(5-methylpyridin-2-yl)-1H-indole (3a) $(29.0 \mathrm{mg}, 0.10 \mathrm{mmol})$. Isolation by column chromatography $(n$-hexane/EtOAc $=30: 1)$ yielded 4a $(14.7 \mathrm{mg}, 51 \%)$ as a yellow oil. ${ }^{1} \mathrm{H}-\mathrm{NMR}\left(600 \mathrm{MHz}, \mathrm{CDCl}_{3}\right): \delta=8.45(\mathrm{dd}, J=2.5,0.8$ $\mathrm{Hz}, 1 \mathrm{H}), 7.67-7.61(\mathrm{~m}, 1 \mathrm{H}), 7.51-7.44(\mathrm{~m}, 1 \mathrm{H}), 7.22$ (d, $J=7.0 \mathrm{~Hz}, 1 \mathrm{H}), 7.10$ (dd, $J=8.0,0.8$ $\mathrm{Hz}, 1 \mathrm{H}), 7.07$ (ddd, $J=8.0,7.0,1.2 \mathrm{~Hz}, 1 \mathrm{H}), 7.03$ (ddd, $J=8.0,7.0,1.2 \mathrm{~Hz}, 1 \mathrm{H}), 3.26-3.18$ (m, 1H), 3.06 (ddd, $J=15.4,5.8,2.6 \mathrm{~Hz}, 1 \mathrm{H}), 2.67$ (ddd, $J=15.4,12.4,2.6 \mathrm{~Hz}, 1 \mathrm{H}), 2.40$ (s, 3H), 2.06-1.99 (m, 1H), 1.96-1.87 (m, 2H), 1.84-1.74 (m, 2H), 1.56-1.50 (m, 1H), 1.17 (d, J = 7.2 Hz, $3 \mathrm{H}) .{ }^{13} \mathrm{C}-\mathrm{NMR}\left(100 \mathrm{MHz}, \mathrm{CDCl}_{3}\right): \delta=150.0(\mathrm{CH}), 149.5\left(\mathrm{C}_{\mathrm{q}}\right), 143.1\left(\mathrm{C}_{\mathrm{q}}\right), 138.7(\mathrm{CH}), 136.3\left(\mathrm{C}_{\mathrm{q}}\right)$, $131.8\left(\mathrm{C}_{\mathrm{q}}\right), 128.8\left(\mathrm{C}_{\mathrm{q}}\right), 121.6(\mathrm{CH}), 121.2(\mathrm{CH}), 119.8(\mathrm{CH}), 117.7(\mathrm{CH}), 114.6\left(\mathrm{C}_{\mathrm{q}}\right), 110.0(\mathrm{CH})$, $33.2\left(\mathrm{CH}_{2}\right), 30.5(\mathrm{CH}), 28.7\left(\mathrm{CH}_{2}\right), 25.4\left(\mathrm{CH}_{2}\right), 24.1\left(\mathrm{CH}_{2}\right), 18.4\left(\mathrm{CH}_{3}\right), 18.1\left(\mathrm{CH}_{3}\right)$. IR (ATR): 2920, 1596, 1570, 1483, 1459, 1384, 1201, $738 \mathrm{~cm}^{-1}$. MS (ESI-TOF) $\mathrm{m} / z$ (relative intensity): 313 (10) $[\mathrm{M}+\mathrm{Na}]^{+}, 291(100)[\mathrm{M}+\mathrm{H}]^{+}$. HR-MS (ESI-TOF) $m / z[\mathrm{M}+\mathrm{H}]^{+}$calcd. for $\mathrm{C}_{20} \mathrm{H}_{23} \mathrm{~N}_{2}$ 291.1856; Found 291.1858. $[\alpha]_{\mathrm{D}}^{20}:-7.4\left(\mathrm{c}=1.0, \mathrm{CHCl}_{3}\right)$. HPLC separation (Chiralpak ${ }^{\circledR}$ ID-3, $n$ hexane/EtOAc 90:10, $1.0 \mathrm{~mL} / \mathrm{min}$, detection at $273 \mathrm{~nm}): t_{r}($ major $)=7.6 \mathrm{~min}, t_{r}($ minor $)=6.0 \mathrm{~min}$, $82: 18$ e.r.

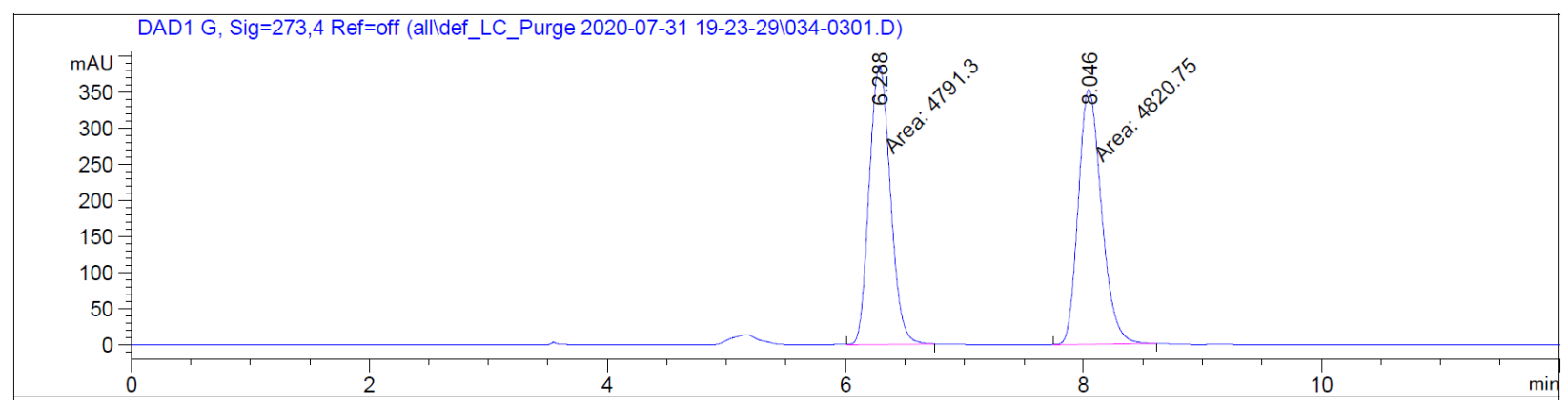


Peak RetTime Type Width Area Height Area

$\begin{array}{lllll}\# & {[\mathrm{~min}]} & {[\mathrm{min}]} & {[\mathrm{mAU} * \mathrm{~s}]} & {[\mathrm{mAU}]}\end{array}$

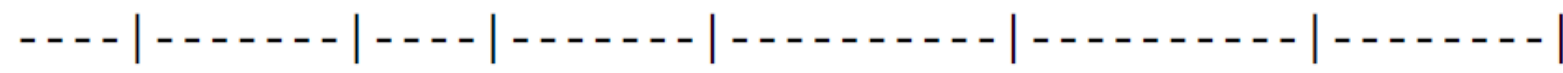

$\begin{array}{llllll}1 & 6.288 \text { MM } & 0.2062 & 4791.29688 & 387.28531 & 49.8468\end{array}$

$\begin{array}{llllll}2 & 8.046 \text { MM } & 0.2268 & 4820.74707 & 354.18680 & 50.1532\end{array}$
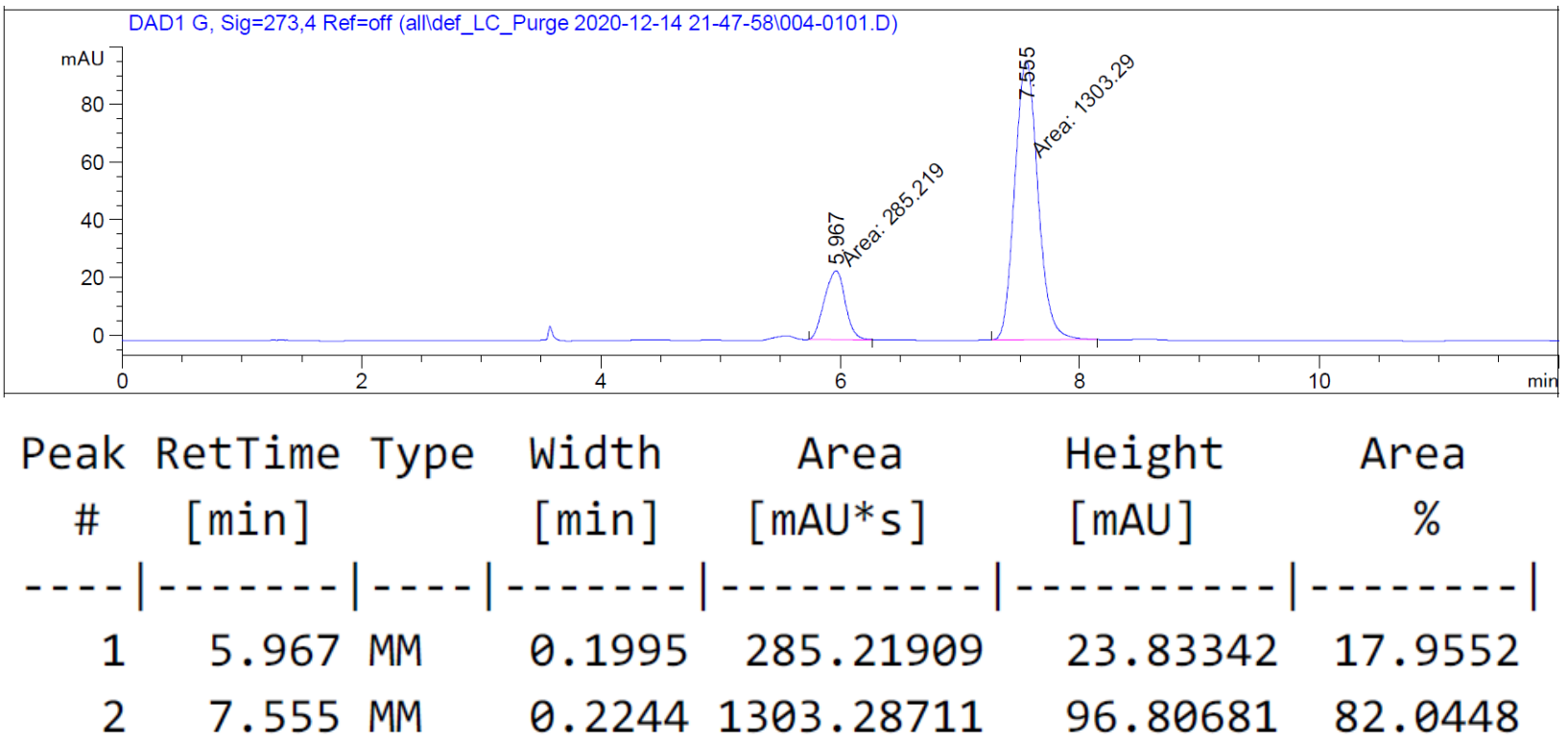<smiles>[M]C1CCCCc2c1n(-c1ccc(C)cn1)c1cc(C)ccc21</smiles>

(R)-2,6-Dimethyl-5-(5-methylpyridin-2-yl)-5,6,7,8,9,10-hexahydrocyclohepta[b]indole (4b):

The general procedure B was followed using 3-(hex-5-en-1-yl)-5-methyl-1-(5-methylpyridin-2yl)- $1 H$-indole (3b) (30.5 mg, $0.10 \mathrm{mmol})$. Isolation by column chromatography ( $n$-hexane/EtOAc $=30: 1)$ yielded $4 \mathbf{b}(14.2 \mathrm{mg}, 47 \%)$ as a yellow oil. ${ }^{1} \mathrm{H}-\mathrm{NMR}\left(600 \mathrm{MHz}, \mathrm{CDCl}_{3}\right): \delta=8.47(\mathrm{dd}, J=$ $2.5,0.8 \mathrm{~Hz}, 1 \mathrm{H}), 7.66(\mathrm{ddd}, J=8.0,2.5,0.8 \mathrm{~Hz}, 1 \mathrm{H}), 7.38-7.35(\mathrm{~m}, 1 \mathrm{H}), 7.23$ (d, $J=8.0 \mathrm{~Hz}, 1 \mathrm{H})$, 6.96-6.87 (m, 2H), 3.20 (qdd, $J=7.2,4.5,3.4 \mathrm{~Hz}, 1 \mathrm{H}), 3.04$ (dddd, $J=15.4,5.7,2.7,0.9 \mathrm{~Hz}, 1 \mathrm{H})$, $2.66(\mathrm{ddd}, J=15.4,12.4,2.7 \mathrm{~Hz}, 1 \mathrm{H}), 2.42$ (s, 3H), 2.35 (s, 3H), 2.06-1.98 (m, 1H), 1.95-1.87 $(\mathrm{m}, 2 \mathrm{H}), 1.85-1.73(\mathrm{~m}, 2 \mathrm{H}), 1.54-1.45(\mathrm{~m}, 1 \mathrm{H}), 1.16(\mathrm{~d}, J=7.2 \mathrm{~Hz}, 3 \mathrm{H}) .{ }^{13} \mathrm{C}-\mathrm{NMR}(100 \mathrm{MHz}$, 
$\left.\mathrm{CDCl}_{3}\right): \delta=149.9(\mathrm{CH}), 149.6\left(\mathrm{C}_{\mathrm{q}}\right), 142.3\left(\mathrm{C}_{\mathrm{q}}\right), 138.6(\mathrm{CH}), 136.7\left(\mathrm{C}_{\mathrm{q}}\right), 131.7\left(\mathrm{C}_{\mathrm{q}}\right), 131.0\left(\mathrm{C}_{\mathrm{q}}\right)$, $126.7\left(\mathrm{C}_{\mathrm{q}}\right), 121.6(\mathrm{CH}), 121.4(\mathrm{CH}), 117.4(\mathrm{CH}), 114.4\left(\mathrm{C}_{\mathrm{q}}\right), 110.0(\mathrm{CH}), 33.3\left(\mathrm{CH}_{2}\right), 30.5(\mathrm{CH})$, $28.7\left(\mathrm{CH}_{2}\right), 25.4\left(\mathrm{CH}_{2}\right), 24.2\left(\mathrm{CH}_{2}\right), 21.7\left(\mathrm{CH}_{3}\right), 18.5\left(\mathrm{CH}_{3}\right), 18.1\left(\mathrm{CH}_{3}\right)$. IR (ATR): 2919, 1571, $1482,1387,1328,839,800,750 \mathrm{~cm}^{-1}$. MS (ESI-TOF) $\mathrm{m} / \mathrm{z}$ (relative intensity): 327 (10) $[\mathrm{M}+\mathrm{Na}]^{+}$, $305(100)[\mathrm{M}+\mathrm{H}]^{+}$. HR-MS (ESI-TOF) $m / z$ : $[\mathrm{M}+\mathrm{Na}]^{+}$Calcd. for $\mathrm{C}_{21} \mathrm{H}_{24} \mathrm{~N}_{2} \mathrm{Na}$ 327.1832; Found 327.1838. $[\alpha]_{\mathrm{D}}^{20}:-8.5\left(\mathrm{c}=1.0, \mathrm{CHCl}_{3}\right)$. HPLC separation $\left(\mathrm{Chiralpak}^{\circledR}\right.$ ID-3, $n$-hexane/EtOAc 90:10, $1.0 \mathrm{~mL} / \mathrm{min}$, detection at $273 \mathrm{~nm}): t_{r}($ major $)=8.6 \mathrm{~min}, t_{r}($ minor $)=6.4 \mathrm{~min}, 84: 16$ e.r.
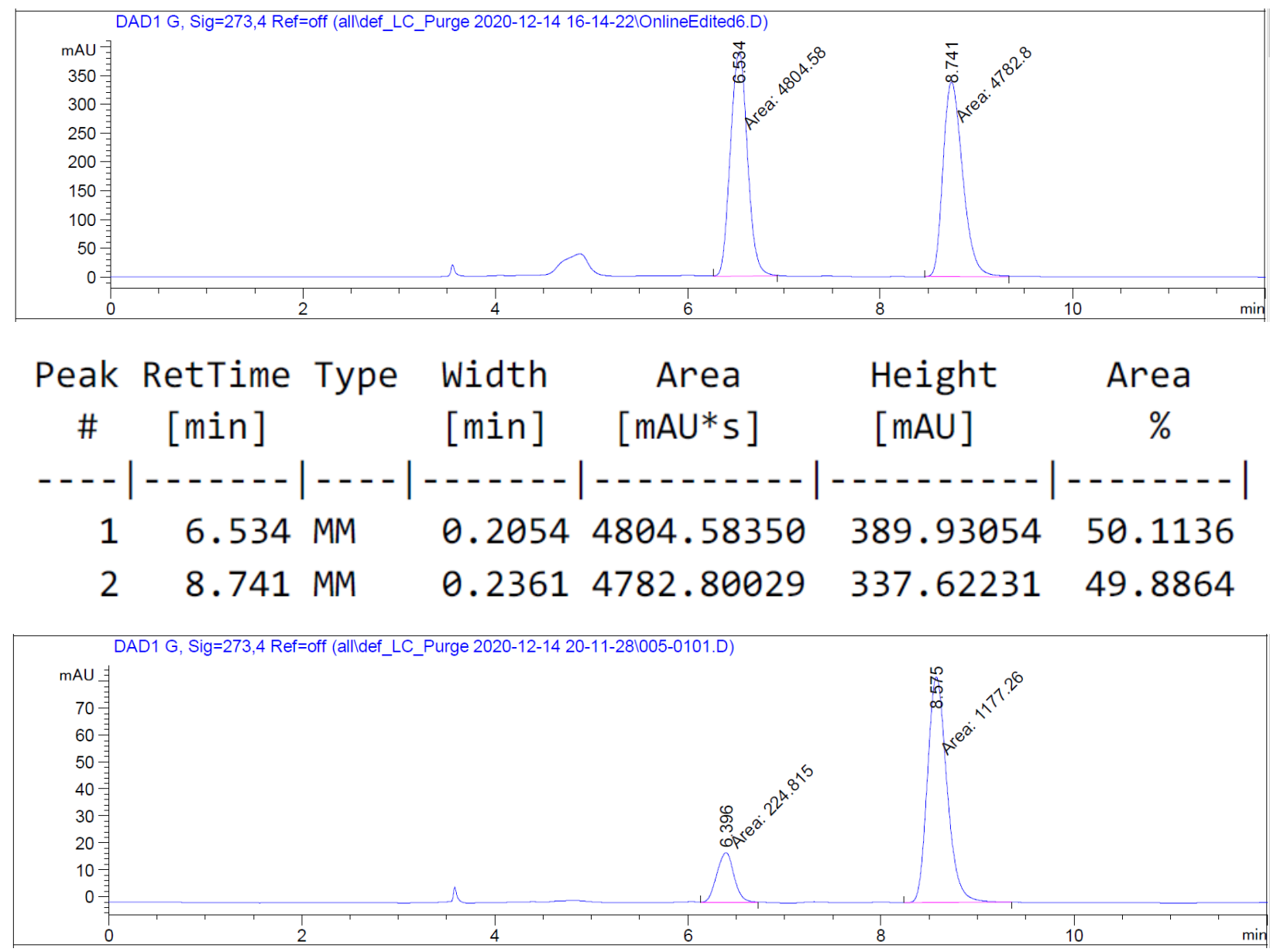

\begin{tabular}{|c|c|c|c|c|c|c|}
\hline $\begin{array}{c}\text { Peak } \\
\text { \# }\end{array}$ & $\begin{array}{c}\text { RetTime } \\
\text { [min] }\end{array}$ & Type & $\begin{array}{l}\text { Width } \\
\text { [min] }\end{array}$ & $\begin{array}{c}\text { Area } \\
{\left[\mathrm{mAU}^{*} \mathrm{~s}\right]}\end{array}$ & $\begin{array}{l}\text { Height } \\
{[\mathrm{mAU}]}\end{array}$ & $\begin{array}{c}\text { Area } \\
\%\end{array}$ \\
\hline & & & & & & \\
\hline 1 & & & & 224.81531 & 25 & \\
\hline & 8.575 & MM & 345 & 1177.2 & 128 & 83 \\
\hline
\end{tabular}




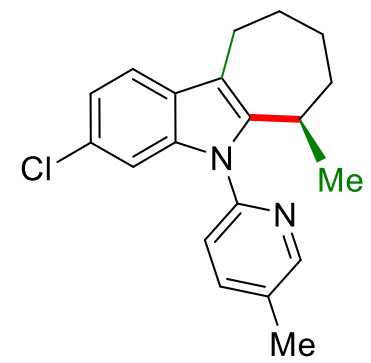

(R)-3-Chloro-6-methyl-5-(5-methylpyridin-2-yl)-5,6,7,8,9,10-hexahydrocyclohepta[b]indole

(4c): The general procedure $\mathbf{B}$ was followed using 6-chloro-3-(hex-5-en-1-yl)-1-(5-methylpyridin2-yl)-1H-indole (3c) $\quad(32.5 \mathrm{mg}, \quad 0.10 \mathrm{mmol})$. Isolation by column chromatography $(n$ hexane/EtOAc $=15: 1)$ yielded $4 \mathbf{c}(19.4 \mathrm{mg}, 60 \%)$ as a white solid. M.p. $142-144{ }^{\circ} \mathrm{C} .{ }^{1} \mathrm{H}-\mathrm{NMR}$ $\left(300 \mathrm{MHz}, \mathrm{CDCl}_{3}\right): \delta=8.51(\mathrm{~d}, J=2.5 \mathrm{~Hz}, 1 \mathrm{H}), 7.72(\mathrm{dd}, J=8.0,2.6 \mathrm{~Hz}, 1 \mathrm{H}), 7.53-7.44(\mathrm{~m}, 1 \mathrm{H})$, 7.32-7.21 (m, 1H), 7.08-7.00 (m, 2H), 3.27-3.18 (m, 1H), 3.10-2.98 (m, 1H), 2.74-2.64 (m, 1H), 2.47 (s, 3H), 2.11-2.03 (m, 1H), 2.00-1.92 (m, 2H), 1.88-1.80 (m, 2H), 1.60-1.45 (m, 1H), 1.23 $(\mathrm{d}, J=7.2 \mathrm{~Hz}, 3 \mathrm{H}) .{ }^{13} \mathrm{C}-\mathrm{NMR}\left(100 \mathrm{MHz}, \mathrm{CDCl}_{3}\right): \delta=149.9(\mathrm{CH}), 148.9\left(\mathrm{C}_{\mathrm{q}}\right), 144.5\left(\mathrm{C}_{\mathrm{q}}\right), 139.0$ $(\mathrm{CH}), 134.7\left(\mathrm{C}_{\mathrm{q}}\right), 132.3\left(\mathrm{C}_{\mathrm{q}}\right), 129.9\left(\mathrm{C}_{\mathrm{q}}\right), 125.6\left(\mathrm{C}_{\mathrm{q}}\right), 121.5(\mathrm{CH}), 121.3(\mathrm{CH}), 117.4(\mathrm{CH}), 114.4$ $\left(\mathrm{C}_{\mathrm{q}}\right), 111.0(\mathrm{CH}), 33.1\left(\mathrm{CH}_{2}\right), 30.6\left(\mathrm{CH}_{3}\right), 28.5\left(\mathrm{CH}_{2}\right), 25.3\left(\mathrm{CH}_{2}\right), 24.1\left(\mathrm{CH}_{2}\right), 18.3\left(\mathrm{CH}_{3}\right), 18.1$ (CH). IR (ATR): 1596, 1571, 1483, 1460, 1445, 1408, 1385, 1062, $793 \mathrm{~cm}^{-1}$. MS (ESI) $\mathrm{m} / z$ (relative intensity): $347.1(11)[\mathrm{M}+\mathrm{Na}]^{+}, 325.2(100)[\mathrm{M}+\mathrm{H}]^{+} \mathrm{HR}-\mathrm{MS}$ (ESI) $\mathrm{m} / \mathrm{z}:[\mathrm{M}+\mathrm{H}]^{+}$ Calcd. for $\mathrm{C}_{20} \mathrm{H}_{22}{ }^{35} \mathrm{ClN}_{2}$ 325.1472; Found: 325.1466. $[\alpha]_{\mathrm{D}}^{20}$ : -8.6 (c = 0.5, $\mathrm{CHCl}_{3}$ ). HPLC separation (Chiralpak ${ }^{\circledR}$ ID-3, $n$-hexane/EtOAc 95:5, $1.0 \mathrm{~mL} / \mathrm{min}$, detection at $273 \mathrm{~nm}$ ): $t_{r}$ (major) $=12.6, t_{r}($ minor $)=8.7 \min , 80: 20$ e.r.

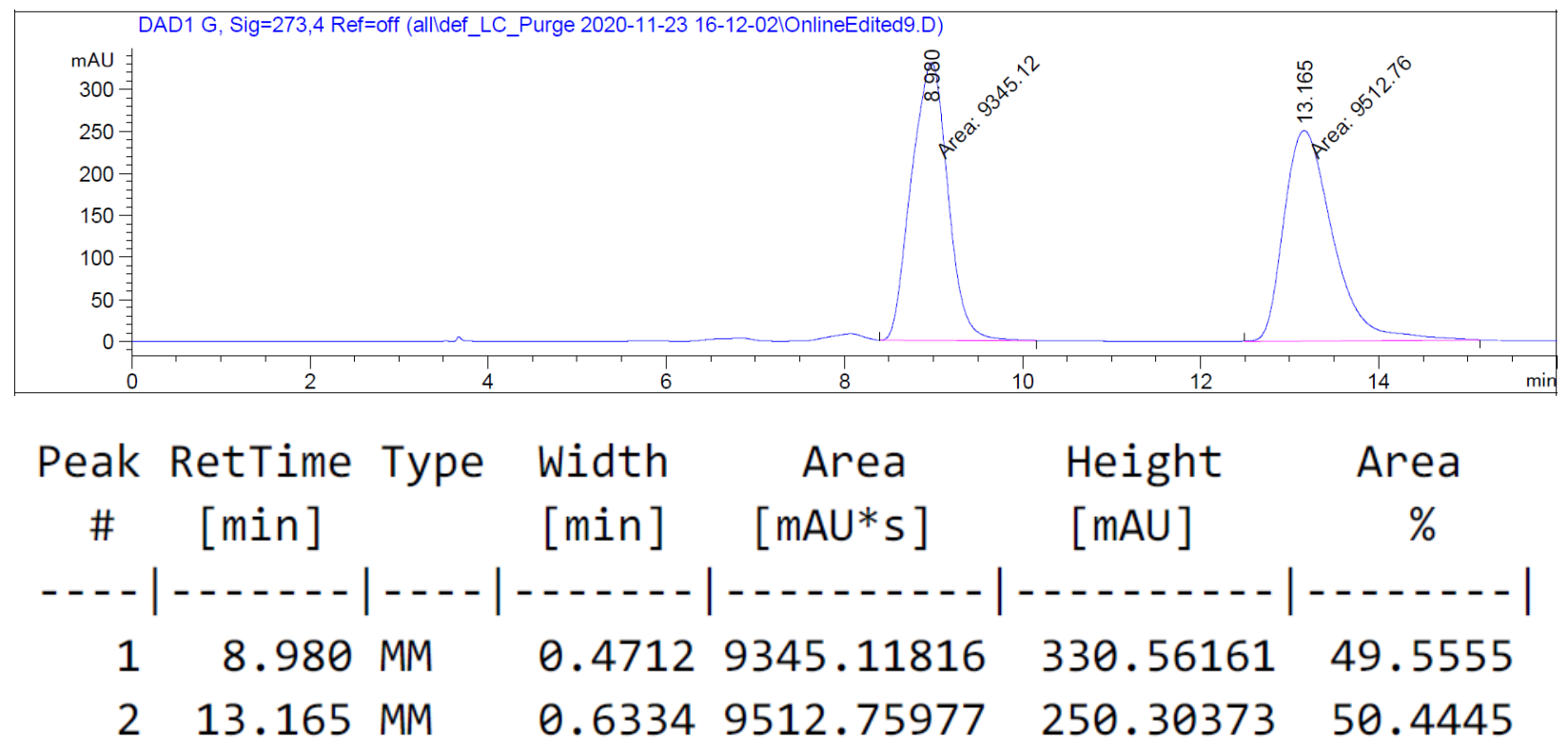



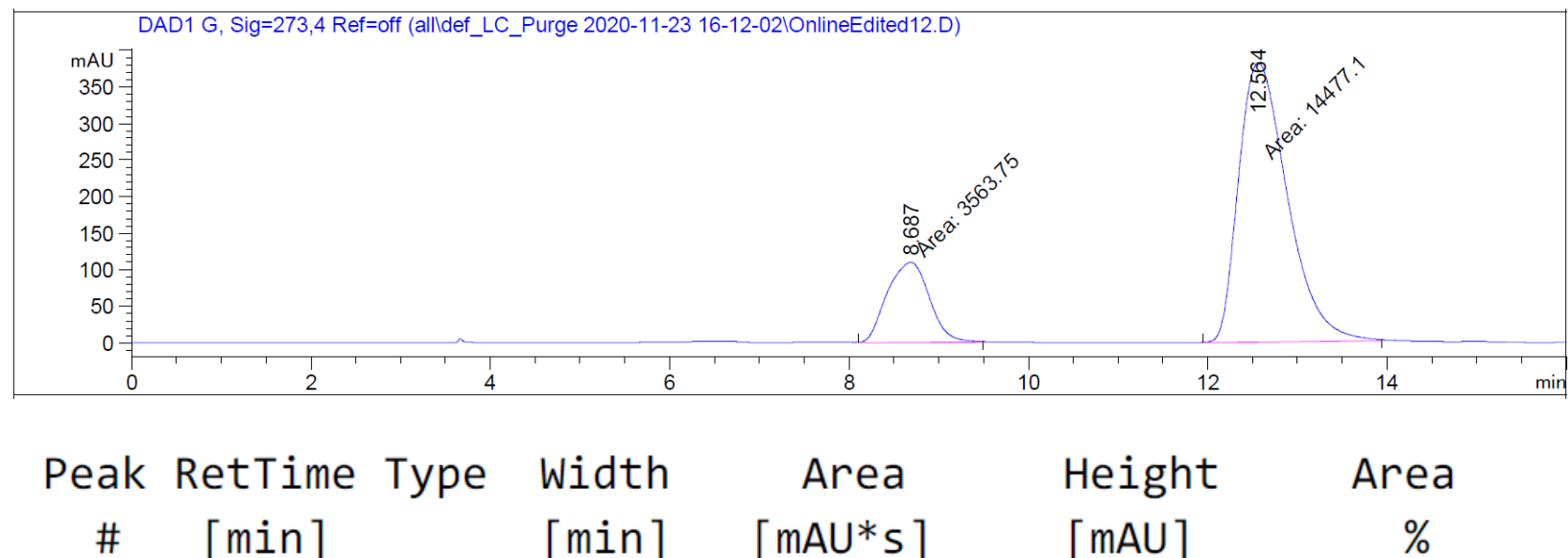

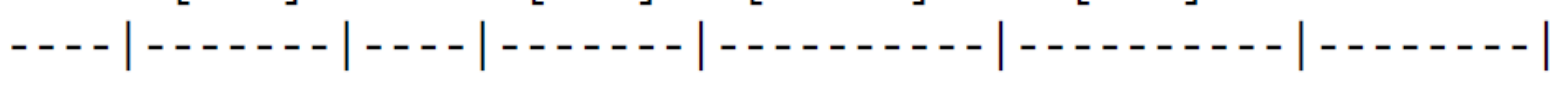

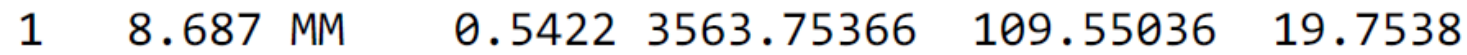

$2 \quad 12.564$ MM $\quad 0.6304 \quad 1.44771 \mathrm{e} 4 \quad 382.76895 \quad 80.2462$ 


\section{Unsuccessful Substrates}

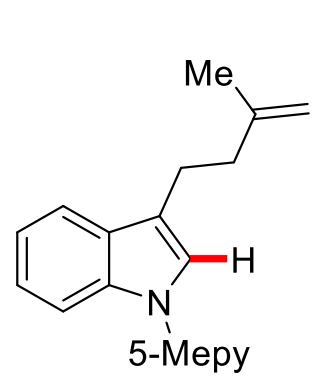

No Reaction

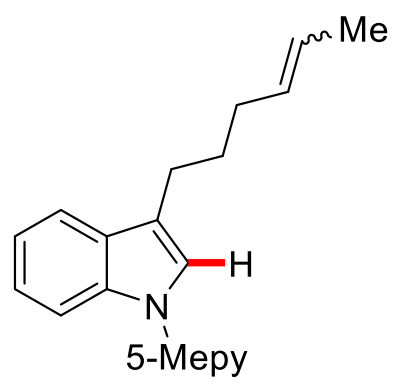

No Reaction

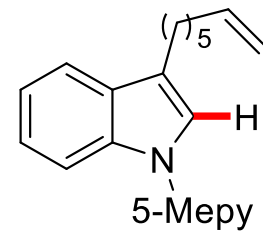

No Reaction<smiles></smiles>

5-Mepy

No Reaction

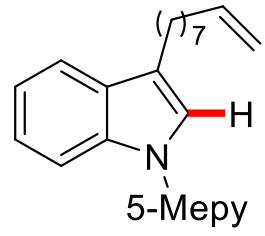

No Reaction 


\section{Mechanistic Studies}

\section{H/D Exchange Experiment}

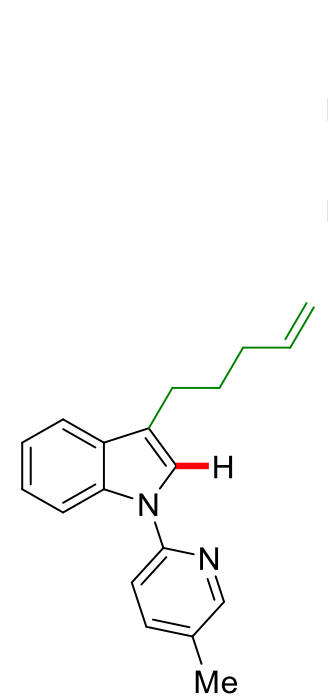

$1 \mathbf{a}$

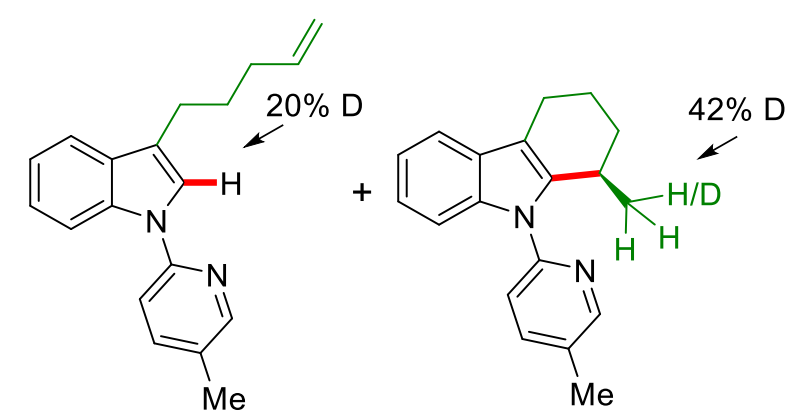

$[\mathrm{D}]_{n}-1 \mathrm{a}: 24 \%$

[D] $]_{n}-2 \mathrm{a}: 71 \%$

The representative procedure A was followed using $1 \mathrm{a}\left(69.0 \mathrm{mg}, 0.25 \mathrm{mmol}, 1\right.$ equiv), $\left[\mathrm{RuCl}_{2}(p\right.$ cymene) $]_{2}(7.7 \mathrm{mg}, 10 \mathrm{~mol} \%), \operatorname{AgSbF}_{6}(17.2 \mathrm{mg}, 20 \mathrm{~mol} \%)$, CA8 (25.2 mg, $\left.20 \mathrm{~mol} \%\right)$ in PhMe $(0.50 \mathrm{~mL})$ and $\mathrm{D}_{2} \mathrm{O}(0.10 \mathrm{~mL})$ at $25^{\circ} \mathrm{C}$ for $12 \mathrm{~h}$. At ambient temperature, the reaction mixture was diluted with EtOAc $(2.0 \mathrm{~mL})$ and the solvents was removed in vacuo. The crude mixture was purified by flash column chromatography on silica gel to afford the desired product $[\mathrm{D}]_{n}-\mathbf{2 a}(48.0$ $\mathrm{mg}, 71 \%)$ and $[\mathrm{D}]_{n}-\mathbf{1 a}(17.0 \mathrm{mg}, 24 \%)$ as yellow oils. 


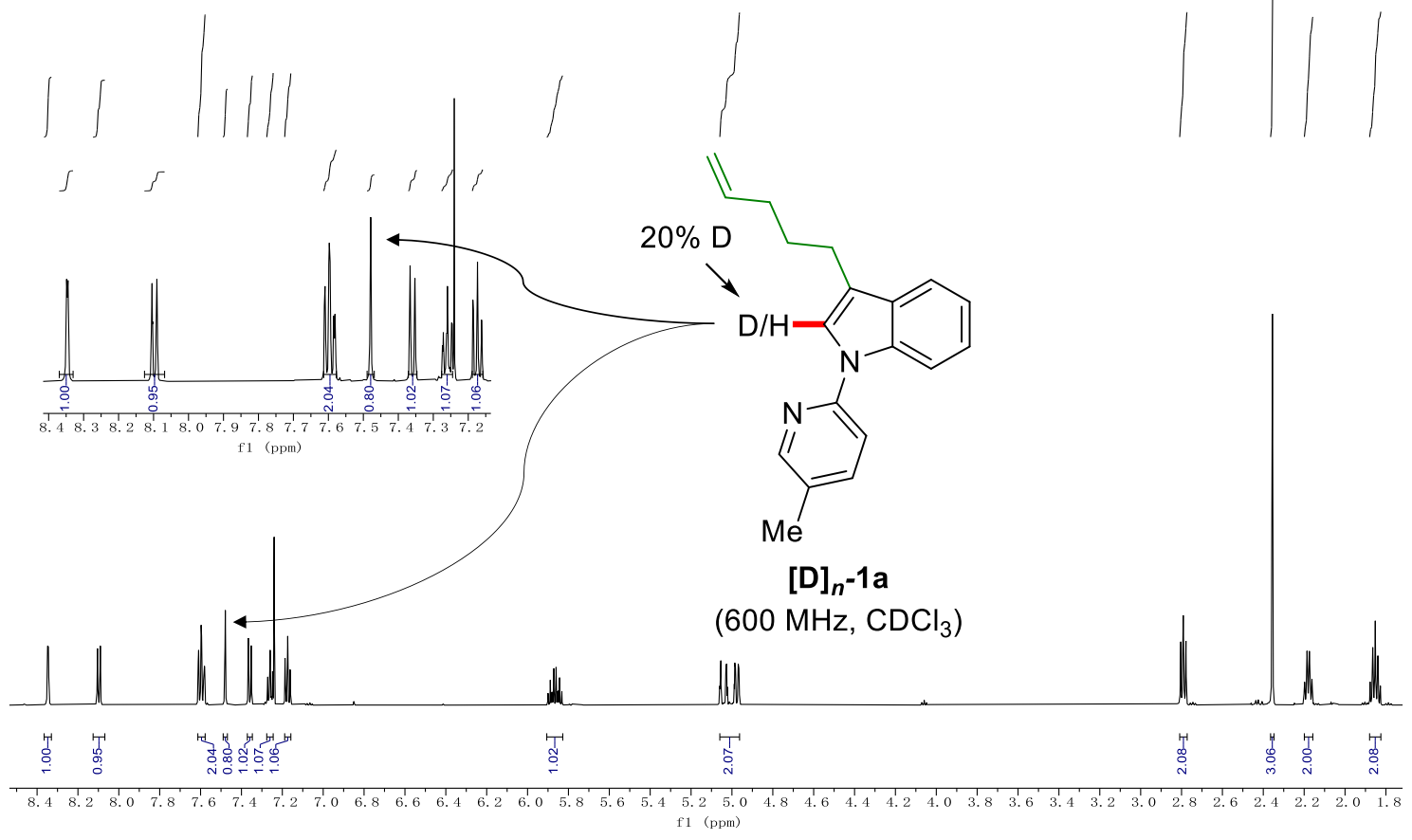

Figure S1. ${ }^{1} \mathrm{H}-\mathrm{NMR}$ of reisolated $[\mathrm{D}]_{n}$-1a from the deuteration study.

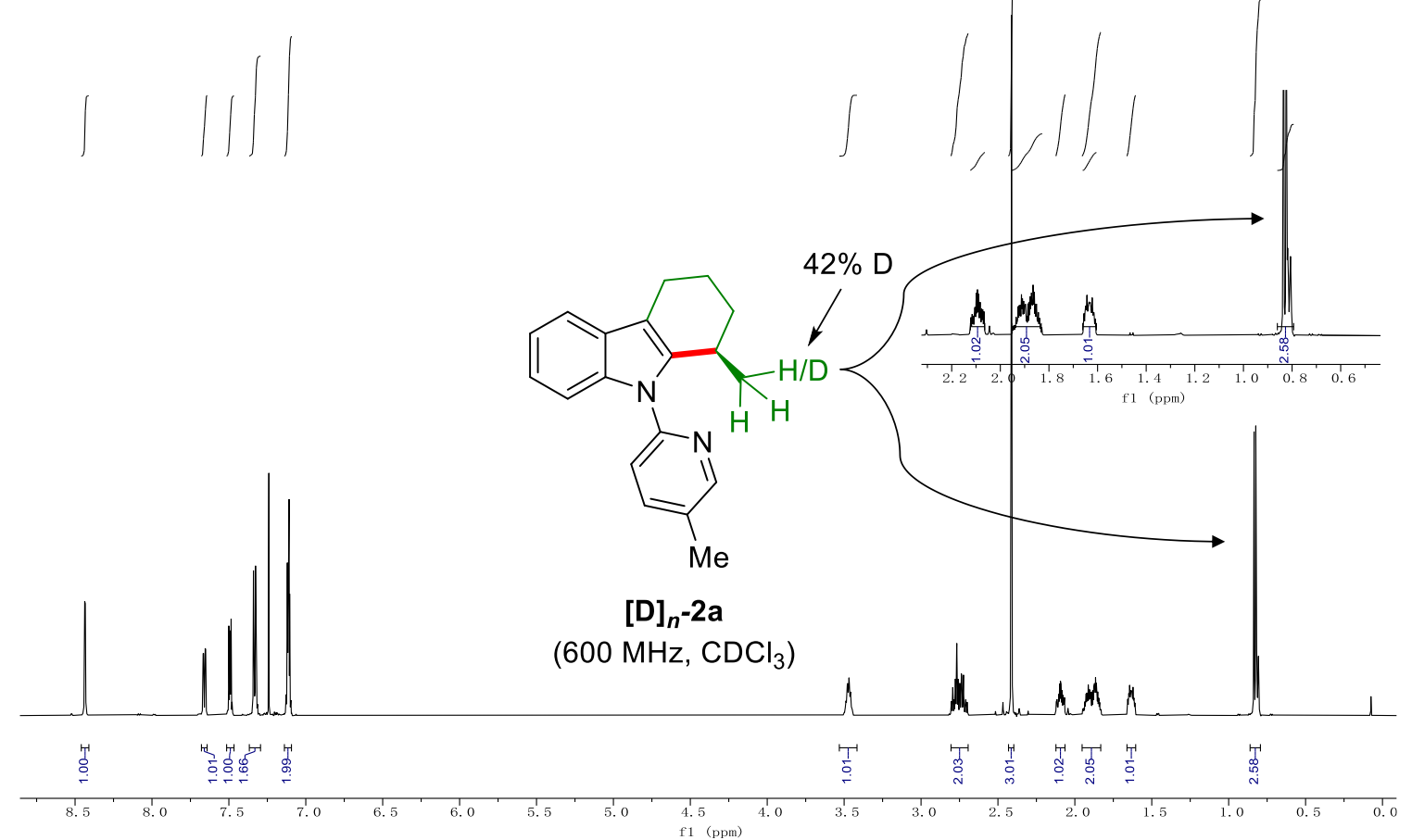

Figure S2. ${ }^{1} \mathrm{H}-\mathrm{NMR}$ of $[\mathrm{D}]_{n}-\mathbf{2 a}$ from the deuteration study. 


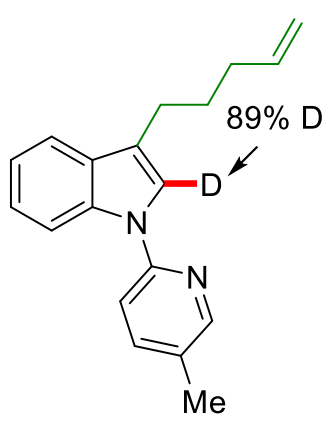

$[\mathrm{D}]_{1}-1 \mathbf{a}$

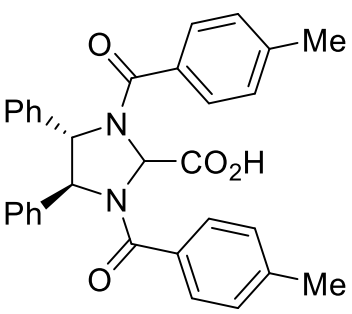

CA8 $(20 \mathrm{~mol} \%)$

$\left[\mathrm{RuCl}_{2}(p \text {-cymene })\right]_{2}$

$\underset{\mathrm{AgSbF}_{6}(20 \mathrm{~mol} \%)}{\stackrel{(5.0 \mathrm{~mol} \%)}{\longrightarrow}}$

PhMe, RT, $2 \mathrm{~h}$

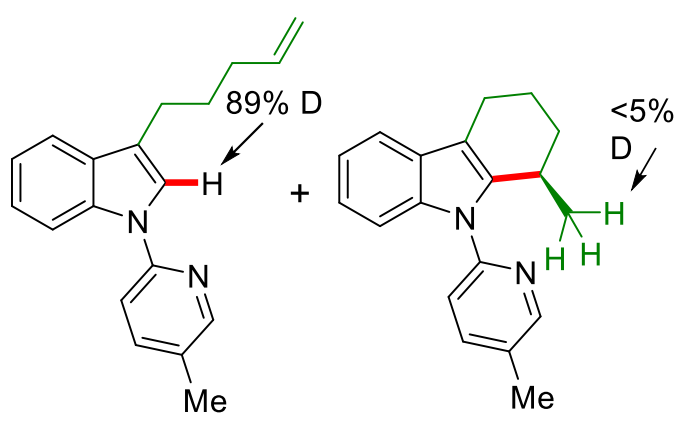

$[D]_{1}-1 \mathrm{a}: 45 \%$

2a: $52 \%$

The representative procedure $\mathbf{A}$ was followed using $[\mathrm{D}]_{1}-\mathbf{1 a}(69.3 \mathrm{mg}, 0.25 \mathrm{mmol}, 1$ equiv), $\left[\mathrm{RuCl}_{2}(p \text {-cymene) }]_{2}(7.7 \mathrm{mg}, 10 \mathrm{~mol} \%), \operatorname{AgSbF}_{6}(17.2 \mathrm{mg}, 20 \mathrm{~mol} \%), \mathbf{C A 8}(25.2 \mathrm{mg}, 20 \mathrm{~mol} \%)\right.$ in $\mathrm{PhMe}(0.50 \mathrm{~mL})$ at $25^{\circ} \mathrm{C}$ for $2 \mathrm{~h}$. At ambient temperature, the reaction mixture was diluted with EtOAc $(2.0 \mathrm{~mL})$ and the solvents was removed in vacuo. The crude mixture was purified by flash column chromatography on silica gel to afford the desired product $\mathbf{2 a}(36.0 \mathrm{mg}, 52 \%)$ and $[\mathrm{D}]_{1}-\mathbf{1 a}$ (31.0 mg, 45\%) as yellow oils. 


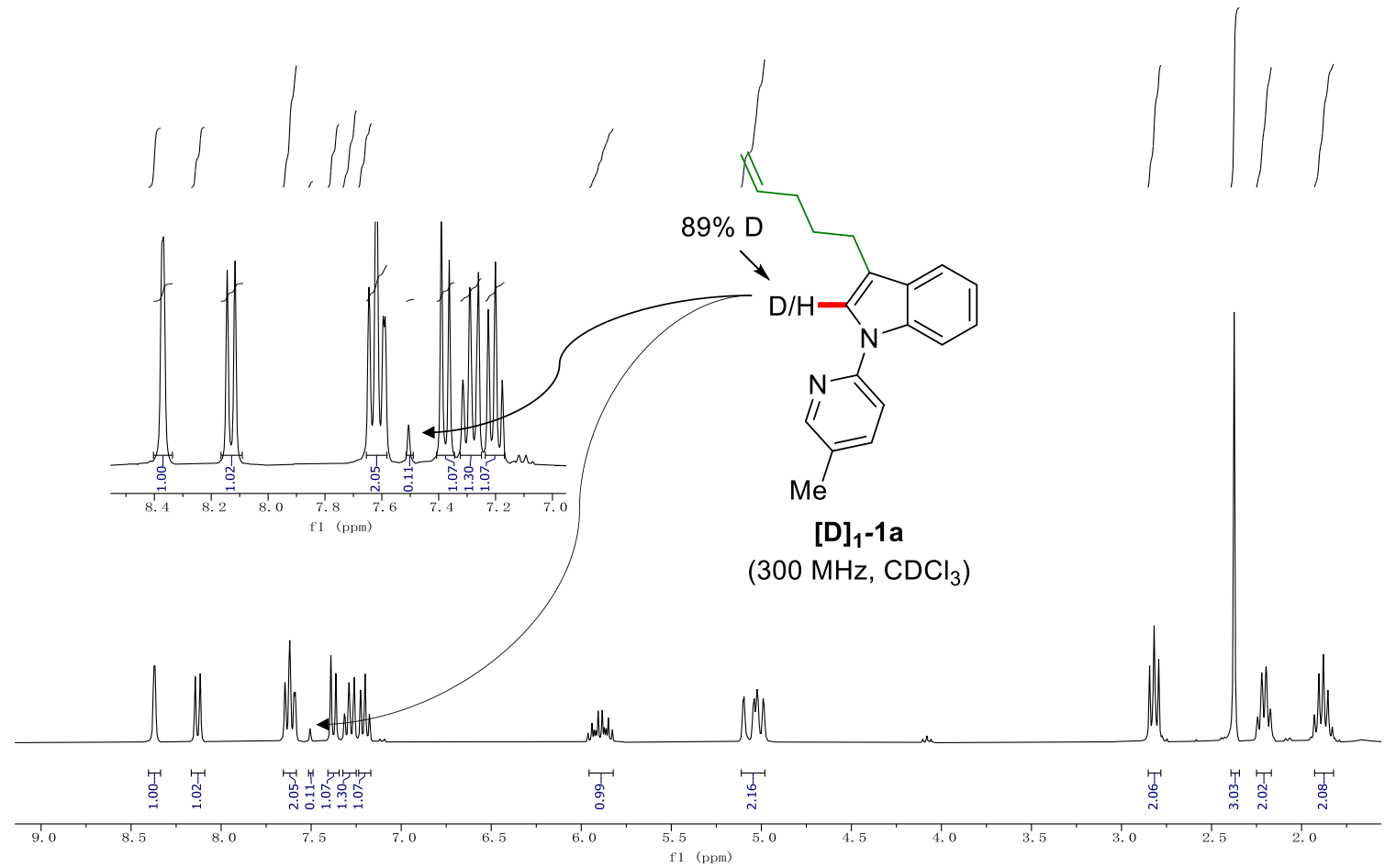

Figure S3. ${ }^{1} \mathrm{H}-\mathrm{NMR}$ of reisolated $[\mathrm{D}]_{1}-\mathbf{1 a}$.

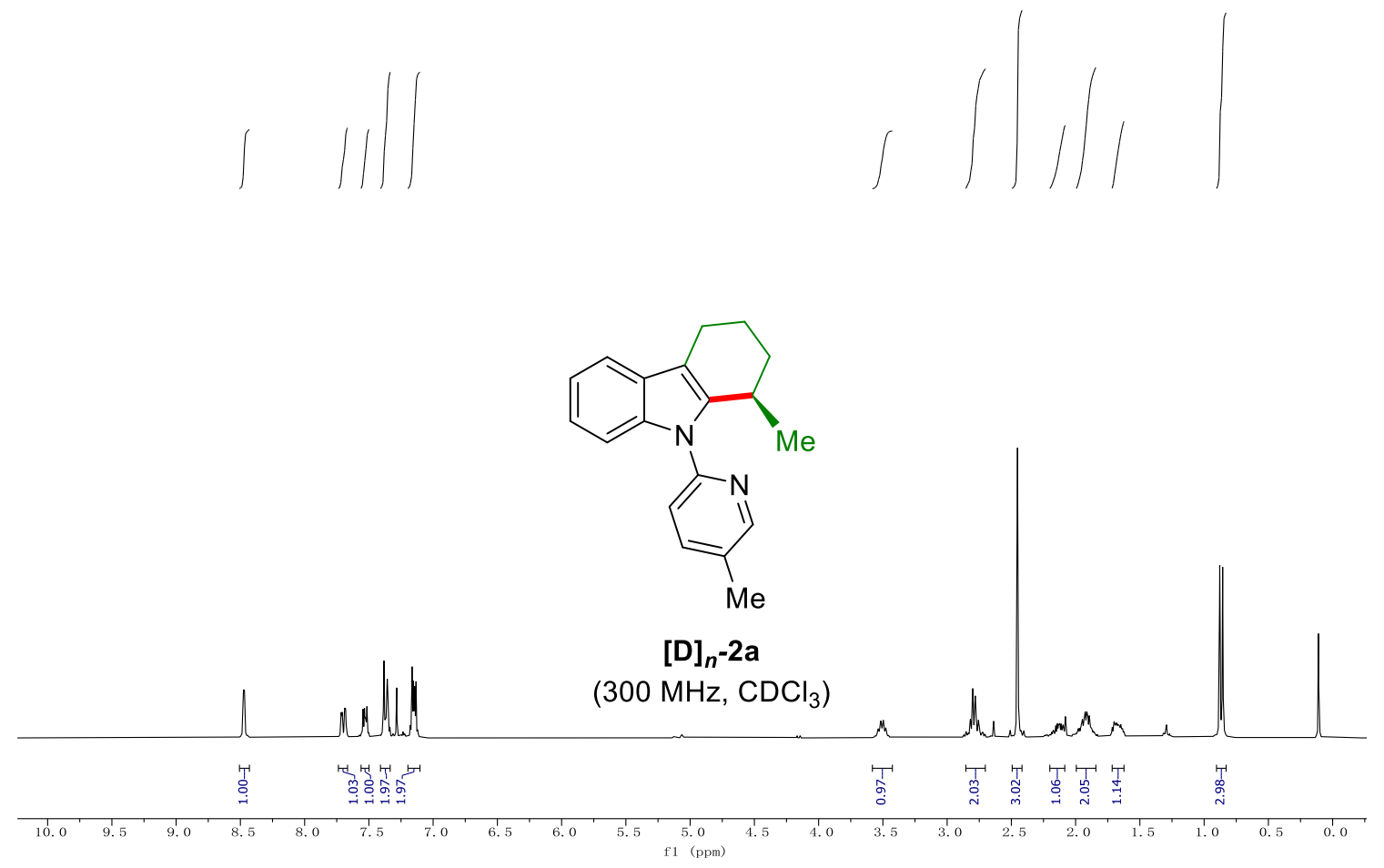

Figure S4. ${ }^{1} \mathrm{H}-\mathrm{NMR}$ of $[\mathrm{D}]_{n}-\mathbf{2 a}$. 


\section{Intramolecular Competition KIE Experiment}

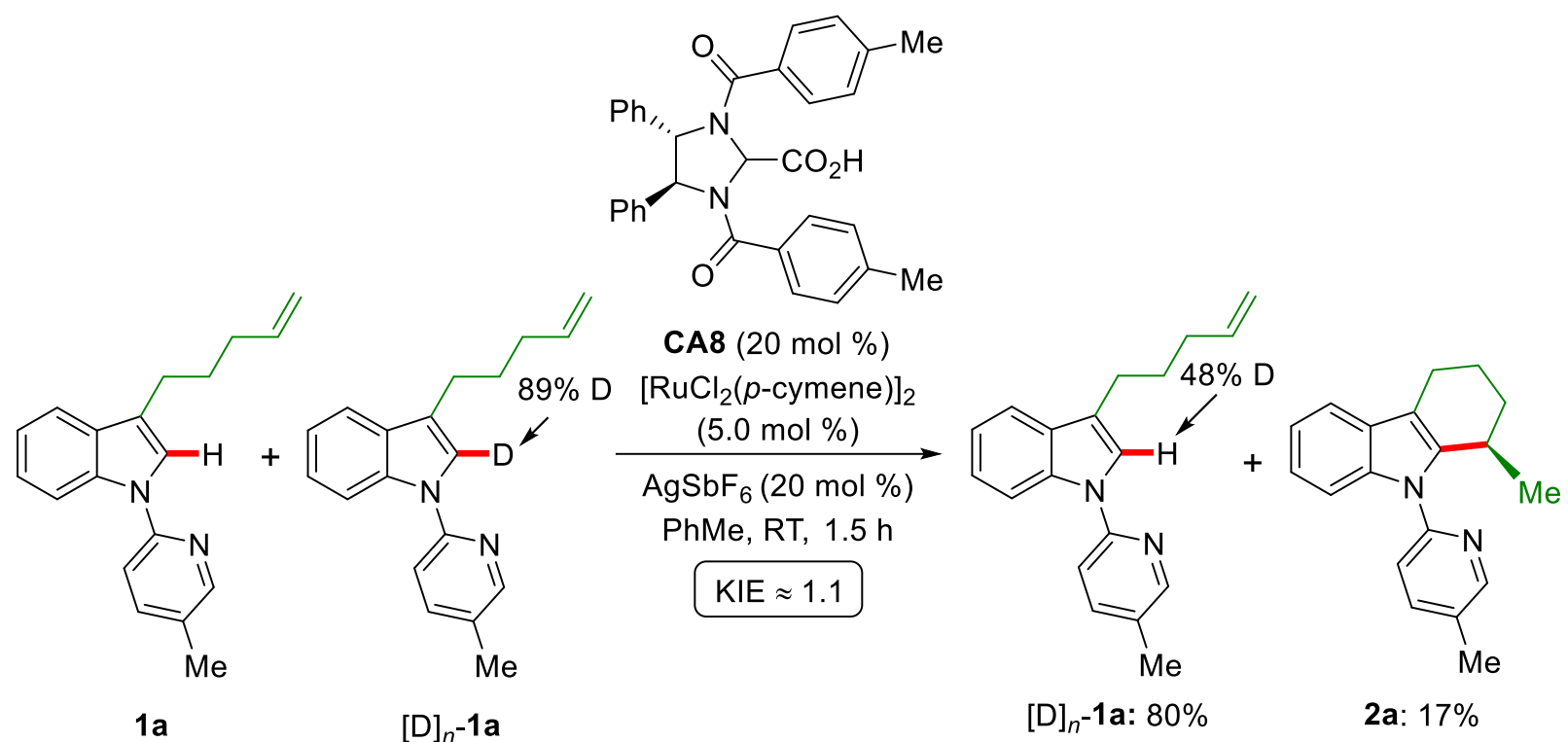

The representative procedure A was followed using $\mathbf{1 a}\left(53.8 \mathrm{mg}, 0.195 \mathrm{mmol}, 1\right.$ equiv), [D] $n^{-1 a}$ (69.3 mg, $0.25 \mathrm{mmol}, 1$ equiv), $\left[\mathrm{RuCl}_{2} \text { (p-cymene) }\right]_{2}$ (13.7 mg, $\left.10 \mathrm{~mol} \%\right), \mathrm{AgSbF}_{6}(30.5 \mathrm{mg}, 20$ mol \%) and CA8 (44.8 mg, $20 \mathrm{~mol} \%)$ in $\mathrm{PhMe}(1.5 \mathrm{~mL})$ at $25^{\circ} \mathrm{C}$ for $1.5 \mathrm{~h}$. The reaction mixture was diluted with EtOAc $(5.0 \mathrm{~mL})$ and the solvent was removed in vacuo. The crude mixture was purified by flash column chromatography on silica gel to afford the desired product $\mathbf{2 a}(21.0 \mathrm{mg}$, $17 \%)$ and $[\mathrm{D}]_{n}-\mathbf{1 a}(98.0 \mathrm{mg}, 80 \%)$ as yellow oils. The kinetic isotope effect of this reaction was determined to be $k_{\mathrm{H}} / k_{\mathrm{D}} \approx 52 / 48 \approx 1.1$ as estimated by ${ }^{1} \mathrm{H}-\mathrm{NMR}$ spectroscopy, based on the recovered starting material. 


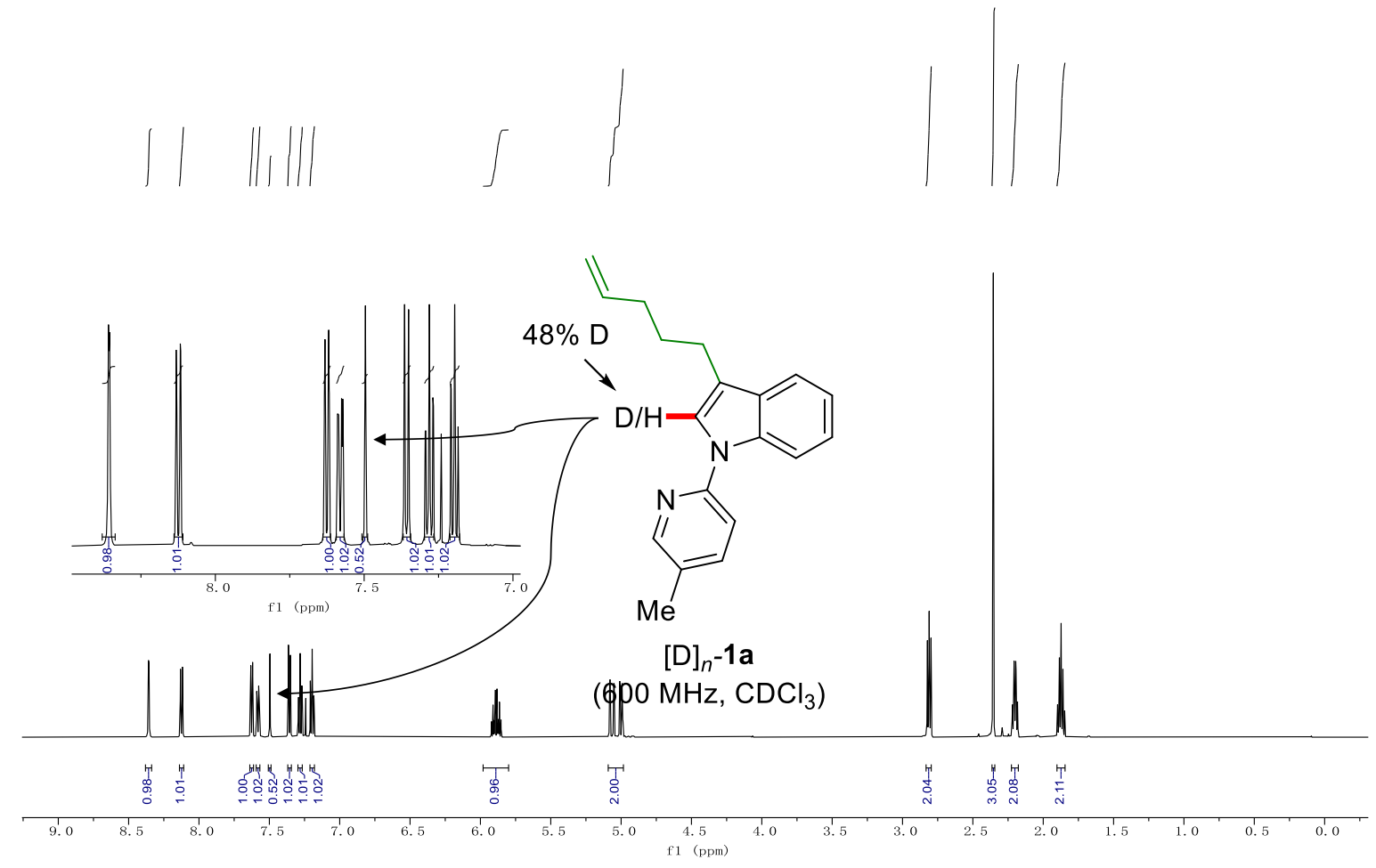

Figure S5. ${ }^{1} \mathrm{H}-\mathrm{NMR}$ of reisolated $[\mathrm{D}]_{n}$-1a from KIE studies.
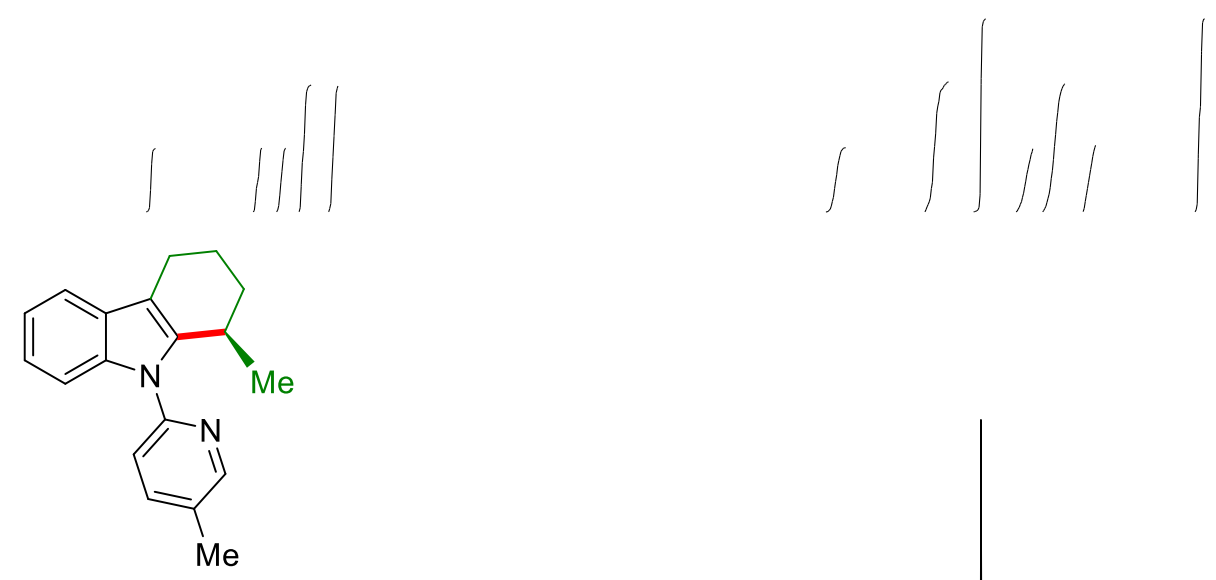

$\stackrel{2 a}{\left(300 \mathrm{MHz}^{-} \mathrm{CDCl}_{3}\right)}$

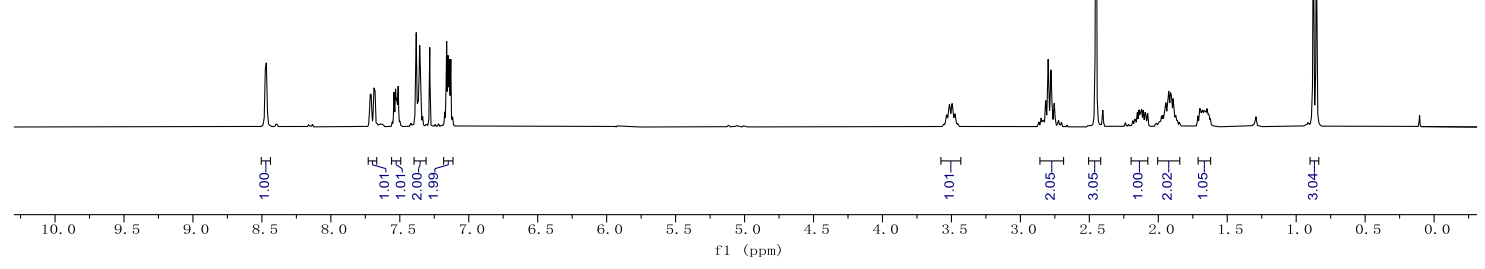

Figure S6. ${ }^{1} \mathrm{H}-\mathrm{NMR}$ of $\mathbf{2 a}$ from KIE studies. 


\section{Determination of Absolute Stereochemistry}

\section{Determination of Absolute Stereochemistry of $2 \mathbf{k}$}

2k (70 mg, 0.20 mmol, 82:18 e.r.) was recrystallized from $\mathrm{CH}_{2} \mathrm{Cl}_{2}$ at $\mathrm{RT}$ by slow evaporation to obtain suitable crystals for X-Ray crystallographic analysis. HPLC separation of crystal 2k (Chiralpak® ID-3, $n$-hexane/EtOAc 90:10, $1.0 \mathrm{~mL} / \mathrm{min}$, detection at $273 \mathrm{~nm}$ ): $t_{r}$ (major) $=8.6 \mathrm{~min}$, $t_{r}($ minor $)=5.7 \mathrm{~min}, 99.4: 0.6$ e.r.
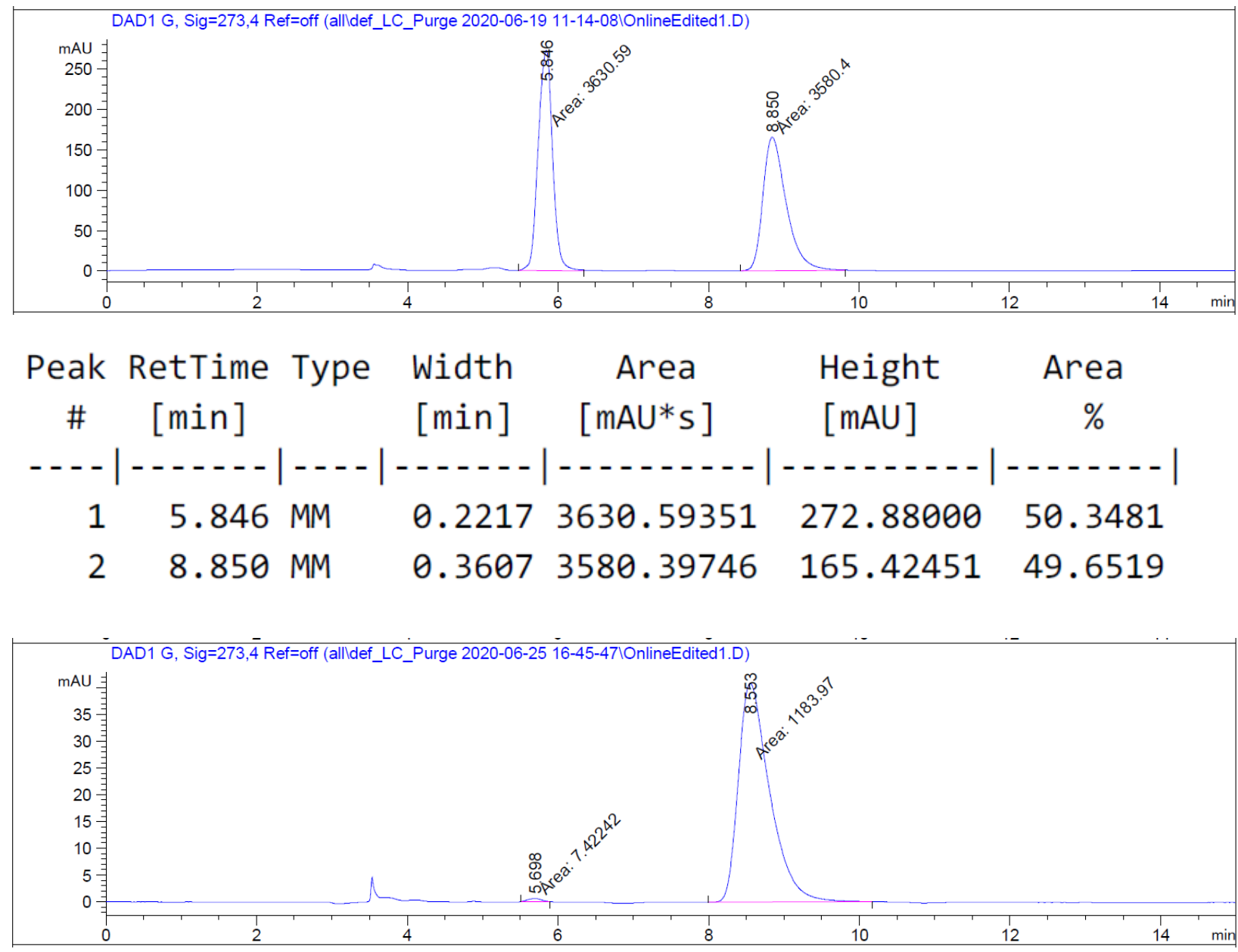

\begin{tabular}{|c|c|c|c|c|c|c|}
\hline $\begin{array}{c}\text { Peak } \\
\#\end{array}$ & $\begin{array}{c}\text { RetTime } \\
\text { [min] }\end{array}$ & Type & $\begin{array}{l}\text { Width } \\
\text { [min] }\end{array}$ & $\begin{array}{c}\text { Area } \\
{\left[\mathrm{mAU}^{*} \mathrm{~s}\right]}\end{array}$ & $\begin{array}{l}\text { Height } \\
{[\mathrm{mAU}]}\end{array}$ & $\begin{array}{c}\text { Area } \\
\%\end{array}$ \\
\hline & & & & - & --- & ------- \\
\hline 1 & 5.698 & MM & 0.2029 & 7.42242 & $6.09674 e-1$ & 0.6230 \\
\hline 2 & 8.553 & MM & 0.4822 & 1183.96558 & 40.92201 & 99.3770 \\
\hline
\end{tabular}




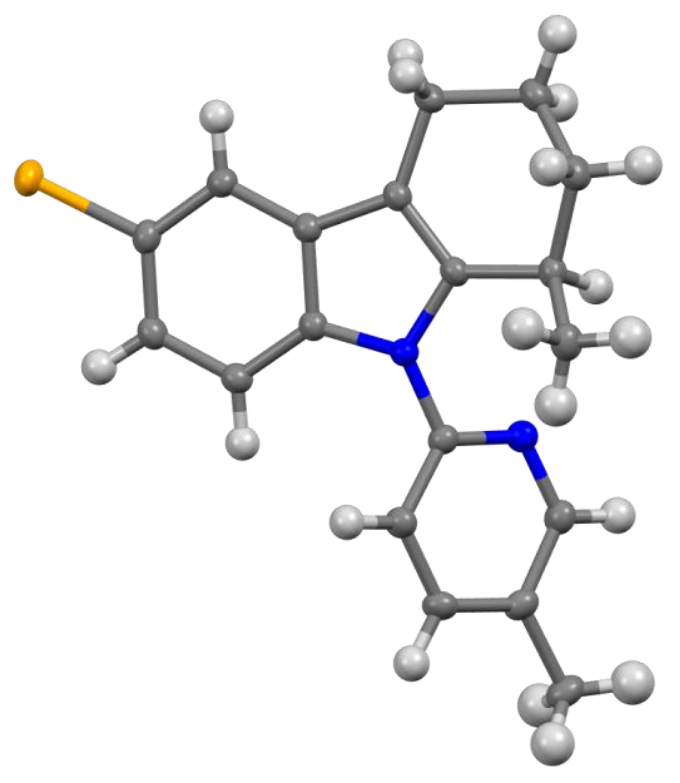

\begin{tabular}{|c|c|}
\hline Compound & $2 \mathbf{k}$ \\
\hline CCDC Number & CCDC 2012314 \\
\hline Empirical formula & $\mathrm{C}_{19} \mathrm{H}_{19} \mathrm{BrN}_{2}$ \\
\hline Formula Weight & 355.27 \\
\hline Temperature & 100.0 \\
\hline Crystal System & orthorhombic \\
\hline Space group & $\mathrm{P} 2{ }_{1} 2_{1} 2_{1}$ \\
\hline $\mathrm{a} / \AA$ & $9.0448(5)$ \\
\hline $\mathrm{b} / \AA$ & $12.6834(6)$ \\
\hline $\mathrm{c} / \AA$ & $13.7386(5)$ \\
\hline$\alpha /^{\circ}$ & 90 \\
\hline$\beta /^{\circ}$ & 90 \\
\hline$\gamma / /^{\circ}$ & 90 \\
\hline Volume $/ \AA^{3}$ & $1576.08(13)$ \\
\hline $\mathrm{Z}$ & 4 \\
\hline$\rho_{\text {calc }} g / \mathrm{cm}^{3}$ & 1.497 \\
\hline$\mu / \mathrm{mm}^{-1}$ & 2.606 \\
\hline $\mathrm{F}(000)$ & 728.0 \\
\hline Crystal size $/ \mathrm{mm}^{3}$ & $0.344 \times 0.172 \times 0.155$ \\
\hline
\end{tabular}




\begin{tabular}{|l|c|}
\hline Radiation & $\operatorname{MoK} \alpha(\lambda=0.71073)$ \\
\hline $2 \Theta$ range for data collection ${ }^{\circ}$ & 4.37 to 61.036 \\
\hline Index ranges & 54097 \\
\hline Reflections collected & $-12 \leq \mathrm{h} \leq 12,-18 \leq \mathrm{k} \leq 18,-19 \leq 1 \leq 18$ \\
\hline Independent reflections & $4808\left[\mathrm{R}_{\text {int }}=0.0218, \mathrm{R}_{\text {sigma }}=0.0107\right]$ \\
\hline Data/restraints/parameters & $1.067 / 0 / 201$ \\
\hline Goodness-of-fit on $\mathrm{F}^{2}$ & $\mathrm{R}_{1}=0.0188, \mathrm{wR}_{2}=0.0501$ \\
\hline Final $\mathrm{R}$ indexes $[\mathrm{I}>=2 \sigma(\mathrm{I})]$ & $\mathrm{R}_{1}=0.0194, \mathrm{wR}_{2}=0.0509$ \\
\hline Final $\mathrm{R}$ indexes [all data] & $0.55 /-0.19$ \\
\hline Largest diff. peak/hole / e $\AA^{-3}$ & $0.0083(14)$ \\
\hline Flack parameter & \\
\hline
\end{tabular}

Table S3: Selected bond lengths $[\AA ̊ \AA]$ and angles $\left[{ }^{\circ}\right]$ for $2 k$

$\begin{array}{cccc}\text { Br1-C1 } & 1.9134(16) & \text { C6-C1-Br1 } & 118.57(12) \\ \text { N1-C4 } & 1.397(2) & \text { C6-C1-C2 } & 123.22(15) \\ \text { N1-C8 } & 1.410(2) & \text { C3-C2-C1 } & 119.93(15) \\ \text { N1-C14 } & 1.416(2) & \text { C2-C3-C4 } & 118.00(15) \\ \text { N2-C14 } & 1.336(2) & \text { N1-C4-C5 } & 107.50(14) \\ \text { N2-C18 } & 1.335(2) & \text { C3-C4-N1 } & 130.62(15) \\ \text { C1-C2 } & 1.397(2) & \text { C3-C4-C5 } & 121.88(15) \\ \text { C1-C6 } & 1.383(2) & \text { C4-C5-C7 } & 107.48(14) \\ \text { C2-C3 } & 1.388(2) & \text { C6-C5-C4 } & 119.67(15) \\ \text { C3-C4 } & 1.393(2) & \text { C6-C5-C7 } & 132.84(15) \\ \text { C4-C5 } & 1.416(2) & \text { C1-C6-C5 } & 117.24(15) \\ \text { C5-C6 } & 1.406(2) & \text { C5-C7-C12 } & 128.90(14) \\ \text { C5-C7 } & 1.430(2) & \text { C8-C7-C5 } & 107.64(14) \\ \text { C7-C8 } & 1.366(2) & \text { C8-C7-C12 } & 123.39(14) \\ \text { C7-C12 } & 1.501(2) & \text { N1-C8-C9 } & 124.30(14) \\ \text { C8-C9 } & 1.499(2) & \text { C7-C8-N1 } & 109.45(14) \\ \text { C9-C10 } & 1.558(2) & \text { C7-C8-C9 } & 125.96(15)\end{array}$




$\begin{array}{cccc}\text { C9-C13 } & 1.530(2) & \mathrm{C} 8-\mathrm{C} 9-\mathrm{C} 10 & 107.93(14) \\ \mathrm{C} 10-\mathrm{C} 11 & 1.526(2) & \mathrm{C} 8-\mathrm{C} 9-\mathrm{C} 13 & 111.97(14) \\ \mathrm{C} 11-\mathrm{C} 12 & 1.528(2) & \mathrm{C} 13-\mathrm{C} 9-\mathrm{C} 10 & 108.24(14) \\ \mathrm{C} 14-\mathrm{C} 15 & 1.392(2) & \mathrm{C} 11-\mathrm{C} 10-\mathrm{C} 9 & 112.86(15) \\ \mathrm{C} 15-\mathrm{C} 16 & 1.390(2) & \mathrm{C} 10-\mathrm{C} 11-\mathrm{C} 12 & 110.35(15) \\ \mathrm{C} 16-\mathrm{C} 17 & 1.393(2) & \mathrm{C} 7-\mathrm{C} 12-\mathrm{C} 11 & 109.53(14) \\ \mathrm{C} 17-\mathrm{C} 18 & 1.397(2) & \mathrm{N} 2-\mathrm{C} 14-\mathrm{N} 1 & 115.55(14) \\ \mathrm{C} 17-\mathrm{C} 19 & 1.505(2) & \mathrm{N} 2-\mathrm{C} 14-\mathrm{C} 15 & 123.07(16) \\ \mathrm{C} 4-\mathrm{N} 1-\mathrm{C} 8 & 107.92(13) & \mathrm{C} 15-\mathrm{C} 14-\mathrm{N} 1 & 121.38(15) \\ \mathrm{C} 4-\mathrm{N} 1-\mathrm{C} 14 & 124.61(14) & \mathrm{C} 16-\mathrm{C} 15-\mathrm{C} 14 & 118.31(16) \\ \mathrm{C} 8-\mathrm{N} 1-\mathrm{C} 14 & 125.71(14) & \mathrm{C} 15-\mathrm{C} 16-\mathrm{C} 17 & 119.87(16) \\ \mathrm{C} 18-\mathrm{N} 2-\mathrm{C} 14 & 117.42(15) & \mathrm{C} 16-\mathrm{C} 17-\mathrm{C} 18 & 116.58(16) \\ \mathrm{C} 2-\mathrm{C} 1-\mathrm{Br} 1 & 118.21(12) & \mathrm{C} 16-\mathrm{C} 17-\mathrm{C} 19 & 122.65(17) \\ & & \mathrm{C} 18-\mathrm{C} 17-\mathrm{C} 19 & 120.76(16) \\ & & \mathrm{N} 2-\mathrm{C} 18-\mathrm{C} 17 & 124.62(16)\end{array}$




\section{Computational Studies}

All calculations were performed using the Gaussian 16, Revision A.03 package. ${ }^{6}$ All structures were optimized at the $\mathrm{PBE}^{7}$ level of theory in combination with $\mathrm{D} 3$ dispersion corrections with the Becke-Johnson damping scheme (D3BJ). ${ }^{8}$ Analytical frequency calculations were carried out at the same level of theory in order to identify each stationary point as either an intermediate (no imaginary frequencies) or a transition state (only one imaginary frequency) and to provided thermal and non-thermal corrections to the Gibbs free energy at $298.15 \mathrm{~K}$ and $1 \mathrm{~atm}$. All atoms were described with the def2-SVP basis set, ${ }^{9}$ while ruthenium was in addition described with a SSD pseudopotential. ${ }^{10}$ The electronic energy was then refined through PW6B95 ${ }^{11}$ single-point calculations on the optimized geometries in combination with a standalone version of Grimme's most recent dispersion correction $\mathrm{D} 4^{12}$ with a def2-TZVP basis set $^{9}$ combined with a SSD pseudopotential for ruthenium. ${ }^{10}$ Solvent effects were included implicitly through the use of the SMD model ${ }^{13}$ for toluene. Energies reported herein are based on gas-phase Gibbs free energies with a def2-SVP basis set for which the electronic energies were corrected to PW6B95-D4 or PBE0-D4 with a def2-TZVP basis set and solvent effects. Non-covalent interactions were localized and visualized through the use of the NCIPLOT program. ${ }^{14}$ 


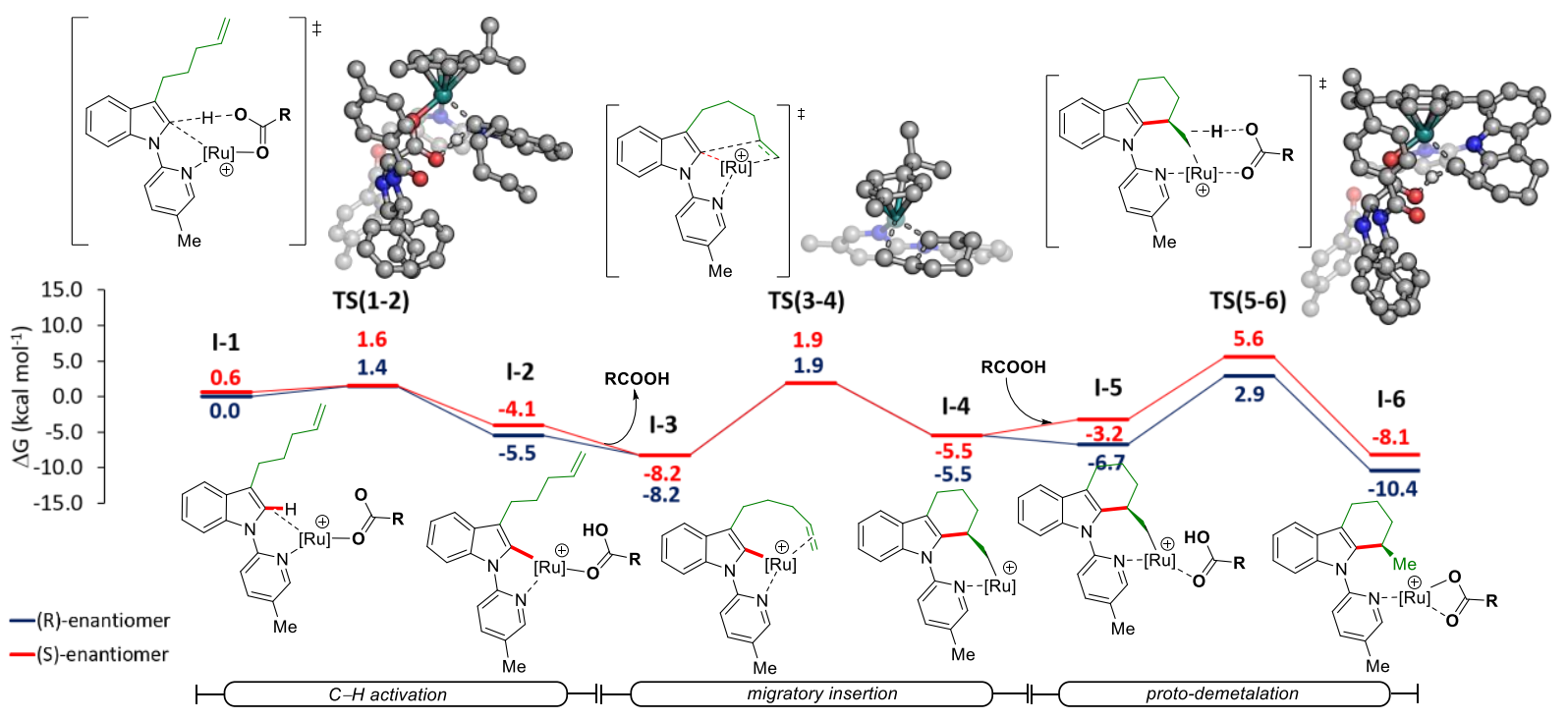

Figure S7. Computed relative Gibbs free energies $\left(\Delta \mathrm{G}_{298.15}\right)$ in $\mathrm{kcal} \mathrm{mol}^{-1}$ between the $\mathrm{C}-\mathrm{H}$ activation and proto-demetalation elementary steps at the PBE0-D4/def2-TZVPSMD(Toluene)//PBE0-D3BJ/def2-SVP level of theory for both enantiomers. In the computed transition state structures, non-relevant hydrogens were omitted for clarity. With $[\mathrm{Ru}]=\mathrm{Ru}(p$ cymene) and $\mathrm{RCO}_{2} \mathrm{H}$ stands for the chiral acid CA8. For legibility, only the structures for the formation of the $(R)$-enantiomer pathway are shown.

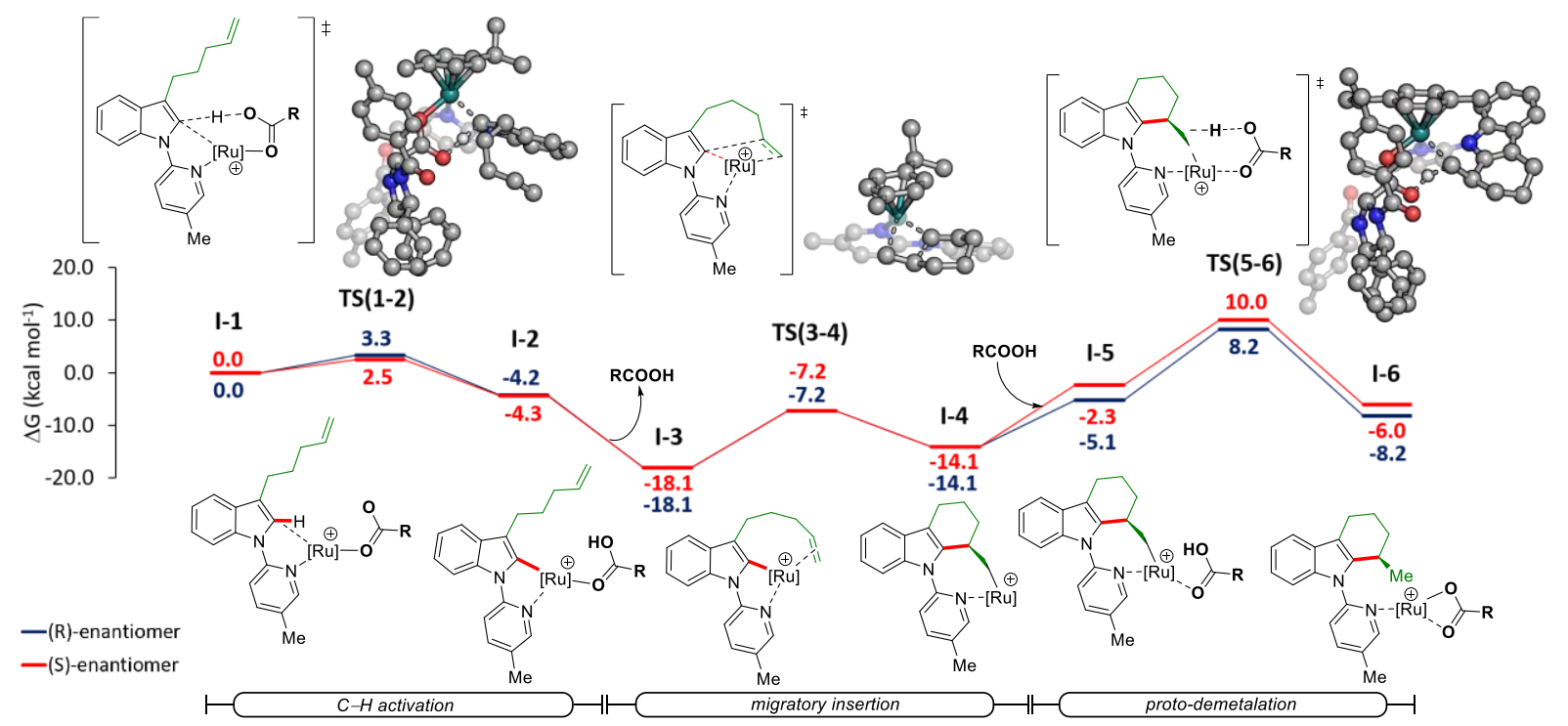

Figure S8. Computed relative Gibbs free energies $\left(\Delta \mathrm{G}_{298.15}\right)$ in $\mathrm{kcal} \mathrm{mol}^{-1}$ between the $\mathrm{C}-\mathrm{H}$ activation and proto-demetalation elementary steps at the PW6B95/def2-TZVPSMD(Toluene)//PBE0-D3BJ/def2-SVP level of theory for both enantiomers. In the computed transition state structures, non-relevant hydrogens were omitted for clarity. With $[\mathrm{Ru}]=\mathrm{Ru}(p$ cymene) and $\mathrm{RCO}_{2} \mathrm{H}$ stands for the chiral acid CA8. For legibility, only the structures for the formation of the $(R)$-enantiomer pathway are shown. 


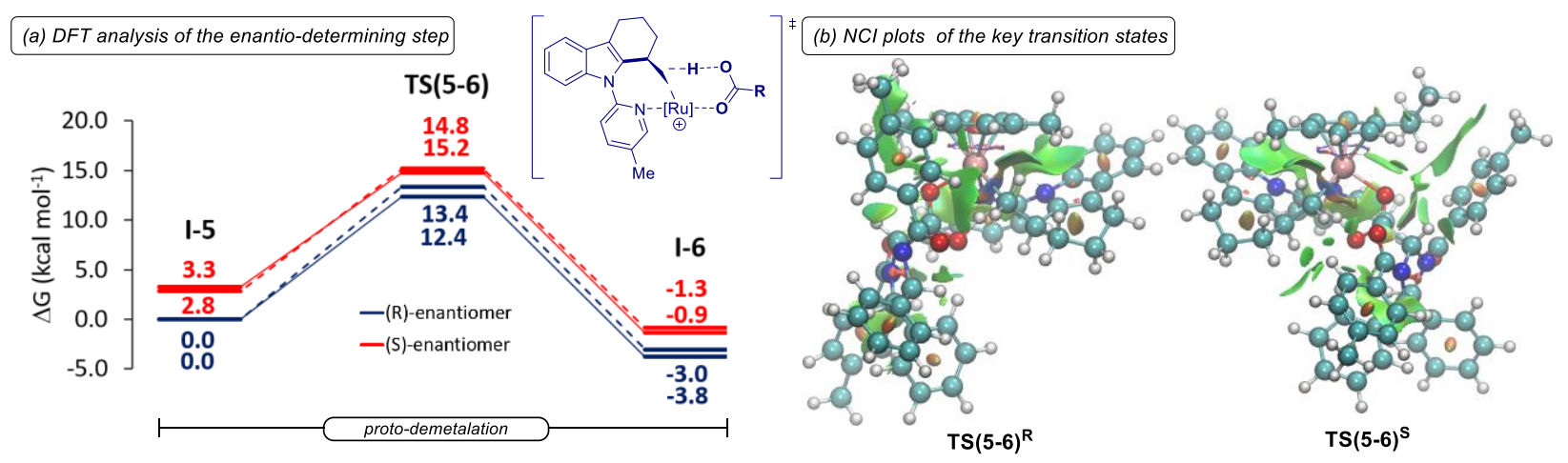

Figure S9. a) Computed relative Gibbs free energies $\left(\Delta \mathrm{G}_{298.15}\right)$ in $\mathrm{kcal} \mathrm{mol}^{-1}$ for the enatioinduction step with (full line) and without (dash line) dispersion corrections. b) Visualization of the noncovalent interactions calculated with the help of the NCIPLOT program, for the transition states involved in the proto-demetalation for both $(R)$ and $(S)$-enantiomers. In the plotted surfaces, red correspond to strong repulsive interactions, while green and blue correspond to weak and strong attractive interactions, respectively. Superscripts $R$ and $S$ correspond to the $(R)$ - and $(S)$ enantiomers respectively

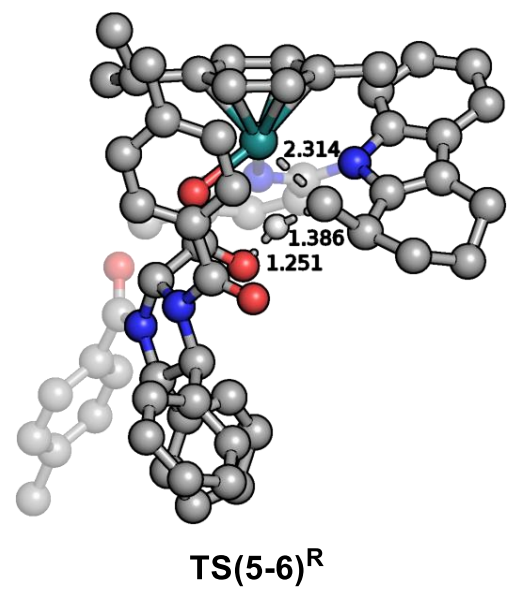

Figure S10. Computed transition state structure involved in the enantioinduction step at the PBE0$\mathrm{D} 3 \mathrm{BJ} / \mathrm{def} 2-\mathrm{SVP}$ level of theory for the major experimentally observed $(R)$-enantiomer. Nonrelevant hydrogens were omitted for clarity and bond distances are given in $\AA$. 
Table S11. Calculated electronic energies at the PW6B95-D4/def2-TZVP+SMD(Toluene) level of theory and Gibbs free energies with dispersion corrections for all structures in the present work (all in Hartree). ${ }^{a}$

\begin{tabular}{|c|c|c|}
\hline Structure & Electronic Energy & Total Gibbs Free Energy \\
\hline $\mathbf{I}-\mathbf{1}^{R}$ & -2981.046770 & -2980.064829 \\
\hline $\operatorname{TS}(1-2)^{R}$ & -2981.042414 & -2980.060095 \\
\hline $\mathrm{I}-2^{R}$ & -2981.055511 & -2980.071540 \\
\hline $\mathbf{I}-3^{R}$ & -1331.497962 & -1330.998985 \\
\hline $\operatorname{TS}(3-4)^{R}$ & -1331.479219 & -1330.980864 \\
\hline $\mathrm{I}-4^{R}$ & -1331.490903 & -1330.991559 \\
\hline $\mathbf{I}-5^{R}$ & -2981.065329 & -2980.073885 \\
\hline $\mathrm{TS}(5-6)^{R}$ & -2981.042839 & -2980.054166 \\
\hline $\mathrm{I}-\mathrm{6}^{\mathrm{R}}$ & -2981.072404 & -2980.079928 \\
\hline $\mathbf{I}-\mathbf{1}^{S}$ & -2981.047784 & -2980.063748 \\
\hline $\mathbf{T S}(1-2)^{S}$ & -2981.042414 & -2980.060047 \\
\hline $\mathbf{I}-2^{S}$ & -2981.056816 & -2980.070715 \\
\hline $\mathbf{I}-\mathbf{3}^{S}$ & -1331.497962 & -1330.998985 \\
\hline TS $(3-4)^{S}$ & -1331.479219 & -1330.980864 \\
\hline $\mathbf{I}-4^{S}$ & -1331.490902 & -1330.991557 \\
\hline $\mathbf{I}-\mathbf{5}^{S}$ & -2981.059907 & -2980.068703 \\
\hline $\mathbf{T S}(5-6)^{S}$ & -2981.038925 & -2980.050328 \\
\hline $\mathbf{I}-\mathbf{6}^{S}$ & -2981.068258 & -2980.076030 \\
\hline $\mathrm{RCO}_{2} \mathrm{H}$ & -1649.545413 & -1649.080180 \\
\hline
\end{tabular}

${ }^{a}$ Superscripts $R$ and $S$ correspond to the structures of the pathways that lead to the formation of the $(R)$ and $(S)$-enantiomers respectively. $\mathrm{RCO}_{2} \mathrm{H}$ corresponds to the structure of the chiral acid CA8. 
Table S12. Calculated electronic energies at the PBE0-D4/def2-TZVP+SMD(Toluene) level of theory and Gibbs free energies with dispersion corrections for all structures in the present work (all in Hartree). ${ }^{\mathrm{a}}$

\begin{tabular}{|c|c|c|}
\hline Structure & Electronic Energy & Total Gibbs Free Energy \\
\hline${\mathrm{I}-\mathbf{1}^{R}}^{R}$ & -2973.908377 & -2972.926436 \\
\hline $\operatorname{TS}(1-2)^{R}$ & -2973.906458 & -2972.924139 \\
\hline $\mathrm{I}-2^{R}$ & -2973.919194 & -2972.935223 \\
\hline $\mathrm{I}-3^{R}$ & -1328.366121 & -1327.867144 \\
\hline TS $(3-4)^{R}$ & -1328.349338 & -1327.850983 \\
\hline $\mathrm{I}-4^{R}$ & -1328.362175 & -1327.862831 \\
\hline $\mathrm{I}-5^{R}$ & -2973.928586 & -2972.937142 \\
\hline $\operatorname{TS}(5-6)^{R}$ & -2973.910528 & -2972.921855 \\
\hline $\mathbf{I}-6^{R}$ & -2973.935465 & -2972.942989 \\
\hline $\mathbf{I}^{-1}{ }^{S}$ & -2973.909463 & -2972.925427 \\
\hline TS $(1-2)^{S}$ & -2973.906312 & -2972.923945 \\
\hline $\mathbf{I}-\mathbf{2}^{S}$ & -2973.919036 & -2972.932935 \\
\hline $\mathbf{I}-\mathbf{3}^{S}$ & -1328.366121 & -1327.867144 \\
\hline $\operatorname{TS}(3-4)^{S}$ & -1328.349339 & -1327.850984 \\
\hline $\mathbf{I}-4^{S}$ & -1328.362175 & -1327.862830 \\
\hline $\mathbf{I}^{-5^{S}}$ & -2973.922768 & -2972.931564 \\
\hline $\operatorname{TS}(5-6)^{S}$ & -2973.906064 & -2972.917467 \\
\hline $\mathbf{I}-6^{S}$ & -2973.931627 & -2972.939399 \\
\hline $\mathrm{RCO}_{2} \mathrm{H}$ & -1645.537634 & -1645.072401 \\
\hline
\end{tabular}

${ }^{a}$ Superscripts $R$ and $S$ correspond to the structures of the pathways that lead to the formation of the $(R)$ and $(S)$-enantiomers respectively. $\mathrm{RCO}_{2} \mathrm{H}$ corresponds to the structure of the chiral acid CA8. 
Table S13. Calculated electronic energies at the PW6B95/def2-TZVP+SMD(Toluene) level of theory and Gibbs free energies with dispersion corrections for all structures in the present work (all in Hartree). ${ }^{\mathrm{a}}$

\begin{tabular}{|c|c|c|}
\hline Structure & Electronic Energy & Total Gibbs Free Energy \\
\hline${\mathrm{I}-\mathbf{1}^{R}}^{R}$ & -2980.854683 & -2979.872742 \\
\hline $\operatorname{TS}(1-2)^{R}$ & -2980.849766 & -2979.867447 \\
\hline $\mathrm{I}-2^{R}$ & -2980.863464 & -2979.879493 \\
\hline $\mathrm{I}-3^{R}$ & -1331.408552 & -1330.909575 \\
\hline $\operatorname{TS}(3-4)^{R}$ & -1331.390572 & -1330.892217 \\
\hline $\mathrm{I}-4^{R}$ & -1331.402539 & -1330.903195 \\
\hline $\mathrm{I}-5^{R}$ & -2980.872383 & -2979.880939 \\
\hline $\operatorname{TS}(5-6)^{R}$ & -2980.848313 & -2979.859640 \\
\hline $\mathbf{I}-6^{R}$ & -2980.878266 & -2979.885790 \\
\hline $\mathbf{I}^{-1}{ }^{S}$ & -2980.856779 & -2979.872743 \\
\hline TS $(1-2)^{S}$ & -2980.851122 & -2979.868755 \\
\hline $\mathbf{I}-\mathbf{2}^{S}$ & -2980.865717 & -2979.879616 \\
\hline $\mathbf{I}-\mathbf{3}^{S}$ & -1331.408552 & -1330.909575 \\
\hline $\operatorname{TS}(3-4)^{S}$ & -1331.390573 & -1330.892218 \\
\hline $\mathbf{I}-4^{S}$ & -1331.402538 & -1330.903193 \\
\hline $\mathbf{I}^{-5^{S}}$ & -2980.867642 & -2979.876438 \\
\hline $\operatorname{TS}(5-6)^{S}$ & -2980.845351 & -2979.856754 \\
\hline $\mathbf{I}-6^{S}$ & -2980.874578 & -2979.882350 \\
\hline $\mathrm{RCO}_{2} \mathrm{H}$ & -1649.457192 & -1648.991959 \\
\hline
\end{tabular}

a Superscripts $R$ and $S$ correspond to the structures of the pathways that lead to the formation of the $(R)$ and $(S)$-enantiomers respectively. $\mathrm{RCO}_{2} \mathrm{H}$ corresponds to the structure of the chiral acid CA8. 


\section{Cartesian coordinates of the optimized structures}

$\mathrm{I}-\mathbf{1}^{R}$

Lowest frequency $=8.4566 \mathrm{~cm}^{-1}$

Charge $=1$, Multiplicity $=1$

131

\begin{tabular}{rrr}
2.532084 & -1.783619 & 1.106168 \\
1.779482 & -1.796777 & -1.047179 \\
4.867927 & -2.321435 & 4.463965 \\
4.500820 & -3.483857 & 3.749330 \\
4.847649 & -4.456076 & 4.106919 \\
3.713454 & -3.431365 & 2.610667 \\
3.457475 & -4.342810 & 2.071202 \\
3.284172 & -2.166552 & 2.183384 \\
3.676319 & -0.978834 & 2.870540 \\
4.465959 & -1.072687 & 4.034287 \\
4.752664 & -0.171464 & 4.579543 \\
3.143898 & 0.135901 & 2.162843 \\
2.452801 & -0.367606 & 1.052157 \\
1.131339 & 1.737654 & 4.761877 \\
1.259882 & 2.725708 & 5.224284 \\
0.775492 & 1.732273 & 3.309697 \\
0.537111 & 0.705952 & 2.987183 \\
-0.137136 & 2.326457 & 3.138261 \\
1.884206 & 2.311546 & 2.426217 \\
2.051822 & 3.369534 & 2.687729 \\
1.554364 & 2.308853 & 1.373476 \\
3.217267 & 1.570021 & 2.559984 \\
3.984623 & 2.074817 & 1.946176 \\
3.570909 & 1.634646 & 3.599632 \\
1.313671 & 0.649215 & 5.512467 \\
1.192656 & -0.356033 & 5.094105 \\
1.570800 & 0.718473 & 6.572767 \\
1.915611 & -2.499385 & 0.097225 \\
1.402532 & -3.786859 & 0.214590 \\
1.476502 & -4.324874 & 1.158418 \\
0.740312 & -4.337375 & -0.878205 \\
0.321247 & -5.343653 & -0.798670 \\
0.569871 & -3.607702 & -2.057897 \\
1.107531 & -2.319613 & -2.075816 \\
0.972478 & -1.670969 & -2.941697 \\
-0.180005 & -4.121648 & -3.245485 \\
-0.665974 & -5.082099 & -3.028891 \\
0.489115 & -4.269202 & -4.107755 \\
-0.950934 & -3.390685 & -3.533216 \\
5.480099 & -2.419425 & 5.362323 \\
2.621704 & 0.116913 & -1.093091 \\
3.149559 & 1.892617 & -2.306843 \\
4.680409 & 0.703006 & -0.779578 \\
3.124667 & 0.722324 & -3.128121 \\
3.932266 & 1.868379 & -1.130764 \\
\hline .460007 & 0.686458 & -3.993078
\end{tabular}

\begin{tabular}{|c|c|c|c|}
\hline $\mathrm{H}$ & 3.906908 & 2.723375 & -0.453707 \\
\hline $\mathrm{H}$ & 3.743916 & -1.338386 & -3.339584 \\
\hline C & 2.276455 & 3.051809 & -2.641288 \\
\hline $\mathrm{H}$ & 1.260003 & 2.710339 & -2.878756 \\
\hline $\mathrm{H}$ & 2.204766 & 3.760720 & -1.808717 \\
\hline $\mathrm{H}$ & 2.681205 & 3.578945 & -3.519401 \\
\hline C & 5.502274 & -1.696627 & -1.281917 \\
\hline C & 6.132085 & -1.691272 & 0.102663 \\
\hline C & 6.568877 & -1.887532 & -2.363643 \\
\hline $\mathrm{H}$ & 4.800593 & -2.548277 & -1.341228 \\
\hline $\mathrm{H}$ & 5.392694 & -1.533281 & 0.898736 \\
\hline $\mathrm{H}$ & 6.623332 & -2.655170 & 0.296696 \\
\hline $\mathrm{H}$ & 6.904688 & -0.910856 & 0.187655 \\
\hline $\mathrm{H}$ & 6.133736 & -1.951252 & -3.372019 \\
\hline $\mathrm{H}$ & 7.283538 & -1.049778 & -2.357825 \\
\hline $\mathrm{H}$ & 7.133999 & -2.813787 & -2.184245 \\
\hline $\mathrm{H}$ & 1.377968 & -0.030622 & 0.873020 \\
\hline 0 & 0.680112 & 0.809449 & -1.285458 \\
\hline C & -0.304888 & 0.526037 & -0.532445 \\
\hline 0 & -0.244951 & -0.075634 & 0.543436 \\
\hline C & -1.678612 & 1.053917 & -1.000648 \\
\hline $\mathrm{N}$ & -2.659473 & -0.007480 & -1.000026 \\
\hline $\mathrm{N}$ & -2.232753 & 1.987313 & -0.035087 \\
\hline $\mathrm{H}$ & -1.557175 & 1.482434 & -2.004337 \\
\hline C & -3.639408 & 0.118316 & 0.081339 \\
\hline C & -2.488275 & -1.056552 & -1.844508 \\
\hline C & -3.042262 & 1.256632 & 0.936310 \\
\hline C & -1.546223 & 3.104279 & 0.416384 \\
\hline $\mathrm{H}$ & -4.601175 & 0.464328 & -0.329435 \\
\hline C & -3.873303 & -1.145629 & 0.871038 \\
\hline 0 & -1.465005 & -1.160363 & -2.512302 \\
\hline C & -3.606187 & -2.039401 & -1.919380 \\
\hline $\mathrm{H}$ & -2.393297 & 0.822852 & 1.714015 \\
\hline C & -4.117564 & 2.082004 & 1.588799 \\
\hline 0 & -1.530859 & 3.392421 & 1.596480 \\
\hline C & -0.897181 & 3.987324 & -0.595431 \\
\hline C & -2.797380 & -1.854119 & 1.418940 \\
\hline C & -5.172032 & -1.614304 & 1.068884 \\
\hline C & -3.383370 & -3.376184 & -1.580515 \\
\hline C & -4.879588 & -1.640290 & -2.327650 \\
\hline C & -4.443971 & 1.852079 & 2.925516 \\
\hline C & -4.850905 & 3.020012 & 0.856840 \\
\hline C & -1.346680 & 4.102165 & -1.913874 \\
\hline C & 0.110492 & 4.851287 & -0.148282 \\
\hline C & -3.025004 & -3.023597 & 2.137938 \\
\hline $\mathrm{H}$ & -1.780362 & -1.484083 & 1.265190 \\
\hline C & -5.398837 & -2.785651 & 1.789885 \\
\hline $\mathrm{H}$ & -6.013974 & -1.065078 & 0.640720 \\
\hline C & -4.431016 & -4.286675 & -1.611740 \\
\hline $\mathrm{H}$ & -2.390964 & -3.687234 & -1.248454 \\
\hline C & -5.917894 & -2.565615 & -2.384315 \\
\hline $\mathrm{H}$ & -5.061962 & -0.599443 & -2.60722 \\
\hline C & -5.490110 & 2.550087 & 3.525725 \\
\hline $\mathrm{H}$ & -3.868976 & 1.121931 & 3.500664 \\
\hline C & -5.890126 & 3.724760 & 1.457813 \\
\hline $\mathrm{H}$ & -4.594375 & 3.207737 & -0.189366 \\
\hline C & -0.778406 & 5.039675 & -2.77270 \\
\hline
\end{tabular}




$\begin{array}{lrrr}\text { H } & -2.176888 & 3.485339 & -2.263905 \\ \mathrm{C} & 0.674068 & 5.783290 & -1.008709 \\ \mathrm{H} & 0.418491 & 4.789759 & 0.897361 \\ \mathrm{C} & -4.325374 & -3.495658 & 2.322466 \\ \mathrm{H} & -2.179942 & -3.572022 & 2.562009 \\ \mathrm{H} & -6.420387 & -3.145178 & 1.933069 \\ \mathrm{C} & -5.718062 & -3.898447 & -2.009814 \\ \mathrm{H} & -4.253270 & -5.323299 & -1.313309 \\ \mathrm{H} & -6.908491 & -2.244034 & -2.716221 \\ \mathrm{C} & -6.214008 & 3.489286 & 2.794060 \\ \mathrm{H} & -5.736146 & 2.363888 & 4.573623 \\ \mathrm{H} & -6.450904 & 4.464081 & 0.881103 \\ \mathrm{C} & 0.242370 & 5.893552 & -2.339707 \\ \mathrm{H} & -1.150001 & 5.125112 & -3.797180 \\ \mathrm{H} & 1.456723 & 6.453549 & -0.642173 \\ \mathrm{H} & -4.502023 & -4.414902 & 2.885924 \\ \mathrm{H} & -7.029498 & 4.042203 & 3.265843 \\ \mathrm{C} & -6.849002 & -4.882767 & -2.002701 \\ \mathrm{H} & -7.710652 & -4.515798 & -2.576999 \\ \mathrm{H} & -6.542886 & -5.852157 & -2.423195 \\ \mathrm{H} & -7.191479 & -5.070625 & -0.971547 \\ \mathrm{C} & 0.836382 & 6.922656 & -3.253685 \\ \mathrm{H} & 0.476259 & 7.930655 & -2.990468 \\ \mathrm{H} & 1.933924 & 6.945068 & -3.175597 \\ \mathrm{H} & 0.567979 & 6.738779 & -4.302987\end{array}$

$\operatorname{TS}(1-2)^{R}$

Lowest frequency $=-126.6467 \mathrm{~cm}^{-1}$

Charge $=1$, Multiplicity $=1$

131

$\begin{array}{lrrr}\text { N } & 2.296624 & -1.956097 & 0.983678 \\ \mathrm{~N} & 1.791440 & -1.782657 & -1.231532 \\ \mathrm{C} & 3.670022 & -2.814628 & 4.782260 \\ \mathrm{C} & 3.362943 & -3.915968 & 3.957006 \\ \mathrm{H} & 3.506777 & -4.926921 & 4.344981 \\ \mathrm{C} & 2.886859 & -3.755827 & 2.664021 \\ \mathrm{H} & 2.682009 & -4.627259 & 2.043567 \\ \mathrm{C} & 2.717035 & -2.445843 & 2.196063 \\ \mathrm{C} & 3.052965 & -1.323066 & 3.005127 \\ \mathrm{C} & 3.523211 & -1.521414 & 4.316594 \\ \mathrm{H} & 3.763901 & -0.666254 & 4.950541 \\ \mathrm{C} & 2.837555 & -0.140084 & 2.233317 \\ \mathrm{C} & 2.367340 & -0.533176 & 0.974800 \\ \mathrm{C} & 0.831671 & 1.483889 & 4.903485 \\ \mathrm{H} & 1.042293 & 2.454467 & 5.373068 \\ \mathrm{C} & 0.538329 & 1.517466 & 3.437918 \\ \mathrm{H} & 0.280972 & 0.505811 & 3.083834 \\ \mathrm{H} & -0.346148 & 2.147929 & 3.247229 \\ \mathrm{C} & 1.692487 & 2.082519 & 2.609394 \\ \mathrm{H} & 1.909143 & 3.113843 & 2.934733 \\ \mathrm{H} & 1.385041 & 2.156685 & 1.553583 \\ \mathrm{C} & 2.984441 & 1.264875 & 2.713966 \\ \mathrm{H} & 3.777741 & 1.769970 & 2.137482 \\ \mathrm{H} & 3.323463 & 1.253813 & 3.760194 \\ \mathrm{C} & 0.853087 & 0.386261 & 5.662233 \\ \mathrm{H} & 0.644899 & -0.602052 & 5.238613\end{array}$

\begin{tabular}{|c|c|c|c|}
\hline $\mathrm{H}$ & 1.060804 & 0.433662 & 6.734561 \\
\hline C & 1.760404 & -2.565374 & -0.130173 \\
\hline C & 1.162049 & -3.822856 & -0.166455 \\
\hline $\mathrm{H}$ & 1.099860 & -4.430485 & 0.733801 \\
\hline C & 0.592269 & -4.253215 & -1.359173 \\
\hline $\mathrm{H}$ & 0.108911 & -5.232823 & -1.396858 \\
\hline C & 0.593536 & -3.437989 & -2.494444 \\
\hline C & 1.209673 & -2.194311 & -2.361687 \\
\hline $\mathrm{H}$ & 1.216106 & -1.483381 & -3.188740 \\
\hline C & -0.087681 & -3.807494 & -3.773143 \\
\hline $\mathrm{H}$ & -0.503267 & -4.822742 & -3.728982 \\
\hline $\mathrm{H}$ & 0.601934 & -3.757548 & -4.629311 \\
\hline $\mathrm{H}$ & -0.908476 & -3.096688 & -3.958823 \\
\hline $\mathrm{H}$ & 4.033736 & -2.991604 & 5.796128 \\
\hline $\mathrm{Ru}$ & 2.708788 & 0.082987 & -1.045849 \\
\hline C & 3.368743 & 1.854779 & -2.200032 \\
\hline C & 4.697506 & 0.699589 & -0.474283 \\
\hline C & 3.484854 & 0.695989 & -3.020423 \\
\hline C & 3.990774 & 1.850925 & -0.924168 \\
\hline C & 4.823902 & -0.468848 & -1.267814 \\
\hline $\mathrm{H}$ & 5.097249 & 0.694126 & 0.540090 \\
\hline C & 4.164836 & -0.444093 & -2.534526 \\
\hline $\mathrm{H}$ & 2.961788 & 0.657312 & -3.977302 \\
\hline $\mathrm{H}$ & 3.864976 & 2.708513 & -0.261977 \\
\hline $\mathrm{H}$ & 4.142327 & -1.362937 & -3.127851 \\
\hline C & 2.534945 & 3.007774 & -2.643584 \\
\hline $\mathrm{H}$ & 1.684456 & 2.670452 & -3.248744 \\
\hline $\mathrm{H}$ & 2.141937 & 3.572172 & -1.789697 \\
\hline $\mathrm{H}$ & 3.149632 & 3.688570 & -3.254306 \\
\hline C & 5.588971 & -1.703105 & -0.844374 \\
\hline C & 5.930218 & -1.738683 & 0.637659 \\
\hline C & 6.850594 & -1.833966 & -1.701450 \\
\hline $\mathrm{H}$ & 4.933536 & -2.564175 & -1.067009 \\
\hline $\mathrm{H}$ & 5.043010 & -1.616630 & 1.272844 \\
\hline $\mathrm{H}$ & 6.391262 & -2.702966 & 0.894204 \\
\hline $\mathrm{H}$ & 6.656767 & -0.954206 & 0.902346 \\
\hline $\mathrm{H}$ & 6.619951 & -1.866077 & -2.776798 \\
\hline $\mathrm{H}$ & 7.528937 & -0.983873 & -1.528337 \\
\hline $\mathrm{H}$ & 7.393805 & -2.755764 & -1.446744 \\
\hline $\mathrm{H}$ & 1.212038 & -0.122164 & 0.774396 \\
\hline 0 & 0.798058 & 0.867943 & -1.339255 \\
\hline C & -0.189708 & 0.646182 & -0.594227 \\
\hline 0 & -0.139025 & 0.059001 & 0.507791 \\
\hline C & -1.559619 & 1.191268 & -1.041714 \\
\hline $\mathrm{N}$ & -2.544273 & 0.134954 & -1.082204 \\
\hline $\mathrm{N}$ & -2.111457 & 2.094820 & -0.049479 \\
\hline $\mathrm{H}$ & -1.425703 & 1.649194 & -2.030806 \\
\hline C & -3.533126 & 0.229451 & -0.006190 \\
\hline C & -2.377248 & -0.881426 & -1.970064 \\
\hline C & -2.945906 & 1.343725 & 0.886475 \\
\hline C & -1.410429 & 3.182009 & 0.451417 \\
\hline $\mathrm{H}$ & -4.492029 & 0.585505 & -0.414390 \\
\hline C & -3.770409 & -1.059747 & 0.740973 \\
\hline 0 & -1.348247 & -0.966356 & -2.629579 \\
\hline C & -3.501085 & -1.850831 & -2.095161 \\
\hline $\mathrm{H}$ & -2.313452 & 0.887937 & 1.665301 \\
\hline C & -4.026765 & 2.159353 & 1.541719 \\
\hline 0 & -1.435031 & 3.446300 & 1.636302 \\
\hline C & -0.680506 & 4.054597 & -0.513222 \\
\hline C & -2.697566 & -1.793039 & 1.261468 \\
\hline C & -5.070759 & -1.529493 & 0.925229 \\
\hline
\end{tabular}




\begin{tabular}{|c|c|c|c|}
\hline C & -3.275540 & -3.207207 & -1.848477 \\
\hline C & -4.777550 & -1.425641 & -2.466533 \\
\hline C & -4.367138 & 1.907487 & 2.870945 \\
\hline C & -4.749826 & 3.111432 & 0.817902 \\
\hline C & -1.083838 & 4.239760 & -1.838563 \\
\hline C & 0.368484 & 4.835743 & -0.010975 \\
\hline C & -2.928354 & -2.985679 & 1.940117 \\
\hline $\mathrm{H}$ & -1.678462 & -1.424956 & 1.122184 \\
\hline C & -5.301558 & -2.723878 & 1.606109 \\
\hline $\mathrm{H}$ & -5.911072 & -0.961921 & 0.518336 \\
\hline C & -4.323581 & -4.114067 & -1.931842 \\
\hline $\mathrm{H}$ & -2.278264 & -3.538735 & -1.552439 \\
\hline C & -5.816192 & -2.345443 & -2.576157 \\
\hline $\mathrm{H}$ & -4.961702 & -0.369293 & -2.678502 \\
\hline C & -5.417903 & 2.597834 & 3.471908 \\
\hline $\mathrm{H}$ & -3.799041 & 1.167342 & 3.440260 \\
\hline C & -5.793665 & 3.808388 & 1.419927 \\
\hline $\mathrm{H}$ & -4.481537 & 3.316721 & -0.222083 \\
\hline C & -0.434809 & 5.167635 & -2.649080 \\
\hline $\mathrm{H}$ & -1.940703 & 3.688963 & -2.232006 \\
\hline C & 1.015045 & 5.755689 & -0.825007 \\
\hline $\mathrm{H}$ & 0.643967 & 4.720391 & 1.039165 \\
\hline C & -4.230599 & -3.457343 & 2.111226 \\
\hline $\mathrm{H}$ & -2.084603 & -3.551695 & 2.343193 \\
\hline $\mathrm{H}$ & -6.324508 & -3.083212 & 1.739099 \\
\hline C & -5.613733 & -3.700405 & -2.292482 \\
\hline $\mathrm{H}$ & -4.143311 & -5.168743 & -1.707357 \\
\hline $\mathrm{H}$ & -6.808884 & -2.003168 & -2.879830 \\
\hline C & -6.131964 & 3.550889 & 2.748549 \\
\hline $\mathrm{H}$ & -5.675006 & 2.394953 & 4.514005 \\
\hline $\mathrm{H}$ & -6.346618 & 4.558941 & 0.850265 \\
\hline C & 0.625712 & 5.939444 & -2.161020 \\
\hline $\mathrm{H}$ & -0.772063 & 5.310465 & -3.678978 \\
\hline $\mathrm{H}$ & 1.830602 & 6.359028 & -0.416682 \\
\hline $\mathrm{H}$ & -4.410608 & -4.394657 & 2.642843 \\
\hline $\mathrm{H}$ & -6.950993 & 4.097812 & 3.221137 \\
\hline C & 1.307746 & 6.958399 & -3.023164 \\
\hline $\mathrm{H}$ & 0.988751 & 7.976746 & -2.746807 \\
\hline $\mathrm{H}$ & 2.400836 & 6.921984 & -2.901605 \\
\hline $\mathrm{H}$ & 1.072335 & 6.815290 & -4.086632 \\
\hline C & -6.745131 & -4.682773 & -2.343329 \\
\hline $\mathrm{H}$ & -6.436382 & -5.630155 & -2.809295 \\
\hline $\mathrm{H}$ & -7.095795 & -4.921327 & -1.325475 \\
\hline $\mathrm{H}$ & -7.602255 & -4.286522 & -2.904811 \\
\hline
\end{tabular}

\section{I-2 ${ }^{R}$}

Lowest frequency $=9.6774 \mathrm{~cm}^{-1}$ Charge $=1$, Multiplicity $=1$

\section{1}

$\begin{array}{lrrr}\mathrm{N} & 2.042618 & -2.181628 & 1.038921 \\ \mathrm{~N} & 2.111605 & -1.818684 & -1.208162 \\ \mathrm{C} & 1.382043 & -3.095532 & 5.026358 \\ \mathrm{C} & 1.275775 & -4.163153 & 4.123779 \\ \mathrm{H} & 1.045769 & -5.163362 & 4.497066 \\ \mathrm{C} & 1.474139 & -3.982731 & 2.757602 \\ \mathrm{H} & 1.424665 & -4.844001 & 2.094221\end{array}$

\begin{tabular}{|c|c|c|c|}
\hline C & 1.774419 & -2.694803 & 2.307351 \\
\hline C & 1.896920 & -1.609422 & 3.210603 \\
\hline C & 1.699215 & -1.818962 & 4.580443 \\
\hline $\mathrm{H}$ & 1.786193 & -0.988284 & 5.282816 \\
\hline C & 2.263823 & -0.429275 & 2.454006 \\
\hline C & 2.369307 & -0.801671 & 1.128757 \\
\hline C & 0.000404 & 1.641777 & 4.912949 \\
\hline $\mathrm{H}$ & 0.369385 & 2.592668 & 5.321136 \\
\hline C & -0.016771 & 1.540963 & 3.420930 \\
\hline $\mathrm{H}$ & -0.326914 & 0.525134 & 3.122343 \\
\hline $\mathrm{H}$ & -0.781783 & 2.233011 & 3.028907 \\
\hline C & 1.312534 & 1.915507 & 2.766586 \\
\hline $\mathrm{H}$ & 1.611530 & 2.917087 & 3.120947 \\
\hline $\mathrm{H}$ & 1.176567 & 2.019297 & 1.676529 \\
\hline C & 2.461685 & 0.938358 & 3.035046 \\
\hline $\mathrm{H}$ & 3.390823 & 1.378623 & 2.641978 \\
\hline $\mathrm{H}$ & 2.607892 & 0.847042 & 4.122647 \\
\hline C & -0.423161 & 0.702129 & 5.760410 \\
\hline $\mathrm{H}$ & -0.806604 & -0.258465 & 5.400508 \\
\hline $\mathrm{H}$ & -0.416236 & 0.861736 & 6.841847 \\
\hline C & 1.804629 & -2.684745 & -0.217505 \\
\hline C & 1.254485 & -3.933843 & -0.520247 \\
\hline $\mathrm{H}$ & 0.998548 & -4.632314 & 0.270267 \\
\hline C & 1.007675 & -4.251783 & -1.846429 \\
\hline $\mathrm{H}$ & 0.570087 & -5.224137 & -2.087589 \\
\hline C & 1.282449 & -3.340198 & -2.872440 \\
\hline C & 1.846549 & -2.132972 & -2.482429 \\
\hline $\mathrm{H}$ & 2.075343 & -1.359554 & -3.217470 \\
\hline C & 0.903699 & -3.591290 & -4.296758 \\
\hline $\mathrm{H}$ & 0.936884 & -4.661573 & -4.543239 \\
\hline $\mathrm{H}$ & 1.561850 & -3.056083 & -4.995346 \\
\hline $\mathrm{H}$ & -0.124572 & -3.232726 & -4.470759 \\
\hline $\mathrm{H}$ & 1.224014 & -3.274295 & 6.091909 \\
\hline Ru & 2.965868 & -0.014300 & -0.646479 \\
\hline C & 3.804704 & 1.790988 & -1.715167 \\
\hline C & 4.757675 & 0.735500 & 0.306737 \\
\hline C & 4.156588 & 0.645382 & -2.457167 \\
\hline C & 4.111918 & 1.828081 & -0.316243 \\
\hline C & 5.079455 & -0.454289 & -0.410578 \\
\hline $\mathrm{H}$ & 4.938487 & 0.769360 & 1.381202 \\
\hline C & 4.721473 & -0.473909 & -1.789245 \\
\hline $\mathrm{H}$ & 3.902342 & 0.584421 & -3.516467 \\
\hline $\mathrm{H}$ & 3.809696 & 2.692457 & 0.277227 \\
\hline $\mathrm{H}$ & 4.869990 & -1.402604 & -2.347883 \\
\hline C & 3.052473 & 2.915689 & -2.341975 \\
\hline $\mathrm{H}$ & 2.738159 & 2.671705 & -3.364173 \\
\hline $\mathrm{H}$ & 2.157035 & 3.156056 & -1.753806 \\
\hline $\mathrm{H}$ & 3.682328 & 3.817938 & -2.373015 \\
\hline C & 5.786401 & -1.644817 & 0.197707 \\
\hline C & 5.664830 & -1.739886 & 1.712200 \\
\hline C & 7.253333 & -1.629343 & -0.240147 \\
\hline $\mathrm{H}$ & 5.308389 & -2.539457 & -0.239070 \\
\hline $\mathrm{H}$ & 4.618315 & -1.725981 & 2.045362 \\
\hline $\mathrm{H}$ & 6.121125 & -2.676032 & 2.064095 \\
\hline $\mathrm{H}$ & 6.198716 & -0.917267 & 2.214060 \\
\hline $\mathrm{H}$ & 7.356415 & -1.619683 & -1.335642 \\
\hline $\mathrm{H}$ & 7.768625 & -0.739173 & 0.153858 \\
\hline $\mathrm{H}$ & 7.778095 & -2.517596 & 0.141222 \\
\hline $\mathrm{H}$ & 0.639439 & -0.280890 & 0.948963 \\
\hline 0 & 1.049855 & 0.849135 & -1.042811 \\
\hline C & -0.055223 & 0.587558 & -0.572528 \\
\hline
\end{tabular}




\begin{tabular}{|c|c|c|}
\hline-0.246875 & -0.085438 & 0.518838 \\
\hline-1.337278 & 1.164835 & -1.180876 \\
\hline-2.362185 & 0.149666 & -1.266386 \\
\hline-1.935731 & 2.158636 & -0.314634 \\
\hline-1.059935 & 1.553735 & -2.170224 \\
\hline-3.486403 & 0.407852 & -0.366051 \\
\hline-2.028107 & -0.973192 & -1.966700 \\
\hline-2.924718 & 1.519288 & 0.549985 \\
\hline-1.261049 & 3.274341 & 0.167870 \\
\hline-4.328104 & 0.837263 & -0.933040 \\
\hline-3.982100 & -0.770352 & 0.431119 \\
\hline-0.865903 & -1.100414 & -2.344712 \\
\hline-3.063424 & -1.991672 & -2.253854 \\
\hline-2.428659 & 1.049886 & 1.415351 \\
\hline-4.007408 & 2.438543 & 1.042145 \\
\hline-1.403745 & 3.630389 & 1.318996 \\
\hline-0.420429 & 4.058522 & -0.779570 \\
\hline-3.097304 & -1.679658 & 1.019745 \\
\hline-5.352889 & -0.913792 & 0.649243 \\
\hline-2.675634 & -3.335111 & -2.256453 \\
\hline-4.383688 & -1.663083 & -2.573146 \\
\hline-4.481120 & 2.305521 & 2.347508 \\
\hline-4.602033 & 3.371350 & 0.187806 \\
\hline-0.682857 & 4.146092 & -2.150133 \\
\hline 0.586558 & 4.863085 & -0.229979 \\
\hline-3.581682 & -2.724553 & 1.800640 \\
\hline-2.022375 & -1.569543 & 0.865522 \\
\hline-5.838078 & -1.956713 & 1.436436 \\
\hline-6.048135 & -0.200276 & 0.199146 \\
\hline-3.601981 & -4.331216 & -2.533568 \\
\hline-1.638402 & -3.580883 & -2.021985 \\
\hline-5.300057 & -2.664020 & -2.871146 \\
\hline-4.697084 & -0.618089 & -2.612542 \\
\hline-5.538060 & 3.094990 & 2.796163 \\
\hline-4.013256 & 1.580817 & 3.018735 \\
\hline-5.652076 & 4.166591 & 0.637794 \\
\hline-4.229178 & 3.485050 & -0.834028 \\
\hline 0.062959 & 5.002983 & -2.954912 \\
\hline-1.505288 & 3.577274 & -2.589207 \\
\hline 1.328071 & 5.713182 & -1.038385 \\
\hline 0.753758 & 4.821169 & 0.848326 \\
\hline-4.953726 & -2.867215 & 2.010065 \\
\hline-2.881116 & -3.430879 & 2.252622 \\
\hline-6.913302 & -2.058225 & 1.599509 \\
\hline-4.932728 & -4.015375 & -2.840463 \\
\hline-3.292357 & -5.379485 & -2.514043 \\
\hline-6.326341 & -2.392936 & -3.131262 \\
\hline-6.124351 & 4.027697 & 1.94314 \\
\hline-5.900291 & 2.985330 & 3.820758 \\
\hline-6.104494 & 4.901041 & -0.032525 \\
\hline 1.080775 & 5.799638 & -2.416962 \\
\hline-0.162922 & 5.070221 & -4.022235 \\
\hline 2.109804 & 6.335196 & -0.593884 \\
\hline-5.332897 & -3.687601 & 2.623637 \\
\hline-6.948345 & 4.651763 & 2.29637 \\
\hline 1.862133 & 6.745887 & -3.277378 \\
\hline 1.487228 & 7.775846 & -3.158514 \\
\hline 2.926594 & 6.759054 & -2.999788 \\
\hline 1.783895 & 6.487671 & -4.342374 \\
\hline-5.941287 & -5.092063 & -3.10285 \\
\hline-6.704322 & -4.764859 & -3.82328 \\
\hline
\end{tabular}

$\begin{array}{llll}H & -5.468518 & -6.005948 & -3.489041 \\ H & -6.466395 & -5.361898 & -2.171295\end{array}$

I-3 ${ }^{R}$

Lowest frequency $=22.3806 \mathrm{~cm}^{-1}$

Charge $=1$, Multiplicity $=1$

65

\begin{tabular}{|c|c|c|c|}
\hline $\mathrm{N}$ & 1.545409 & 0.660823 & -0.164056 \\
\hline $\mathrm{N}$ & -0.503749 & 1.474491 & -0.714128 \\
\hline C & 5.468058 & -0.623982 & 0.236097 \\
\hline C & 5.279004 & 0.762912 & 0.196486 \\
\hline $\mathrm{H}$ & 6.140869 & 1.428336 & 0.276352 \\
\hline C & 4.006489 & 1.316373 & 0.064851 \\
\hline $\mathrm{H}$ & 3.895483 & 2.399371 & 0.064992 \\
\hline C & 2.925132 & 0.442958 & -0.035218 \\
\hline C & 3.097287 & -0.963944 & 0.028331 \\
\hline C & 4.385195 & -1.493200 & 0.158219 \\
\hline $\mathrm{H}$ & 4.538403 & -2.573751 & 0.203357 \\
\hline C & 1.788620 & -1.582739 & -0.048932 \\
\hline C & 0.871907 & -0.576692 & -0.130194 \\
\hline C & -0.458422 & -1.281873 & -2.152703 \\
\hline $\mathrm{H}$ & 0.411395 & -0.707941 & -2.490175 \\
\hline C & -0.259027 & -2.777856 & -2.175394 \\
\hline $\mathrm{H}$ & -1.081275 & -3.272862 & -1.635405 \\
\hline $\mathrm{H}$ & -0.380255 & -3.079369 & -3.232502 \\
\hline C & 1.092751 & -3.320420 & -1.707113 \\
\hline $\mathrm{H}$ & 1.884611 & -2.923364 & -2.364417 \\
\hline $\mathrm{H}$ & 1.082424 & -4.410183 & -1.870154 \\
\hline C & 1.489380 & -3.032984 & -0.254696 \\
\hline $\mathrm{H}$ & 0.694525 & -3.378783 & 0.427170 \\
\hline $\mathrm{H}$ & 2.374804 & -3.635431 & -0.003132 \\
\hline C & -1.700024 & -0.654400 & -2.286939 \\
\hline $\mathrm{H}$ & -2.611158 & -1.252935 & -2.365293 \\
\hline $\mathrm{H}$ & -1.753636 & 0.331411 & -2.756607 \\
\hline C & 0.810864 & 1.747184 & -0.541516 \\
\hline C & 1.299199 & 3.041656 & -0.776459 \\
\hline $\mathrm{H}$ & 2.356947 & 3.257164 & -0.658060 \\
\hline C & 0.420297 & 4.027895 & -1.178516 \\
\hline $\mathrm{H}$ & 0.799238 & 5.035906 & -1.365925 \\
\hline C & -0.947019 & 3.751971 & -1.358498 \\
\hline C & -1.344078 & 2.447476 & -1.112181 \\
\hline $\mathrm{H}$ & -2.385980 & 2.146111 & -1.241312 \\
\hline C & -1.919235 & 4.801283 & -1.800101 \\
\hline $\mathrm{H}$ & -1.632086 & 5.220261 & -2.776814 \\
\hline $\mathrm{H}$ & -1.952265 & 5.637849 & -1.085102 \\
\hline $\mathrm{H}$ & -2.936354 & 4. 397018 & -1.894009 \\
\hline $\mathrm{H}$ & 6.478388 & -1.025298 & 0.338736 \\
\hline Ru & -1.134958 & -0.415616 & -0.195754 \\
\hline $\mathrm{C}$ & -2.977816 & -1.696501 & 0.307390 \\
\hline C & -1.053759 & -1.378732 & 1.799780 \\
\hline C & -3.325279 & -0.327722 & 0.514281 \\
\hline C & -1.846798 & -2.215489 & 0.982089 \\
\hline C & -1.365023 & -0.006435 & 1.995642 \\
\hline $\mathrm{H}$ & -0.147273 & -1.788625 & 2.246445 \\
\hline C & -2.525513 & 0.486810 & 1.329328 \\
\hline $\mathrm{H}$ & -4.196173 & 0.091438 & 0.006884 \\
\hline
\end{tabular}




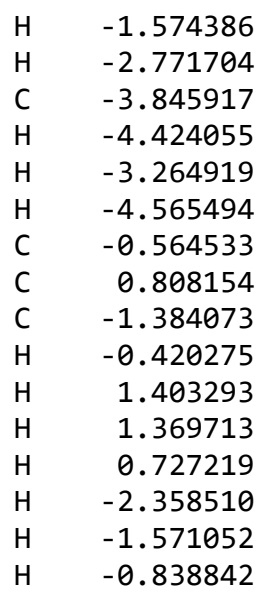

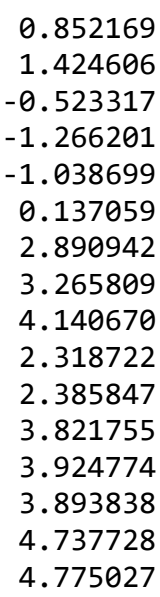

\section{TS(3-4) ${ }^{R}$}

Lowest frequency $=-280.3058 \mathrm{~cm}^{-1}$ Charge $=1$, Multiplicity $=1$

65

\begin{tabular}{|c|c|c|c|}
\hline $\mathrm{N}$ & 1.445369 & 0.747554 & -0.446402 \\
\hline $\mathrm{N}$ & -0.682977 & 1.555417 & -0.535086 \\
\hline C & 5.354974 & -0.208242 & 0.539185 \\
\hline C & 5.043200 & 1.159517 & 0.491349 \\
\hline $\mathrm{H}$ & 5.818226 & 1.895083 & 0.716359 \\
\hline C & 3.764984 & 1.605612 & 0.168009 \\
\hline $\mathrm{H}$ & 3.554683 & 2.673539 & 0.166120 \\
\hline C & 2.795334 & 0.643114 & -0.119700 \\
\hline C & 3.084275 & -0.743291 & -0.034937 \\
\hline C & 4.382905 & -1.164749 & 0.285347 \\
\hline $\mathrm{H}$ & 4.620943 & -2.228800 & 0.347573 \\
\hline C & 1.870064 & -1.467143 & -0.288465 \\
\hline C & 0.865980 & -0.544412 & -0.518081 \\
\hline C & -0.003736 & -0.963305 & -2.229635 \\
\hline $\mathrm{H}$ & 0.738342 & -0.297773 & -2.690049 \\
\hline C & 0.218179 & -2.433696 & -2.519998 \\
\hline $\mathrm{H}$ & -0.633568 & -2.999394 & -2.109649 \\
\hline $\mathrm{H}$ & 0.132836 & -2.513435 & -3.618268 \\
\hline C & 1.523319 & -3.074896 & -2.080907 \\
\hline $\mathrm{H}$ & 2.369165 & -2.617312 & -2.620434 \\
\hline $\mathrm{H}$ & 1.508856 & -4.138462 & -2.365467 \\
\hline C & 1.759763 & -2.922704 & -0.576709 \\
\hline $\mathrm{H}$ & 0.935297 & -3.393147 & -0.017106 \\
\hline $\mathrm{H}$ & 2.678583 & -3.446226 & -0.277175 \\
\hline C & -1.364567 & -0.492359 & -2.287396 \\
\hline $\mathrm{H}$ & -2.118682 & -1.233559 & -2.569794 \\
\hline $\mathrm{H}$ & -1.534981 & 0.493822 & -2.730478 \\
\hline C & 0.632539 & 1.841473 & -0.600330 \\
\hline C & 1.067193 & 3.148388 & -0.858312 \\
\hline $\mathrm{H}$ & 2.126789 & 3.361525 & -0.974152 \\
\hline C & 0.124204 & 4.148026 & -1.010943 \\
\hline $\mathrm{H}$ & 0.455362 & 5.169393 & -1.215661 \\
\hline C & -1.248907 & 3.860977 & -0.936237 \\
\hline C & -1.587127 & 2.533314 & -0.715546 \\
\hline $\mathrm{H}$ & -2.632463 & 2.218986 & -0.687969 \\
\hline C & -2.293175 & 4.919823 & -1.106283 \\
\hline
\end{tabular}

$\begin{array}{rrrr}\text { H } & -2.173215 & 5.443753 & -2.066790 \\ \text { H } & -2.219628 & 5.678002 & -0.311151 \\ \text { H } & -3.306594 & 4.497244 & -1.076235 \\ \text { H } & 6.370604 & -0.518680 & 0.792189 \\ \text { Ru } & -1.186502 & -0.421986 & -0.187805 \\ \text { C } & -2.944183 & -1.795940 & 0.077582 \\ \text { C } & -0.981978 & -1.724332 & 1.583051 \\ \text { C } & -3.293336 & -0.491793 & 0.527771 \\ \text { C } & -1.778130 & -2.398225 & 0.614471 \\ \text { C } & -1.324199 & -0.443925 & 2.071014 \\ \text { H } & -0.057495 & -2.196005 & 1.919174 \\ \text { C } & -2.467137 & 0.163249 & 1.472748 \\ \text { H } & -4.168530 & 0.014117 & 0.116876 \\ \text { H } & -1.472929 & -3.383152 & 0.257949 \\ \text { H } & -2.699996 & 1.198068 & 1.740758 \\ \text { C } & -3.789193 & -2.514502 & -0.923882 \\ \text { H } & -4.294374 & -1.816222 & -1.604526 \\ \text { H } & -3.199707 & -3.225608 & -1.518444 \\ \text { H } & -4.566998 & -3.088766 & -0.395664 \\ \text { C } & -0.534572 & 0.298198 & 3.123026 \\ \text { C } & 0.929842 & -0.113218 & 3.193732 \\ \text { C } & -1.227503 & 0.150456 & 4.479838 \\ \text { H } & -0.572408 & 1.364387 & 2.836897 \\ \text { H } & 1.424015 & -0.047502 & 2.214142 \\ \text { H } & 1.470988 & 0.542212 & 3.890858 \\ \text { H } & 1.046945 & -1.142129 & 3.568601 \\ \text { H } & -2.273097 & 0.491647 & 4.445347 \\ \text { H } & -1.227766 & -0.901811 & 4.804786 \\ \text { H } & -0.703916 & 0.740284 & 5.246670\end{array}$

\section{I- $4^{R}$}

Lowest frequency $=21.6420 \mathrm{~cm}^{-1}$

Charge $=1$, Multiplicity $=1$

65

$\begin{array}{lrrr}\text { N } & 1.117654 & 0.878115 & -1.156587 \\ \mathrm{~N} & -1.104694 & 1.256035 & -0.710188 \\ \mathrm{C} & 4.694575 & 1.107805 & 0.903229 \\ \mathrm{C} & 4.021753 & 2.322778 & 0.652433 \\ \mathrm{H} & 4.464363 & 3.256672 & 1.006007 \\ \mathrm{C} & 2.816341 & 2.367404 & -0.031623 \\ \mathrm{H} & 2.309296 & 3.316638 & -0.206610 \\ \mathrm{C} & 2.289783 & 1.150778 & -0.481322 \\ \mathrm{C} & 2.931096 & -0.083392 & -0.203564 \\ \mathrm{C} & 4.159311 & -0.095806 & 0.484093 \\ \mathrm{H} & 4.669831 & -1.038671 & 0.691697 \\ \mathrm{C} & 2.099178 & -1.119276 & -0.717157 \\ \mathrm{C} & 0.947814 & -0.533176 & -1.250036 \\ \mathrm{C} & 0.090526 & -1.205980 & -2.348568 \\ \mathrm{H} & 0.312762 & -0.656421 & -3.283012 \\ \mathrm{C} & 0.517893 & -2.674233 & -2.510281 \\ \mathrm{H} & -0.041189 & -3.274895 & -1.772699 \\ \mathrm{H} & 0.176663 & -3.010375 & -3.501003 \\ \mathrm{C} & 2.000078 & -2.951208 & -2.330763 \\ \mathrm{H} & 2.589826 & -2.388189 & -3.074013 \\ \mathrm{H} & 2.207862 & -4.017382 & -2.506100 \\ \mathrm{C} & 2.451072 & -2.550740 & -0.925198\end{array}$




\begin{tabular}{rrrr} 
H & 1.958982 & -3.193384 & -0.174948 \\
$\mathrm{H}$ & 3.533070 & -2.695002 & -0.791705 \\
$\mathrm{C}$ & -1.380374 & -1.118017 & -1.961938 \\
$\mathrm{H}$ & -1.913332 & -2.065809 & -2.117440 \\
$\mathrm{H}$ & -1.927672 & -0.317347 & -2.478439 \\
$\mathrm{C}$ & 0.028862 & 1.743451 & -1.236017 \\
$\mathrm{C}$ & 0.090995 & 3.012055 & -1.806892 \\
$\mathrm{H}$ & 1.020255 & 3.358111 & -2.260096 \\
$\mathrm{C}$ & -1.056393 & 3.792225 & -1.809109 \\
$\mathrm{H}$ & -1.031831 & 4.791079 & -2.251990 \\
$\mathrm{C}$ & -2.253610 & 3.291824 & -1.277662 \\
$\mathrm{C}$ & -2.218715 & 2.000343 & -0.756868 \\
$\mathrm{H}$ & -3.122800 & 1.532727 & -0.362065 \\
$\mathrm{C}$ & -3.517239 & 4.093952 & -1.282458 \\
$\mathrm{H}$ & -3.786164 & 4.398922 & -2.305196 \\
$\mathrm{H}$ & -3.400953 & 5.013747 & -0.688693 \\
$\mathrm{H}$ & -4.359743 & 3.526072 & -0.865738 \\
$\mathrm{H}$ & 5.644802 & 1.125289 & 1.440100 \\
$\mathrm{Ru}$ & -0.969939 & -0.691496 & 0.026445 \\
$\mathrm{C}$ & -2.049657 & -2.469793 & 0.760530 \\
$\mathrm{C}$ & -0.091654 & -1.470845 & 1.923664 \\
$\mathrm{C}$ & -2.772690 & -1.271077 & 1.045481 \\
$\mathrm{C}$ & -0.699321 & -2.530196 & 1.174963 \\
$\mathrm{C}$ & -0.807819 & -0.319724 & 2.288970 \\
$\mathrm{H}$ & 0.965878 & -1.553555 & 2.178796 \\
$\mathrm{C}$ & -2.132649 & -0.220710 & 1.758735 \\
$\mathrm{H}$ & -3.800134 & -1.152606 & 0.696933 \\
$\mathrm{H}$ & -0.100314 & -3.400225 & 0.900479 \\
$\mathrm{H}$ & -2.679797 & 0.714916 & 1.908562 \\
$\mathrm{C}$ & -2.691785 & -3.598997 & 0.023349 \\
$\mathrm{H}$ & -3.440595 & -3.238198 & -0.694587 \\
$\mathrm{H}$ & -1.951195 & -4.204714 & -0.515830 \\
$\mathrm{H}$ & -3.205732 & -4.257690 & 0.741485 \\
$\mathrm{C}$ & -0.240288 & 0.808584 & 3.117120 \\
$\mathrm{C}$ & 1.280532 & 0.828970 & 3.160942 \\
$\mathrm{C}$ & -0.839629 & 0.760539 & 4.524966 \\
$\mathrm{H}$ & -0.578682 & 1.744461 & 2.636913 \\
$\mathrm{H}$ & 1.720032 & 0.836213 & 2.153228 \\
$\mathrm{H}$ & 1.631683 & 1.728350 & 3.686495 \\
\hline & -1.681710 & -0.039134 & 3.707213 \\
$\mathrm{H}$ & -0.490409 & -1.615026 & 5.123352
\end{tabular}

\section{I-5 $5^{R}$}

Lowest frequency $=10.2863 \mathrm{~cm}^{-1}$ Charge $=1$, Multiplicity $=1$

131

$\begin{array}{lllr}\text { N } & 3.810648 & -1.851368 & 0.898493 \\ \mathrm{~N} & 2.085651 & -1.474972 & -0.673924 \\ \mathrm{C} & 7.649295 & -3.045233 & 1.893047 \\ \mathrm{C} & 7.217073 & -3.344417 & 0.590587 \\ \mathrm{H} & 7.896092 & -3.850898 & -0.098597 \\ \mathrm{C} & 5.939062 & -3.002431 & 0.158279 \\ \mathrm{H} & 5.613513 & -3.234045 & -0.858255 \\ \mathrm{C} & 5.102055 & -2.354019 & 1.068005\end{array}$

\begin{tabular}{|c|c|c|c|}
\hline C & 5.522617 & -2.037281 & 2.382225 \\
\hline C & 6.814952 & -2.391616 & 2.791609 \\
\hline $\mathrm{H}$ & 7.161761 & -2.151394 & 3.799143 \\
\hline C & 4.430702 & -1.346590 & 3.019573 \\
\hline C & 3.429761 & -1.223890 & 2.097797 \\
\hline C & 2.132978 & -0.516364 & 2.287274 \\
\hline $\mathrm{H}$ & 1.325870 & -1.249420 & 2.066381 \\
\hline C & 1.979565 & -0.085810 & 3.766726 \\
\hline $\mathrm{H}$ & 2.326766 & 0.958288 & 3.846822 \\
\hline $\mathrm{H}$ & 0.909366 & -0.064118 & 4.023310 \\
\hline C & 2.764252 & -0.923379 & 4.769192 \\
\hline $\mathrm{H}$ & 2.402235 & -1.965778 & 4.760992 \\
\hline $\mathrm{H}$ & 2.590852 & -0.534844 & 5.784420 \\
\hline C & 4.256496 & -0.915787 & 4.438717 \\
\hline $\mathrm{H}$ & 4.679800 & 0.090971 & 4.609846 \\
\hline $\mathrm{H}$ & 4.808533 & -1.595821 & 5.106576 \\
\hline C & 2.034487 & 0.725922 & 1.383783 \\
\hline $\mathrm{H}$ & 2.775677 & 1.436806 & 1.780935 \\
\hline $\mathrm{H}$ & 1.060270 & 1.218395 & 1.564343 \\
\hline C & 2.914037 & -2.335108 & -0.054246 \\
\hline C & 2.875595 & -3.704076 & -0.345835 \\
\hline $\mathrm{H}$ & 3.538873 & -4.375765 & 0.199616 \\
\hline C & 1.991152 & -4.179472 & -1.297906 \\
\hline $\mathrm{H}$ & 1.959644 & -5.248174 & -1.525769 \\
\hline C & 1.118969 & -3.293955 & -1.944874 \\
\hline C & 1.203145 & -1.957366 & -1.573512 \\
\hline $\mathrm{H}$ & 0.526710 & -1.231538 & -2.026804 \\
\hline C & 0.151085 & -3.732178 & -2.999023 \\
\hline $\mathrm{H}$ & -0.302891 & -4.700407 & -2.741541 \\
\hline $\mathrm{H}$ & 0.663765 & -3.864440 & -3.965793 \\
\hline $\mathrm{H}$ & -0.646771 & -2.988584 & -3.131021 \\
\hline $\mathrm{H}$ & 8.659125 & -3.326613 & 2.198748 \\
\hline $\mathrm{Ru}$ & 2.372222 & 0.626144 & -0.688133 \\
\hline 0 & 0.271669 & 0.890706 & -0.929610 \\
\hline C & -0.709589 & 0.426711 & -0.364006 \\
\hline 0 & -0.651362 & -0.465432 & 0.588756 \\
\hline C & -2.095279 & 1.002614 & -0.629871 \\
\hline $\mathrm{H}$ & 0.288857 & -0.654818 & 0.783656 \\
\hline $\mathrm{N}$ & -3.107582 & -0.020702 & -0.685506 \\
\hline $\mathrm{N}$ & -2.521217 & 1.841965 & 0.474502 \\
\hline $\mathrm{H}$ & -2.011714 & 1.544270 & -1.583486 \\
\hline C & -4.110701 & 0.121622 & 0.370357 \\
\hline C & -2.890022 & -1.032558 & -1.573219 \\
\hline C & -3.410639 & 1.086604 & 1.351977 \\
\hline C & -1.669811 & 2.769161 & 1.081982 \\
\hline $\mathrm{H}$ & -5.003385 & 0.631824 & -0.026009 \\
\hline C & -4.532159 & -1.154294 & 1.049284 \\
\hline 0 & -1.794915 & -1.097328 & -2.131187 \\
\hline C & -3.980298 & -1.997700 & -1.838548 \\
\hline $\mathrm{H}$ & -2.820460 & 0.499947 & 2.076462 \\
\hline C & -4.399899 & 1.937710 & 2.099748 \\
\hline 0 & -1.613058 & 2.846557 & 2.289354 \\
\hline C & -0.882304 & 3.675805 & 0.199619 \\
\hline C & -3.590440 & -2.113007 & 1.436499 \\
\hline C & -5.877274 & -1.352757 & 1.360787 \\
\hline C & -3.666106 & -3.353491 & -1.969865 \\
\hline C & -5.304418 & -1.584494 & -2.010474 \\
\hline C & -4.777567 & 1.577678 & 3.393804 \\
\hline C & -5.010411 & 3.035704 & 1.487373 \\
\hline C & -1.286516 & 4.039241 & -1.087557 \\
\hline C & 0.261350 & 4.273425 & 0.744818 \\
\hline
\end{tabular}




\begin{tabular}{|c|c|c|c|c|c|c|c|}
\hline C & -3.995747 & -3.261434 & 2.109328 & $\mathrm{H}$ & 1.042410 & 6.633024 & -3.115222 \\
\hline $\mathrm{H}$ & -2.533959 & -1.960038 & 1.203032 & & & & \\
\hline $\mathrm{C}$ & -6.282747 & -2.499287 & 2.041847 & & & & \\
\hline $\mathrm{H}$ & -6.615410 & -0.602163 & 1.066755 & \multirow{2}{*}{\multicolumn{4}{|c|}{$\mathrm{TS}(5-6)^{R}$}} \\
\hline C & -4.666337 & -4.280782 & -2.228245 & & & & \\
\hline $\mathrm{H}$ & -2.630459 & -3.674806 & -1.848216 & \multicolumn{4}{|c|}{ Lowest frequency $=-1332.8073 \mathrm{~cm}^{-1}$} \\
\hline C & -6.295962 & -2.515416 & -2.294218 & \multirow{2}{*}{\multicolumn{4}{|c|}{ Charge $=1$, Multiplicity $=1$}} \\
\hline $\mathrm{H}$ & -5.562139 & -0.525282 & -1.944463 & & & & \\
\hline C & -5.754234 & 2.305825 & 4.069986 & \multirow{2}{*}{\multicolumn{2}{|c|}{131}} & & \\
\hline $\mathrm{H}$ & -4.299383 & 0.721303 & 3.875783 & & & & \\
\hline C & -5.980091 & 3.769120 & 2.165719 & & & & \\
\hline $\mathrm{H}$ & -4.712789 & 3.325317 & 0.475963 & $\mathrm{~N}$ & 3.504004 & -2.012993 & 0.682619 \\
\hline C & -0.543847 & 4.957259 & -1.826270 & $\mathrm{~N}$ & 1.918203 & -1.310804 & -0.947057 \\
\hline $\mathrm{H}$ & -2.210908 & 3.636387 & -1.506435 & C & 7.317158 & -3.513307 & 1.269763 \\
\hline C & 0.994308 & 5.192011 & 0.006727 & $\mathrm{C}$ & 6.828756 & -3.536051 & -0.047435 \\
\hline $\mathrm{H}$ & 0.545955 & 4.012746 & 1.766312 & $\mathrm{H}$ & 7.460891 & -3.925084 & -0.848492 \\
\hline C & -5.343473 & -3.458310 & 2.412872 & C & 5.552662 & -3.071103 & -0.351458 \\
\hline $\mathrm{H}$ & -3.255347 & -4.009700 & 2.401624 & $\mathrm{H}$ & 5.175539 & -3.098022 & -1.376501 \\
\hline $\mathrm{H}$ & -7.338797 & -2.643897 & 2.280120 & $\mathrm{C}$ & 4.778165 & -2.580676 & 0.701594 \\
\hline C & -5.999939 & -3.880854 & -2.390708 & C & 5.248977 & -2.556554 & 2.035678 \\
\hline $\mathrm{H}$ & -4.411905 & -5.340749 & -2.308638 & C & 6.539533 & -3.027863 & 2.313959 \\
\hline $\mathrm{H}$ & -7.324889 & -2.177538 & -2.440929 & $\mathrm{H}$ & 6.927008 & -3.011861 & 3.335137 \\
\hline C & -6.356004 & 3.403754 & 3.458284 & C & 4.199951 & -1.985133 & 2.839982 \\
\hline $\mathrm{H}$ & -6.041273 & 2.017798 & 5.083751 & $\mathrm{C}$ & 3.172232 & -1.651168 & 2.000711 \\
\hline $\mathrm{H}$ & -6.444693 & 4.632677 & 1.684222 & $\mathrm{C}$ & 1.912039 & -0.951726 & 2.384700 \\
\hline C & 0.609998 & 5.547116 & -1.296739 & $\mathrm{H}$ & 1.049480 & -1.597348 & 2.131067 \\
\hline $\mathrm{H}$ & -0.878059 & 5.238775 & -2.828080 & C & 1.911089 & -0.696330 & 3.907340 \\
\hline $\mathrm{H}$ & 1.880602 & 5.656371 & 0.448405 & $\mathrm{H}$ & 2.397262 & 0.278089 & 4.091293 \\
\hline $\mathrm{H}$ & -5.660097 & -4.360230 & 2.941527 & $\mathrm{H}$ & 0.869709 & -0.583106 & 4.245035 \\
\hline $\mathrm{H}$ & -7.116368 & 3.979458 & 3.990807 & C & 2.641640 & -1.751593 & 4.727174 \\
\hline C & 4.420934 & 1.336572 & -0.472015 & $\mathrm{H}$ & 2.173786 & -2.739102 & 4.573527 \\
\hline C & 3.501666 & 0.542388 & -2.659573 & $\mathrm{H}$ & 2.547527 & -1.518774 & 5.798690 \\
\hline C & 3.519643 & 2.448351 & -0.559993 & C & 4.112077 & -1.825677 & 4.321941 \\
\hline C & 4.342796 & 0.375072 & -1.510455 & $\mathrm{H}$ & 4.645455 & -0.916794 & 4.656897 \\
\hline C & 2.611670 & 1.618677 & -2.728244 & $\mathrm{H}$ & 4.613867 & -2.671293 & 4.818978 \\
\hline $\mathrm{H}$ & 3.483681 & -0.237389 & -3.421579 & C & 1.771670 & 0.394318 & 1.653212 \\
\hline C & 2.614906 & 2.550639 & -1.630239 & $\mathrm{H}$ & 2.672148 & 0.975073 & 1.868407 \\
\hline $\mathrm{H}$ & 3.467052 & 3.174714 & 0.253875 & $\mathrm{H}$ & 0.968299 & 0.984178 & 2.131559 \\
\hline $\mathrm{H}$ & 4.957468 & -0.524591 & -1.426807 & C & 2.585609 & -2.305719 & -0.336290 \\
\hline $\mathrm{H}$ & 1.857867 & 3.336536 & -1.603781 & $\mathrm{C}$ & 2.399748 & -3.634444 & -0.722513 \\
\hline C & 5.403127 & 1.210443 & 0.645546 & $\mathrm{H}$ & 2.939624 & -4.414118 & -0.184011 \\
\hline $\mathrm{H}$ & 4.975955 & 1.544984 & 1.600391 & C & 1.547184 & -3.933698 & -1.772609 \\
\hline $\mathrm{H}$ & 5.749307 & 0.176002 & 0.764690 & $\mathrm{H}$ & 1.405224 & -4.972299 & -2.082308 \\
\hline $\mathrm{H}$ & 6.281445 & 1.840377 & 0.431801 & $\mathrm{C}$ & 0.858039 & -2.905219 & -2.423076 \\
\hline C & 1.592955 & 1.816477 & -3.824211 & C & 1.065690 & -1.613062 & -1.942974 \\
\hline C & 1.387640 & 0.595678 & -4.707682 & $\mathrm{H}$ & 0.508720 & -0.780539 & -2.373642 \\
\hline C & 1.956993 & 3.054492 & -4.647526 & $\mathrm{C}$ & -0.044886 & -3.150129 & -3.590870 \\
\hline $\mathrm{H}$ & 0.641716 & 2.024774 & -3.303356 & $\mathrm{H}$ & -0.476568 & -4.160133 & -3.552790 \\
\hline $\mathrm{H}$ & 1.116492 & -0.293418 & -4.119367 & $\mathrm{H}$ & 0.519718 & -3.073316 & -4.534753 \\
\hline $\mathrm{H}$ & 0.573144 & 0.779630 & -5.422766 & $\mathrm{H}$ & -0.857811 & -2.411366 & -3.615464 \\
\hline $\mathrm{H}$ & 2.289205 & 0.361219 & -5.295577 & $\mathrm{H}$ & 8.324016 & -3.884188 & 1.472339 \\
\hline $\mathrm{H}$ & 2.051451 & 3.951121 & -4.016513 & $\mathrm{Ru}$ & 2.299752 & 0.730417 & -0.574926 \\
\hline $\mathrm{H}$ & 2.912861 & 2.909889 & -5.174672 & 0 & 0.312996 & 1.162132 & -1.061764 \\
\hline $\mathrm{H}$ & 1.181470 & 3.254991 & -5.401557 & C & -0.625765 & 0.632622 & -0.422477 \\
\hline C & -7.082062 & -4.888378 & -2.633462 & 0 & -0.421445 & -0.212090 & 0.488652 \\
\hline $\mathrm{H}$ & -6.704417 & -5.765847 & -3.177083 & C & -2.042302 & 1.167294 & -0.635988 \\
\hline $\mathrm{H}$ & -7.493014 & -5.248386 & -1.675379 & $\mathrm{H}$ & 0.775380 & 0.029310 & 0.761445 \\
\hline $\mathrm{H}$ & -7.915795 & -4.457697 & -3.205503 & $\mathrm{~N}$ & -3.010990 & 0.110803 & -0.766697 \\
\hline C & 1.408558 & 6.542008 & -2.083701 & $\mathrm{~N}$ & -2.485298 & 1.875611 & 0.555087 \\
\hline $\mathrm{H}$ & 1.357740 & 7.539902 & -1.619604 & $\mathrm{H}$ & -2.016488 & 1.794484 & -1.538107 \\
\hline $\mathrm{H}$ & 2.472798 & 6.261035 & -2.120004 & C & -3.954189 & 0.042816 & 0.349223 \\
\hline
\end{tabular}




\begin{tabular}{|c|c|c|c|}
\hline C & -2.842382 & -0.768110 & -1.789465 \\
\hline C & -3.311599 & 0.994554 & 1.379502 \\
\hline C & -1.657665 & 2.755642 & 1.249665 \\
\hline $\mathrm{H}$ & -4.925700 & 0.463082 & 0.045113 \\
\hline C & -4.167401 & -1.344909 & 0.899972 \\
\hline 0 & -1.818199 & -0.718780 & -2.464347 \\
\hline C & -3.940228 & -1.737323 & -2.040175 \\
\hline $\mathrm{H}$ & -2.665895 & 0.413857 & 2.058601 \\
\hline C & -4.350406 & 1.717895 & 2.193486 \\
\hline 0 & -1.607229 & 2.723062 & 2.461032 \\
\hline C & -0.870762 & 3.757229 & 0.471972 \\
\hline C & -3.074547 & -2.148569 & 1.244908 \\
\hline C & -5.460424 & -1.832145 & 1.086868 \\
\hline C & -3.667991 & -3.107409 & -2.061327 \\
\hline C & -5.244160 & -1.293544 & -2.269719 \\
\hline C & -4.707049 & 1.221615 & 3.447923 \\
\hline C & -5.023656 & 2.829068 & 1.679094 \\
\hline C & -1.234730 & 4.222727 & -0.794240 \\
\hline C & 0.234123 & 4.334030 & 1.111109 \\
\hline C & -3.279210 & -3.426804 & 1.754688 \\
\hline $\mathrm{H}$ & -2.059488 & -1.770083 & 1.098321 \\
\hline C & -5.665253 & -3.112180 & 1.600101 \\
\hline $\mathrm{H}$ & -6.315869 & -1.208340 & 0.816646 \\
\hline C & -4.696164 & -4.017321 & -2.268357 \\
\hline $\mathrm{H}$ & -2.649524 & -3.456495 & -1.880539 \\
\hline C & -6.262776 & -2.210850 & -2.505894 \\
\hline $\mathrm{H}$ & -5.464664 & -0.223089 & -2.274938 \\
\hline C & -5.724701 & 1.828069 & 4.181445 \\
\hline $\mathrm{H}$ & -4.179180 & 0.354834 & 3.853633 \\
\hline C & -6.034368 & 3.441327 & 2.415493 \\
\hline $\mathrm{H}$ & -4.742653 & 3.223827 & 0.699108 \\
\hline C & -0.488257 & 5.217095 & -1.421396 \\
\hline $\mathrm{H}$ & -2.129552 & 3.836547 & -1.285367 \\
\hline C & 0.970947 & 5.330752 & 0.485140 \\
\hline $\mathrm{H}$ & 0.481360 & 3.995570 & 2.119601 \\
\hline C & -4.575359 & -3.913557 & 1.931046 \\
\hline $\mathrm{H}$ & -2.421261 & -4.050366 & 2.017673 \\
\hline $\mathrm{H}$ & -6.682728 & -3.484746 & 1.738209 \\
\hline C & -6.012090 & -3.587522 & -2.490244 \\
\hline $\mathrm{H}$ & -4.477931 & -5.088378 & -2.255612 \\
\hline $\mathrm{H}$ & -7.276948 & -1.851817 & -2.698814 \\
\hline C & -6.388991 & 2.940239 & 3.667887 \\
\hline $\mathrm{H}$ & -5.994918 & 1.433744 & 5.163634 \\
\hline $\mathrm{H}$ & -6.548399 & 4.316303 & 2.010824 \\
\hline C & 0.627671 & 5.788040 & -0.797476 \\
\hline $\mathrm{H}$ & -0.791655 & 5.575132 & -2.408597 \\
\hline $\mathrm{H}$ & 1.825437 & 5.777352 & 1.001666 \\
\hline $\mathrm{H}$ & -4.734806 & -4.918111 & 2.329611 \\
\hline $\mathrm{H}$ & -7.181648 & 3.420725 & 4.245853 \\
\hline C & 4.372951 & 1.308538 & 0.003716 \\
\hline C & 3.620692 & 0.869227 & -2.318280 \\
\hline C & 3.503116 & 2.451235 & 0.010233 \\
\hline C & 4.387635 & 0.517057 & -1.163045 \\
\hline C & 2.790590 & 2.005557 & -2.317542 \\
\hline $\mathrm{H}$ & 3.618405 & 0.197384 & -3.177091 \\
\hline C & 2.734647 & 2.785312 & -1.113323 \\
\hline $\mathrm{H}$ & 3.385832 & 3.037813 & 0.924637 \\
\hline $\mathrm{H}$ & 4.974407 & -0.404168 & -1.164214 \\
\hline $\mathrm{H}$ & 2.013931 & 3.601700 & -1.039971 \\
\hline C & 5.233876 & 0.982891 & 1.179014 \\
\hline $\mathrm{H}$ & 4.727742 & 1.189853 & 2.132031 \\
\hline
\end{tabular}

$\begin{array}{rrrr}\text { H } & 5.550211 & -0.067573 & 1.173205 \\ \mathrm{H} & 6.137096 & 1.613109 & 1.142989 \\ \mathrm{C} & 1.895122 & 2.399597 & -3.466098 \\ \mathrm{C} & 1.730887 & 1.321297 & -4.525410 \\ \mathrm{C} & 2.402372 & 3.711780 & -4.070310 \\ \mathrm{H} & 0.907134 & 2.589526 & -3.013240 \\ \mathrm{H} & 1.365140 & 0.377587 & -4.095654 \\ \mathrm{H} & 0.998385 & 1.644402 & -5.278524 \\ \mathrm{H} & 2.675038 & 1.120480 & -5.056308 \\ \mathrm{H} & 2.465583 & 4.512124 & -3.317925 \\ \mathrm{H} & 3.401497 & 3.583925 & -4.515174 \\ \mathrm{H} & 1.720549 & 4.051711 & -4.863590 \\ \mathrm{C} & 1.412391 & 6.881776 & -1.457246 \\ \mathrm{H} & 1.151248 & 7.861226 & -1.023968 \\ \mathrm{H} & 2.494849 & 6.747010 & -1.312533 \\ \mathrm{H} & 1.210028 & 6.934309 & -2.535833 \\ \mathrm{C} & -7.119563 & -4.580246 & -2.677025 \\ \mathrm{H} & -6.800498 & -5.428176 & -3.300574 \\ \mathrm{H} & -7.435076 & -4.993268 & -1.704479 \\ \mathrm{H} & -8.001952 & -4.121516 & -3.143870\end{array}$

\section{I-6 ${ }^{R}$}

Lowest frequency $=11.4789 \mathrm{~cm}^{-1}$

Charge $=1$, Multiplicity $=1$

\section{1}

$\begin{array}{lrrr}\text { N } & 3.576748 & -1.924058 & 0.775232 \\ \text { N } & 1.895134 & -1.479357 & -0.849959 \\ \mathrm{C} & 7.482728 & -3.285695 & 0.891644 \\ \mathrm{C} & 6.796536 & -3.443296 & -0.324692 \\ \mathrm{H} & 7.308789 & -3.898270 & -1.175154 \\ \mathrm{C} & 5.473809 & -3.033693 & -0.464628 \\ \mathrm{H} & 4.938246 & -3.171490 & -1.407071 \\ \mathrm{C} & 4.859228 & -2.455828 & 0.647864 \\ \mathrm{C} & 5.525094 & -2.307901 & 1.886790 \\ \mathrm{C} & 6.859127 & -2.724732 & 1.999377 \\ \mathrm{H} & 7.397649 & -2.612408 & 2.943080 \\ \mathrm{C} & 4.585889 & -1.703835 & 2.795948 \\ \mathrm{C} & 3.427250 & -1.476278 & 2.103243 \\ \mathrm{C} & 2.208766 & -0.793011 & 2.635330 \\ \mathrm{H} & 1.342624 & -1.476377 & 2.568688 \\ \mathrm{C} & 2.439883 & -0.390581 & 4.106115 \\ \mathrm{H} & 2.908059 & 0.610095 & 4.122284 \\ \mathrm{H} & 1.462404 & -0.275780 & 4.597976 \\ \mathrm{C} & 3.335383 & -1.344981 & 4.884047 \\ \mathrm{H} & 2.888565 & -2.353717 & 4.894353 \\ \mathrm{H} & 3.402960 & -1.016974 & 5.932152 \\ \mathrm{C} & 4.724272 & -1.417656 & 4.254889 \\ \mathrm{H} & 5.269472 & -0.470606 & 4.423568 \\ \mathrm{H} & 5.329956 & -2.203980 & 4.733069 \\ \mathrm{C} & 1.865064 & 0.464923 & 1.842566 \\ \mathrm{H} & 2.717582 & 1.149984 & 1.800549 \\ \mathrm{H} & 1.007820 & 1.001526 & 2.276194 \\ \mathrm{C} & 2.542528 & -2.364643 & -0.075058 \\ \mathrm{C} & 2.241532 & -3.723485 & -0.138244 \\ \mathrm{H} & 2.780110 & -4.407909 & 0.518526 \\ \mathrm{C} & 1.283076 & -4.172559 & -1.034396\end{array}$




\begin{tabular}{|c|c|c|c|}
\hline $\mathrm{H}$ & 1.046431 & -5.238028 & -1.093069 \\
\hline C & 0.613699 & -3.258077 & -1.852880 \\
\hline C & 0.943551 & -1.912983 & -1.692380 \\
\hline $\mathrm{H}$ & 0.395471 & -1.148208 & -2.245214 \\
\hline C & -0.405324 & -3.672564 & -2.866073 \\
\hline $\mathrm{H}$ & -0.942725 & -4.573876 & -2.538998 \\
\hline $\mathrm{H}$ & 0.083591 & -3.916443 & -3.823698 \\
\hline $\mathrm{H}$ & -1.128840 & -2.864604 & -3.045470 \\
\hline $\mathrm{H}$ & 8.520767 & -3.616343 & 0.965323 \\
\hline $\mathrm{Ru}$ & 2.321838 & 0.580691 & -0.781719 \\
\hline 0 & 0.329079 & 0.894626 & -1.034215 \\
\hline C & -0.637058 & 0.355210 & -0.369198 \\
\hline 0 & -0.543882 & -0.541007 & 0.452948 \\
\hline C & -1.978360 & 1.070610 & -0.612872 \\
\hline $\mathrm{H}$ & 1.427662 & 0.136053 & 0.857220 \\
\hline $\mathrm{N}$ & -3.070698 & 0.140926 & -0.716421 \\
\hline $\mathrm{N}$ & -2.327523 & 1.855483 & 0.565395 \\
\hline $\mathrm{H}$ & -1.887410 & 1.674849 & -1.525312 \\
\hline C & -3.968495 & 0.177629 & 0.439115 \\
\hline C & -3.024690 & -0.777887 & -1.716689 \\
\hline C & -3.175358 & 1.047091 & 1.438765 \\
\hline C & -1.400646 & 2.679450 & 1.191495 \\
\hline $\mathrm{H}$ & -4.896930 & 0.709706 & 0.177063 \\
\hline C & -4.319796 & -1.175712 & 1.004174 \\
\hline 0 & -2.019801 & -0.879753 & -2.415250 \\
\hline C & -4.250745 & -1.595550 & -1.924227 \\
\hline $\mathrm{H}$ & -2.546301 & 0.393504 & 2.065051 \\
\hline C & -4.087019 & 1.852393 & 2.323990 \\
\hline 0 & -1.246967 & 2.644553 & 2.396081 \\
\hline C & -0.637961 & 3.652154 & 0.351555 \\
\hline C & -3.312219 & -2.094088 & 1.322548 \\
\hline C & -5.653505 & -1.516471 & 1.227103 \\
\hline C & -4.179603 & -2.990459 & -1.916847 \\
\hline C & -5.483708 & -0.975523 & -2.137127 \\
\hline C & -4.416719 & 1.368247 & 3.590662 \\
\hline C & -4.676547 & 3.034789 & 1.868612 \\
\hline C & -1.069498 & 4.106162 & -0.897861 \\
\hline C & 0.510737 & 4.221630 & 0.913954 \\
\hline C & -3.644974 & -3.340931 & 1.843103 \\
\hline $\mathrm{H}$ & -2.267206 & -1.825465 & 1.142378 \\
\hline C & -5.985191 & -2.765541 & 1.750063 \\
\hline $\mathrm{H}$ & -6.441550 & -0.802261 & 0.976318 \\
\hline C & -5.332137 & -3.747667 & -2.078485 \\
\hline $\mathrm{H}$ & -3.218902 & -3.479230 & -1.747315 \\
\hline C & -6.629488 & -1.741410 & -2.327409 \\
\hline $\mathrm{H}$ & -5.548070 & 0.115286 & -2.161588 \\
\hline C & -5.323560 & 2.056475 & 4.394074 \\
\hline $\mathrm{H}$ & -3.956170 & 0.444153 & 3.949109 \\
\hline C & -5.575787 & 3.728212 & 2.674385 \\
\hline $\mathrm{H}$ & -4.417653 & 3.418784 & 0.878368 \\
\hline C & -0.347996 & 5.080278 & -1.583053 \\
\hline $\mathrm{H}$ & -1.996727 & 3.723917 & -1.327812 \\
\hline C & 1.223753 & 5.199427 & 0.230963 \\
\hline $\mathrm{H}$ & 0.809556 & 3.896451 & 1.912898 \\
\hline C & -4.981750 & -3.681871 & 2.055504 \\
\hline $\mathrm{H}$ & -2.854577 & -4.055405 & 2.086392 \\
\hline $\mathrm{H}$ & -7.033660 & -3.023495 & 1.915721 \\
\hline C & -6.578388 & -3.138795 & -2.282178 \\
\hline $\mathrm{H}$ & -5.268902 & -4.838418 & -2.042179 \\
\hline $\mathrm{H}$ & -7.586549 & -1.244454 & -2.506043 \\
\hline C & -5.903357 & 3.239058 & 3.938781 \\
\hline
\end{tabular}

$\begin{array}{rrrr}\mathrm{H} & -5.573726 & 1.669622 & 5.384567 \\ \mathrm{H} & -6.024164 & 4.657173 & 2.314281 \\ \mathrm{C} & 0.811160 & 5.644853 & -1.034774 \\ \mathrm{H} & -0.705006 & 5.428575 & -2.555867 \\ \mathrm{H} & 2.111208 & 5.644050 & 0.690802 \\ \mathrm{H} & -5.240606 & -4.662380 & 2.462000 \\ \mathrm{H} & -6.609075 & 3.782817 & 4.570918 \\ \mathrm{C} & 4.420512 & 1.268024 & -0.428952 \\ \mathrm{C} & 3.464173 & 0.531249 & -2.579081 \\ \mathrm{C} & 3.513582 & 2.382067 & -0.473417 \\ \mathrm{C} & 4.381474 & 0.353285 & -1.497552 \\ \mathrm{C} & 2.592521 & 1.649237 & -2.637304 \\ \mathrm{H} & 3.383451 & -0.249088 & -3.336680 \\ \mathrm{C} & 2.643764 & 2.583714 & -1.551805 \\ \mathrm{H} & 3.444910 & 3.054223 & 0.385384 \\ \mathrm{H} & 4.999769 & -0.545594 & -1.450695 \\ \mathrm{H} & 1.906423 & 3.386641 & -1.497423 \\ \mathrm{C} & 5.362712 & 1.094361 & 0.715698 \\ \mathrm{H} & 4.914882 & 1.409584 & 1.667680 \\ \mathrm{H} & 5.698647 & 0.054711 & 0.813791 \\ \mathrm{H} & 6.248969 & 1.726285 & 0.542710 \\ \mathrm{C} & 1.556037 & 1.854132 & -3.713842 \\ \mathrm{C} & 1.372809 & 0.661915 & -4.639577 \\ \mathrm{C} & 1.891611 & 3.127042 & -4.496072 \\ \mathrm{H} & 0.609360 & 2.016845 & -3.170537 \\ \mathrm{H} & 1.122050 & -0.255146 & -4.088001 \\ \mathrm{H} & 0.544538 & 0.855565 & -5.335314 \\ \mathrm{H} & 2.272066 & 0.471519 & -5.246957 \\ \mathrm{H} & 1.957000 & 4.008259 & -3.840524 \\ \mathrm{H} & 2.849528 & 3.026347 & -5.029778 \\ \mathrm{H} & 1.109192 & 3.324519 & -5.243077 \\ \mathrm{C} & 1.562533 & 6.723685 & -1.755341 \\ \mathrm{H} & 1.232589 & 7.718408 & -1.412738 \\ \mathrm{H} & 2.644238 & 6.661169 & -1.567169 \\ \mathrm{H} & 1.394430 & 6.681641 & -2.840802 \\ \mathrm{C} & -7.820395 & -3.967121 & -2.419574 \\ \mathrm{H} & -7.649280 & -4.847983 & -3.055823 \\ \mathrm{H} & -8.148549 & -4.337846 & -1.434299 \\ \mathrm{H} & -8.649438 & -3.388072 & -2.849012\end{array}$

\section{I-1 ${ }^{s}$}

Lowest frequency $=11.9510 \mathrm{~cm}^{-1}$

Charge $=1$, Multiplicity $=1$

131

$\begin{array}{rrrr}\text { N } & 2.654908 & -1.496170 & 1.517381 \\ \text { N } & 2.447279 & 0.761670 & 1.286532 \\ \text { C } & 4.348364 & -5.211566 & 2.089817 \\ \text { C } & 4.521433 & -4.265723 & 3.125665 \\ \text { H } & 5.088042 & -4.552299 & 4.014581 \\ \text { C } & 3.994204 & -2.987147 & 3.050751 \\ \text { H } & 4.154049 & -2.271997 & 3.857399 \\ \text { C } & 3.269543 & -2.658220 & 1.895837 \\ \text { C } & 3.116146 & -3.586028 & 0.824244 \\ \text { C } & 3.654629 & -4.883507 & 0.942559 \\ \text { H } & 3.523811 & -5.610098 & 0.138565 \\ \text { C } & 2.394659 & -2.939154 & -0.219186\end{array}$




\begin{tabular}{|c|c|c|c|}
\hline C & 2.135246 & -1.626447 & 0.202716 \\
\hline C & -0.357994 & -5.283478 & -0.321132 \\
\hline $\mathrm{H}$ & -0.638020 & -5.956639 & -1.142880 \\
\hline C & -0.493656 & -3.819422 & -0.594086 \\
\hline $\mathrm{H}$ & -0.283542 & -3.248408 & 0.324158 \\
\hline $\mathrm{H}$ & -1.539063 & -3.590184 & -0.859533 \\
\hline C & 0.404507 & -3.325307 & -1.730136 \\
\hline $\mathrm{H}$ & 0.132618 & -3.834777 & -2.669607 \\
\hline $\mathrm{H}$ & 0.205181 & -2.255949 & -1.902815 \\
\hline C & 1.895080 & -3.571444 & -1.473283 \\
\hline $\mathrm{H}$ & 2.479533 & -3.197425 & -2.331657 \\
\hline $\mathrm{H}$ & 2.088097 & -4.653280 & -1.423759 \\
\hline C & 0.080785 & -5.814227 & 0.822213 \\
\hline $\mathrm{H}$ & 0.372361 & -5.181172 & 1.667292 \\
\hline $\mathrm{H}$ & 0.154527 & -6.895781 & 0.963187 \\
\hline C & 2.524276 & -0.272625 & 2.145497 \\
\hline C & 2.394171 & -0.080967 & 3.518550 \\
\hline $\mathrm{H}$ & 2.420353 & -0.932615 & 4.197021 \\
\hline C & 2.156536 & 1.204884 & 3.986453 \\
\hline $\mathrm{H}$ & 2.031973 & 1.370969 & 5.059282 \\
\hline C & 2.031887 & 2.281943 & 3.100661 \\
\hline C & 2.189210 & 1.990434 & 1.746312 \\
\hline $\mathrm{H}$ & 2.092637 & 2.766067 & 0.985344 \\
\hline C & 1.689995 & 3.666952 & 3.550400 \\
\hline $\mathrm{H}$ & 0.658423 & 3.907754 & 3.244974 \\
\hline $\mathrm{H}$ & 1.765475 & 3.763238 & 4.641272 \\
\hline $\mathrm{H}$ & 2.356871 & 4.414628 & 3.095517 \\
\hline $\mathrm{H}$ & 4.775057 & -6.209776 & 2.203562 \\
\hline $\mathrm{Ru}$ & 2.610164 & 0.317612 & -0.754111 \\
\hline $\mathrm{C}$ & 2.675894 & 1.004628 & -2.842345 \\
\hline C & 4.245514 & -0.614239 & -1.829776 \\
\hline C & 3.223349 & 2.009071 & -1.980907 \\
\hline C & 3.199049 & -0.304970 & -2.754648 \\
\hline C & 4.806178 & 0.362699 & -0.977937 \\
\hline H & 4.568674 & -1.651306 & -1.734754 \\
\hline C & 4.236788 & 1.676002 & -1.055955 \\
\hline $\mathrm{H}$ & 2.763441 & 2.999756 & -1.955623 \\
\hline $\mathrm{H}$ & 2.743664 & -1.101329 & -3.345205 \\
\hline H & 4.560602 & 2.432556 & -0.335265 \\
\hline C & 1.500549 & 1.309756 & -3.711997 \\
\hline H & 0.993140 & 2.223018 & -3.376444 \\
\hline $\mathrm{H}$ & 0.770749 & 0.488000 & -3.694208 \\
\hline H & 1.840177 & 1.456491 & -4.749668 \\
\hline C & 5.926768 & 0.091116 & -0.000274 \\
\hline C & 6.191480 & -1.387650 & 0.237276 \\
\hline C & 7.193647 & 0.807697 & -0.475065 \\
\hline $\mathrm{H}$ & 5.617344 & 0.544876 & 0.958503 \\
\hline $\mathrm{H}$ & 5.287155 & -1.926875 & 0.549635 \\
\hline $\mathrm{H}$ & 6.942548 & -1.513620 & 1.029868 \\
\hline $\mathrm{H}$ & 6.590613 & -1.875476 & -0.665873 \\
\hline $\mathrm{H}$ & 7.035735 & 1.889050 & -0.602620 \\
\hline H & 7.532124 & 0.400999 & -1.440792 \\
\hline $\mathrm{H}$ & 8.007034 & 0.670896 & 0.252462 \\
\hline $\mathrm{H}$ & 1.070499 & -1.223555 & 0.116191 \\
\hline 0 & 0.675821 & 0.987429 & -0.862698 \\
\hline C & -0.383967 & 0.432005 & -0.426954 \\
\hline 0 & -0.442108 & -0.553274 & 0.307287 \\
\hline C & -1.658032 & 1.187447 & -0.853796 \\
\hline$N$ & -2.801145 & 0.319983 & -0.901278 \\
\hline $\mathrm{N}$ & -2.020166 & 2.162150 & 0.157139 \\
\hline $\mathrm{H}$ & -1.451450 & 1.634008 & -1.832294 \\
\hline
\end{tabular}

\begin{tabular}{|c|c|c|c|}
\hline C & -3.691587 & 0.533913 & 0.238317 \\
\hline C & -2.744308 & -0.648641 & -1.865376 \\
\hline C & -2.851732 & 1.479438 & 1.141986 \\
\hline C & -1.176068 & 3.191118 & 0.530731 \\
\hline $\mathrm{H}$ & -4.578240 & 1.106423 & -0.082242 \\
\hline C & -4.148675 & -0.701289 & 0.969506 \\
\hline 0 & -1.712699 & -0.754486 & -2.525666 \\
\hline C & -3.910545 & -1.534226 & -2.108347 \\
\hline $\mathrm{H}$ & -2.226239 & 0.862797 & 1.810418 \\
\hline C & -3.726770 & 2.382179 & 1.964496 \\
\hline 0 & -1.010945 & 3.486314 & 1.699118 \\
\hline C & -0.508321 & 3.953408 & -0.569569 \\
\hline C & -3.293252 & -1.789881 & 1.161800 \\
\hline C & -5.439026 & -0.740966 & 1.501218 \\
\hline C & -3.652438 & -2.796350 & -2.655278 \\
\hline C & -5.238710 & -1.155736 & -1.890134 \\
\hline C & -3.999410 & 2.050927 & 3.292311 \\
\hline C & -4.348928 & 3.497986 & 1.396898 \\
\hline C & -1.039966 & 4.070826 & -1.858608 \\
\hline C & 0.671526 & 4.640529 & -0.271780 \\
\hline C & -3.736263 & -2.913709 & 1.853499 \\
\hline $\mathrm{H}$ & -2.277373 & -1.744955 & 0.765427 \\
\hline C & -5.877649 & -1.861016 & 2.205163 \\
\hline $\mathrm{H}$ & -6.109152 & 0.111337 & 1.358514 \\
\hline C & -4.691232 & -3.678368 & -2.919538 \\
\hline $\mathrm{H}$ & -2.618237 & -3.065060 & -2.876975 \\
\hline C & -6.276064 & -2.036032 & -2.173251 \\
\hline $\mathrm{H}$ & -5.478095 & -0.158985 & -1.519168 \\
\hline C & -4.879322 & 2.825080 & 4.046433 \\
\hline $\mathrm{H}$ & -3.519870 & 1.175005 & 3.736534 \\
\hline C & -5.221074 & 4.277163 & 2.151616 \\
\hline $\mathrm{H}$ & -4.139307 & 3.762779 & 0.357022 \\
\hline C & -0.370347 & 4.798866 & -2.836035 \\
\hline $\mathrm{H}$ & -2.000742 & 3.609790 & -2.095987 \\
\hline C & 1.345529 & 5.359707 & -1.253263 \\
\hline $\mathrm{H}$ & 1.037228 & 4.622388 & 0.756173 \\
\hline C & -5.029741 & -2.953666 & 2.375150 \\
\hline $\mathrm{H}$ & -3.065995 & -3.767553 & 1.982115 \\
\hline $\mathrm{H}$ & -6.890319 & -1.882852 & 2.614194 \\
\hline C & -6.023150 & -3.319247 & -2.672952 \\
\hline H & -4.469912 & -4.665624 & -3.333530 \\
\hline $\mathrm{H}$ & -7.308343 & -1.720390 & -2.002189 \\
\hline C & -5.490110 & 3.941193 & 3.478645 \\
\hline $\mathrm{H}$ & -5.086303 & 2.555924 & 5.084729 \\
\hline $\mathrm{H}$ & -5.694713 & 5.153120 & 1.702294 \\
\hline C & 0.844323 & 5.442019 & -2.559572 \\
\hline $\mathrm{H}$ & -0.805922 & 4.882232 & -3.835369 \\
\hline $\mathrm{H}$ & 2.268613 & 5.888673 & -0.999602 \\
\hline $\mathrm{H}$ & -5.377402 & -3.837926 & 2.914412 \\
\hline $\mathrm{H}$ & -6.175906 & 4.551935 & 4.070228 \\
\hline C & 1.547988 & 6.241682 & -3.614495 \\
\hline $\mathrm{H}$ & 1.085783 & 7.237336 & -3.717500 \\
\hline $\mathrm{H}$ & 2.607787 & 6.397042 & -3.368840 \\
\hline $\mathrm{H}$ & 1.486592 & 5.755390 & -4.599040 \\
\hline C & -7.142501 & -4.284554 & -2.920149 \\
\hline $\mathrm{H}$ & -6.891800 & -5.000597 & -3.715526 \\
\hline $\mathrm{H}$ & -7.356770 & -4.867843 & -2.008930 \\
\hline $\mathrm{H}$ & -8.070183 & -3.765539 & -3.20021 \\
\hline
\end{tabular}


$\operatorname{TS}(1-2)^{S}$

Lowest frequency $=-415.4956 \mathrm{~cm}^{-1}$

Charge $=1$, Multiplicity $=1$

131

\begin{tabular}{|c|c|c|c|}
\hline $\mathrm{N}$ & 2.296624 & -1.956097 & 0.983678 \\
\hline $\mathrm{N}$ & 1.791440 & -1.782657 & -1.231532 \\
\hline C & 3.670022 & -2.814628 & 4.782260 \\
\hline C & 3.362943 & -3.915968 & 3.957006 \\
\hline $\mathrm{H}$ & 3.506777 & -4.926921 & 4.344981 \\
\hline C & 2.886859 & -3.755827 & 2.664021 \\
\hline $\mathrm{H}$ & 2.682009 & -4.627259 & 2.043567 \\
\hline C & 2.717035 & -2.445843 & 2.196063 \\
\hline C & 3.052965 & -1.323066 & 3.005127 \\
\hline C & 3.523211 & -1.521414 & 4.316594 \\
\hline $\mathrm{H}$ & 3.763901 & -0.666254 & 4.950541 \\
\hline C & 2.837555 & -0.140084 & 2.233317 \\
\hline C & 2.367340 & -0.533176 & 0.974800 \\
\hline C & 0.831671 & 1.483889 & 4.903485 \\
\hline $\mathrm{H}$ & 1.042293 & 2.454467 & 5.373068 \\
\hline C & 0.538329 & 1.517466 & 3.437918 \\
\hline $\mathrm{H}$ & 0.280972 & 0.505811 & 3.083834 \\
\hline $\mathrm{H}$ & -0.346148 & 2.147929 & 3.247229 \\
\hline C & 1.692487 & 2.082519 & 2.609394 \\
\hline $\mathrm{H}$ & 1.909143 & 3.113843 & 2.934733 \\
\hline $\mathrm{H}$ & 1.385041 & 2.156685 & 1.553583 \\
\hline C & 2.984441 & 1.264875 & 2.713966 \\
\hline $\mathrm{H}$ & 3.777741 & 1.769970 & 2.137482 \\
\hline $\mathrm{H}$ & 3.323463 & 1.253813 & 3.760194 \\
\hline C & 0.853087 & 0.386261 & 5.662233 \\
\hline $\mathrm{H}$ & 0.644899 & -0.602052 & 5.238613 \\
\hline $\mathrm{H}$ & 1.060804 & 0.433662 & 6.734561 \\
\hline C & 1.760404 & -2.565374 & -0.130173 \\
\hline C & 1.162049 & -3.822856 & -0.166455 \\
\hline $\mathrm{H}$ & 1.099860 & -4.430485 & 0.733801 \\
\hline C & 0.592269 & -4.253215 & -1.359173 \\
\hline $\mathrm{H}$ & 0.108911 & -5.232823 & -1.396858 \\
\hline C & 0.593536 & -3.437989 & -2.494444 \\
\hline C & 1.209673 & -2.194311 & -2.361687 \\
\hline $\mathrm{H}$ & 1.216106 & -1.483381 & -3.188740 \\
\hline C & -0.087681 & -3.807494 & -3.773143 \\
\hline $\mathrm{H}$ & -0.503267 & -4.822742 & -3.728982 \\
\hline $\mathrm{H}$ & 0.601934 & -3.757548 & -4.629311 \\
\hline $\mathrm{H}$ & -0.908476 & -3.096688 & -3.958823 \\
\hline $\mathrm{H}$ & 4.033736 & -2.991604 & 5.796128 \\
\hline $\mathrm{Ru}$ & 2.708788 & 0.082987 & -1.045849 \\
\hline $\mathrm{C}$ & 3.368743 & 1.854779 & -2.200032 \\
\hline C & 4.697506 & 0.699589 & -0.474283 \\
\hline C & 3.484854 & 0.695989 & -3.020423 \\
\hline C & 3.990774 & 1.850925 & -0.924168 \\
\hline C & 4.823902 & -0.468848 & -1.267814 \\
\hline $\mathrm{H}$ & 5.097249 & 0.694126 & 0.540090 \\
\hline C & 4.164836 & -0.444093 & -2.534526 \\
\hline $\mathrm{H}$ & 2.961788 & 0.657312 & -3.977302 \\
\hline $\mathrm{H}$ & 3.864976 & 2.708513 & -0.261977 \\
\hline H & 4.142327 & -1.362937 & -3.127851 \\
\hline C & 2.534945 & 3.007774 & -2.643584 \\
\hline $\mathrm{H}$ & 1.684456 & 2.670452 & -3.248744 \\
\hline
\end{tabular}

\begin{tabular}{|c|c|c|c|}
\hline $\mathrm{H}$ & 2.141937 & 3.572172 & -1.789697 \\
\hline $\mathrm{H}$ & 3.149632 & 3.688570 & -3.254306 \\
\hline C & 5.588971 & -1.703105 & -0.844374 \\
\hline C & 5.930218 & -1.738683 & 0.637659 \\
\hline C & 6.850594 & -1.833966 & -1.701450 \\
\hline $\mathrm{H}$ & 4.933536 & -2.564175 & -1.067009 \\
\hline $\mathrm{H}$ & 5.043010 & -1.616630 & 1.272844 \\
\hline $\mathrm{H}$ & 6.391262 & -2.702966 & 0.894204 \\
\hline $\mathrm{H}$ & 6.656767 & -0.954206 & 0.902346 \\
\hline $\mathrm{H}$ & 6.619951 & -1.866077 & -2.776798 \\
\hline $\mathrm{H}$ & 7.528937 & -0.983873 & -1.528337 \\
\hline $\mathrm{H}$ & 7.393805 & -2.755764 & -1.446744 \\
\hline $\mathrm{H}$ & 1.212038 & -0.122164 & 0.774396 \\
\hline 0 & 0.798058 & 0.867943 & -1.339255 \\
\hline C & -0.189708 & 0.646182 & -0.594227 \\
\hline 0 & -0.139025 & 0.059001 & 0.507791 \\
\hline C & -1.559619 & 1.191268 & -1.041714 \\
\hline $\mathrm{N}$ & -2.544273 & 0.134954 & -1.082204 \\
\hline $\mathrm{N}$ & -2.111457 & 2.094820 & -0.049479 \\
\hline $\mathrm{H}$ & -1.425703 & 1.649194 & -2.030806 \\
\hline C & -3.533126 & 0.229451 & -0.006190 \\
\hline C & -2.377248 & -0.881426 & -1.970064 \\
\hline C & -2.945906 & 1.343725 & 0.886475 \\
\hline C & -1.410429 & 3.182009 & 0.451417 \\
\hline $\mathrm{H}$ & -4.492029 & 0.585505 & -0.414390 \\
\hline C & -3.770409 & -1.059747 & 0.740973 \\
\hline 0 & -1.348247 & -0.966356 & -2.629579 \\
\hline C & -3.501085 & -1.850831 & -2.095161 \\
\hline $\mathrm{H}$ & -2.313452 & 0.887937 & 1.665301 \\
\hline C & -4.026765 & 2.159353 & 1.541719 \\
\hline 0 & -1.435031 & 3.446300 & 1.636302 \\
\hline C & -0.680506 & 4.054597 & -0.513222 \\
\hline C & -2.697566 & -1.793039 & 1.261468 \\
\hline C & -5.070759 & -1.529493 & 0.925229 \\
\hline C & -3.275540 & -3.207207 & -1.848477 \\
\hline C & -4.777550 & -1.425641 & -2.466533 \\
\hline C & -4.367138 & 1.907487 & 2.870945 \\
\hline C & -4.749826 & 3.111432 & 0.817902 \\
\hline C & -1.083838 & 4.239760 & -1.838563 \\
\hline C & 0.368484 & 4.835743 & -0.010975 \\
\hline C & -2.928354 & -2.985679 & 1.940117 \\
\hline $\mathrm{H}$ & -1.678462 & -1.424956 & 1.122184 \\
\hline C & -5.301558 & -2.723878 & 1.606109 \\
\hline $\mathrm{H}$ & -5.911072 & -0.961921 & 0.518336 \\
\hline C & -4.323581 & -4.114067 & -1.931842 \\
\hline $\mathrm{H}$ & -2.278264 & -3.538735 & -1.552439 \\
\hline C & -5.816192 & -2.345443 & -2.576157 \\
\hline $\mathrm{H}$ & -4.961702 & -0.369293 & -2.678502 \\
\hline C & -5.417903 & 2.597834 & 3.471908 \\
\hline $\mathrm{H}$ & -3.799041 & 1.167342 & 3.440260 \\
\hline C & -5.793665 & 3.808388 & 1.419927 \\
\hline $\mathrm{H}$ & -4.481537 & 3.316721 & -0.222083 \\
\hline C & -0.434809 & 5.167635 & -2.649080 \\
\hline $\mathrm{H}$ & -1.940703 & 3.688963 & -2.232006 \\
\hline C & 1.015045 & 5.755689 & -0.825007 \\
\hline $\mathrm{H}$ & 0.643967 & 4.720391 & 1.039165 \\
\hline C & -4.230599 & -3.457343 & 2.111226 \\
\hline $\mathrm{H}$ & -2.084603 & -3.551695 & 2.343193 \\
\hline $\mathrm{H}$ & -6.324508 & -3.083212 & 1.739099 \\
\hline C & -5.613733 & -3.700405 & -2.292482 \\
\hline $\mathrm{H}$ & -4.143311 & -5.168743 & -1.707357 \\
\hline
\end{tabular}




$\begin{array}{rrrr}\text { H } & -6.808884 & -2.003168 & -2.879830 \\ \mathrm{C} & -6.131964 & 3.550889 & 2.748549 \\ \mathrm{H} & -5.675006 & 2.394953 & 4.514005 \\ \mathrm{H} & -6.346618 & 4.558941 & 0.850265 \\ \mathrm{C} & 0.625712 & 5.939444 & -2.161020 \\ \mathrm{H} & -0.772063 & 5.310465 & -3.678978 \\ \mathrm{H} & 1.830602 & 6.359028 & -0.416682 \\ \mathrm{H} & -4.410608 & -4.394657 & 2.642843 \\ \mathrm{H} & -6.950993 & 4.097812 & 3.221137 \\ \mathrm{C} & 1.307746 & 6.958399 & -3.023164 \\ \mathrm{H} & 0.988751 & 7.976746 & -2.746807 \\ \mathrm{H} & 2.400836 & 6.921984 & -2.901605 \\ \mathrm{H} & 1.072335 & 6.815290 & -4.086632 \\ \mathrm{C} & -6.745131 & -4.682773 & -2.343329 \\ \mathrm{H} & -6.436382 & -5.630155 & -2.809295 \\ \mathrm{H} & -7.095795 & -4.921327 & -1.325475 \\ \mathrm{H} & -7.602255 & -4.286522 & -2.904811\end{array}$

\section{I-2 $2^{S}$}

Lowest frequency $=12.0604 \mathrm{~cm}^{-1}$ Charge $=1$, Multiplicity $=1$

131

\begin{tabular}{|c|c|c|c|}
\hline $\mathrm{N}$ & 2.159101 & -1.720774 & 1.683592 \\
\hline $\mathrm{N}$ & 2.747848 & 0.434929 & 1.259953 \\
\hline C & 1.132890 & -5.541359 & 2.899094 \\
\hline C & 1.460445 & -4.637483 & 3.920168 \\
\hline $\mathrm{H}$ & 1.449845 & -4.972097 & 4.959629 \\
\hline C & 1.816792 & -3.321812 & 3.638644 \\
\hline $\mathrm{H}$ & 2.106576 & -2.658747 & 4.451995 \\
\hline C & 1.827426 & -2.924370 & 2.299613 \\
\hline C & 1.515143 & -3.830078 & 1.256430 \\
\hline C & 1.165239 & -5.149906 & 1.566866 \\
\hline $\mathrm{H}$ & 0.921043 & -5.857428 & 0.772733 \\
\hline C & 1.687359 & -3.142434 & -0.006832 \\
\hline C & 2.107159 & -1.855361 & 0.269632 \\
\hline C & -1.505226 & -5.082031 & -1.200398 \\
\hline $\mathrm{H}$ & -1.552345 & -5.546798 & -2.194797 \\
\hline C & -1.155406 & -3.627635 & -1.179124 \\
\hline $\mathrm{H}$ & -1.051559 & -3.291537 & -0.133940 \\
\hline $\mathrm{H}$ & -2.001159 & -3.059083 & -1.599958 \\
\hline C & 0.094927 & -3.272520 & -1.982504 \\
\hline $\mathrm{H}$ & 0.009642 & -3.709387 & -2.992436 \\
\hline $\mathrm{H}$ & 0.126912 & -2.181832 & -2.135421 \\
\hline C & 1.405772 & -3.748465 & -1.348153 \\
\hline $\mathrm{H}$ & 2.232343 & -3.532837 & -2.042298 \\
\hline $\mathrm{H}$ & 1.379925 & -4.844546 & -1.244508 \\
\hline C & -1.788339 & -5.827372 & -0.130560 \\
\hline $\mathrm{H}$ & -1.753467 & -5.408375 & 0.880762 \\
\hline $\mathrm{H}$ & -2.070138 & -6.879440 & -0.223630 \\
\hline C & 2.354752 & -0.461671 & 2.188968 \\
\hline C & 2.121925 & -0.057327 & 3.508783 \\
\hline $\mathrm{H}$ & 1.759436 & -0.768769 & 4.245515 \\
\hline C & 2.308025 & 1.272390 & 3.843306 \\
\hline $\mathrm{H}$ & 2.107558 & 1.598739 & 4.866800 \\
\hline C & 2.729657 & 2.207846 & 2.887142 \\
\hline C & 2.935455 & 1.718065 & 1.604915 \\
\hline
\end{tabular}

\begin{tabular}{|c|c|c|c|}
\hline $\mathrm{H}$ & 3.244337 & 2.377763 & 0.793329 \\
\hline C & 2.871533 & 3.663365 & 3.201029 \\
\hline $\mathrm{H}$ & 1.899625 & 4.162636 & 3.055230 \\
\hline $\mathrm{H}$ & 3.187273 & 3.821298 & 4.241416 \\
\hline $\mathrm{H}$ & 3.606406 & 4.151581 & 2.545254 \\
\hline $\mathrm{H}$ & 0.862640 & -6.567606 & 3.156349 \\
\hline $\mathrm{Ru}$ & 2.878239 & -0.232756 & -0.703776 \\
\hline C & 3.223979 & 0.467759 & -2.784376 \\
\hline C & 4.198425 & -1.557908 & -1.769986 \\
\hline C & 4.035583 & 1.263085 & -1.928669 \\
\hline C & 3.335986 & -0.950297 & -2.721384 \\
\hline C & 4.970594 & -0.788896 & -0.858200 \\
\hline $\mathrm{H}$ & 4.202185 & -2.644675 & -1.679747 \\
\hline C & 4.849988 & 0.633278 & -0.963473 \\
\hline $\mathrm{H}$ & 3.930597 & 2.349430 & -1.942111 \\
\hline $\mathrm{H}$ & 2.703127 & -1.568593 & -3.359020 \\
\hline $\mathrm{H}$ & 5.372302 & 1.250349 & -0.226347 \\
\hline C & 2.222184 & 1.109602 & -3.689187 \\
\hline $\mathrm{H}$ & 1.935840 & 2.104263 & -3.322037 \\
\hline $\mathrm{H}$ & 1.314591 & 0.496082 & -3.773189 \\
\hline $\mathrm{H}$ & 2.656704 & 1.220640 & -4.695628 \\
\hline C & 5.901246 & -1.392298 & 0.168291 \\
\hline C & 5.517775 & -2.802109 & 0.597697 \\
\hline C & 7.336668 & -1.337185 & -0.359596 \\
\hline $\mathrm{H}$ & 5.838292 & -0.739212 & 1.056623 \\
\hline $\mathrm{H}$ & 4.474731 & -2.858255 & 0.939275 \\
\hline $\mathrm{H}$ & 6.165242 & -3.130578 & 1.423135 \\
\hline $\mathrm{H}$ & 5.651753 & -3.527967 & -0.219737 \\
\hline $\mathrm{H}$ & 7.637671 & -0.312512 & -0.625153 \\
\hline $\mathrm{H}$ & 7.445582 & -1.963206 & -1.259232 \\
\hline $\mathrm{H}$ & 8.041082 & -1.710987 & 0.397998 \\
\hline $\mathrm{H}$ & 0.373894 & -1.249788 & 0.145976 \\
\hline 0 & 0.994459 & 0.695308 & -0.959923 \\
\hline C & -0.141650 & 0.395835 & -0.597138 \\
\hline 0 & -0.434215 & -0.653777 & 0.097565 \\
\hline C & -1.271918 & 1.384310 & -0.882309 \\
\hline $\mathrm{N}$ & -2.563192 & 0.760241 & -0.907633 \\
\hline $\mathrm{N}$ & -1.380707 & 2.341190 & 0.192854 \\
\hline $\mathrm{H}$ & -1.015457 & 1.841197 & -1.845462 \\
\hline C & -3.351038 & 1.100785 & 0.275755 \\
\hline C & -2.739715 & -0.121680 & -1.943940 \\
\hline C & -2.295664 & 1.792597 & 1.187138 \\
\hline C & -0.365532 & 3.229074 & 0.520784 \\
\hline $\mathrm{H}$ & -4.097311 & 1.871620 & 0.019766 \\
\hline C & -4.047753 & -0.041258 & 0.967987 \\
\hline 0 & -1.750123 & -0.401891 & -2.618173 \\
\hline C & -4.074011 & -0.692682 & -2.237735 \\
\hline $\mathrm{H}$ & -1.795419 & 1.019131 & 1.796445 \\
\hline C & -2.920325 & 2.811426 & 2.097068 \\
\hline 0 & -0.076786 & 3.460195 & 1.676681 \\
\hline C & 0.314862 & 3.907641 & -0.623050 \\
\hline C & -3.526488 & -1.337454 & 0.968114 \\
\hline C & -5.229323 & 0.219915 & 1.666635 \\
\hline C & -4.110546 & -1.877486 & -2.983939 \\
\hline C & -5.283872 & -0.091293 & -1.873444 \\
\hline C & -3.137135 & 2.496273 & 3.438670 \\
\hline C & -3.368648 & 4.039378 & 1.601113 \\
\hline C & -0.320865 & 4.176272 & -1.841303 \\
\hline C & 1.625610 & 4.355559 & -0.442297 \\
\hline C & -4.187614 & -2.360519 & 1.642086 \\
\hline $\mathrm{H}$ & -2.604924 & -1.552356 & 0.427565 \\
\hline
\end{tabular}




$\begin{array}{rrrr}\text { C } & -5.884062 & -0.801194 & 2.352119 \\ \text { H } & -5.642198 & 1.232452 & 1.671563 \\ \text { C } & -5.321342 & -2.474093 & -3.304703 \\ \text { H } & -3.166954 & -2.313683 & -3.315510 \\ \text { C } & -6.493341 & -0.685770 & -2.210352 \\ \text { H } & -5.297580 & 0.859928 & -1.342710 \\ \text { C } & -3.792575 & 3.395893 & 4.277348 \\ \text { H } & -2.792588 & 1.534882 & 3.827884 \\ \text { C } & -4.015706 & 4.942159 & 2.439558 \\ \text { H } & -3.200023 & 4.294852 & 0.551312 \\ \text { C } & 0.362067 & 4.818127 & -2.867971 \\ \text { H } & -1.369711 & 3.905053 & -1.981882 \\ \text { C } & 2.310385 & 4.987209 & -1.474460 \\ \text { H } & 2.091494 & 4.215480 & 0.532923 \\ \mathrm{C} & -5.367544 & -2.095565 & 2.336475 \\ \text { H } & -3.780472 & -3.374173 & 1.615393 \\ \text { H } & -6.808144 & -0.585252 & 2.893013 \\ \text { C } & -6.536880 & -1.896043 & -2.913628 \\ \text { H } & -5.328930 & -3.405359 & -3.876818 \\ \text { H } & -7.427924 & -0.198709 & -1.921379 \\ \text { C } & -4.231250 & 4.621117 & 3.780149 \\ \text { H } & -3.958691 & 3.138764 & 5.325896 \\ \text { H } & -4.354148 & 5.903259 & 2.045836 \\ \text { C } & 1.696176 & 5.220367 & -2.712307 \\ \text { H } & -0.153765 & 5.022918 & -3.809745 \\ \text { H } & 3.337515 & 5.327224 & -1.314538 \\ \text { H } & -5.887878 & -2.899712 & 2.861664 \\ \text { H } & -4.740796 & 5.329174 & 4.437551 \\ \text { C } & 2.418618 & 5.927631 & -3.818718 \\ \text { H } & 3.508562 & 5.884342 & -3.686424 \\ \text { H } & 2.169887 & 5.500776 & -4.801235 \\ \text { H } & 2.132987 & 6.992124 & -3.847855 \\ \mathrm{C} & -7.842644 & -2.561060 & -3.224897 \\ \text { H } & -7.790460 & -3.137287 & -4.159494 \\ \text { H } & -8.116290 & -3.265238 & -2.421363 \\ \text { H } & -8.659196 & -1.830539 & -3.311316\end{array}$

\section{I-3 $3^{S}$}

Lowest frequency $=22.3807 \mathrm{~cm}^{-1}$ Charge $=1$, Multiplicity $=1$

65

$\begin{array}{rrrr}\mathrm{N} & -1.545409 & 0.660823 & -0.164056 \\ \mathrm{~N} & 0.503749 & 1.474491 & -0.714128 \\ \mathrm{C} & -5.468058 & -0.623982 & 0.236097 \\ \mathrm{C} & -5.279004 & 0.762912 & 0.196486 \\ \mathrm{H} & -6.140869 & 1.428336 & 0.276352 \\ \mathrm{C} & -4.006489 & 1.316373 & 0.064851 \\ \mathrm{H} & -3.895483 & 2.399371 & 0.064992 \\ \mathrm{C} & -2.925132 & 0.442958 & -0.035218 \\ \mathrm{C} & -3.097287 & -0.963944 & 0.028331 \\ \mathrm{C} & -4.385195 & -1.493200 & 0.158219 \\ \mathrm{H} & -4.538403 & -2.573751 & 0.203357 \\ \mathrm{C} & -1.788620 & -1.582739 & -0.048932 \\ \mathrm{C} & -0.871907 & -0.576692 & -0.130194 \\ \mathrm{C} & 0.458422 & -1.281873 & -2.152703 \\ \mathrm{H} & -0.411395 & -0.707941 & -2.490175\end{array}$

\begin{tabular}{rrrr}
$\mathrm{C}$ & 0.259027 & -2.777856 & -2.175394 \\
$\mathrm{H}$ & 1.081275 & -3.272862 & -1.635405 \\
$\mathrm{H}$ & 0.380255 & -3.079369 & -3.232502 \\
$\mathrm{C}$ & -1.092751 & -3.320420 & -1.707113 \\
$\mathrm{H}$ & -1.884611 & -2.923364 & -2.364417 \\
$\mathrm{H}$ & -1.082424 & -4.410183 & -1.870154 \\
$\mathrm{C}$ & -1.489380 & -3.032984 & -0.254696 \\
$\mathrm{H}$ & -0.694525 & -3.378783 & 0.427170 \\
$\mathrm{H}$ & -2.374804 & -3.635431 & -0.003132 \\
$\mathrm{C}$ & 1.700024 & -0.654400 & -2.286939 \\
$\mathrm{H}$ & 2.611158 & -1.252935 & -2.365293 \\
$\mathrm{H}$ & 1.753636 & 0.331411 & -2.756607 \\
$\mathrm{C}$ & -0.810864 & 1.747184 & -0.541516 \\
$\mathrm{C}$ & -1.299199 & 3.041656 & -0.776459 \\
$\mathrm{H}$ & -2.356947 & 3.257164 & -0.658060 \\
$\mathrm{C}$ & -0.420297 & 4.027895 & -1.178516 \\
$\mathrm{H}$ & -0.799238 & 5.035906 & -1.365925 \\
$\mathrm{C}$ & 0.947019 & 3.751971 & -1.358498 \\
$\mathrm{C}$ & 1.344078 & 2.447476 & -1.112181 \\
$\mathrm{H}$ & 2.385980 & 2.146111 & -1.241312 \\
$\mathrm{C}$ & 1.919235 & 4.801283 & -1.800101 \\
$\mathrm{H}$ & 1.632086 & 5.220261 & -2.776814 \\
$\mathrm{H}$ & 1.952265 & 5.637849 & -1.085102 \\
$\mathrm{H}$ & -0.727219 & -0.506155 & 3.924774 \\
$\mathrm{H}$ & 2.936354 & 4.397018 & -1.894009 \\
$\mathrm{H}$ & 1.571052 & 0.342719 & 4.737728 \\
$\mathrm{H}$ & -6.478388 & -1.025298 & 0.338736 \\
$\mathrm{Ru}$ & 1.134958 & -0.415616 & -0.195754 \\
$\mathrm{C}$ & 2.977816 & -1.696501 & 0.307390 \\
$\mathrm{C}$ & 1.053759 & -1.378732 & 1.799780 \\
$\mathrm{C}$ & 3.325279 & -0.327722 & 0.514281 \\
$\mathrm{C}$ & 1.846798 & -2.215489 & 0.982089 \\
$\mathrm{C}$ & 1.365023 & -0.006435 & 1.995642 \\
$\mathrm{H}$ & 0.147273 & -1.788625 & 2.246445 \\
$\mathrm{C}$ & 2.525513 & 0.486810 & 1.329328 \\
$\mathrm{H}$ & 4.196173 & 0.091438 & 0.006884 \\
$\mathrm{H}$ & 1.574386 & -3.263367 & 0.852169 \\
$\mathrm{H}$ & 2.771704 & 1.547917 & 1.424606 \\
$\mathrm{C}$ & 3.845917 & -2.585978 & -0.523317 \\
$\mathrm{H}$ & 4.424055 & -2.020800 & -1.266201 \\
$\mathrm{H}$ & 3.264919 & -3.362349 & -1.038699 \\
$\mathrm{H}$ & 4.565494 & -3.096224 & 0.137059 \\
\hline & -0.564533 & 0.914954 & 2.890942 \\
$\mathrm{H}$ & -1.3840734 & 0.372739 & 3.265809 \\
$\mathrm{H}$ & 1.248881 & 4.140670 \\
$\mathrm{H}$ & -1.962755 & 4.775027
\end{tabular}

\section{TS(3-4)}

Lowest frequency $=-280.3059 \mathrm{~cm}^{-1}$ Charge $=1$, Multiplicity $=1$

65

$\begin{array}{llll}\mathrm{N} & -1.445371 & 0.747554 & -0.446403\end{array}$ 


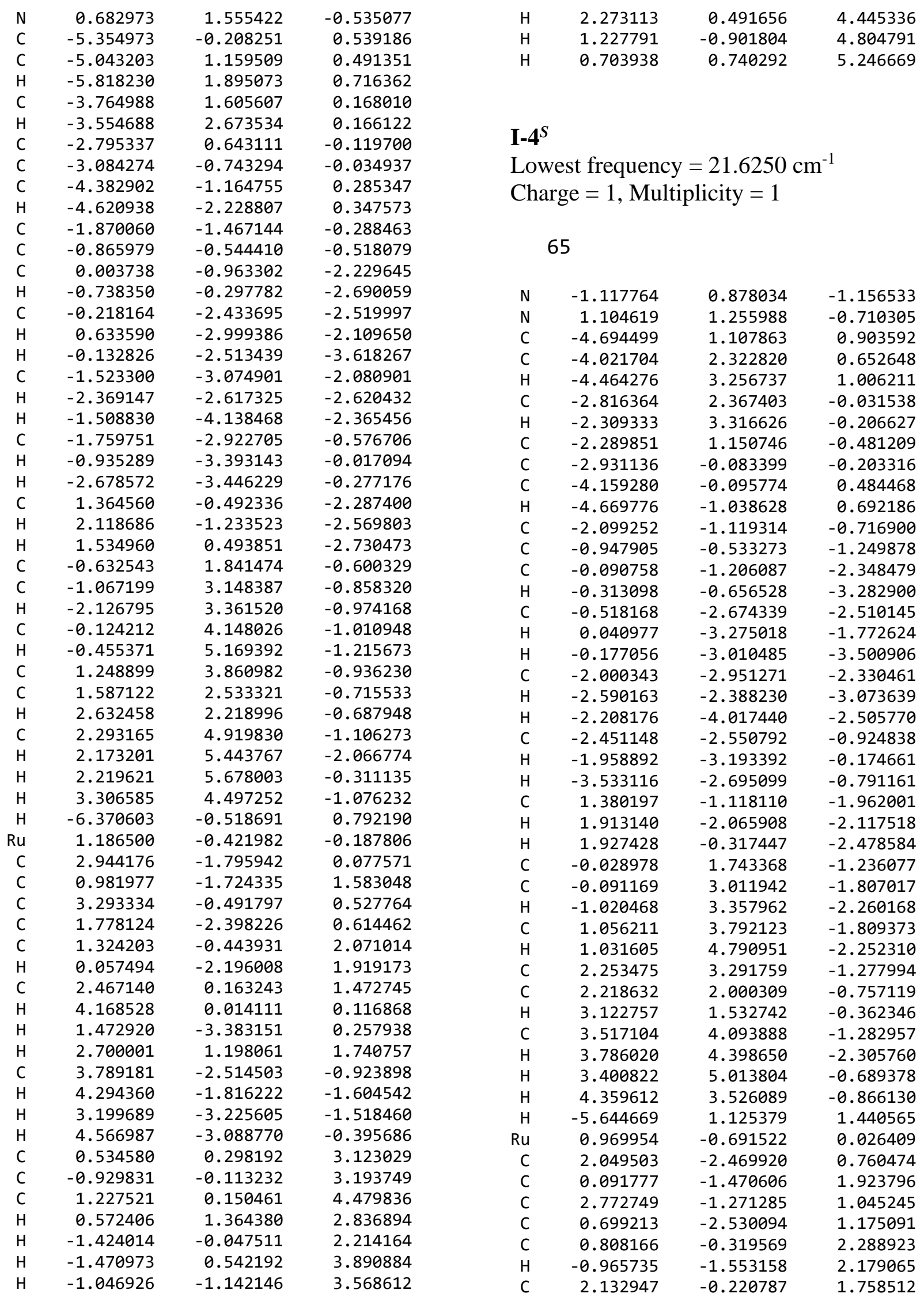




$\begin{array}{rrrr}\text { H } & 3.800165 & -1.152972 & 0.696561 \\ \text { H } & 0.100049 & -3.400060 & 0.900745 \\ \text { H } & 2.680249 & 0.714770 & 1.908207 \\ \mathrm{C} & 2.691370 & -3.599275 & 0.023294 \\ \mathrm{H} & 3.440112 & -3.238636 & -0.694796 \\ \mathrm{H} & 1.950618 & -4.204951 & -0.515710 \\ \mathrm{H} & 3.205352 & -4.257967 & 0.741409 \\ \mathrm{C} & 0.240916 & 0.808905 & 3.117039 \\ \mathrm{C} & -1.279899 & 0.829727 & 3.160786 \\ \mathrm{C} & 0.840190 & 0.760703 & 4.524910 \\ \mathrm{H} & 0.579607 & 1.744676 & 2.636833 \\ \mathrm{H} & -1.719335 & 0.837044 & 2.153044 \\ \mathrm{H} & -1.630824 & 1.729230 & 3.686279 \\ \mathrm{H} & -1.681356 & -0.038240 & 3.707072 \\ \mathrm{H} & 1.939992 & 0.789358 & 4.504314 \\ \mathrm{H} & 0.538625 & -0.162344 & 5.044221 \\ \mathrm{H} & 0.491214 & 1.615313 & 5.123263\end{array}$

\section{I-5 5}

Lowest frequency $=6.0672 \mathrm{~cm}^{-1}$ Charge $=1$, Multiplicity $=1$

131

$\begin{array}{lrrr}\text { N } & 3.800920 & -1.405008 & 1.262727 \\ \text { N } & 2.249757 & 0.377521 & 1.153368 \\ \text { C } & 7.645867 & -2.800995 & 1.900503 \\ \text { C } & 7.364265 & -1.505937 & 2.365042 \\ \text { H } & 8.151802 & -0.925210 & 2.849926 \\ \text { C } & 6.099121 & -0.944979 & 2.215475 \\ \text { H } & 5.890162 & 0.064816 & 2.575451 \\ \text { C } & 5.120702 & -1.718964 & 1.589435 \\ \mathrm{C} & 5.387770 & -3.023372 & 1.106123 \\ \mathrm{C} & 6.670318 & -3.563435 & 1.269617 \\ \text { H } & 6.899879 & -4.565846 & 0.901286 \\ \mathrm{C} & 4.173480 & -3.503730 & 0.496912 \\ \mathrm{C} & 3.249233 & -2.500889 & 0.579920 \\ \mathrm{C} & 1.874442 & -2.511602 & 0.010576 \\ \mathrm{H} & 1.173722 & -2.275938 & 0.839849 \\ \mathrm{C} & 1.524446 & -3.932168 & -0.500794 \\ \mathrm{H} & 1.714511 & -3.950968 & -1.587199 \\ \mathrm{H} & 0.441687 & -4.096621 & -0.383740 \\ \mathrm{C} & 2.317313 & -5.066082 & 0.139278 \\ \mathrm{H} & 2.090638 & -5.128148 & 1.217435 \\ \mathrm{H} & 2.008196 & -6.024288 & -0.306214 \\ \mathrm{C} & 3.821345 & -4.853745 & -0.035603 \\ \mathrm{H} & 4.098893 & -4.953125 & -1.101065 \\ \mathrm{H} & 4.389829 & -5.629250 & 0.501576 \\ \mathrm{C} & 1.740592 & -1.508557 & -1.150392 \\ \mathrm{H} & 2.347406 & -1.925253 & -1.966595 \\ \mathrm{H} & 0.706001 & -1.530860 & -1.533726 \\ \mathrm{C} & 3.062569 & -0.402782 & 1.890872 \\ \mathrm{C} & 3.165705 & -0.220492 & 3.274343 \\ \mathrm{H} & 3.810980 & -0.889043 & 3.844956 \\ \mathrm{C} & 2.435523 & 0.779958 & 3.892773 \\ \mathrm{H} & 2.508587 & 0.918036 & 4.974472 \\ \mathrm{C} & 1.581888 & 1.595940 & 3.138111 \\ \mathrm{C} & 1.527332 & 1.333020 & 1.773849\end{array}$

\begin{tabular}{|c|c|c|c|}
\hline $\mathrm{H}$ & 0.883576 & 1.932581 & 1.126705 \\
\hline C & 0.774374 & 2.702538 & 3.741156 \\
\hline $\mathrm{H}$ & -0.236973 & 2.747236 & 3.310562 \\
\hline $\mathrm{H}$ & 0.686597 & 2.578751 & 4.828825 \\
\hline $\mathrm{H}$ & 1.257489 & 3.677086 & 3.560134 \\
\hline $\mathrm{H}$ & 8.649558 & -3.209889 & 2.033903 \\
\hline $\mathrm{Ru}$ & 2.349813 & 0.500166 & -0.962876 \\
\hline C & 4.148334 & 0.017966 & -2.117692 \\
\hline C & 3.933183 & 2.104122 & -0.779654 \\
\hline C & 3.121173 & 0.520959 & -2.980008 \\
\hline C & 4.489469 & 0.801473 & -0.990249 \\
\hline C & 2.945190 & 2.619160 & -1.624710 \\
\hline $\mathrm{H}$ & 4.230174 & 2.657463 & 0.111906 \\
\hline C & 2.504243 & 1.756747 & -2.689372 \\
\hline $\mathrm{H}$ & 2.755359 & -0.085785 & -3.810318 \\
\hline $\mathrm{H}$ & 5.211460 & 0.403480 & -0.274214 \\
\hline $\mathrm{H}$ & 1.636790 & 2.058213 & -3.281984 \\
\hline C & 4.842314 & -1.276866 & -2.385300 \\
\hline $\mathrm{H}$ & 4.205385 & -1.976697 & -2.942099 \\
\hline $\mathrm{H}$ & 5.164652 & -1.759677 & -1.452952 \\
\hline $\mathrm{H}$ & 5.736646 & -1.081808 & -2.998552 \\
\hline C & 2.276449 & 3.957190 & -1.415377 \\
\hline C & 2.643066 & 4.609290 & -0.090532 \\
\hline C & 2.583690 & 4.889607 & -2.588817 \\
\hline $\mathrm{H}$ & 1.188303 & 3.766806 & -1.414320 \\
\hline $\mathrm{H}$ & 2.390306 & 3.969809 & 0.769129 \\
\hline $\mathrm{H}$ & 2.098004 & 5.554763 & 0.027817 \\
\hline $\mathrm{H}$ & 3.717667 & 4.845597 & -0.039496 \\
\hline $\mathrm{H}$ & 2.292611 & 4.446796 & -3.552893 \\
\hline $\mathrm{H}$ & 3.658851 & 5.123116 & -2.636177 \\
\hline $\mathrm{H}$ & 2.033885 & 5.835872 & -2.477053 \\
\hline 0 & 0.250151 & 0.991713 & -1.023623 \\
\hline C & -0.705189 & 0.401920 & -0.530464 \\
\hline 0 & -0.585786 & -0.516632 & 0.392598 \\
\hline C & -2.148069 & 0.807571 & -0.829593 \\
\hline $\mathrm{H}$ & 0.362454 & -0.743224 & 0.477312 \\
\hline $\mathrm{N}$ & -2.994004 & -0.358099 & -0.925864 \\
\hline $\mathrm{N}$ & -2.736069 & 1.551624 & 0.262371 \\
\hline $\mathrm{H}$ & -2.107011 & 1.370958 & -1.771744 \\
\hline C & -3.980185 & -0.415040 & 0.153697 \\
\hline C & -2.561307 & -1.301029 & -1.818563 \\
\hline C & -3.423628 & 0.626558 & 1.155623 \\
\hline C & -2.168231 & 2.710236 & 0.782630 \\
\hline $\mathrm{H}$ & -4.950594 & -0.036624 & -0.208123 \\
\hline C & -4.192311 & -1.755485 & 0.805127 \\
\hline 0 & -1.457921 & -1.130543 & -2.333902 \\
\hline C & -3.405728 & -2.471262 & -2.134020 \\
\hline $\mathrm{H}$ & -2.707172 & 0.125306 & 1.828568 \\
\hline C & -4.516240 & 1.255616 & 1.973924 \\
\hline 0 & -2.041273 & 2.849077 & 1.983056 \\
\hline C & -1.799683 & 3.786262 & -0.177257 \\
\hline C & -3.125960 & -2.615558 & 1.084511 \\
\hline C & -5.481111 & -2.115608 & 1.202045 \\
\hline C & -2.759694 & -3.663960 & -2.477177 \\
\hline C & -4.803009 & -2.422920 & -2.150843 \\
\hline C & -4.752165 & 0.799348 & 3.271258 \\
\hline C & -5.356780 & 2.229431 & 1.426627 \\
\hline C & -2.369757 & 3.903593 & -1.452543 \\
\hline C & -0.943538 & 4.796659 & 0.271375 \\
\hline C & -3.353100 & -3.826894 & 1.730559 \\
\hline $\mathrm{H}$ & -2.113170 & -2.338287 & 0.785771 \\
\hline
\end{tabular}




$\begin{array}{lrrr}\text { C } & -5.707654 & -3.323981 & 1.858143 \\ \text { H } & -6.317151 & -1.442618 & 0.993969 \\ \text { C } & -3.500233 & -4.797274 & -2.783358 \\ \text { H } & -1.668083 & -3.684975 & -2.494183 \\ \text { C } & -5.536770 & -3.555971 & -2.477379 \\ \text { H } & -5.326011 & -1.490675 & -1.930471 \\ \text { C } & -5.813906 & 1.309710 & 4.015496 \\ \text { H } & -4.098355 & 0.035943 & 3.700460 \\ \text { C } & -6.412288 & 2.746313 & 2.172628 \\ \text { H } & -5.175766 & 2.593842 & 0.411759 \\ \text { C } & -2.049515 & 4.978346 & -2.271169 \\ \text { H } & -3.103409 & 3.170999 & -1.794264 \\ \text { C } & -0.638563 & 5.877655 & -0.547632 \\ \text { H } & -0.541788 & 4.729415 & 1.284403 \\ \text { C } & -4.644583 & -4.185574 & 2.117922 \\ \text { H } & -2.515758 & -4.498964 & 1.932692 \\ \text { H } & -6.721181 & -3.594727 & 2.162113 \\ \text { C } & -4.901692 & -4.767185 & -2.781490 \\ \text { H } & -2.984748 & -5.727508 & -3.035639 \\ \text { H } & -6.628125 & -3.502579 & -2.496686 \\ \text { C } & -6.644574 & 2.285567 & 3.468632 \\ \text { H } & -5.989879 & 0.946095 & 5.030344 \\ \text { H } & -7.058051 & 3.514415 & 1.740956 \\ \text { C } & -1.171627 & 5.982939 & -1.837969 \\ \text { H } & -2.507739 & 5.055557 & -3.260635 \\ \text { H } & 0.017356 & 6.669158 & -0.176310 \\ \text { H } & -4.820992 & -5.137732 & 2.623342 \\ \text { H } & -7.473355 & 2.690810 & 4.053490 \\ \text { C } & -0.840449 & 7.142320 & -2.727636 \\ \text { H } & -1.747523 & 7.708612 & -2.991088 \\ \text { H } & -0.137630 & 7.836088 & -2.246842 \\ \text { H } & -0.391208 & 6.799799 & -3.673093 \\ \text { C } & -5.700135 & -6.000436 & -3.075615 \\ \text { H } & -5.148550 & -6.695360 & -3.724161 \\ \text { H } & -5.934568 & -6.538207 & -2.141638 \\ \text { H } & -6.656372 & -5.757468 & -3.560206 \\ & & & \end{array}$

\section{TS(5-6)}

Lowest frequency $=-1325.0231 \mathrm{~cm}^{-1}$ Charge $=1$, Multiplicity $=1$

\section{1}

$\begin{array}{llrl}\mathrm{N} & 3.605951 & -1.374171 & 1.274731 \\ \mathrm{~N} & 2.181620 & 0.523382 & 1.111560 \\ \mathrm{C} & 7.509739 & -2.557249 & 1.969160 \\ \mathrm{C} & 7.181363 & -1.237052 & 2.320191 \\ \mathrm{H} & 7.950563 & -0.585530 & 2.740324 \\ \mathrm{C} & 5.892342 & -0.742967 & 2.142451 \\ \mathrm{H} & 5.640117 & 0.282507 & 2.422519 \\ \mathrm{C} & 4.940636 & -1.609132 & 1.601425 \\ \mathrm{C} & 5.249225 & -2.944756 & 1.247858 \\ \mathrm{C} & 6.556315 & -3.415503 & 1.435005 \\ \mathrm{H} & 6.820293 & -4.440348 & 1.164325 \\ \mathrm{C} & 4.040216 & -3.529937 & 0.727115 \\ \mathrm{C} & 3.075828 & -2.559579 & 0.738661 \\ \mathrm{C} & 1.695597 & -2.679322 & 0.189245 \\ \mathrm{H} & 0.966307 & -2.470300 & 0.995027\end{array}$

\begin{tabular}{|c|c|c|c|}
\hline C & 1.467814 & -4.115981 & -0.328954 \\
\hline $\mathrm{H}$ & 1.767307 & -4.148865 & -1.391319 \\
\hline $\mathrm{H}$ & 0.387524 & -4.329848 & -0.312411 \\
\hline C & 2.250755 & -5.188822 & 0.417561 \\
\hline $\mathrm{H}$ & 1.960417 & -5.194707 & 1.482191 \\
\hline $\mathrm{H}$ & 1.997847 & -6.180948 & 0.013559 \\
\hline C & 3.753604 & -4.935424 & 0.311356 \\
\hline $\mathrm{H}$ & 4.098836 & -5.124021 & -0.722067 \\
\hline $\mathrm{H}$ & 4.314132 & -5.636387 & 0.950395 \\
\hline C & 1.482059 & -1.687591 & -0.966249 \\
\hline $\mathrm{H}$ & 2.259613 & -1.886318 & -1.705792 \\
\hline $\mathrm{H}$ & 0.544520 & -1.916336 & -1.502420 \\
\hline C & 2.865775 & -0.345526 & 1.876120 \\
\hline C & 2.875007 & -0.219652 & 3.265857 \\
\hline $\mathrm{H}$ & 3.426462 & -0.955118 & 3.852817 \\
\hline C & 2.191849 & 0.825435 & 3.866615 \\
\hline $\mathrm{H}$ & 2.198150 & 0.929562 & 4.954373 \\
\hline C & 1.483173 & 1.739766 & 3.080219 \\
\hline C & 1.502473 & 1.520929 & 1.703582 \\
\hline $\mathrm{H}$ & 0.953959 & 2.182623 & 1.029787 \\
\hline C & 0.735894 & 2.895543 & 3.666154 \\
\hline $\mathrm{H}$ & -0.324548 & 2.875179 & 3.369869 \\
\hline $\mathrm{H}$ & 0.794224 & 2.887710 & 4.762227 \\
\hline $\mathrm{H}$ & 1.158013 & 3.850985 & 3.316056 \\
\hline $\mathrm{H}$ & 8.531475 & -2.911396 & 2.120806 \\
\hline Ru & 2.242583 & 0.496412 & -0.994025 \\
\hline C & 4.019631 & -0.221448 & -2.138860 \\
\hline C & 3.871385 & 1.959105 & -0.988909 \\
\hline C & 2.983137 & 0.229703 & -3.025109 \\
\hline C & 4.425548 & 0.648269 & -1.107805 \\
\hline C & 2.879979 & 2.427975 & -1.872307 \\
\hline $\mathrm{H}$ & 4.171299 & 2.576485 & -0.142053 \\
\hline C & 2.426449 & 1.511631 & -2.882676 \\
\hline $\mathrm{H}$ & 2.574021 & -0.451416 & -3.774222 \\
\hline $\mathrm{H}$ & 5.153524 & 0.298637 & -0.372802 \\
\hline $\mathrm{H}$ & 1.565434 & 1.783560 & -3.496718 \\
\hline C & 4.653122 & -1.564408 & -2.298651 \\
\hline $\mathrm{H}$ & 3.966988 & -2.297118 & -2.745204 \\
\hline $\mathrm{H}$ & 5.017030 & -1.956924 & -1.339953 \\
\hline $\mathrm{H}$ & 5.513919 & -1.467010 & -2.979779 \\
\hline C & 2.227257 & 3.785428 & -1.740652 \\
\hline C & 2.690908 & 4.557554 & -0.514632 \\
\hline C & 2.455915 & 4.601095 & -3.015012 \\
\hline $\mathrm{H}$ & 1.142371 & 3.600064 & -1.644562 \\
\hline $\mathrm{H}$ & 2.478502 & 4.020376 & 0.422158 \\
\hline $\mathrm{H}$ & 2.167701 & 5.520588 & -0.463755 \\
\hline $\mathrm{H}$ & 3.770652 & 4.772046 & -0.554923 \\
\hline $\mathrm{H}$ & 2.093398 & 4.080048 & -3.913186 \\
\hline $\mathrm{H}$ & 3.525365 & 4.820704 & -3.159312 \\
\hline $\mathrm{H}$ & 1.917979 & 5.558016 & -2.948705 \\
\hline 0 & 0.271557 & 1.228322 & -1.047497 \\
\hline C & -0.644299 & 0.543391 & -0.530448 \\
\hline 0 & -0.397371 & -0.448017 & 0.205552 \\
\hline C & -2.101725 & 0.943290 & -0.781218 \\
\hline $\mathrm{H}$ & 0.709212 & -0.781722 & -0.268931 \\
\hline $\mathrm{N}$ & -2.916744 & -0.237568 & -0.937283 \\
\hline $\mathrm{N}$ & -2.696043 & 1.602789 & 0.361544 \\
\hline $\mathrm{H}$ & -2.101454 & 1.561814 & -1.687549 \\
\hline C & -3.837146 & -0.425754 & 0.184803 \\
\hline C & -2.516160 & -1.099124 & -1.921517 \\
\hline C & -3.304540 & 0.594690 & 1.221935 \\
\hline
\end{tabular}




\begin{tabular}{|c|c|c|c|}
\hline C & -2.181265 & 2.760993 & 0.918629 \\
\hline $\mathrm{H}$ & -4.849964 & -0.100315 & -0.104002 \\
\hline C & -3.917877 & -1.815739 & 0.759614 \\
\hline 0 & -1.458573 & -0.870302 & -2.503371 \\
\hline C & -3.360669 & -2.268479 & -2.256043 \\
\hline $\mathrm{H}$ & -2.535244 & 0.102520 & 1.841688 \\
\hline C & -4.404698 & 1.109321 & 2.107516 \\
\hline 0 & -2.103141 & 2.889030 & 2.125329 \\
\hline C & -1.784350 & 3.859131 & -0.008921 \\
\hline C & -2.771303 & -2.593047 & 0.952125 \\
\hline C & -5.156187 & -2.311984 & 1.169038 \\
\hline C & -2.720883 & -3.440870 & -2.671116 \\
\hline C & -4.757539 & -2.229834 & -2.227575 \\
\hline C & -4.571298 & 0.566550 & 3.381946 \\
\hline C & -5.320644 & 2.057092 & 1.641967 \\
\hline C & -2.349246 & 4.037036 & -1.278153 \\
\hline C & -0.890046 & 4.822335 & 0.468202 \\
\hline C & -2.870731 & -3.856792 & 1.526140 \\
\hline $\mathrm{H}$ & -1.799654 & -2.199628 & 0.646645 \\
\hline C & -5.254373 & -3.574084 & 1.752311 \\
\hline $\mathrm{H}$ & -6.054090 & -1.704509 & 1.027950 \\
\hline C & -3.464419 & -4.565131 & -3.003077 \\
\hline $\mathrm{H}$ & -1.630376 & -3.456293 & -2.723186 \\
\hline C & -5.496059 & -3.351885 & -2.582410 \\
\hline $\mathrm{H}$ & -5.277373 & -1.311318 & -1.948755 \\
\hline C & -5.637764 & 0.966890 & 4.184677 \\
\hline $\mathrm{H}$ & -3.859035 & -0.177795 & 3.746855 \\
\hline C & -6.381026 & 2.464389 & 2.446383 \\
\hline $\mathrm{H}$ & -5.194478 & 2.487766 & 0.644871 \\
\hline C & -1.985595 & 5.122505 & -2.065710 \\
\hline H & -3.108931 & 3.342622 & -1.642220 \\
\hline C & -0.538831 & 5.912241 & -0.319315 \\
\hline $\mathrm{H}$ & -0.495062 & 4.712245 & 1.479796 \\
\hline C & -4.112316 & -4.352289 & 1.926169 \\
\hline $\mathrm{H}$ & -1.970986 & -4.461831 & 1.663793 \\
\hline $\mathrm{H}$ & -6.229649 & -3.952195 & 2.066787 \\
\hline C & -4.865254 & -4.544480 & -2.958145 \\
\hline $\mathrm{H}$ & -2.951955 & -5.480511 & -3.310223 \\
\hline $\mathrm{H}$ & -6.587817 & -3.304142 & -2.566636 \\
\hline C & -6.543262 & 1.918222 & 3.719750 \\
\hline $\mathrm{H}$ & -5.758915 & 0.535965 & 5.181083 \\
\hline $\mathrm{H}$ & -7.085790 & 3.213666 & 2.078542 \\
\hline C & -1.067455 & 6.076884 & -1.605810 \\
\hline $\mathrm{H}$ & -2.439455 & 5.246003 & -3.052490 \\
\hline $\mathrm{H}$ & 0.148690 & 6.664418 & 0.076334 \\
\hline $\mathrm{H}$ & -4.188559 & -5.345290 & 2.375006 \\
\hline $\mathrm{H}$ & -7.375969 & 2.237333 & 4.350594 \\
\hline C & -0.705551 & 7.262365 & -2.448749 \\
\hline $\mathrm{H}$ & 0.225156 & 7.736111 & -2.106564 \\
\hline $\mathrm{H}$ & -0.586788 & 6.984263 & -3.506312 \\
\hline $\mathrm{H}$ & -1.498601 & 8.026947 & -2.403850 \\
\hline C & -5.665362 & -5.769724 & -3.281150 \\
\hline $\mathrm{H}$ & -5.140768 & -6.419566 & -3.995714 \\
\hline $\mathrm{H}$ & -5.845623 & -6.363070 & -2.369041 \\
\hline $\mathrm{H}$ & -6.647561 & -5.512577 & -3.702263 \\
\hline
\end{tabular}

\section{I-6 ${ }^{S}$}

Lowest frequency $=7.8345 \mathrm{~cm}^{-1}$ Charge $=1$, Multiplicity $=1$

131

\begin{tabular}{|c|c|c|c|}
\hline$N$ & 3.643060 & -1.375950 & 1.231776 \\
\hline $\mathrm{N}$ & 2.256291 & 0.551652 & 1.131924 \\
\hline C & 7.684409 & -2.135419 & 1.662160 \\
\hline C & 7.246911 & -0.843561 & 2.001715 \\
\hline $\mathrm{H}$ & 7.972659 & -0.105253 & 2.349461 \\
\hline C & 5.904350 & -0.489100 & 1.907413 \\
\hline $\mathrm{H}$ & 5.563273 & 0.511804 & 2.182881 \\
\hline C & 5.013917 & -1.465324 & 1.457238 \\
\hline C & 5.429907 & -2.777743 & 1.128438 \\
\hline C & 6.789364 & -3.106294 & 1.229756 \\
\hline $\mathrm{H}$ & 7.138118 & -4.109889 & 0.975888 \\
\hline C & 4.248673 & -3.504218 & 0.737717 \\
\hline C & 3.191991 & -2.635911 & 0.802161 \\
\hline C & 1.789549 & -2.922321 & 0.376794 \\
\hline $\mathrm{H}$ & 1.099793 & -2.736712 & 1.220232 \\
\hline C & 1.672045 & -4.391542 & -0.079653 \\
\hline $\mathrm{H}$ & 1.885108 & -4.436524 & -1.162585 \\
\hline $\mathrm{H}$ & 0.626338 & -4.714947 & 0.037818 \\
\hline C & 2.622483 & -5.347488 & 0.630100 \\
\hline $\mathrm{H}$ & 2.419064 & -5.342106 & 1.714510 \\
\hline $\mathrm{H}$ & 2.443487 & -6.374741 & 0.278472 \\
\hline C & 4.077226 & -4.947155 & 0.392516 \\
\hline $\mathrm{H}$ & 4.358716 & -5.141852 & -0.658932 \\
\hline $\mathrm{H}$ & 4.755794 & -5.561525 & 1.005638 \\
\hline C & 1.371865 & -2.020460 & -0.779969 \\
\hline $\mathrm{H}$ & 2.073033 & -2.106591 & -1.613462 \\
\hline $\mathrm{H}$ & 0.363094 & -2.232764 & -1.159314 \\
\hline C & 2.865539 & -0.390160 & 1.871328 \\
\hline C & 2.772780 & -0.378284 & 3.259789 \\
\hline $\mathrm{H}$ & 3.278451 & -1.163380 & 3.823528 \\
\hline C & 2.047941 & 0.623931 & 3.889844 \\
\hline $\mathrm{H}$ & 1.966137 & 0.637630 & 4.979407 \\
\hline C & 1.415740 & 1.610545 & 3.128778 \\
\hline C & 1.549008 & 1.512043 & 1.743681 \\
\hline $\mathrm{H}$ & 1.048501 & 2.224198 & 1.084816 \\
\hline C & 0.607055 & 2.710149 & 3.739409 \\
\hline $\mathrm{H}$ & -0.440954 & 2.664829 & 3.400213 \\
\hline $\mathrm{H}$ & 0.621232 & 2.651094 & 4.835282 \\
\hline $\mathrm{H}$ & 1.002277 & 3.696068 & 3.449357 \\
\hline $\mathrm{H}$ & 8.745589 & -2.378345 & 1.747025 \\
\hline Ru & 2.272254 & 0.492879 & -0.973158 \\
\hline C & 3.904319 & -0.304054 & -2.275060 \\
\hline C & 3.925712 & 1.840170 & -1.079989 \\
\hline C & 2.813049 & 0.195412 & -3.066692 \\
\hline C & 4.457967 & 0.537842 & -1.291254 \\
\hline C & 2.860142 & 2.357145 & -1.864914 \\
\hline $\mathrm{H}$ & 4.298708 & 2.425417 & -0.238988 \\
\hline C & 2.319690 & 1.493847 & -2.876852 \\
\hline $\mathrm{H}$ & 2.305497 & -0.471182 & -3.767224 \\
\hline $\mathrm{H}$ & 5.238603 & 0.156126 & -0.630255 \\
\hline $\mathrm{H}$ & 1.414705 & 1.799372 & -3.405595 \\
\hline C & 4.441479 & -1.682424 & -2.482521 \\
\hline $\mathrm{H}$ & 3.704540 & -2.346919 & -2.952832 \\
\hline $\mathrm{H}$ & 4.780629 & -2.130668 & -1.53872 \\
\hline $\mathrm{H}$ & 5.307057 & -1.619892 & -3.161919 \\
\hline C & 2.251900 & 3.721740 & -1.639698 \\
\hline C & 2.797751 & 4.432098 & -0.410097 \\
\hline
\end{tabular}




\begin{tabular}{|c|c|c|c|}
\hline C & 2.448801 & 4.576971 & -2.893716 \\
\hline $\mathrm{H}$ & 1.168035 & 3.559866 & -1.499928 \\
\hline $\mathrm{H}$ & 2.642626 & 3.853442 & 0.512671 \\
\hline $\mathrm{H}$ & 2.281515 & 5.391762 & -0.281217 \\
\hline $\mathrm{H}$ & 3.873663 & 4.647253 & -0.508794 \\
\hline H & 2.010641 & 4.110575 & -3.788188 \\
\hline $\mathrm{H}$ & 3.517875 & 4.753217 & -3.090869 \\
\hline $\mathrm{H}$ & 1.962539 & 5.553136 & -2.758850 \\
\hline 0 & 0.333016 & 1.129721 & -0.896841 \\
\hline C & -0.613193 & 0.522970 & -0.261057 \\
\hline 0 & -0.472092 & -0.263292 & 0.662662 \\
\hline C & -2.029187 & 0.913191 & -0.733748 \\
\hline H & 1.214793 & -0.986201 & -0.360503 \\
\hline N & -2.841796 & -0.264542 & -0.922324 \\
\hline N & -2.747231 & 1.628071 & 0.307849 \\
\hline H & -1.935635 & 1.483784 & -1.666235 \\
\hline C & -3.863527 & -0.420561 & 0.116816 \\
\hline C & -2.403873 & -1.199919 & -1.812072 \\
\hline C & -3.457948 & 0.663672 & 1.141815 \\
\hline C & -2.233221 & 2.761613 & 0.910613 \\
\hline H & -4.853237 & -0.153466 & -0.286546 \\
\hline C & -3.939076 & -1.799369 & 0.724325 \\
\hline 0 & -1.291542 & -1.097314 & -2.318246 \\
\hline C & -3.338738 & -2.307409 & -2.142760 \\
\hline H & -2.766007 & 0.222225 & 1.877527 \\
\hline C & -4.655374 & 1.226962 & 1.856656 \\
\hline 0 & -2.265744 & 2.900962 & 2.118943 \\
\hline C & -1.698716 & 3.839308 & 0.025667 \\
\hline C & -2.788623 & -2.422763 & 1.222205 \\
\hline C & -5.163205 & -2.463278 & 0.801459 \\
\hline C & -2.875826 & -3.625400 & -2.139999 \\
\hline C & -4.666416 & -2.051110 & -2.492419 \\
\hline C & -4.995042 & 0.734588 & 3.117313 \\
\hline C & -5.483839 & 2.172538 & 1.245968 \\
\hline C & -2.107773 & 4.020812 & -1.300587 \\
\hline C & -0.851676 & 4.791702 & 0.602237 \\
\hline C & -2.866985 & -3.699750 & 1.769777 \\
\hline $\mathrm{H}$ & -1.831734 & -1.897038 & 1.164401 \\
\hline C & -5.241096 & -3.741607 & 1.352197 \\
\hline H & -6.063333 & -1.980754 & 0.412856 \\
\hline C & -3.742771 & -4.669964 & -2.431769 \\
\hline H & -1.833350 & -3.826002 & -1.884062 \\
\hline C & -5.521340 & -3.100011 & -2.813221 \\
\hline H & -5.031896 & -1.021810 & -2.525379 \\
\hline C & -6.146450 & 1.181736 & 3.762406 \\
\hline $\mathrm{H}$ & -4.349400 & -0.005800 & 3.596466 \\
\hline C & -6.629333 & 2.627092 & 1.893500 \\
\hline H & -5.220544 & 2.563264 & 0.259402 \\
\hline C & -1.649340 & 5.107031 & -2.037997 \\
\hline $\mathrm{H}$ & -2.824209 & 3.331250 & -1.750480 \\
\hline C & -0.404343 & 5.880794 & -0.135705 \\
\hline $\mathrm{H}$ & -0.581385 & 4.677101 & 1.653581 \\
\hline C & -4.092104 & -4.365254 & 1.832616 \\
\hline $\mathrm{H}$ & -1.963989 & -4.181682 & 2.153817 \\
\hline H & -6.204890 & -4.253380 & 1.401236 \\
\hline C & -5.082127 & -4.428045 & -2.768057 \\
\hline $\mathrm{H}$ & -3.376404 & -5.699460 & -2.400760 \\
\hline $\mathrm{H}$ & -6.554554 & -2.885636 & -3.098519 \\
\hline C & -6.964752 & 2.130826 & 3.153204 \\
\hline H & -6.402575 & 0.790214 & 4.749547 \\
\hline $\mathrm{H}$ & -7.265160 & 3.374390 & 1.412974 \\
\hline
\end{tabular}

$\begin{array}{lrrr}\mathrm{C} & -0.789993 & 6.057820 & -1.471243 \\ \mathrm{H} & -1.988217 & 5.237443 & -3.069170 \\ \mathrm{H} & 0.239564 & 6.626289 & 0.339029 \\ \mathrm{H} & -4.151352 & -5.369095 & 2.259672 \\ \mathrm{H} & -7.863917 & 2.487086 & 3.661020 \\ \mathrm{C} & -0.349742 & 7.260383 & -2.251029 \\ \mathrm{H} & -1.096587 & 8.067445 & -2.168367 \\ \mathrm{H} & 0.602614 & 7.663083 & -1.877322 \\ \mathrm{H} & -0.238094 & 7.033194 & -3.321023 \\ \mathrm{C} & -6.019513 & -5.564459 & -3.045996 \\ \mathrm{H} & -5.521429 & -6.372619 & -3.601174 \\ \mathrm{H} & -6.389908 & -5.998813 & -2.102391 \\ \mathrm{H} & -6.894594 & -5.236827 & -3.624139\end{array}$

\section{$\mathrm{RCO}_{2} \mathrm{H}$}

Lowest frequency $=16.7687 \mathrm{~cm}^{-1}$

Charge $=0$, Multiplicity $=1$

66

$\begin{array}{lrrr}\text { O } & 2.201352 & -2.283298 & 1.872439 \\ \mathrm{C} & 1.255299 & -1.594178 & 1.619268 \\ \mathrm{O} & 0.390227 & -1.155771 & 2.544166 \\ \mathrm{C} & 0.982049 & -1.003391 & 0.228907 \\ \mathrm{H} & 0.675668 & -1.536764 & 3.389165 \\ \mathrm{~N} & -0.423005 & -0.865346 & -0.074472 \\ \mathrm{~N} & 1.464372 & 0.366229 & 0.138437 \\ \mathrm{H} & 1.486694 & -1.679753 & -0.475045 \\ \mathrm{C} & -0.837679 & 0.527917 & -0.183858 \\ \mathrm{C} & -1.153488 & -2.023009 & -0.063874 \\ \mathrm{C} & 0.370447 & 1.278718 & 0.426706 \\ \mathrm{C} & 2.754154 & 0.731545 & 0.508849 \\ \mathrm{H} & -0.899923 & 0.823894 & -1.245058 \\ \mathrm{C} & -2.128278 & 0.900735 & 0.497172 \\ \mathrm{O} & -0.624622 & -3.053663 & 0.325150 \\ \mathrm{C} & -2.566825 & -1.998659 & -0.529315 \\ \mathrm{H} & 0.220725 & 1.375363 & 1.516114 \\ \mathrm{C} & 0.522765 & 2.648679 & -0.175347 \\ \mathrm{O} & 2.957553 & 1.745735 & 1.141594 \\ \mathrm{C} & 3.856354 & -0.153497 & 0.041351 \\ \mathrm{C} & -2.479986 & 0.363862 & 1.738921 \\ \mathrm{C} & -2.958558 & 1.854026 & -0.094822 \\ \mathrm{C} & -3.471679 & -2.852484 & 0.108375 \\ \mathrm{C} & -3.008177 & -1.226483 & -1.606235 \\ \mathrm{C} & 0.004043 & 3.757129 & 0.495025 \\ \mathrm{C} & 1.089069 & 2.821232 & -1.441433 \\ \mathrm{C} & 3.820369 & -0.801886 & -1.196095 \\ \mathrm{C} & 5.000414 & -0.267115 & 0.835882 \\ \mathrm{C} & -3.656739 & 0.763576 & 2.365695 \\ \mathrm{H} & -1.825289 & -0.372460 & 2.209965 \\ \mathrm{C} & -4.132103 & 2.260788 & 0.536904 \\ \mathrm{H} & -2.682084 & 2.280665 & -1.062714 \\ \mathrm{C} & -4.801121 & -2.891644 & -0.290738 \\ \mathrm{H} & -3.109505 & -3.482778 & 0.922914 \\ \mathrm{C} & -4.336006 & -1.285285 & -2.014516 \\ \mathrm{H} & -2.309349 & -0.586481 & -2.147793 \\ \mathrm{C} & 0.050577 & 5.021520 & -0.088951 \\ \mathrm{H} & -0.439004 & 3.625478 & 1.485281\end{array}$




$\begin{array}{lrrrrrrr}\mathrm{C} & 1.143890 & 4.084887 & -2.022658 & \mathrm{H} & 1.600394 & 4.210164 & -3.007471 \\ \mathrm{H} & 1.502755 & 1.956530 & -1.966863 & \mathrm{C} & 6.037775 & -1.716666 & -0.814619 \\ \mathrm{C} & 4.901570 & -1.568264 & -1.618290 & \mathrm{H} & 4.865691 & -2.060070 & -2.593993 \\ \mathrm{H} & 2.950162 & -0.683471 & -1.846394 & \mathrm{H} & 6.953168 & -1.138547 & 1.053553 \\ \mathrm{C} & 6.069390 & -1.046991 & 0.416321 & \mathrm{H} & -5.411252 & 2.021356 & 2.260399 \\ \mathrm{H} & 5.032840 & 0.267549 & 1.787135 & \mathrm{H} & 0.665932 & 6.181138 & -1.804323 \\ \mathrm{C} & -4.487055 & 1.711439 & 1.766857 & \mathrm{C} & 7.182579 & -2.584271 & -1.245910 \\ \mathrm{H} & -3.929500 & 0.330255 & 3.330922 & \mathrm{H} & 8.148607 & -2.163650 & -0.930239 \\ \mathrm{H} & -4.774211 & 3.006153 & 0.061818 & \mathrm{H} & 7.100852 & -3.587111 & -0.794246 \\ \mathrm{C} & -5.260037 & -2.102743 & -1.353910 & \mathrm{H} & 7.202816 & -2.715808 & -2.337037 \\ \mathrm{H} & -5.500996 & -3.552286 & 0.228163 & \mathrm{C} & -6.704488 & -2.116956 & -1.757085 \\ \mathrm{H} & -4.664456 & -0.680892 & -2.864188 & \mathrm{H} & -7.166006 & -3.097883 & -1.573041 \\ \mathrm{C} & 0.621909 & 5.189077 & -1.348679 & \mathrm{H} & -7.276001 & -1.372509 & -1.177499 \\ \mathrm{H} & -0.356562 & 5.881825 & 0.447492 & \mathrm{H} & -6.830431 & -1.869766 & -2.820949\end{array}$




\section{References}

1. (a) Wunder, A.; Rothemund, M.; Schobert, R., Synthesis and anticancer activity of the proposed structure of caldoramide, an $N$-peptidyltetramate from the cyanobacterium Caldora penicillata. Tetrahedron 2018, 74, 5138-5142; (b) Jang, Y.-S.; Dieckmann, M.; Cramer, N., Cooperative Effects between Chiral $\mathrm{Cp}^{\mathrm{x}}$-Iridium(III) Catalysts and Chiral Carboxylic Acids in Enantioselective C-H Amidations of Phosphine Oxides. Angew. Chem. Int. Ed. 2017, 56, 1508815092.

2. Pesciaioli, F.; Dhawa, U.; Oliveira, J. C. A.; Yin, R.; John, M.; Ackermann, L., Enantioselective Cobalt(III)-Catalyzed $\mathrm{C}-\mathrm{H}$ Activation Enabled by Chiral Carboxylic Acid Cooperation. Angew. Chem. Int. Ed. 2018, 57, 15425-15429.

3. Halland, N.; Hansen, T.; Jørgensen, K. A., Organocatalytic Asymmetric Michael Reaction of Cyclic 1,3-Dicarbonyl Compounds and $\alpha, \beta$-Unsaturated Ketones-A Highly Atom-Economic Catalytic One-Step Formation of Optically Active Warfarin Anticoagulant. Angew. Chem. Int. Ed. 2003, 42, 4955-4957.

4. (a) Nandi, R. K.; Guillot, R.; Kouklovsky, C.; Vincent, G., Synthesis of 3,3-Spiroindolines via $\mathrm{FeCl}_{3}$-Mediated Cyclization of Aryl- or Alkene-Containing 3-Substituted N-Ac Indoles. Org. Lett. 2016, 18, 1716-1719; (b) Whyte, A.; Torelli, A.; Mirabi, B.; Prieto, L.; Rodríguez, J. F.; Lautens, M., Cobalt-Catalyzed Enantioselective Hydroarylation of 1,6-Enynes. J. Am. Chem. Soc. 2020, 142, 9510-9517.

5. Popescu, M. V.; Mekereeya, A.; Alegre-Requena, J. V.; Paton, R. S.; Smith, M. D., VisibleLight-Mediated Heterocycle Functionalization via Geometrically Interrupted [2+2] Cycloaddition. Angew. Chem. Int. Ed. 2020, 59, 23020-23024.

6. Frisch, M. J.; Trucks, G. W.; Schlegel, H. B.; Scuseria, G. E.; Robb, M. A.; Cheeseman, J. R.; Scalmani, G.; Barone, V.; Petersson, G. A.; Nakatsuji, H.; Li, X.; Caricato, M.; Marenich, A. V.; Bloino, J.; Janesko, B. G.; Gomperts, R.; Mennucci, B.; Hratchian, H. P.; Ortiz, J. V.; Izmaylov, A. F.; Sonnenberg, J. L.; Williams; Ding, F.; Lipparini, F.; Egidi, F.; Goings, J.; Peng, B.; Petrone, A.; Henderson, T.; Ranasinghe, D.; Zakrzewski, V. G.; Gao, J.; Rega, N.; Zheng, G.; Liang, W.; Hada, M.; Ehara, M.; Toyota, K.; Fukuda, R.; Hasegawa, J.; Ishida, M.; Nakajima, T.; Honda, Y.; Kitao, O.; Nakai, H.; Vreven, T.; Throssell, K.; Montgomery Jr., J. A.; Peralta, J. E.; Ogliaro, F.; Bearpark, M. J.; Heyd, J. J.; Brothers, E. N.; Kudin, K. N.; Staroverov, V. N.; Keith, T. A.; Kobayashi, R.; Normand, J.; Raghavachari, K.; Rendell, A. P.; Burant, J. C.; Iyengar, S. S.; Tomasi, 
J.; Cossi, M.; Millam, J. M.; Klene, M.; Adamo, C.; Cammi, R.; Ochterski, J. W.; Martin, R. L.; Morokuma, K.; Farkas, O.; Foresman, J. B.; Fox, D. J. Gaussian 16 Rev. A.03, Wallingford, CT, 2016.

7. Adamo, C.; Barone, V., Toward reliable density functional methods without adjustable parameters: The PBE0 model. J. Chem. Phys. 1999, 110, 6158-6170.

8. (a) Grimme, S.; Ehrlich, S.; Goerigk, L., Effect of the damping function in dispersion corrected density functional theory. J. Comput. Chem. 2011, 32, 1456-1465; (b) Grimme, S.; Antony, J.; Ehrlich, S.; Krieg, H., A consistent and accurate ab initio parametrization of density functional dispersion correction (DFT-D) for the 94 elements H-Pu. J. Chem. Phys. 2010, 132, 154104.

9. (a) Weigend, F., Accurate Coulomb-fitting basis sets for $\mathrm{H}$ to Rn. Phys. Chem. Chem. Phys. 2006, 8, 1057-1065; (b) Weigend, F.; Ahlrichs, R., Balanced basis sets of split valence, triple zeta valence and quadruple zeta valence quality for $\mathrm{H}$ to Rn: Design and assessment of accuracy. Phys. Chem. Chem. Phys. 2005, 7, 3297-3305.

10. (a) Martin, J. M. L.; Sundermann, A., Correlation consistent valence basis sets for use with the Stuttgart-Dresden-Bonn relativistic effective core potentials: The atoms Ga-Kr and In-Xe. J. Chem. Phys. 2001, 114, 3408-3420; (b) Andrae, D.; Häußermann, U.; Dolg, M.; Stoll, H.; Preuß, H., Energy-adjusted ab initio pseudopotentials for the second and third row transition elements. Theor. Chim. Acta 1990, 77, 123-141; (c) Dolg, M.; Wedig, U.; Stoll, H.; Preuss, H., Energyadjusted $a b$ initio pseudopotentials for the first row transition elements. J. Chem. Phys. 1987, 86, 866-872.

11. Zhao, Y.; Truhlar, D. G., Design of Density Functionals That Are Broadly Accurate for Thermochemistry, Thermochemical Kinetics, and Nonbonded Interactions. J. Phys. Chem. A 2005, 109, 5656-5667.

12. (a) Caldeweyher, E.; Ehlert, S.; Hansen, A.; Neugebauer, H.; Spicher, S.; Bannwarth, C.; Grimme, S., A generally applicable atomic-charge dependent London dispersion correction. J. Chem. Phys. 2019, 150, 154122; (b) Caldeweyher, E.; Bannwarth, C.; Grimme, S., Extension of the D3 dispersion coefficient model. J. Chem. Phys. 2017, 147, 034112; (c) https://www.chemie.uni-bonn.de/pctc/mulliken-center/software/dftd4. 
13. Marenich, A. V.; Cramer, C. J.; Truhlar, D. G., Universal Solvation Model Based on Solute Electron Density and on a Continuum Model of the Solvent Defined by the Bulk Dielectric Constant and Atomic Surface Tensions. J. Phys. Chem. B 2009, 113, 6378-6396.

14. (a) Contreras-García, J.; Johnson, E. R.; Keinan, S.; Chaudret, R.; Piquemal, J.-P.; Beratan, D. N.; Yang, W., NCIPLOT: A Program for Plotting Noncovalent Interaction Regions. J. Chem. Theory Comput. 2011, 7, 625-632; (b) Johnson, E. R.; Keinan, S.; Mori-Sánchez, P.; ContrerasGarcía, J.; Cohen, A. J.; Yang, W., Revealing Noncovalent Interactions. J. Am. Chem. Soc. 2010, 132, 6498-6506. 

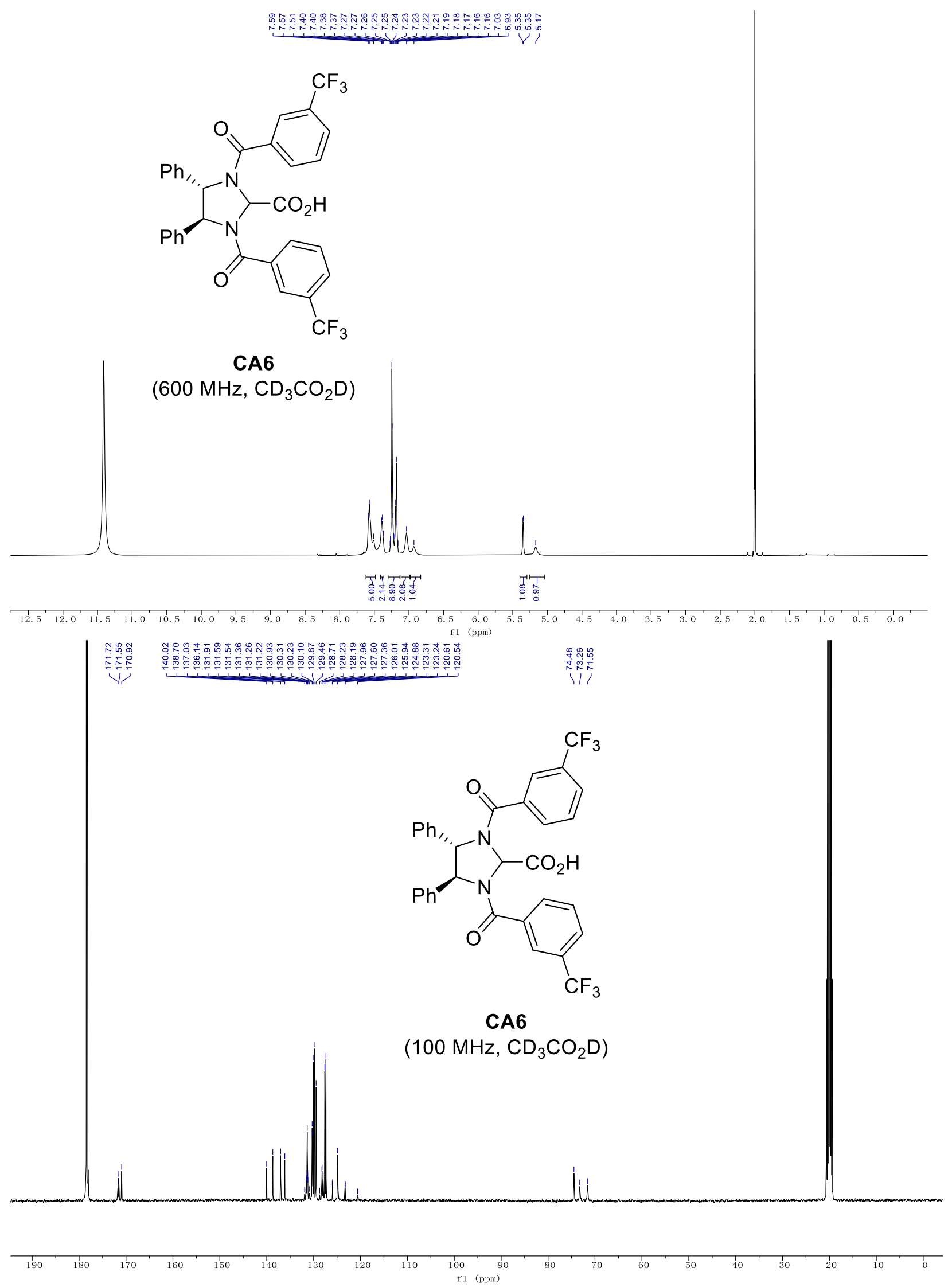


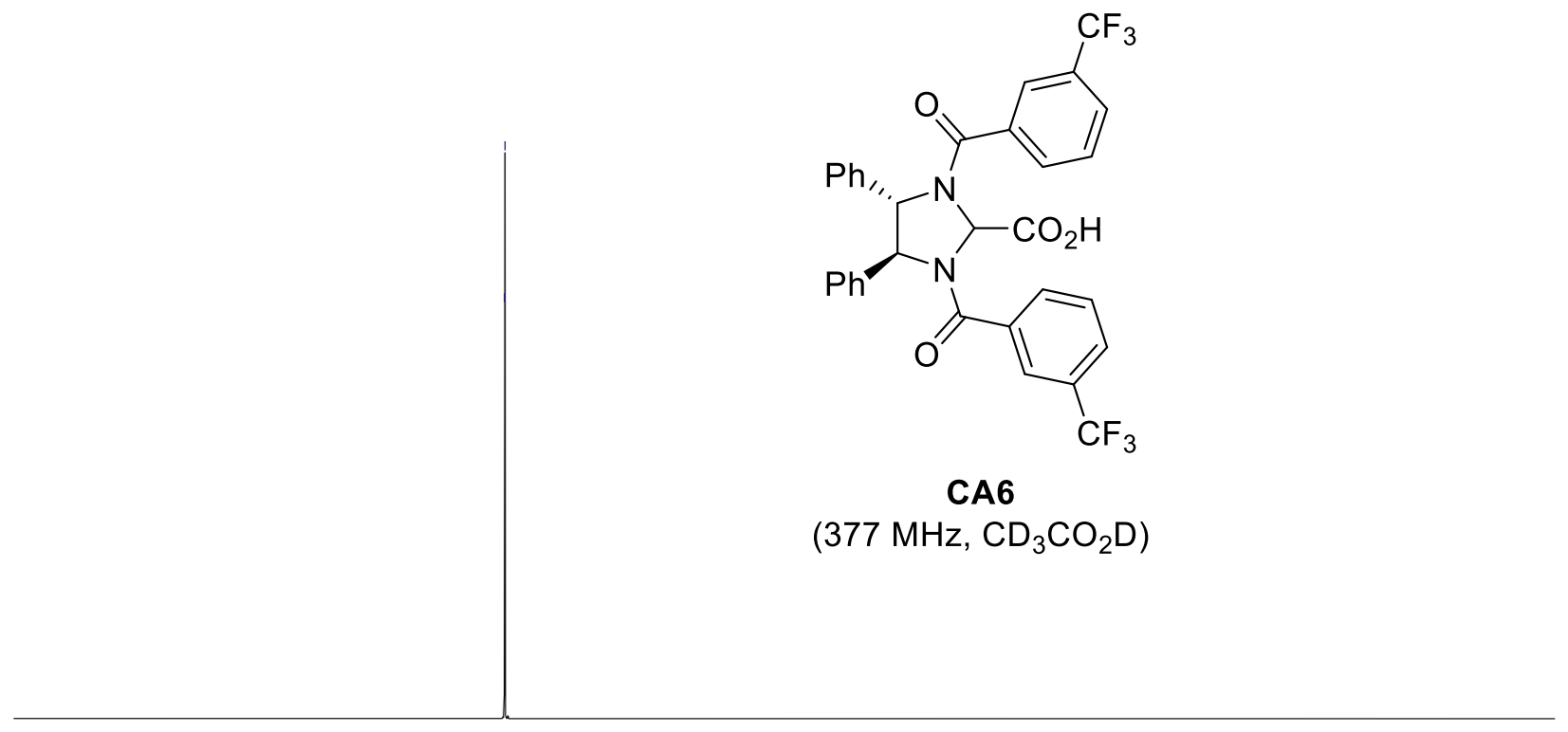

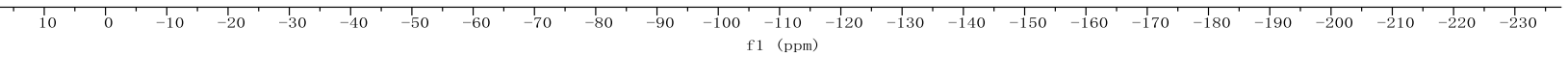



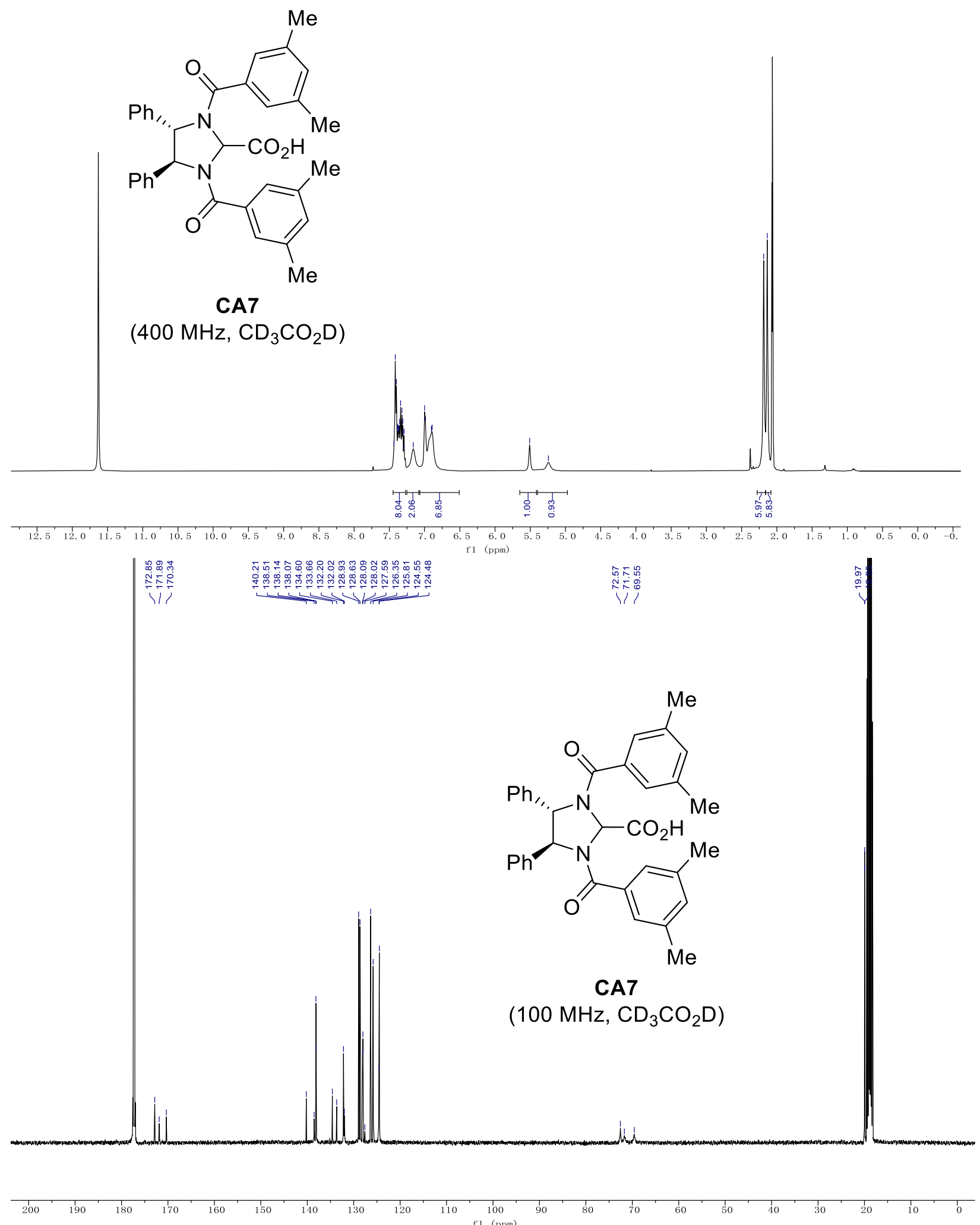


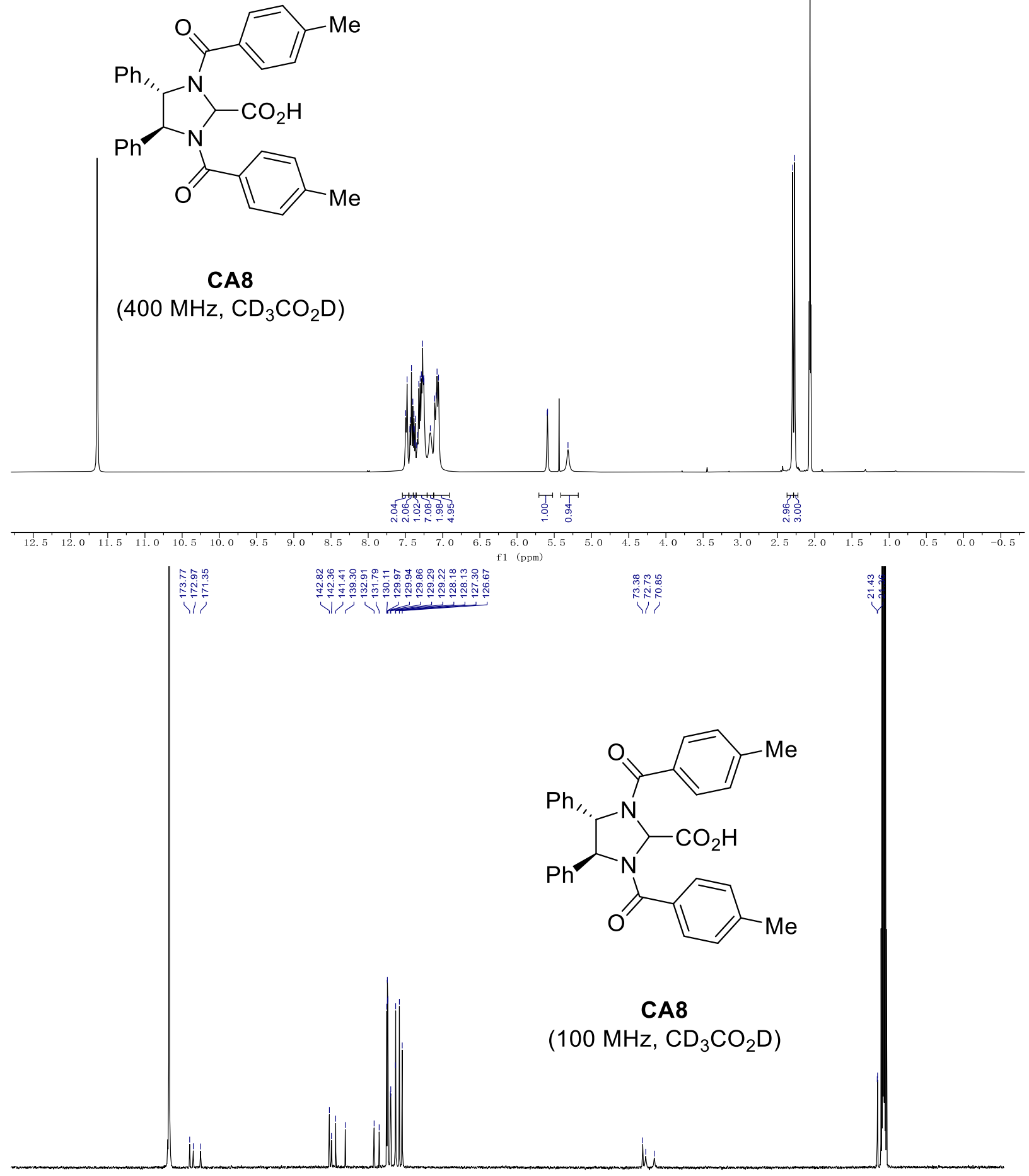

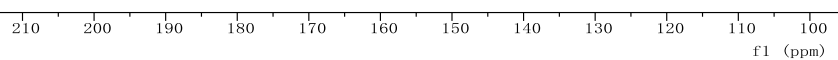




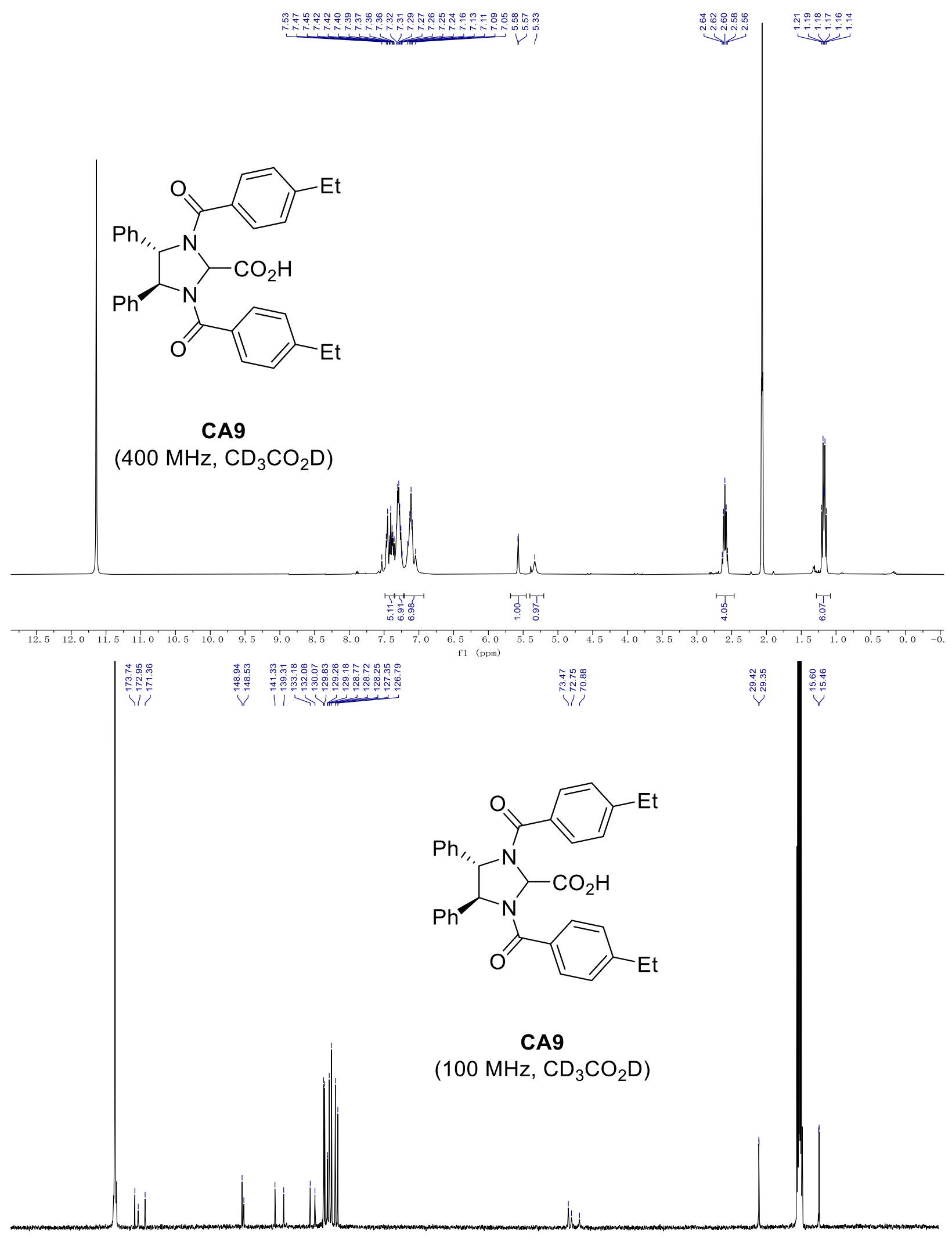

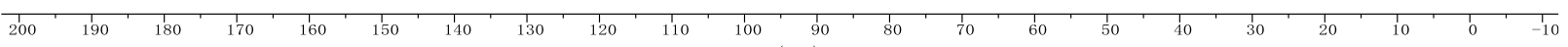



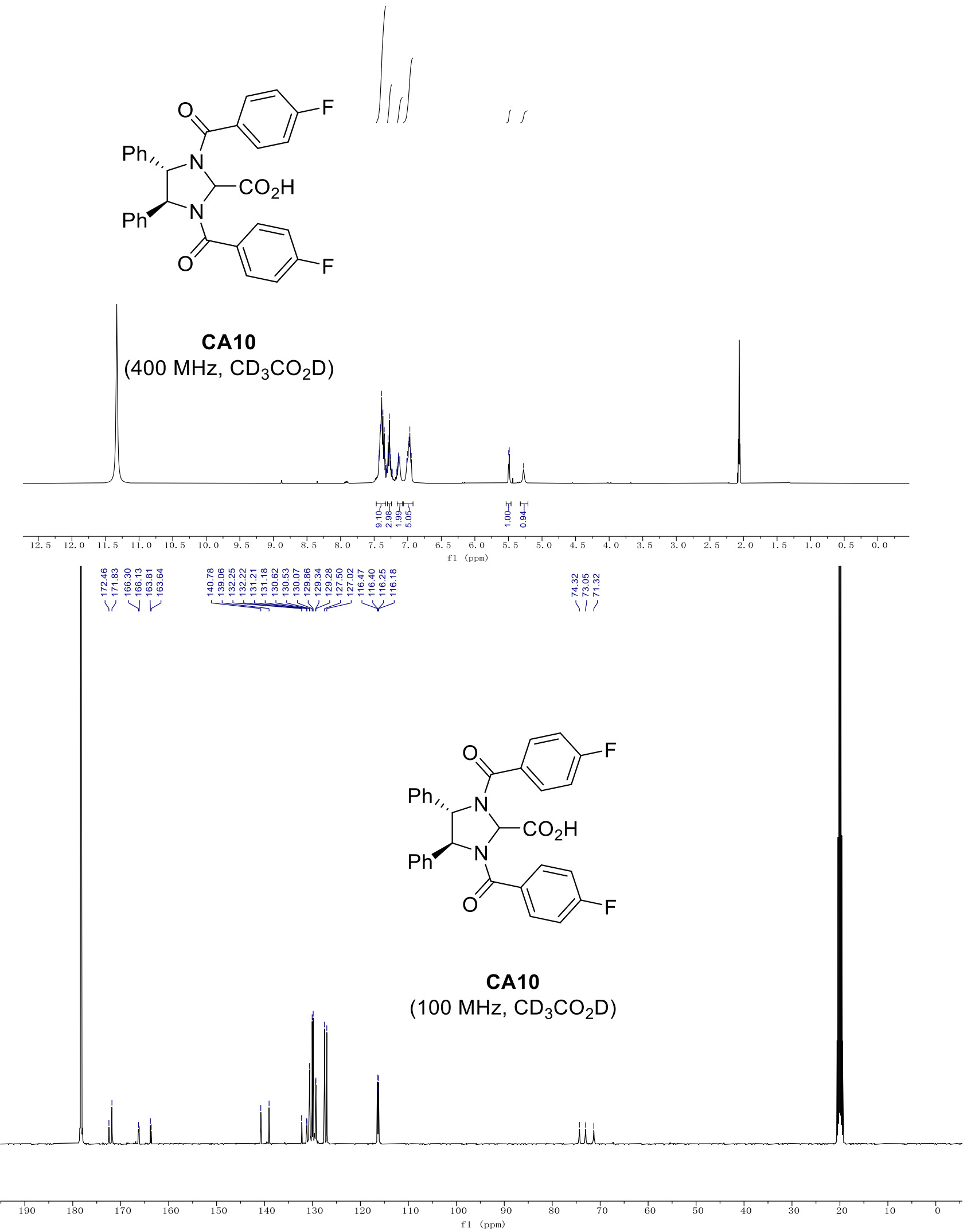


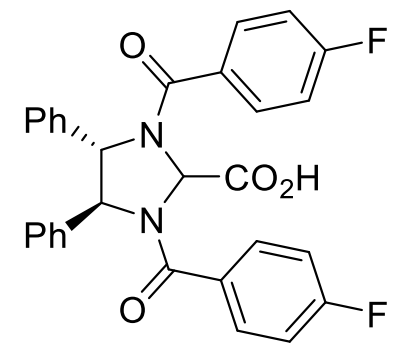

CA10

(377 MHz, $\mathrm{CD}_{3} \mathrm{CO}_{2} \mathrm{D}$ )

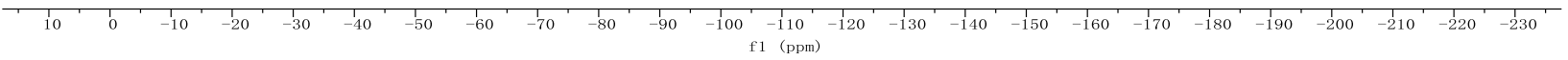




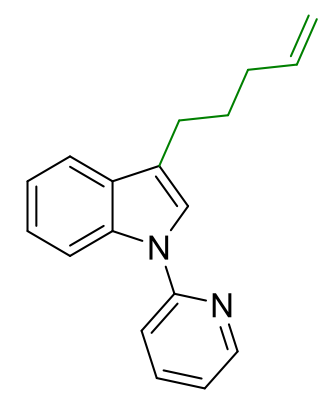

1a'
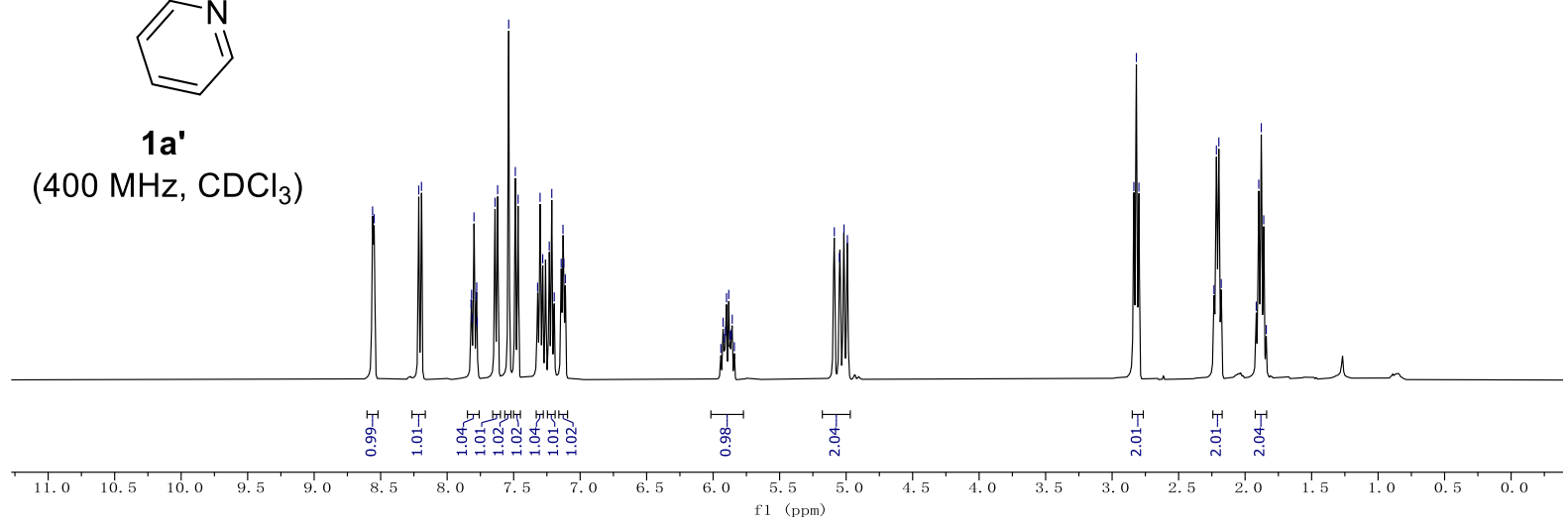

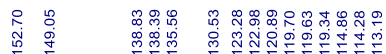

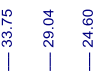

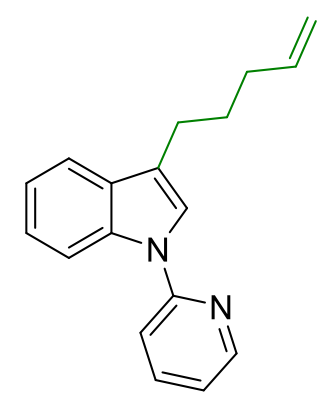

1a'

$\left(100 \mathrm{MHz} \mathrm{CDCl}_{3}\right)$

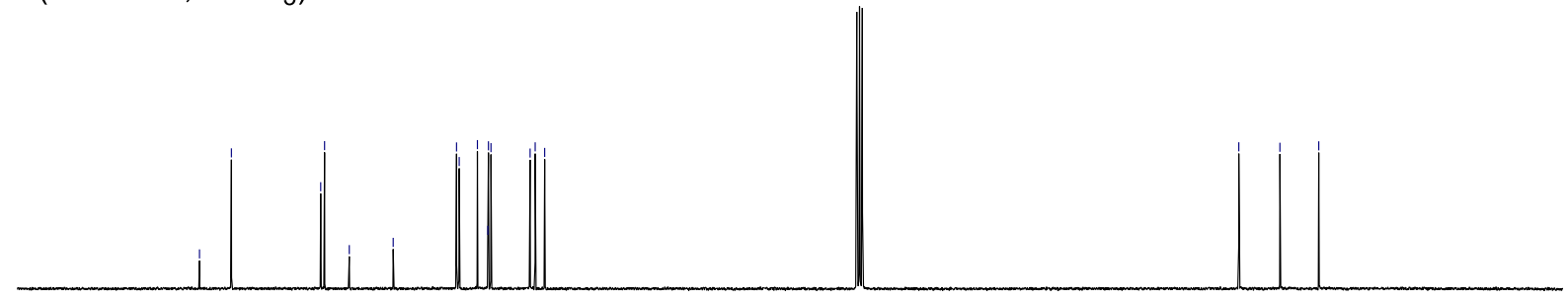

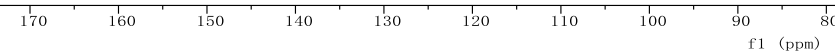




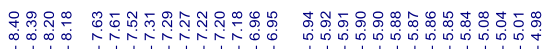

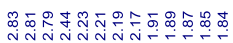

$Y \underbrace{\circ} \underbrace{2}$

$\int_{0}^{0}$
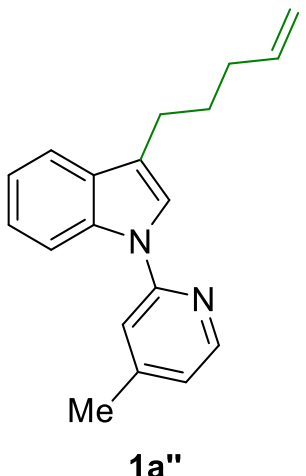

$\left(400 \mathrm{MHz}, \mathrm{CDCl}_{3}\right)$
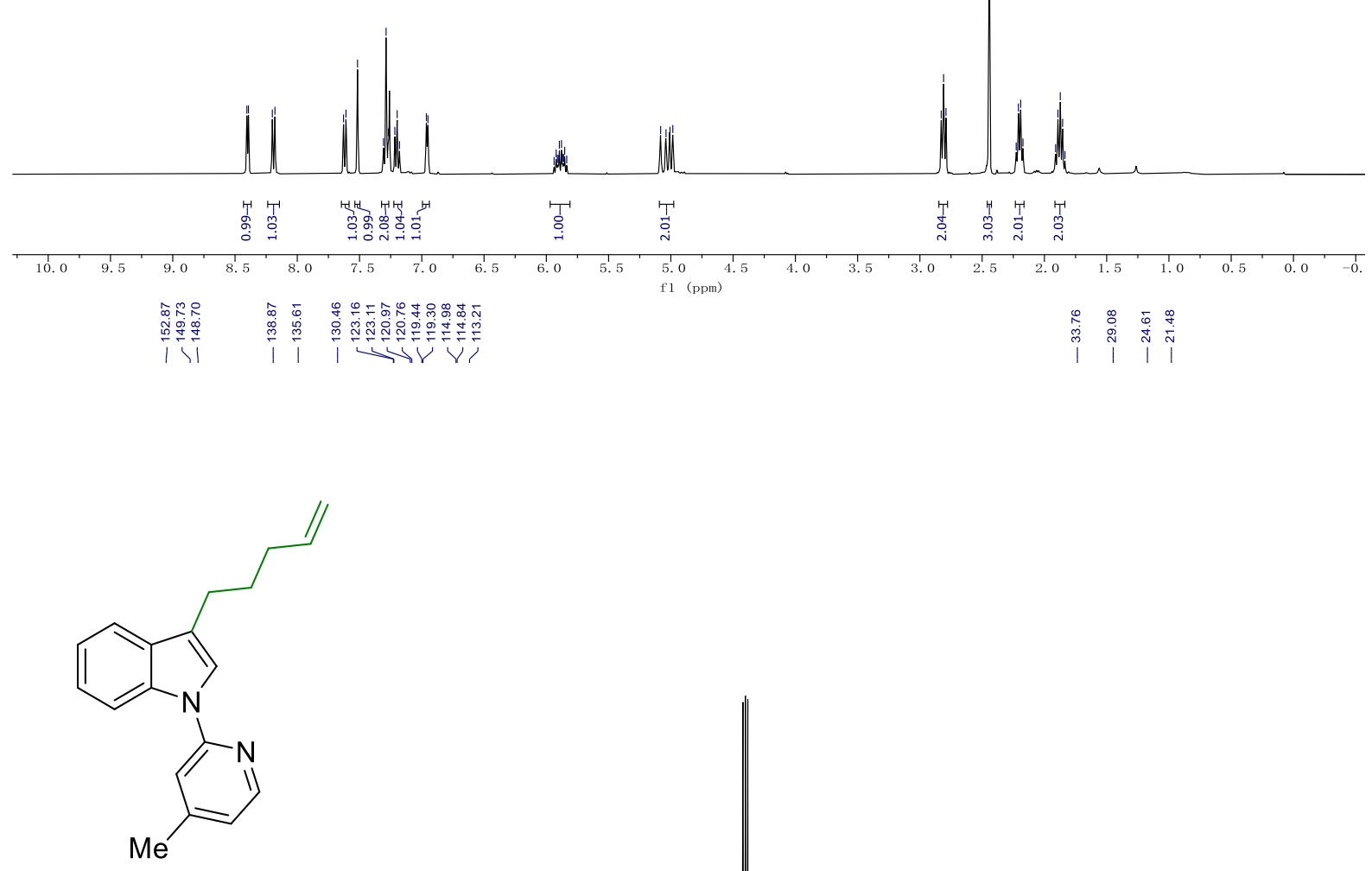

$1 a^{\prime \prime}$

(100 MHz, $\mathrm{CDCl}_{3}$ )
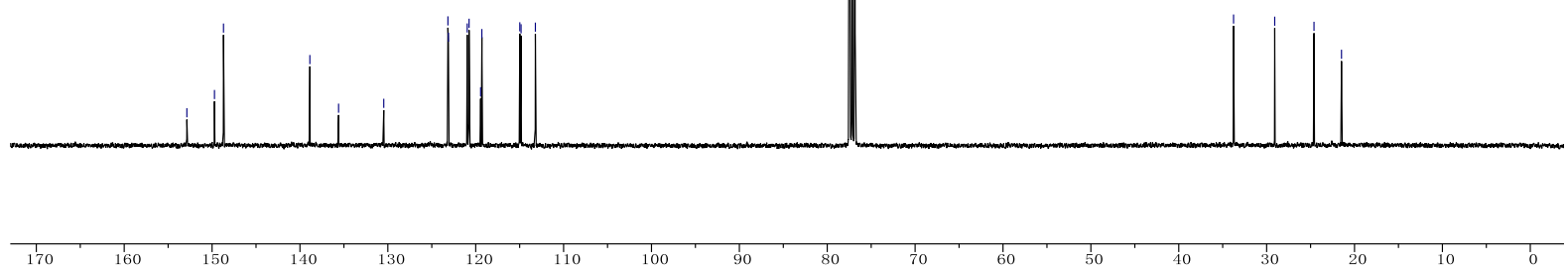

f1 80 


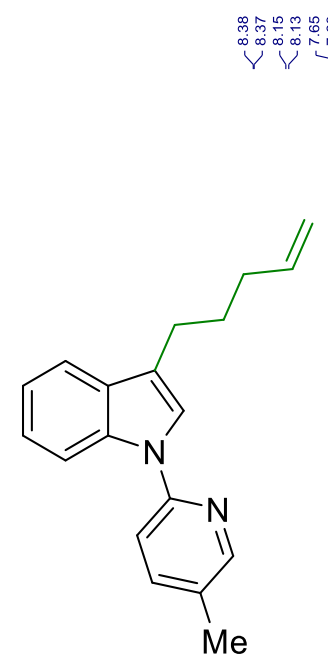

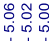

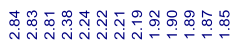

$1 \mathrm{a}$

(400 MHz, $\mathrm{CDCl}_{3}$ )
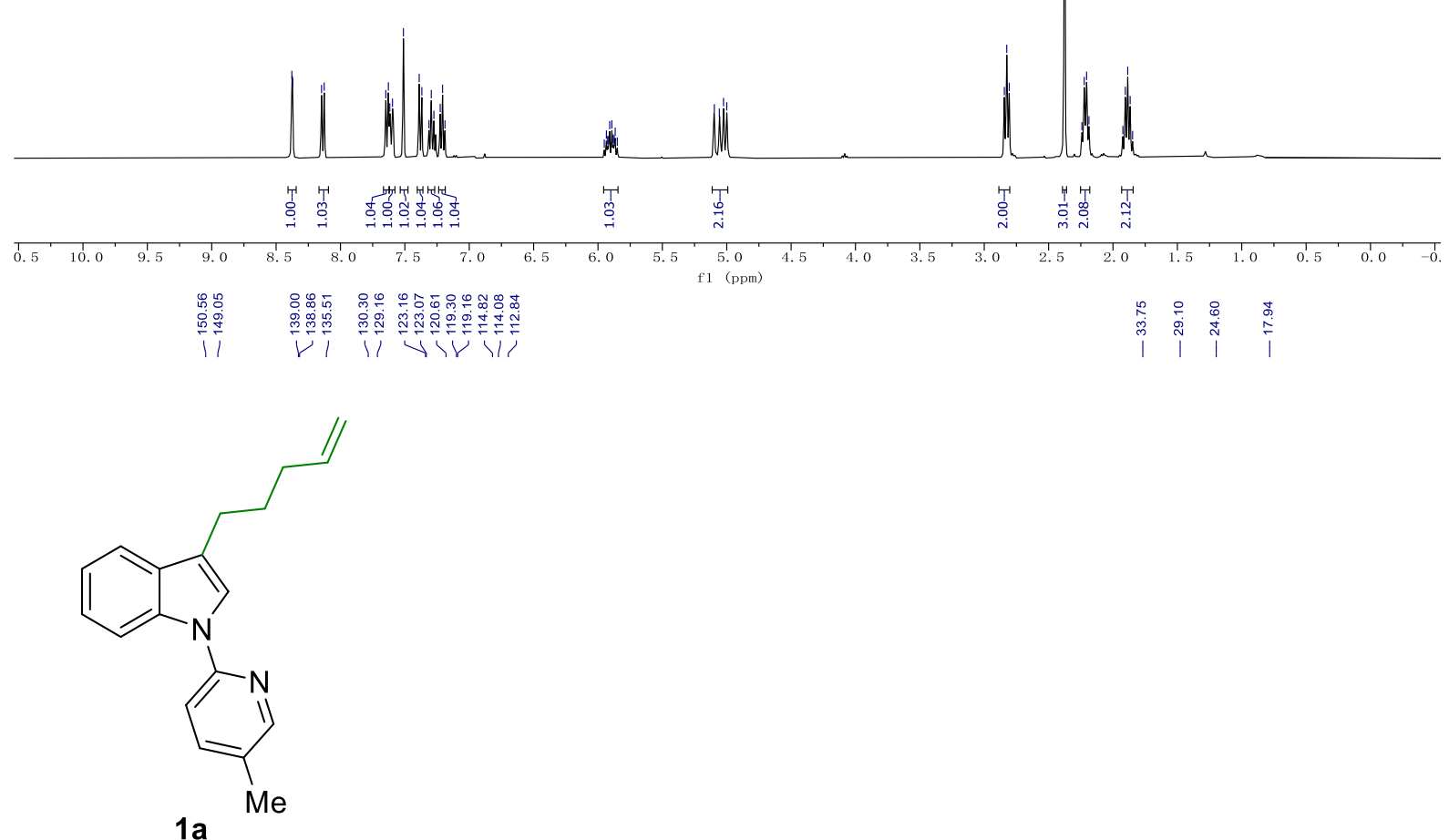

$\left(100 \mathrm{MHz}, \mathrm{CDCl}_{3}\right.$ )
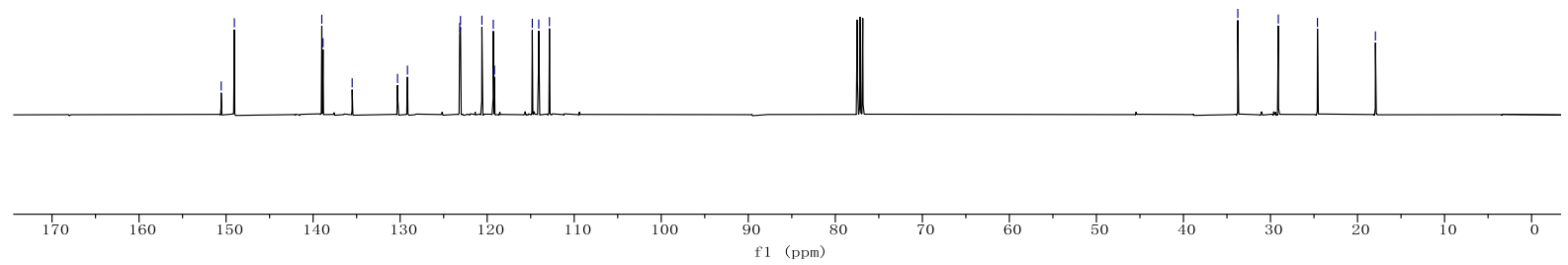


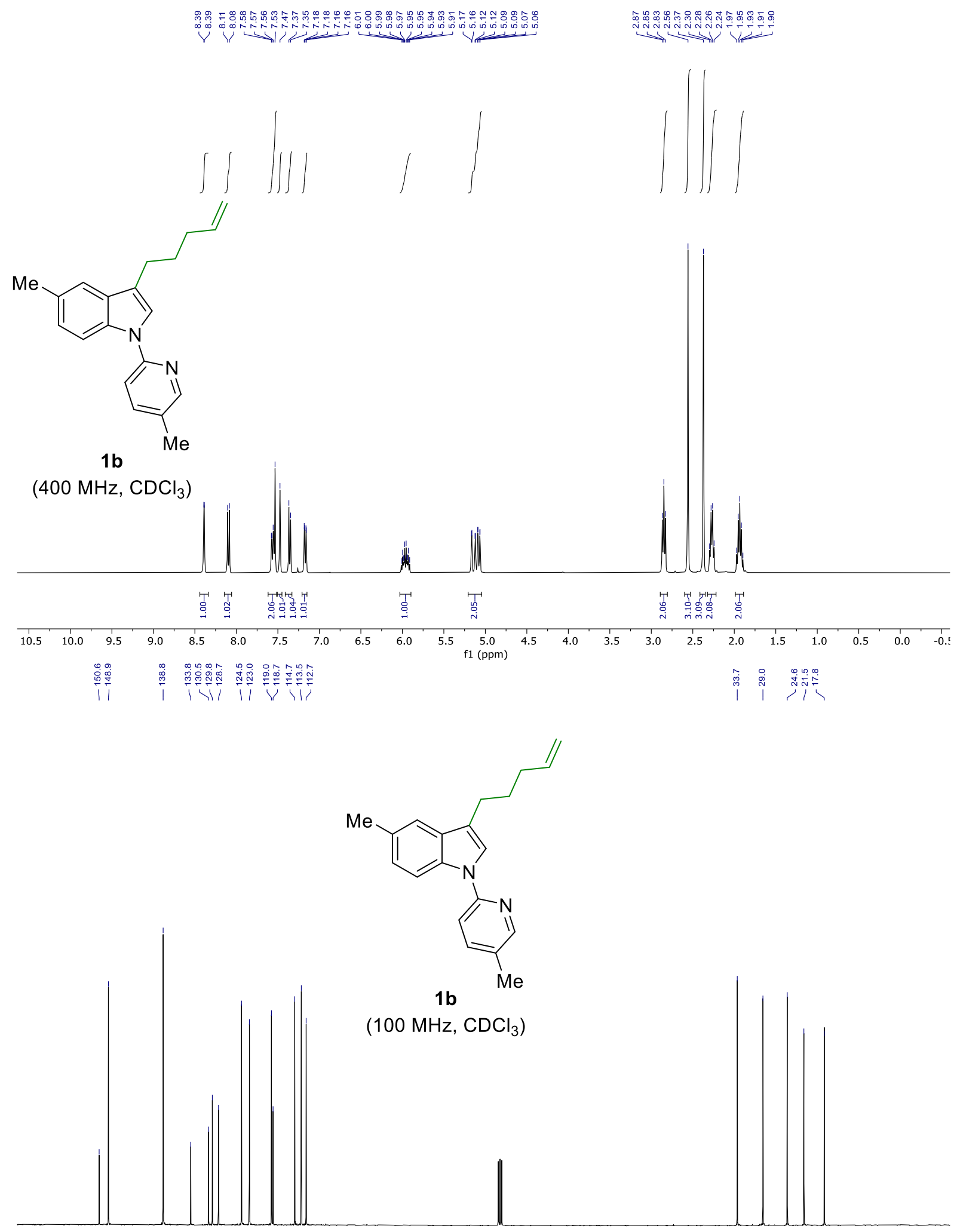

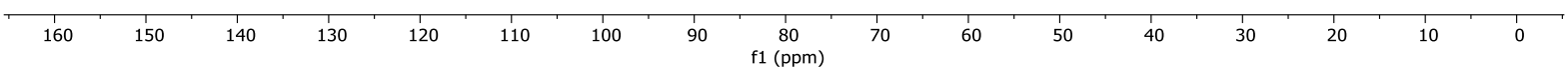



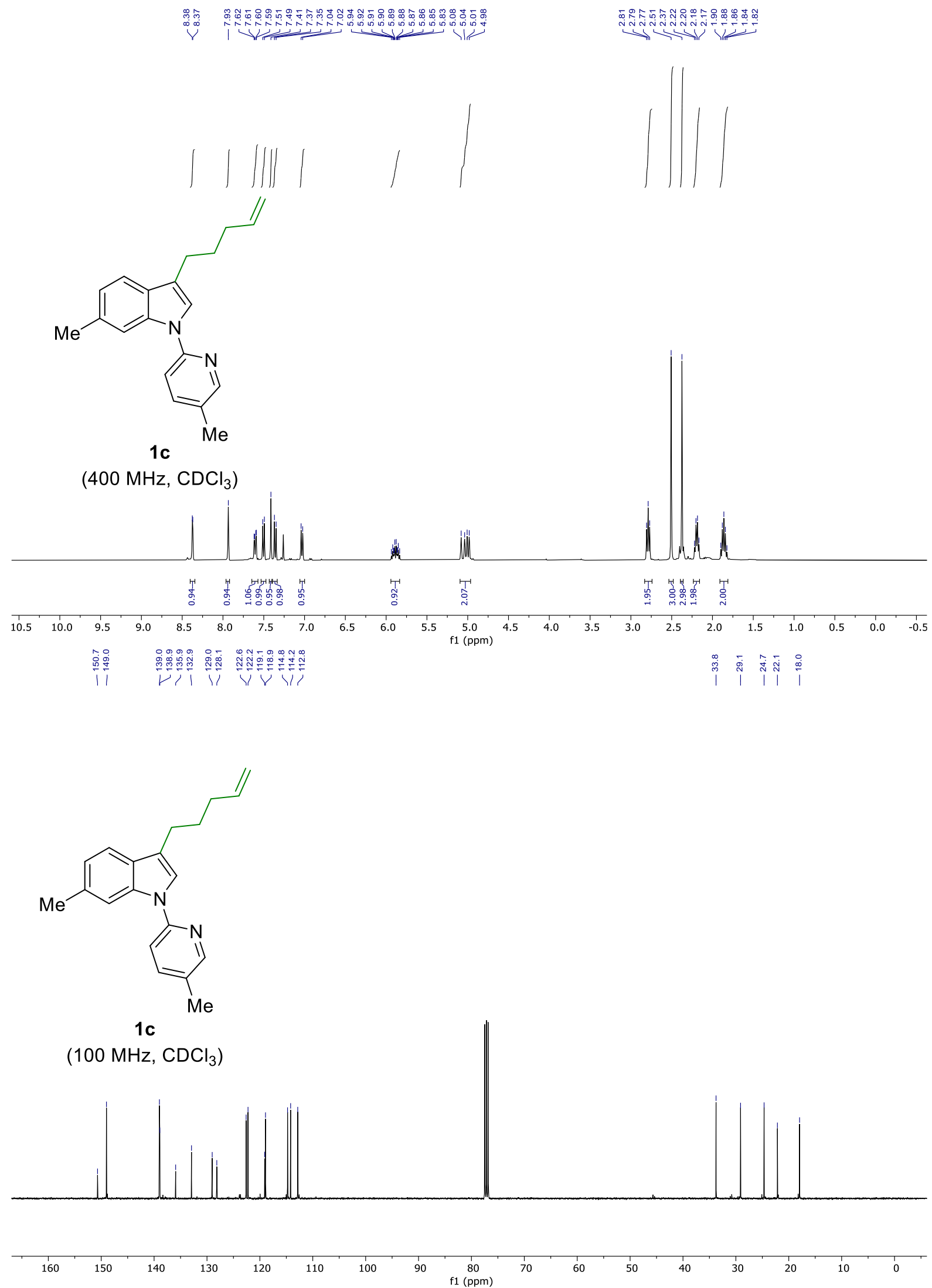


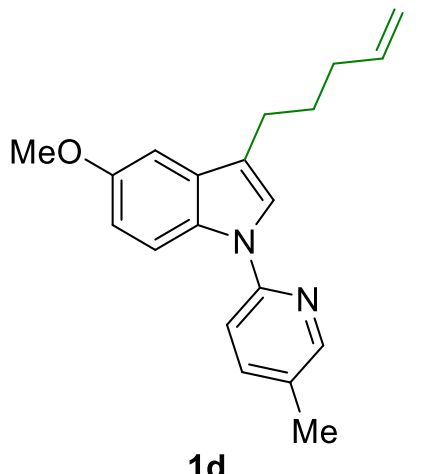

(400 MHz, $\mathrm{CDCl}_{3}$ )
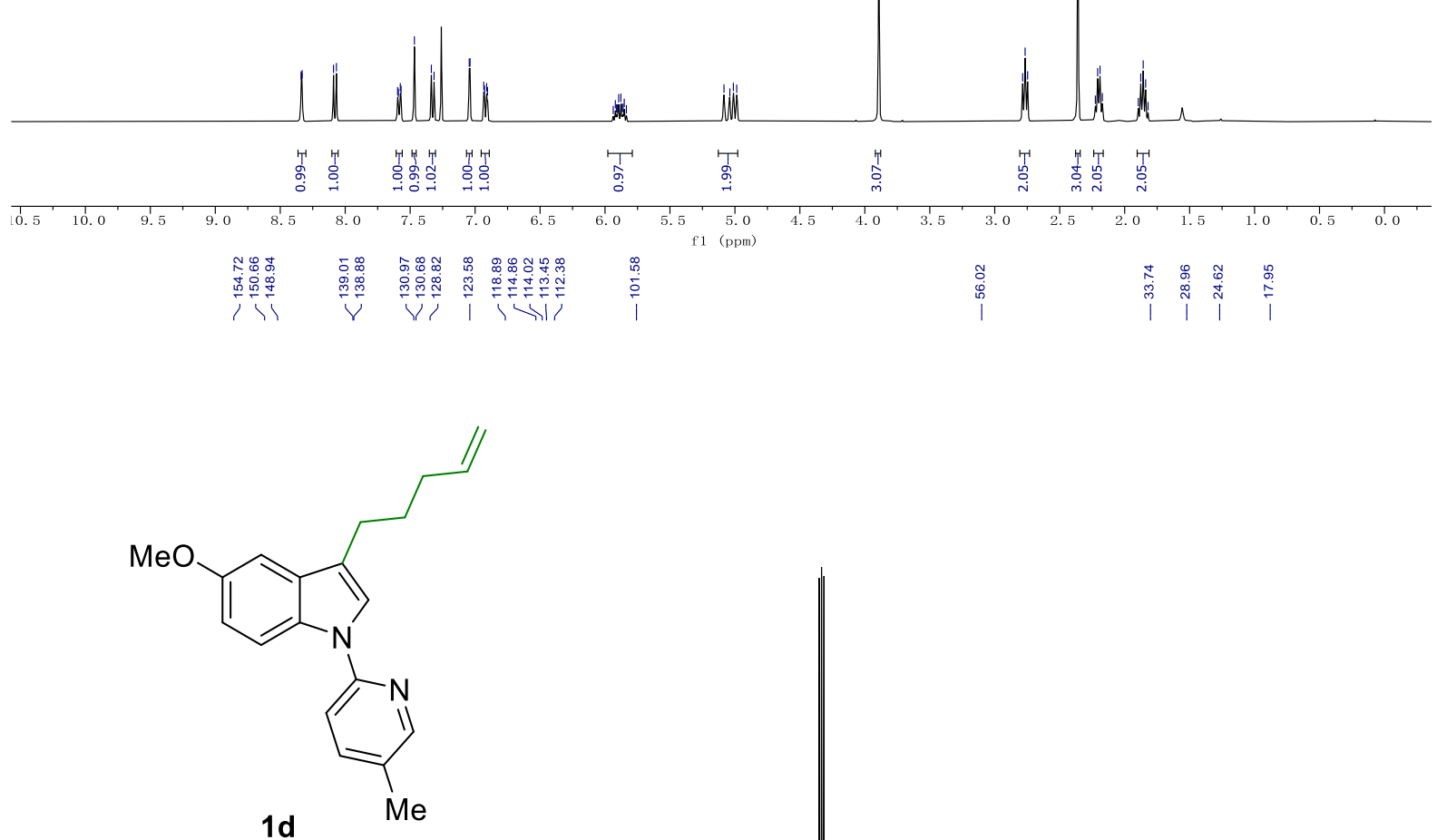

$\left(100 \mathrm{MHz}, \mathrm{CDCl}_{3}\right)$
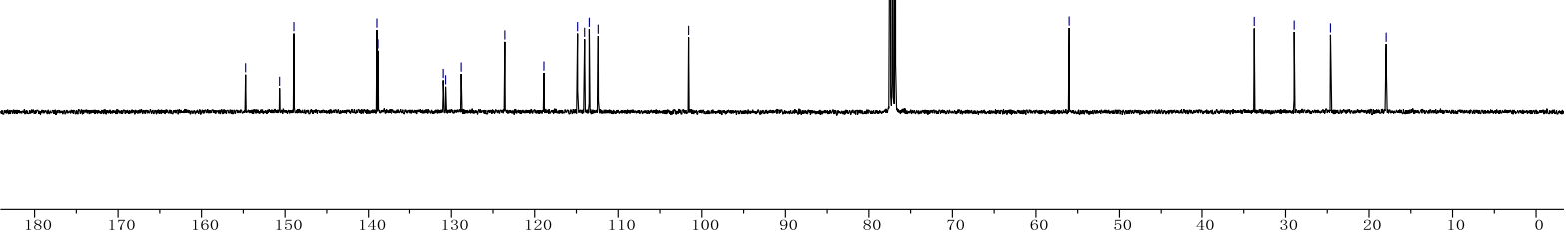

1

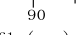




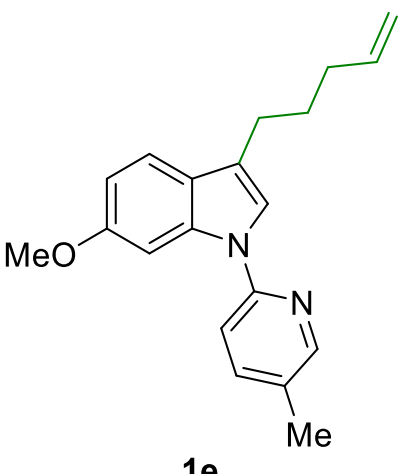

(400 MHz, $\mathrm{CDCl}_{3}$ )
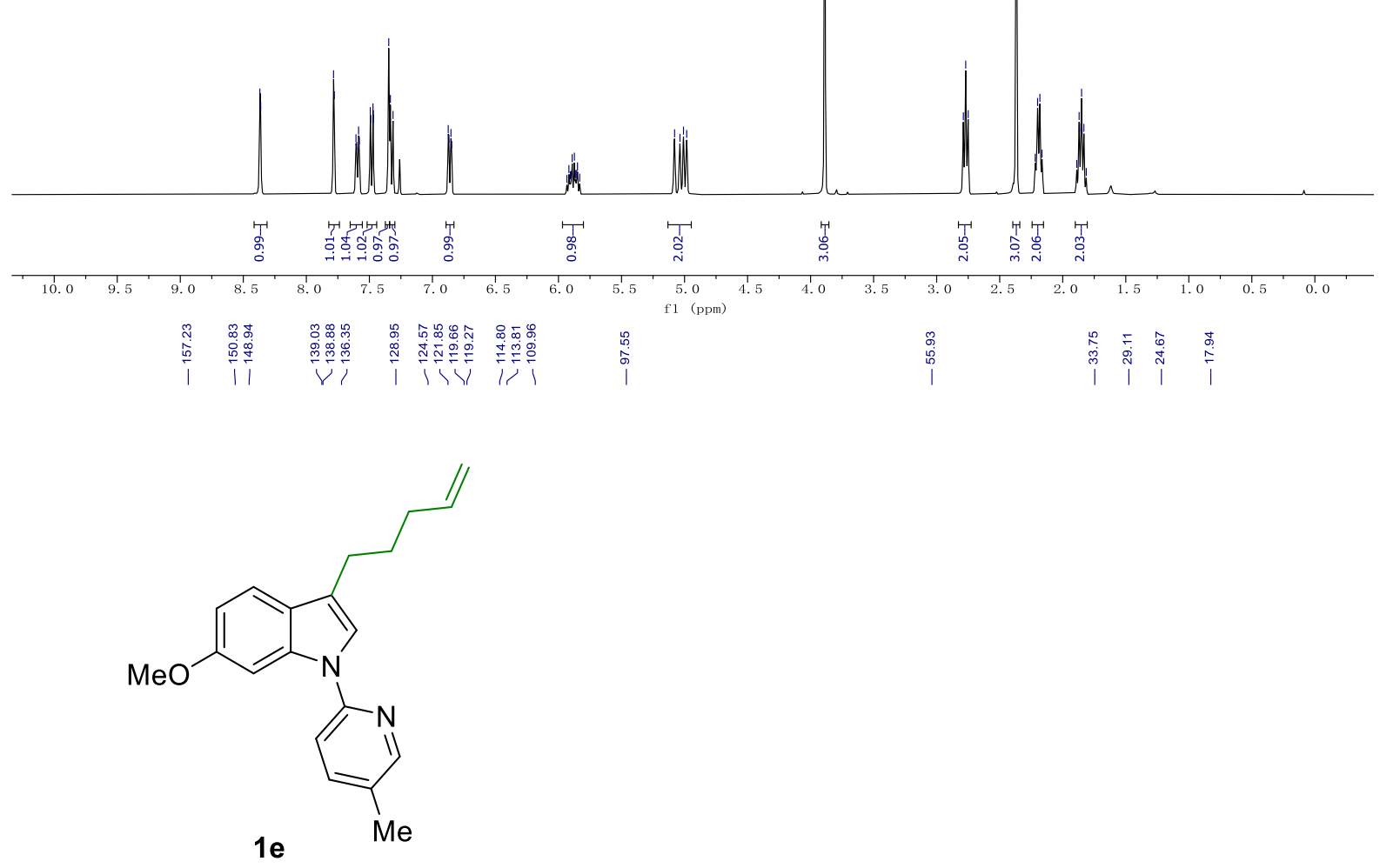

(100 MHz, $\mathrm{CDCl}_{3}$ )

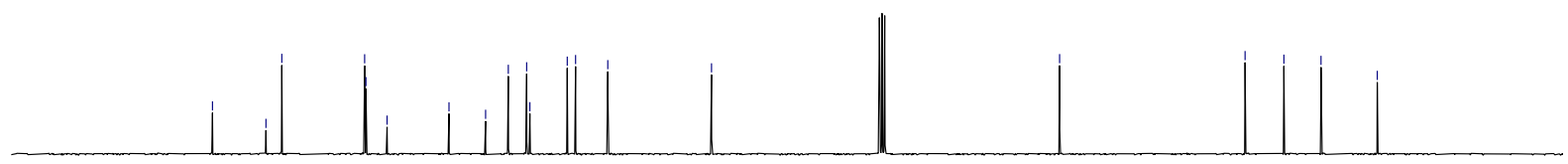

$\frac{1}{180}$

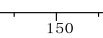

90
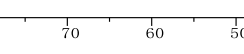


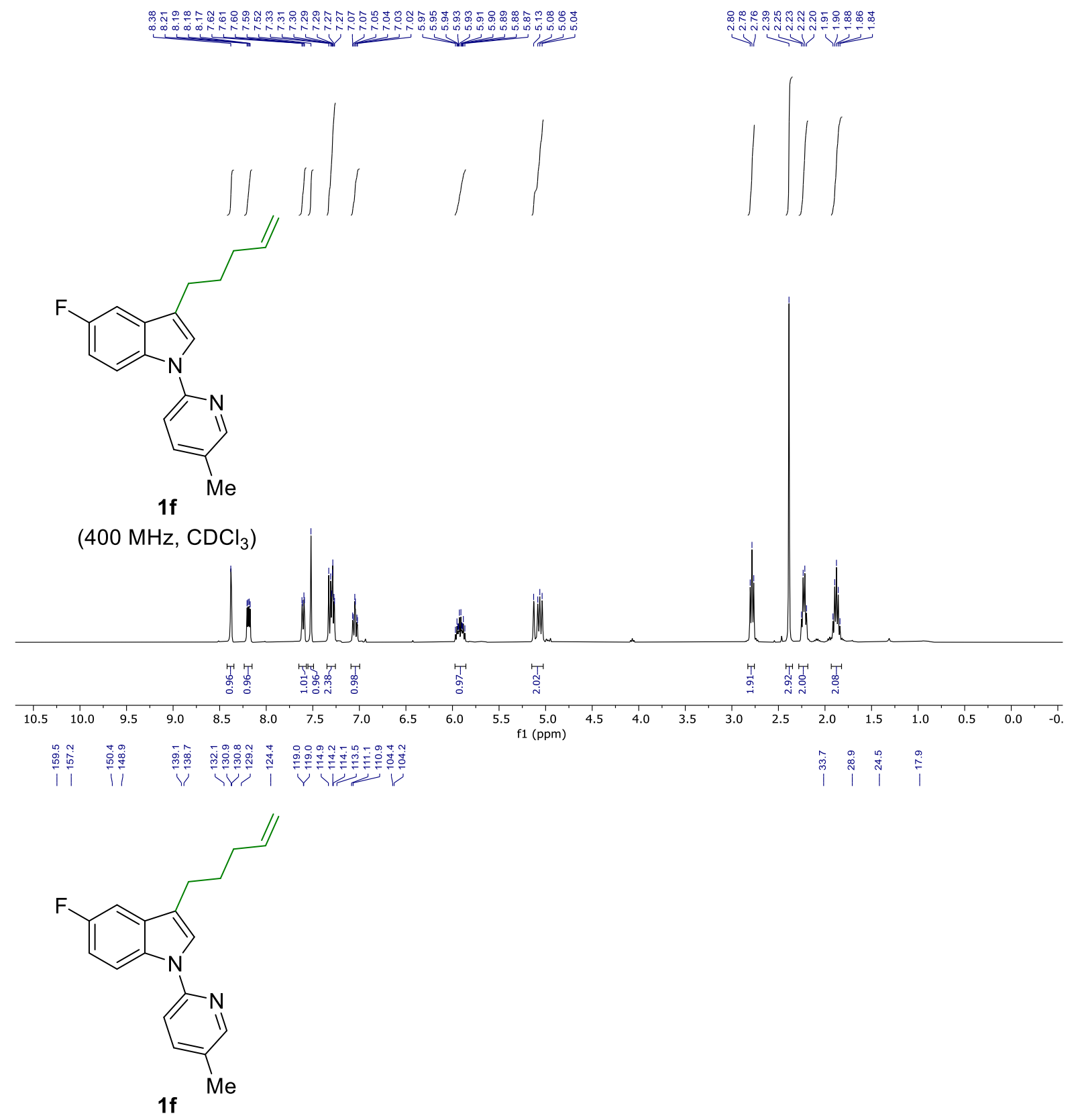

$\left(100 \mathrm{MHz}, \mathrm{CDCl}_{3}\right)$

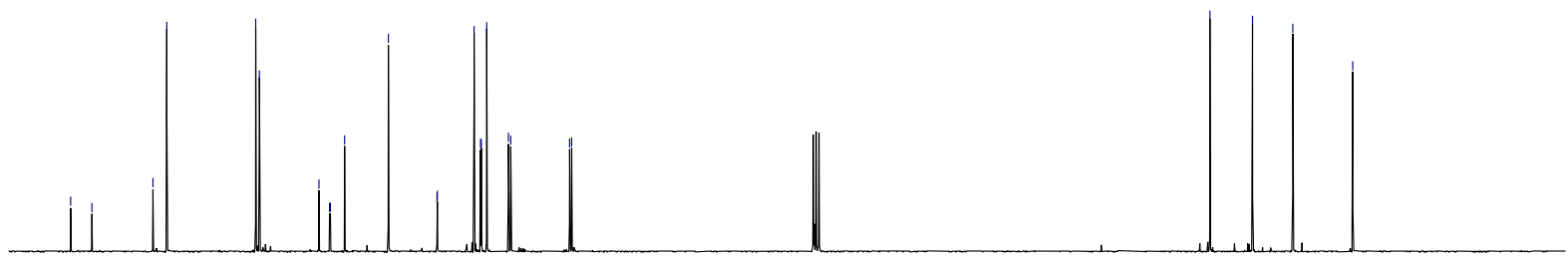

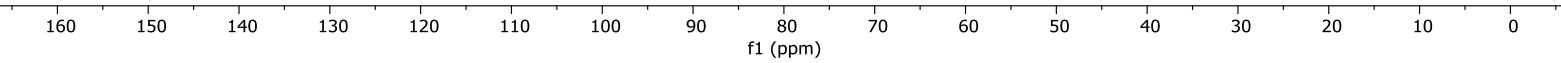




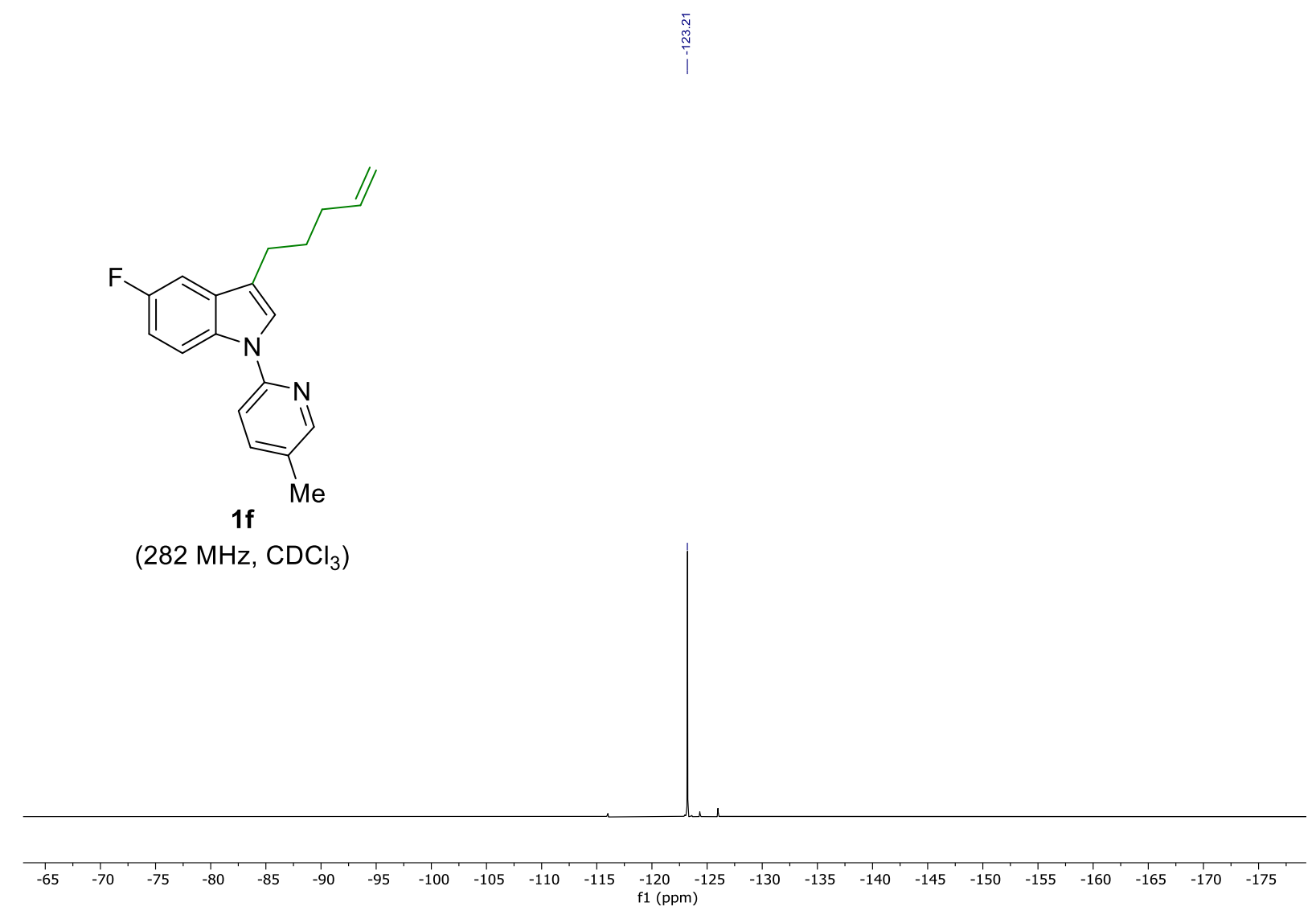




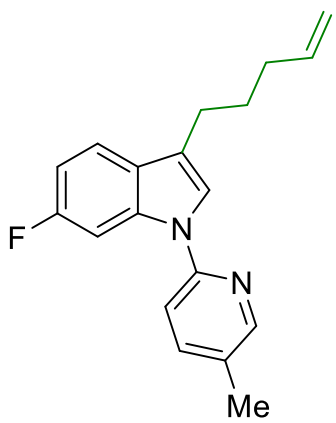

$1 \mathrm{~g}$

$\left(400 \mathrm{MHz}, \mathrm{CDCl}_{3}\right)$
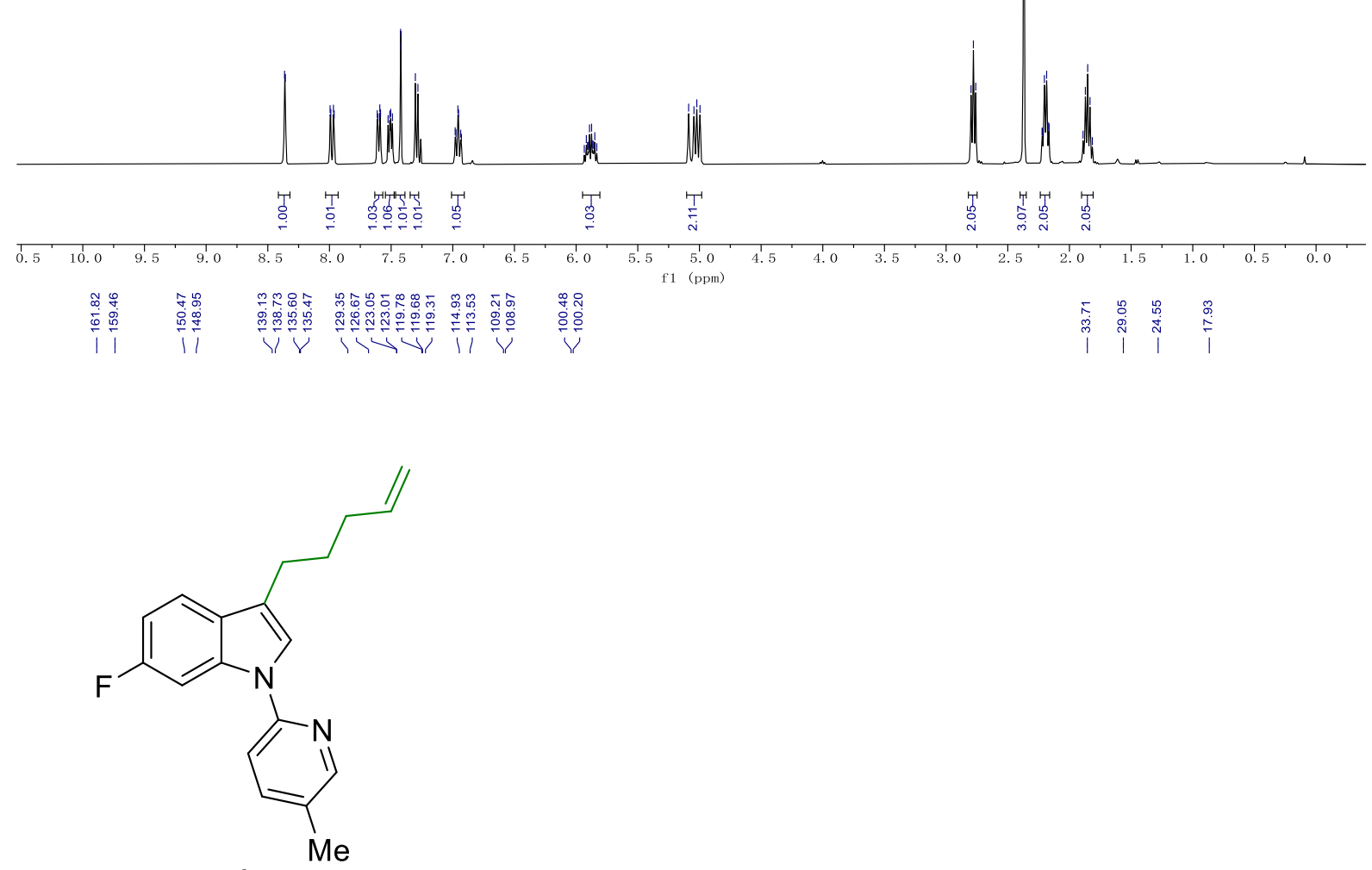

$1 \mathrm{~g}$

(100 MHz, $\mathrm{CDCl}_{3}$ )

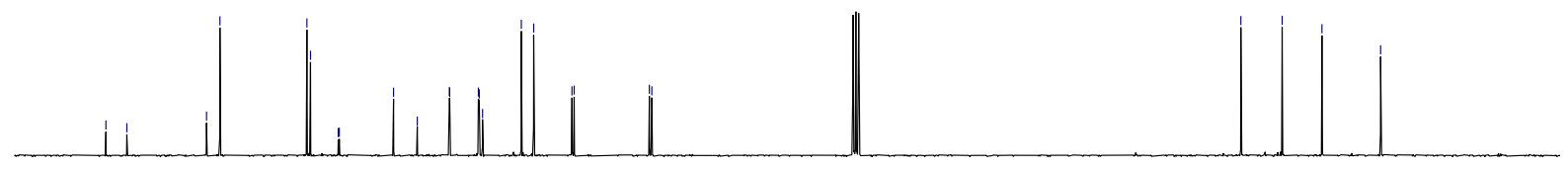

170

160
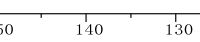

$90+80$ 


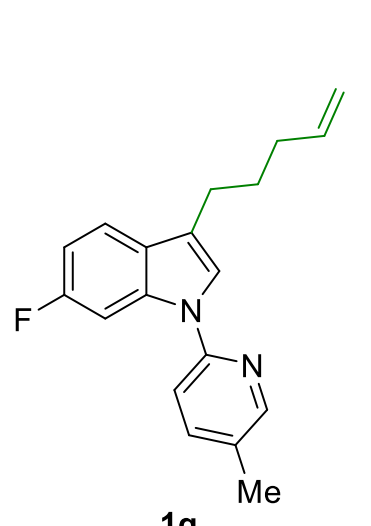

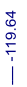

$\left(282 \mathrm{MHz}, \mathrm{CDCl}_{3}\right)$

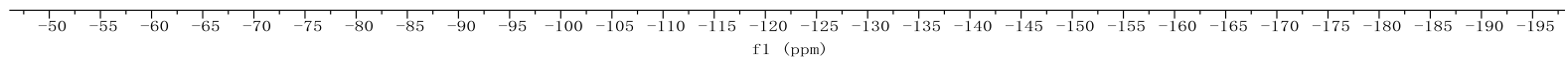



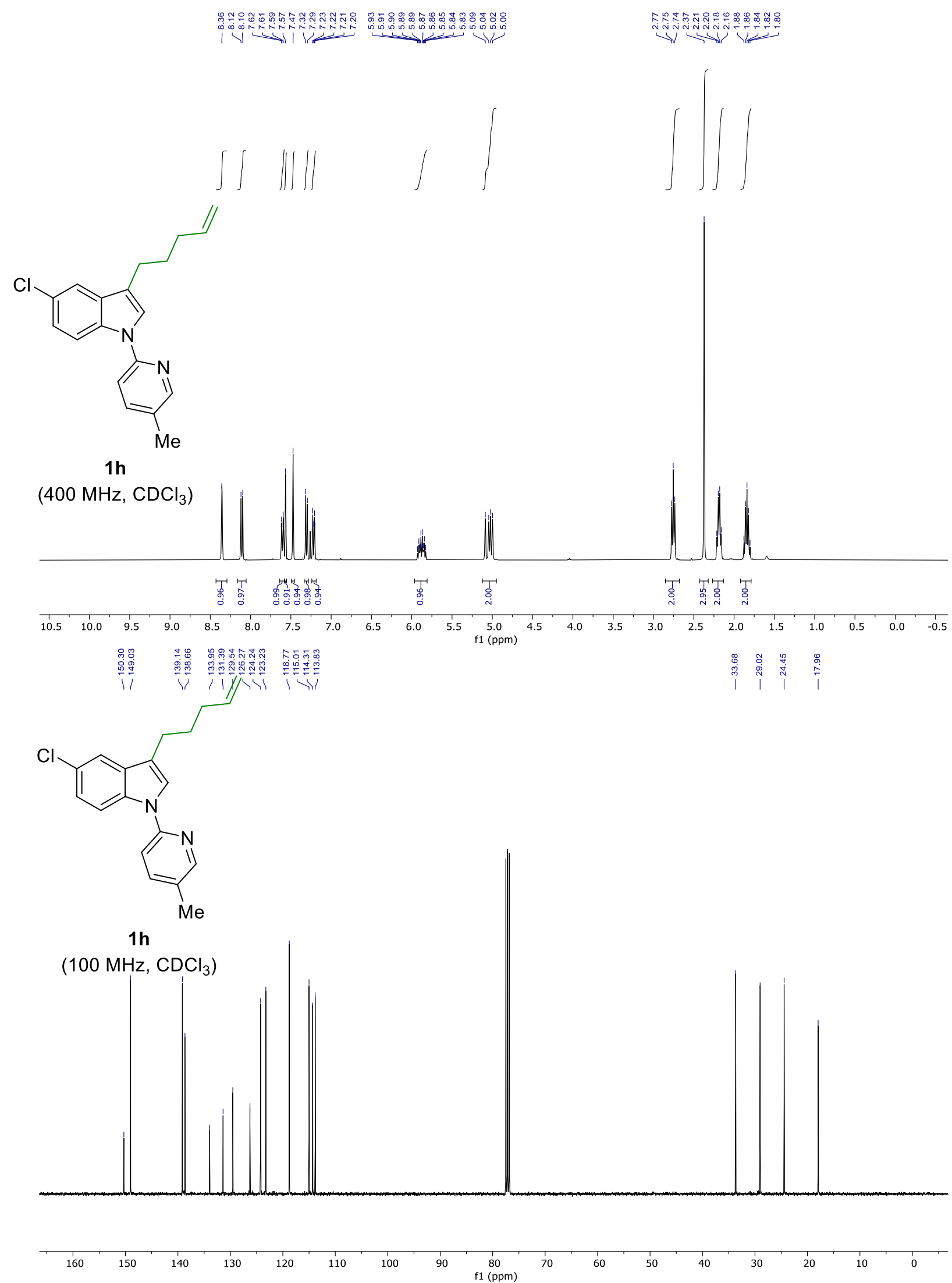

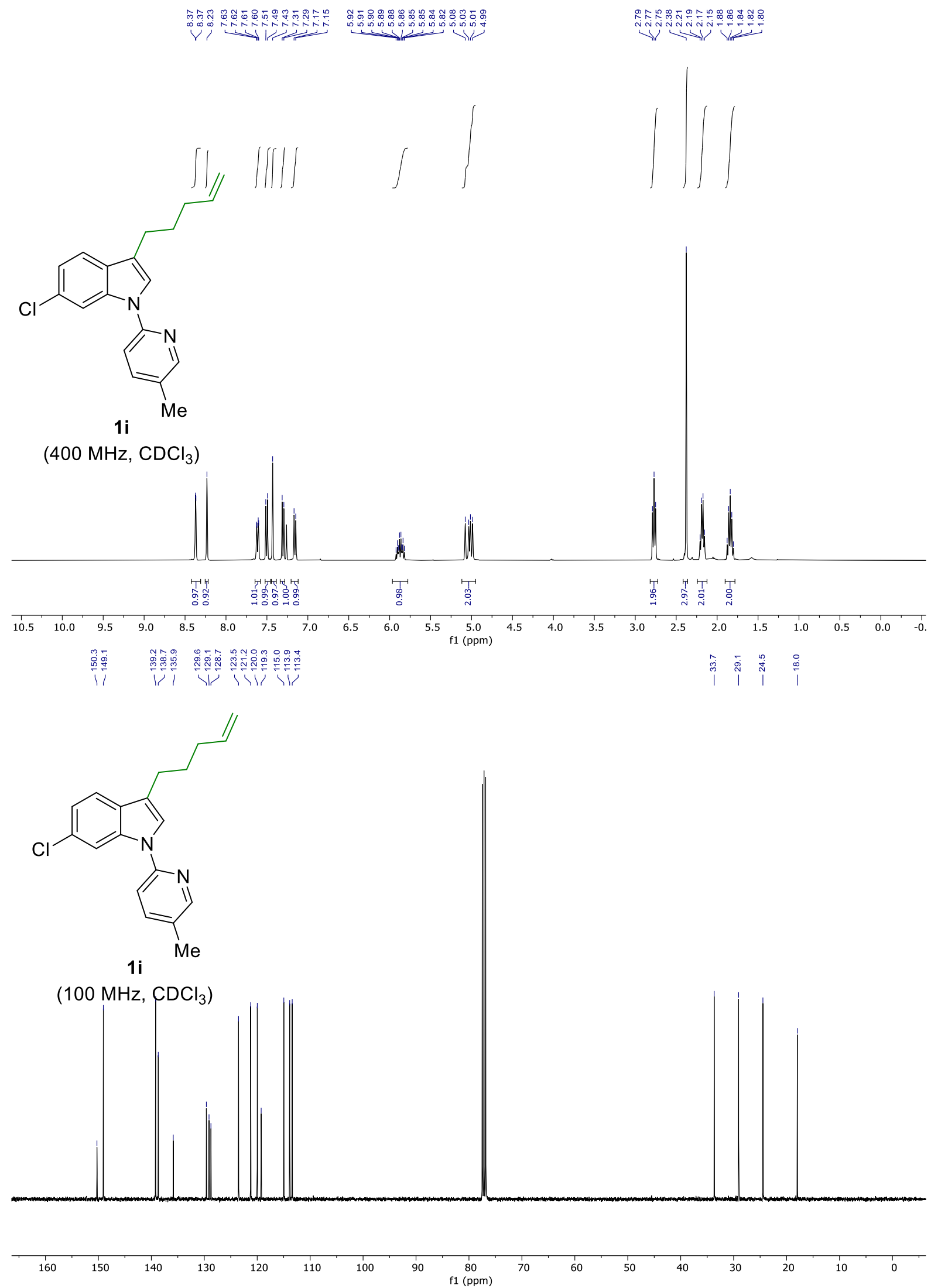

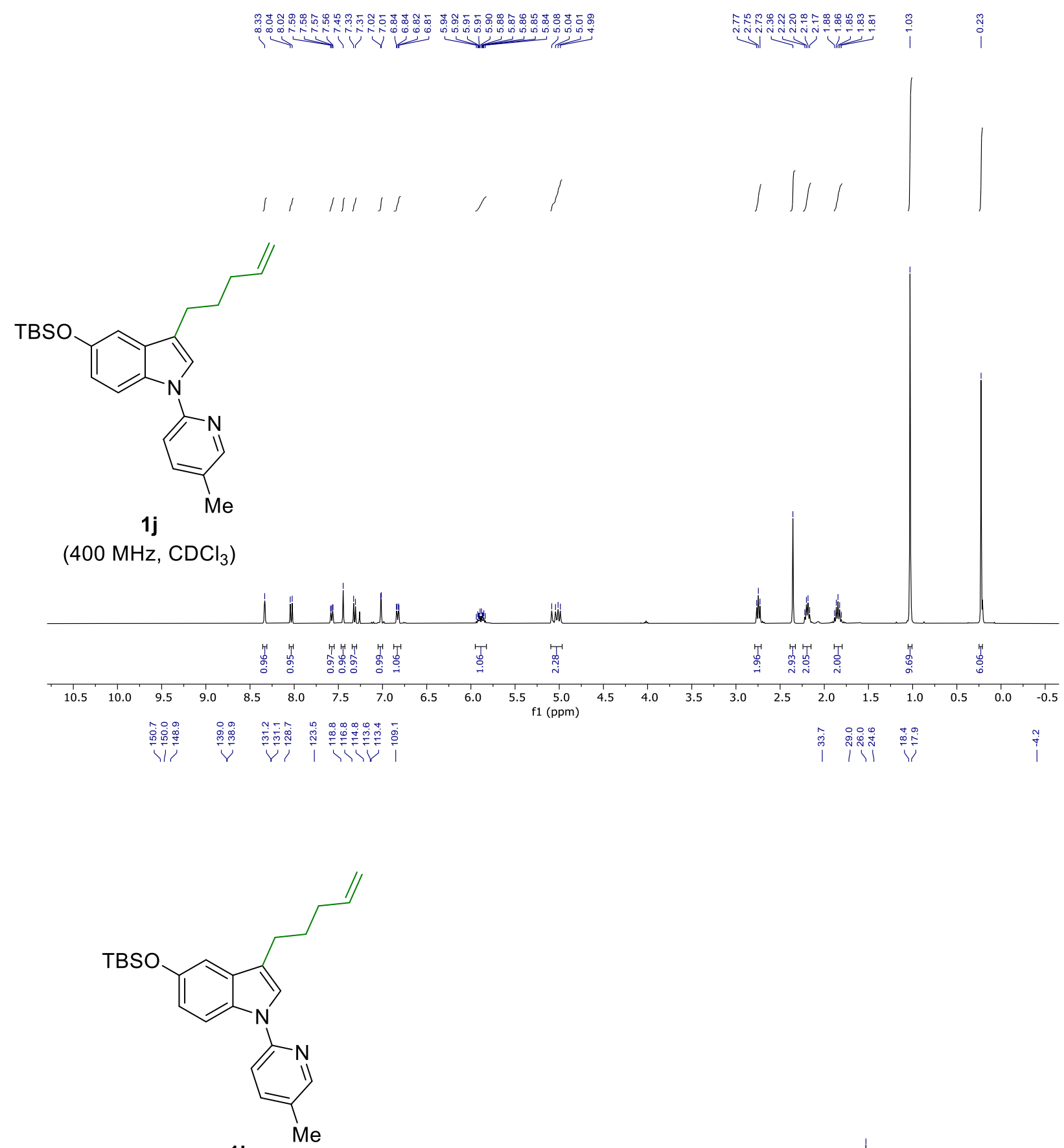

1j

(100 MHz, $\mathrm{CDCl}_{3}$ )
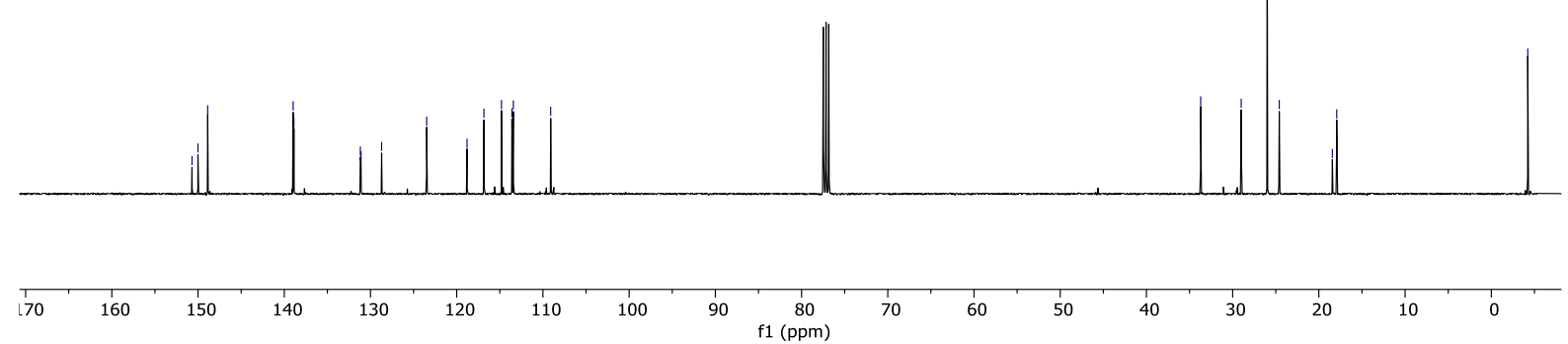


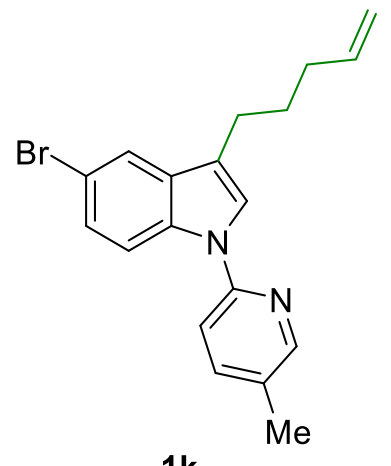

$1 \mathrm{k}$

$\left(400 \mathrm{MHz}, \mathrm{CDCl}_{3}\right)$
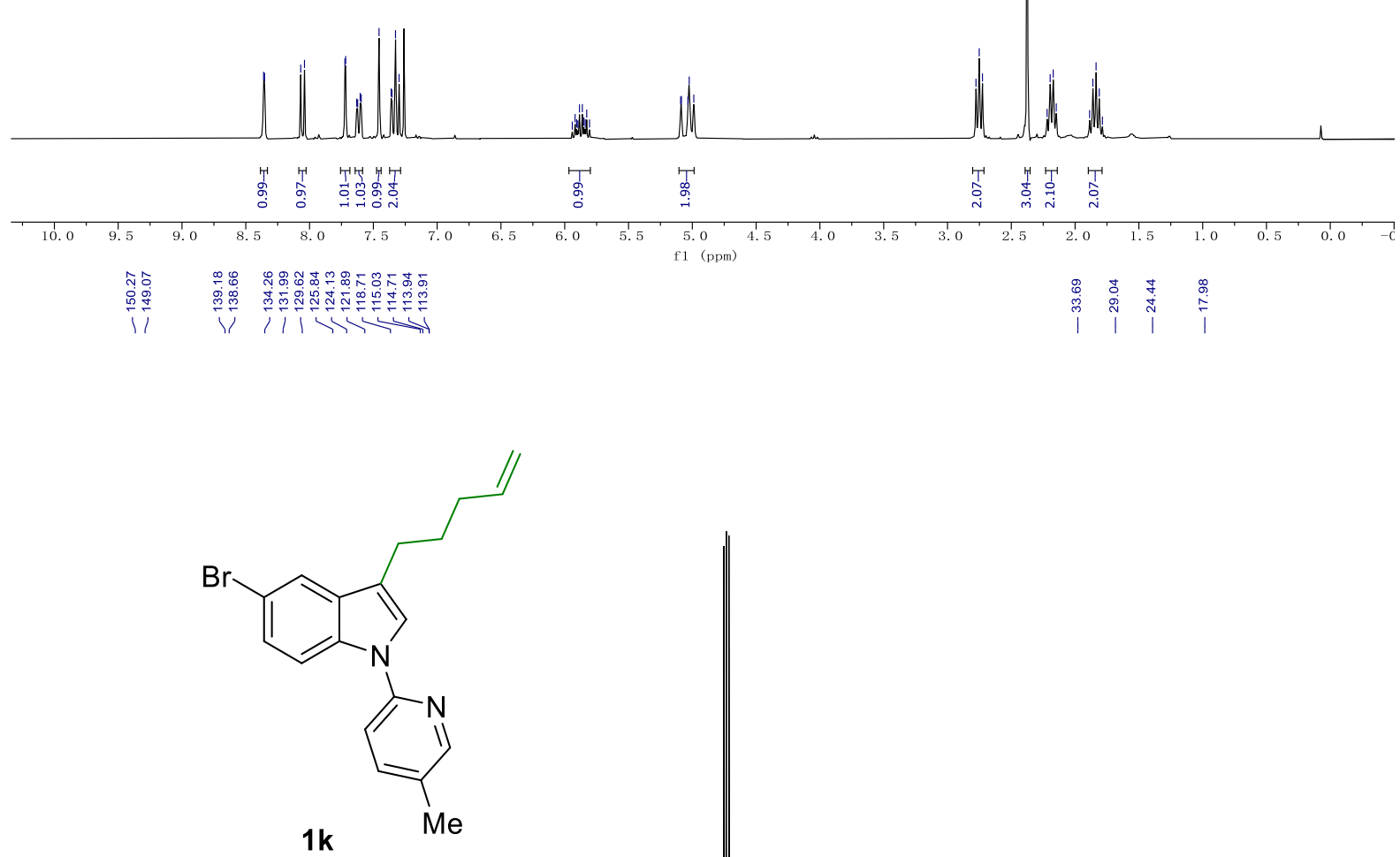

(100 MHz, $\mathrm{CDCl}_{3}$ )
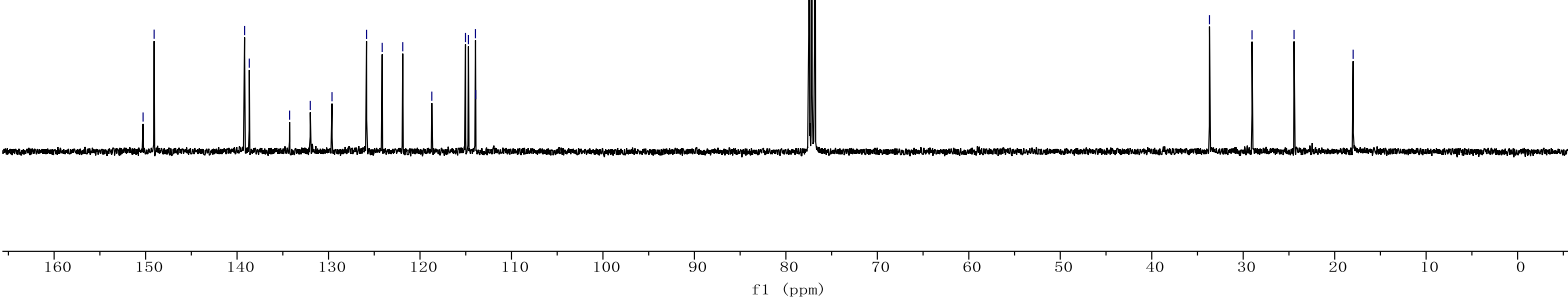

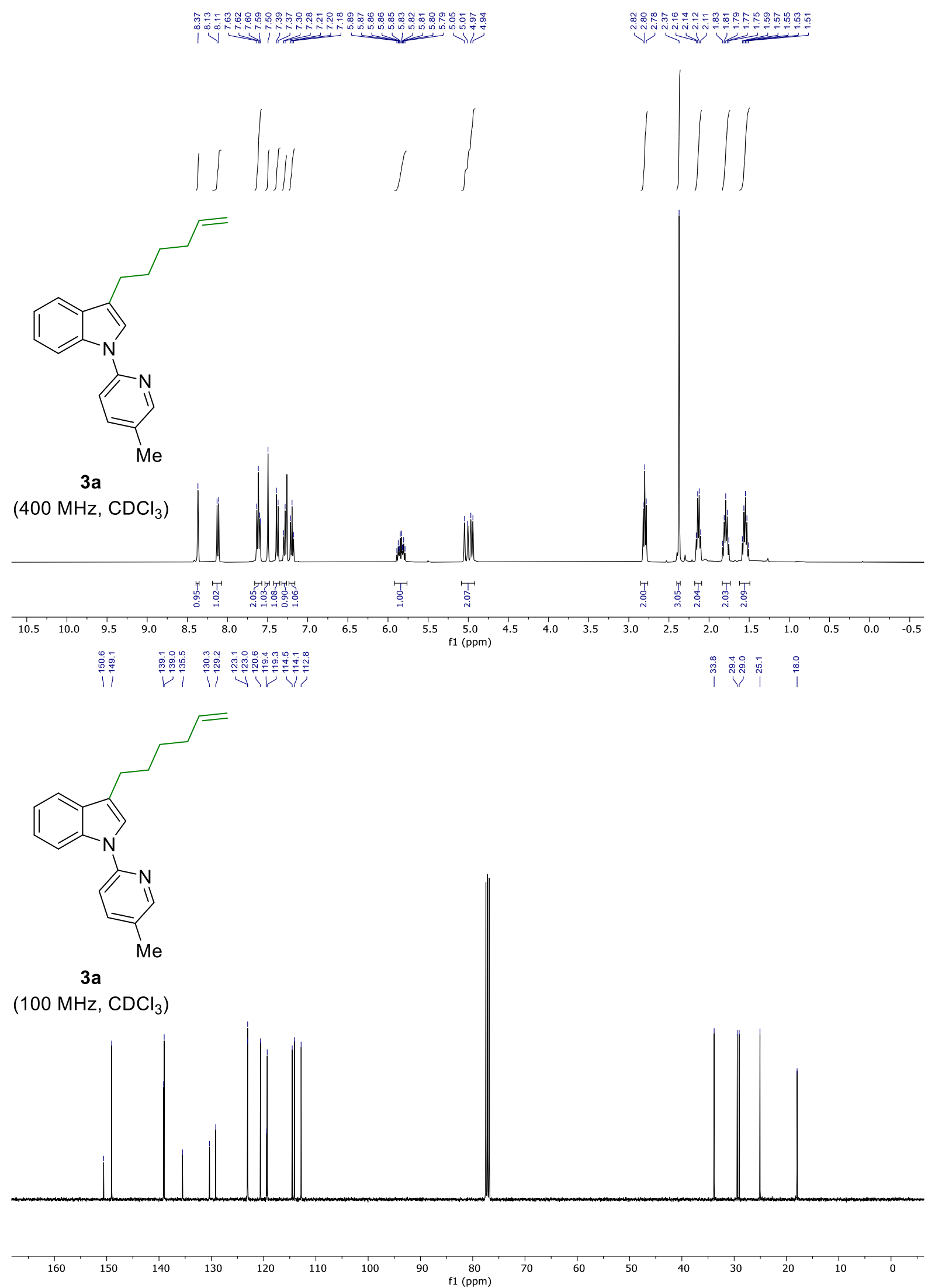


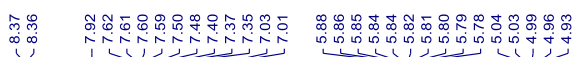

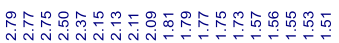

N V N N
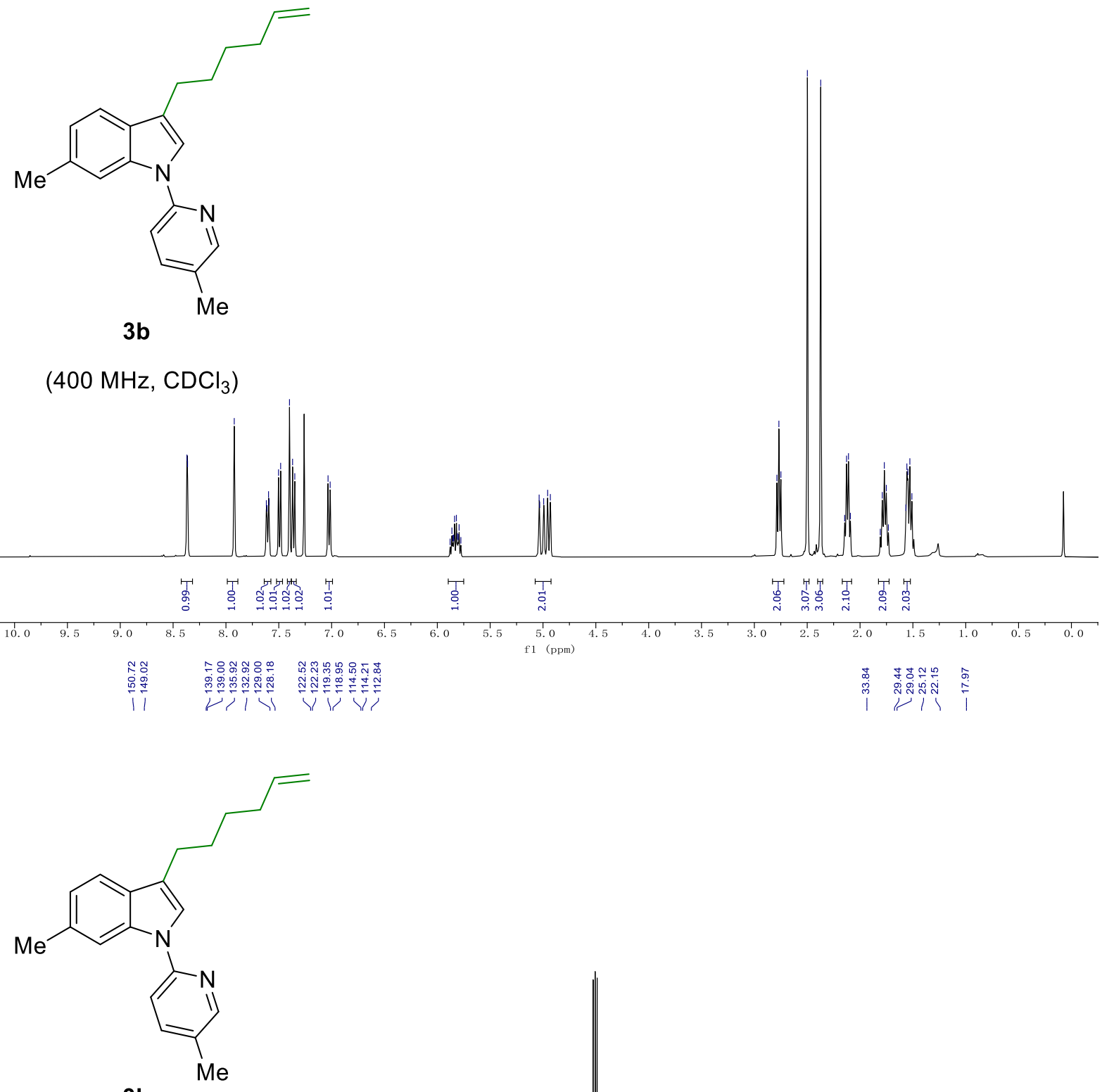

$3 b$

(100 MHz, $\mathrm{CDCl}_{3}$ )
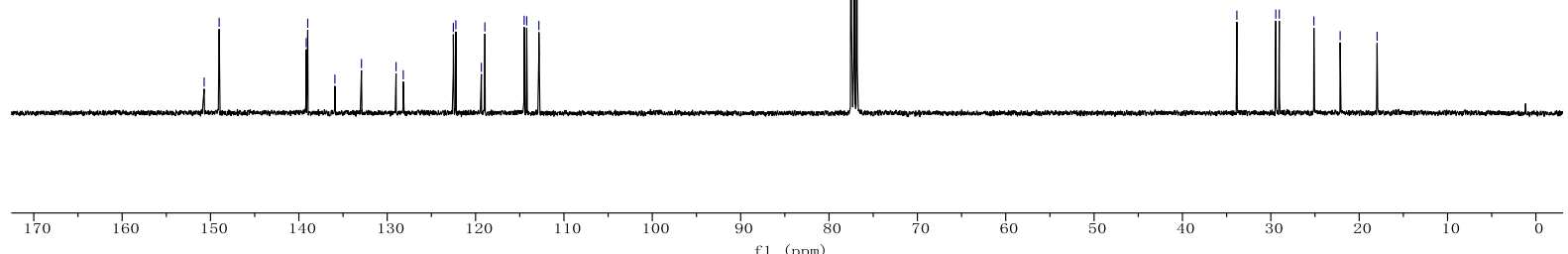

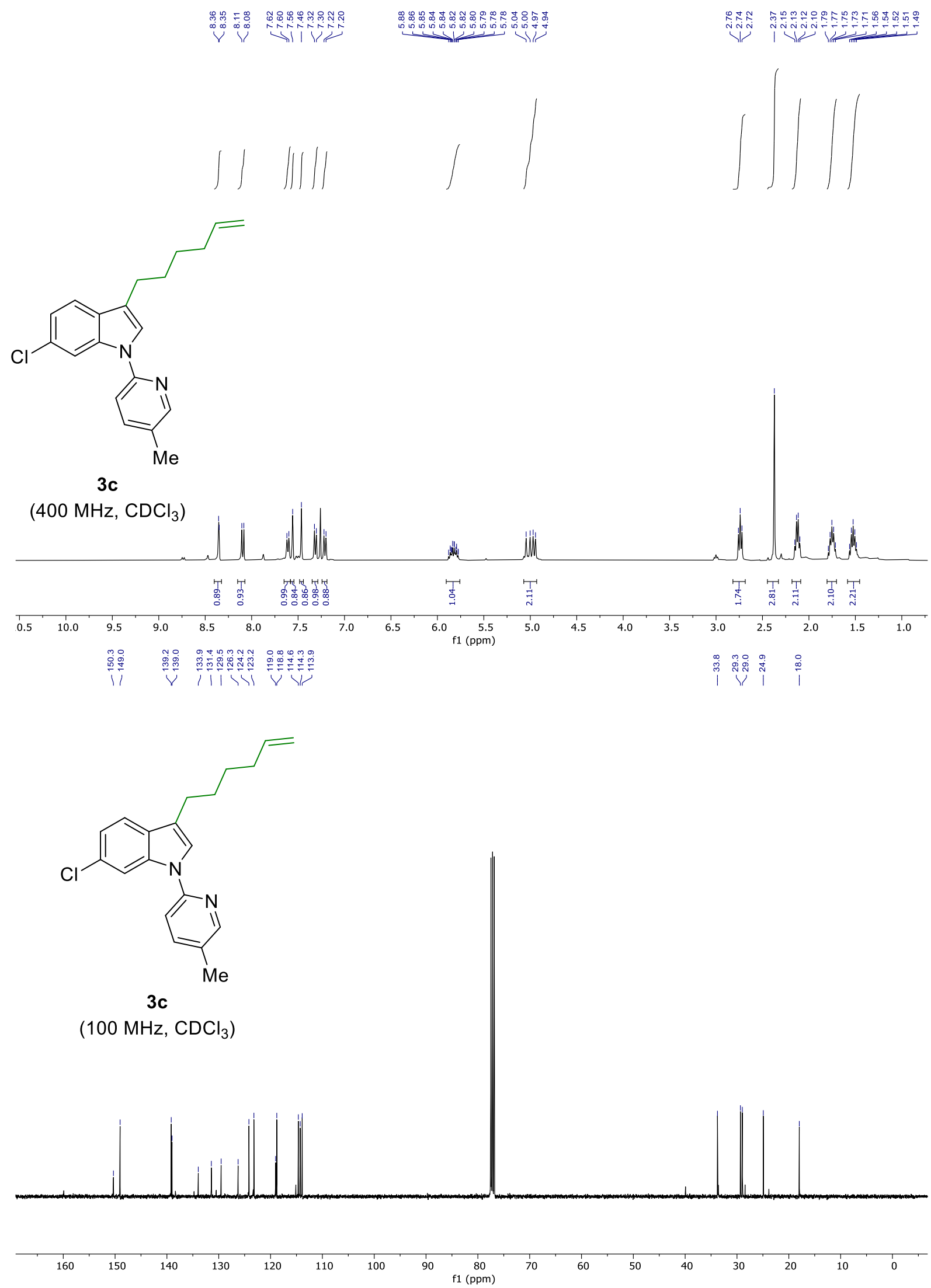

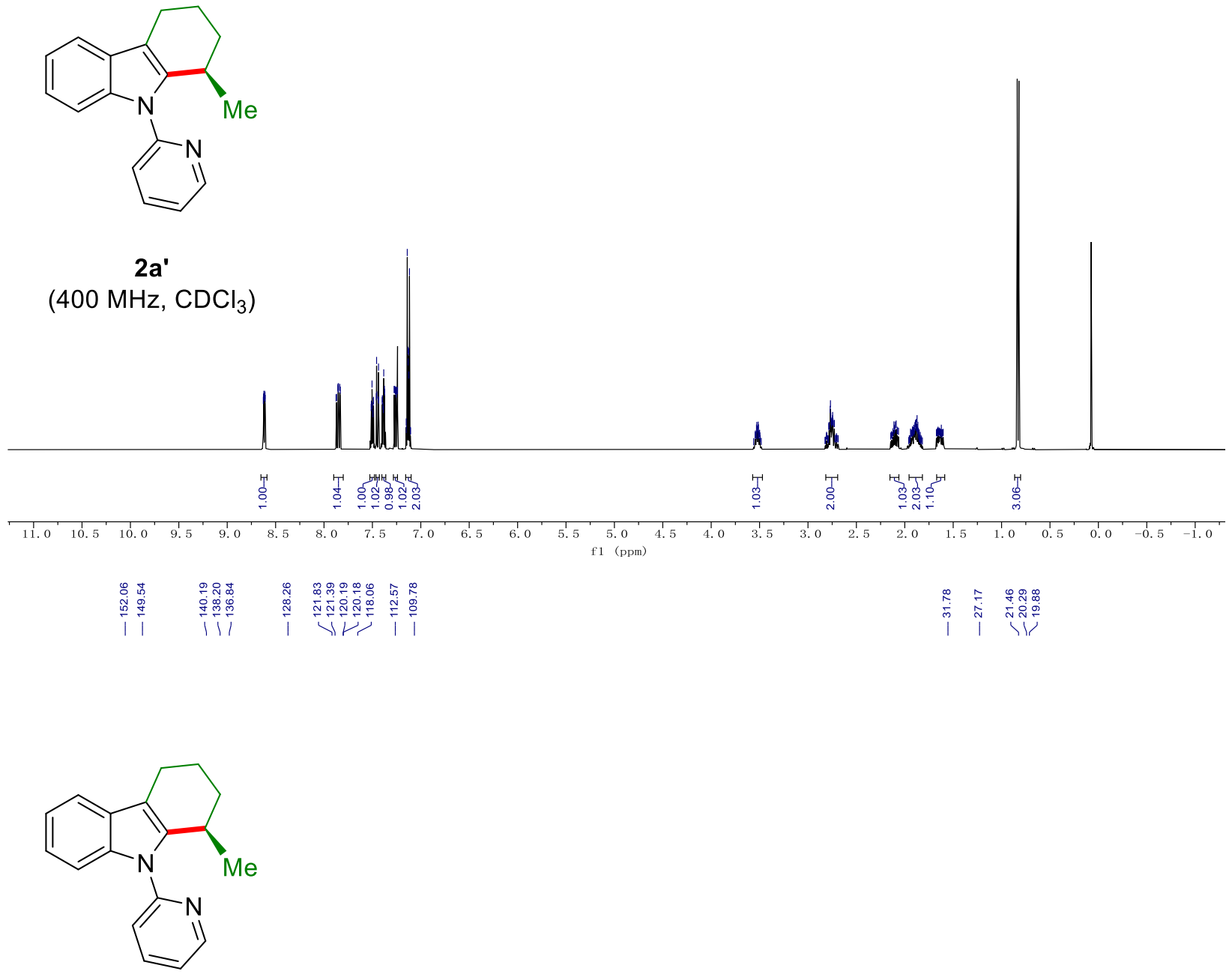

2a'

$\left(100 \mathrm{MHz}, \mathrm{CDCl}_{3}\right.$ )
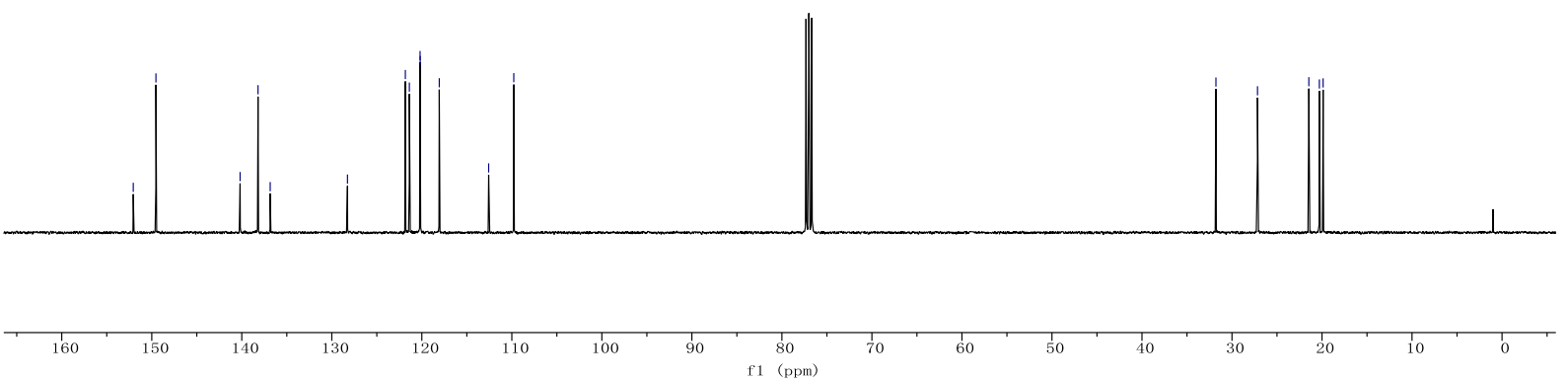

S112 

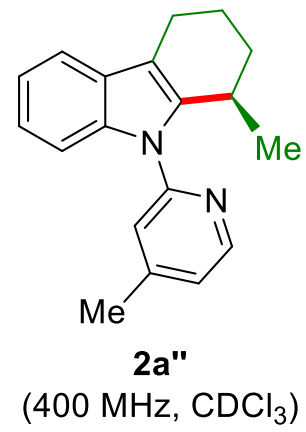

$\left(400 \mathrm{MHz} \mathrm{CDCl}_{3}\right)$

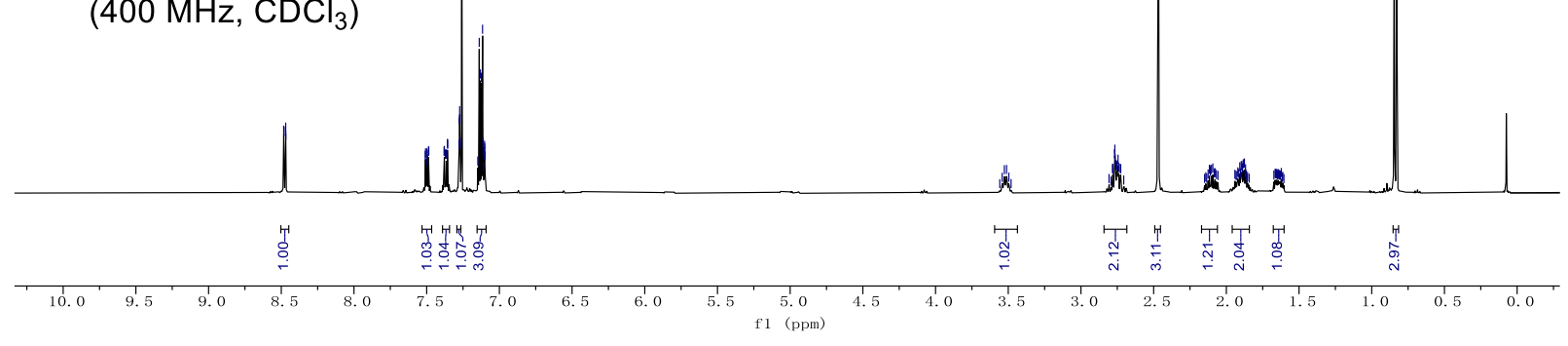
|l|

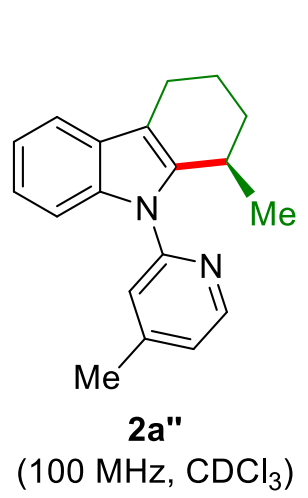

2a"

$\left(100 \mathrm{MHz}, \mathrm{CDCl}_{3}\right.$ )

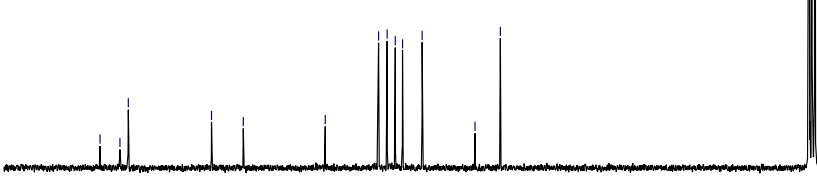

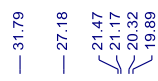

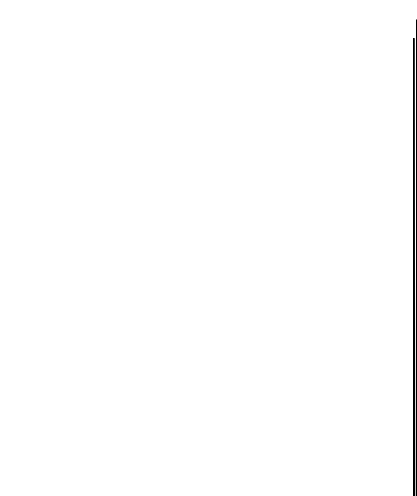




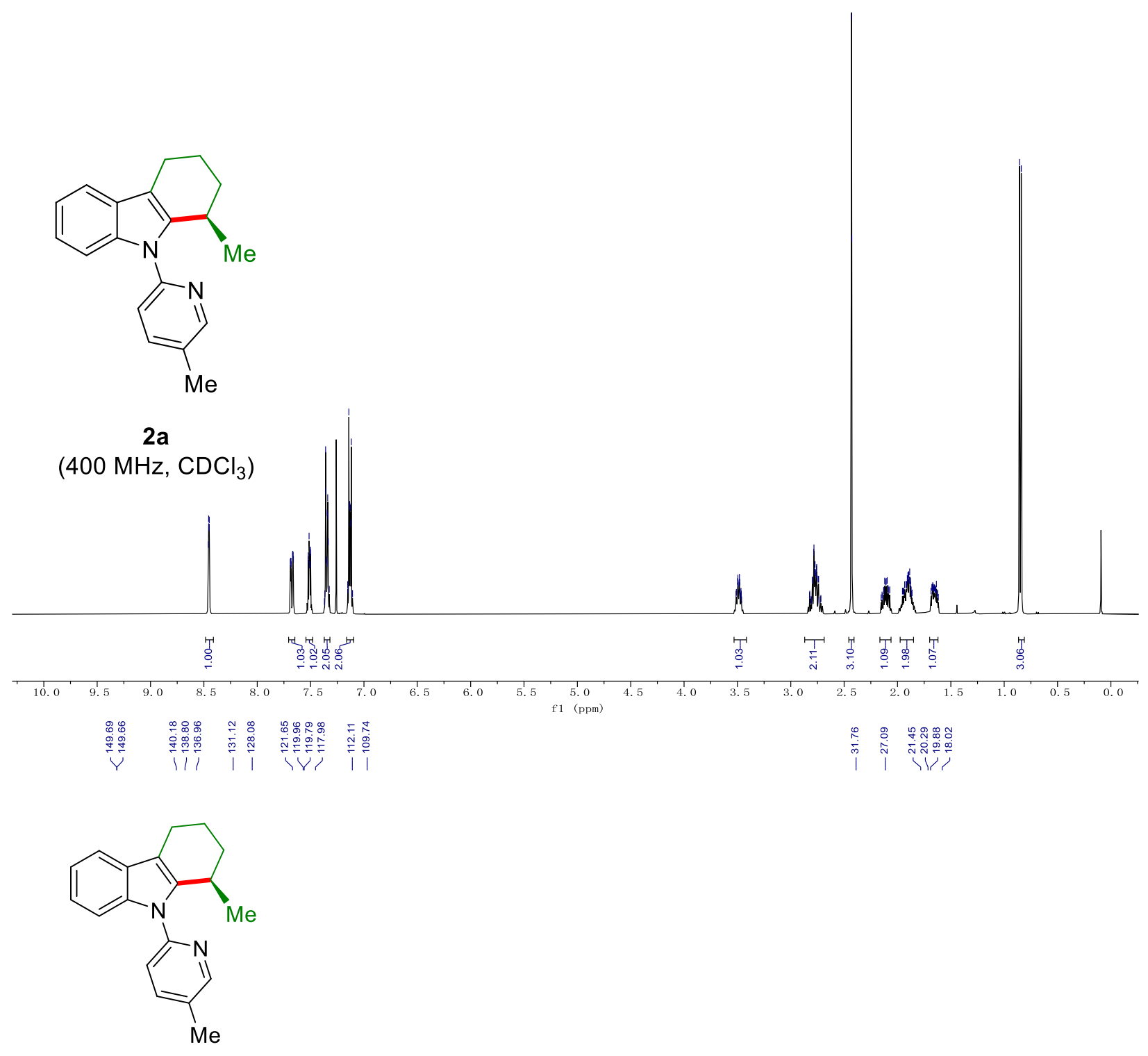

2a

(100 MHz, $\mathrm{CDCl}_{3}$ )
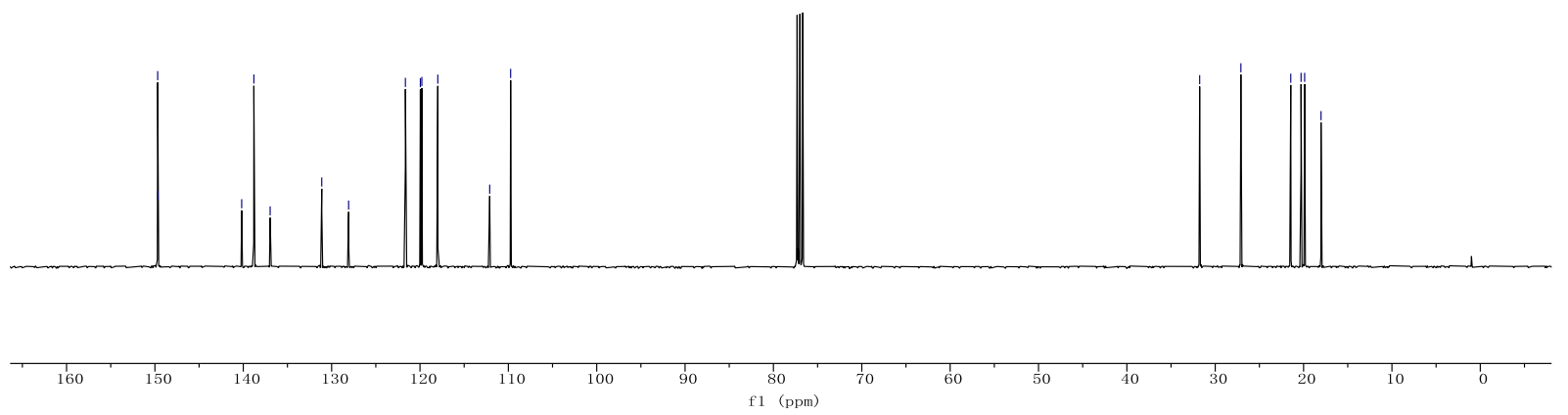
<smiles>[CH]C1CCCc2c1n(-c1ccc(C)cn1)c1ccc(C)cc21</smiles>

2b

$\left(300 \mathrm{MHz}, \mathrm{CDCl}_{3}\right)$<smiles>[CH]C1CCCc2c1n(-c1ccc(C)cn1)c1ccc(C)cc21</smiles>

$2 \mathrm{~b}$

$\left(100 \mathrm{MHz}, \mathrm{CDCl}_{3}\right)$

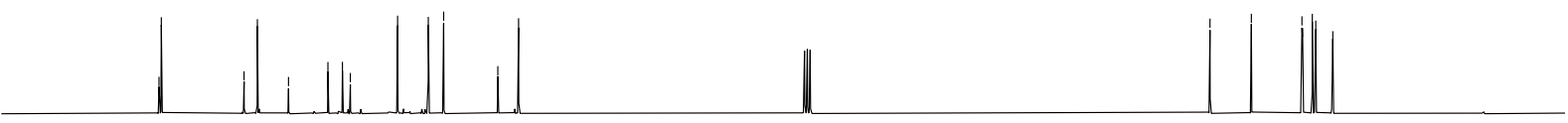

$\begin{array}{llllll}160 & 150 & 140 & 130 & 120 & 110\end{array}$ 


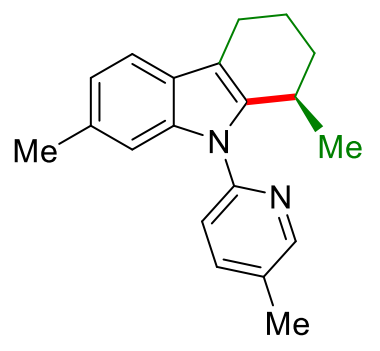

2c

$\left(400 \mathrm{MHz}, \mathrm{CDCl}_{3}\right)$

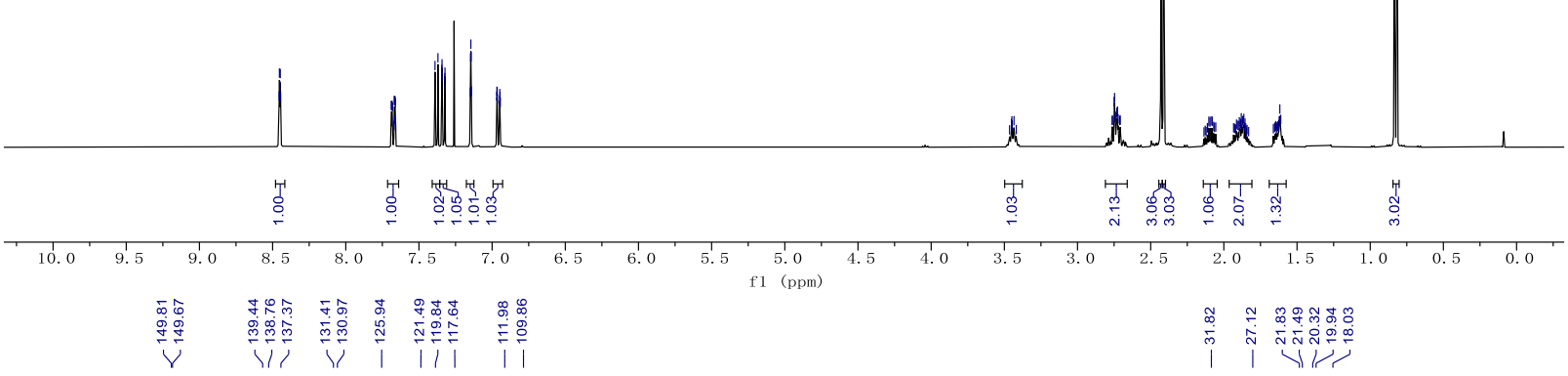<smiles>Cc1ccc(-n2c3c(c4ccc(C)cc42)CCCC3C)nc1</smiles>

2c

$\left(100 \mathrm{MHz}, \mathrm{CDCl}_{3}\right)$

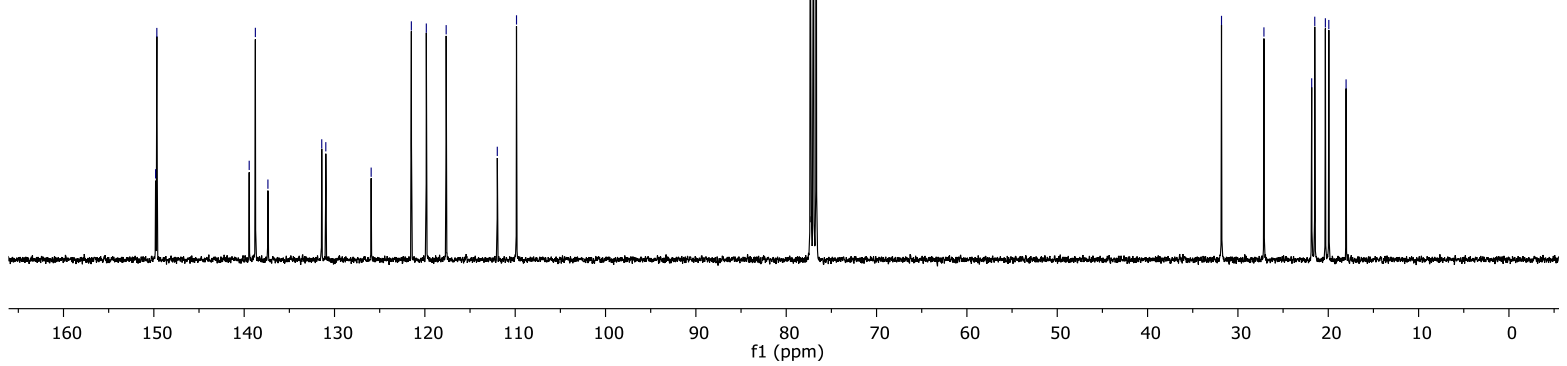


<smiles>[M]C1CCCc2c1n(-c1ccc(C)cn1)c1ccc(OC)cc21</smiles>

2d

$\left(400 \mathrm{MHz}, \mathrm{CDCl}_{3}\right)$

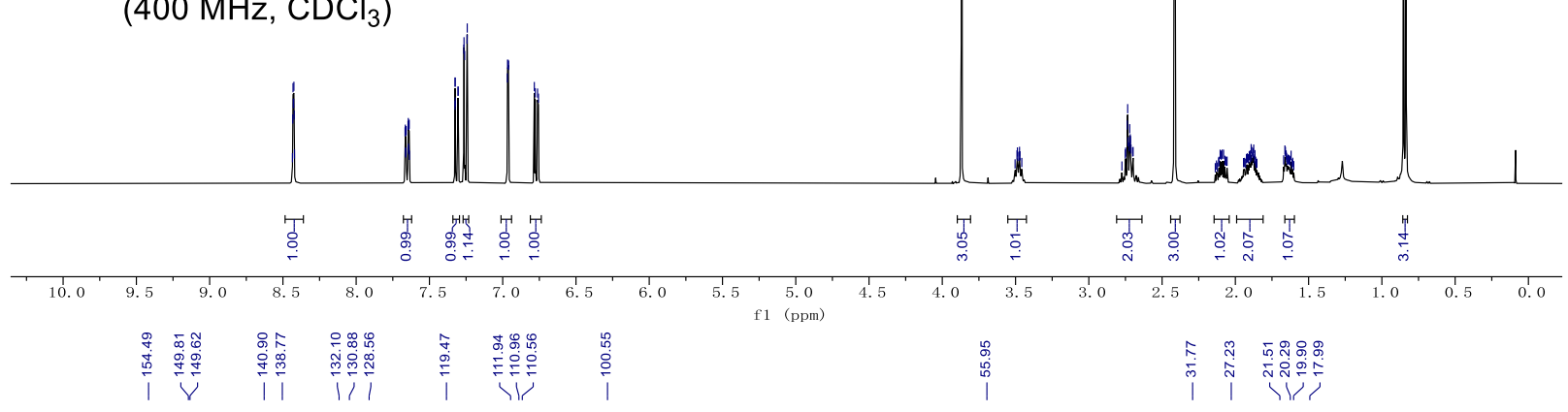

2d

$\left(100 \mathrm{MHz}, \mathrm{CDCl}_{3}\right)$
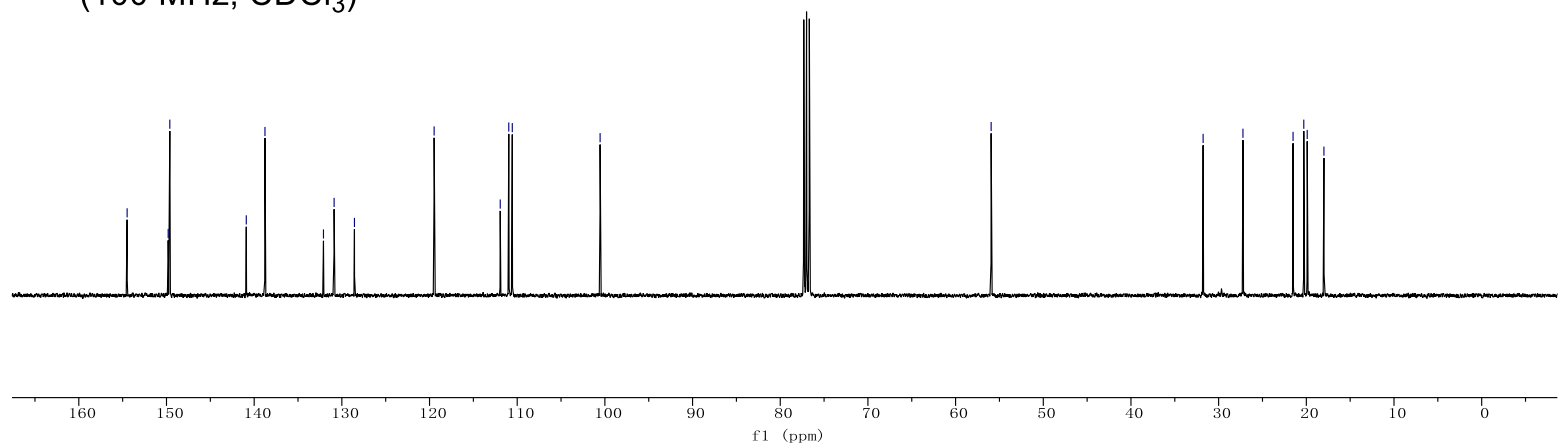

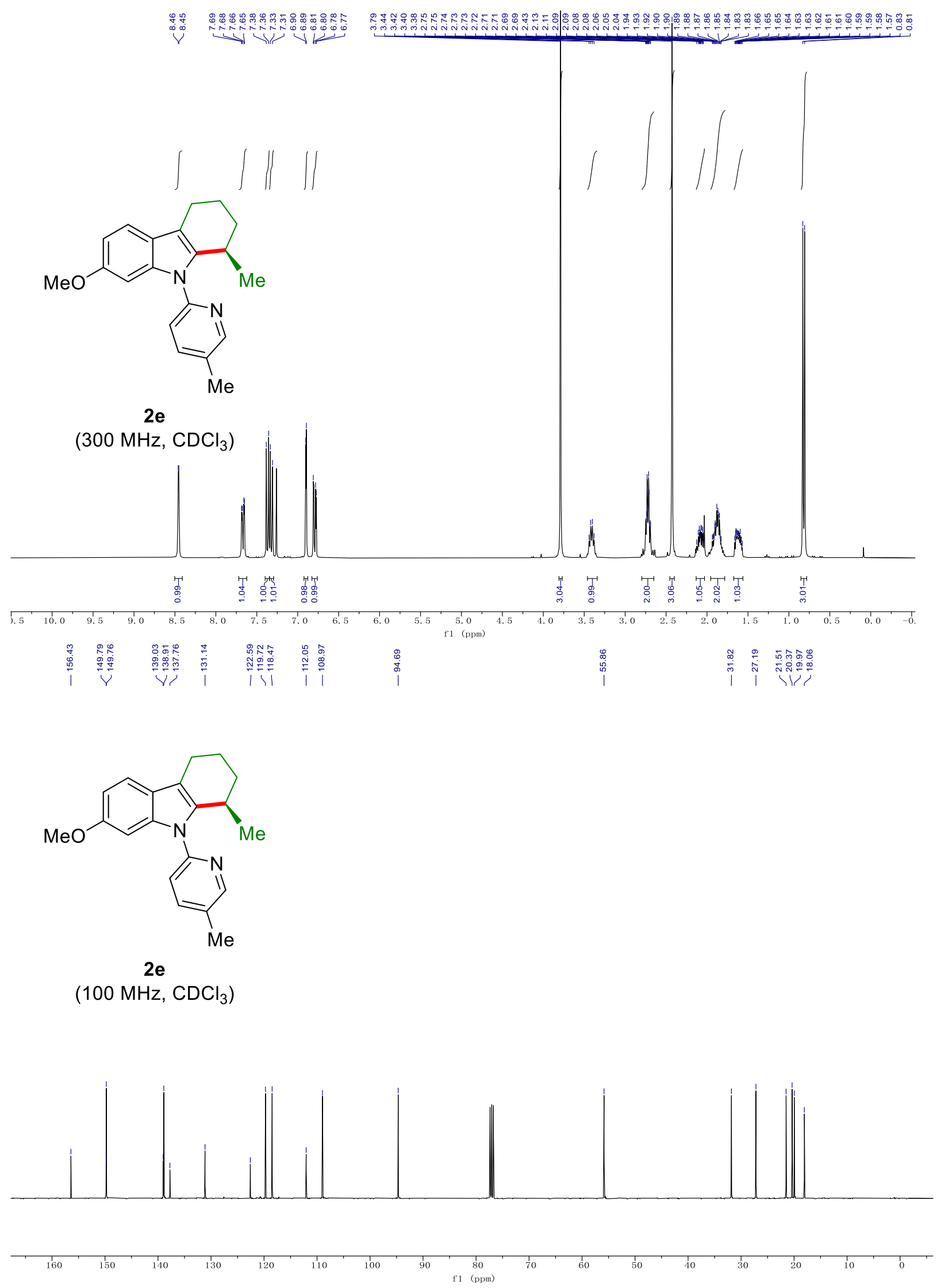


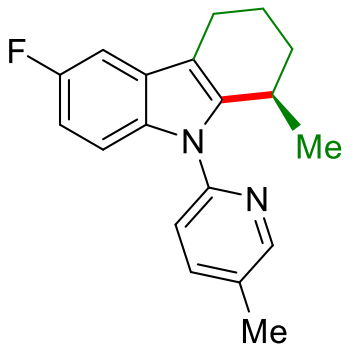

$2 f$

$\left(300 \mathrm{MHz}, \mathrm{CDCl}_{3}\right)$

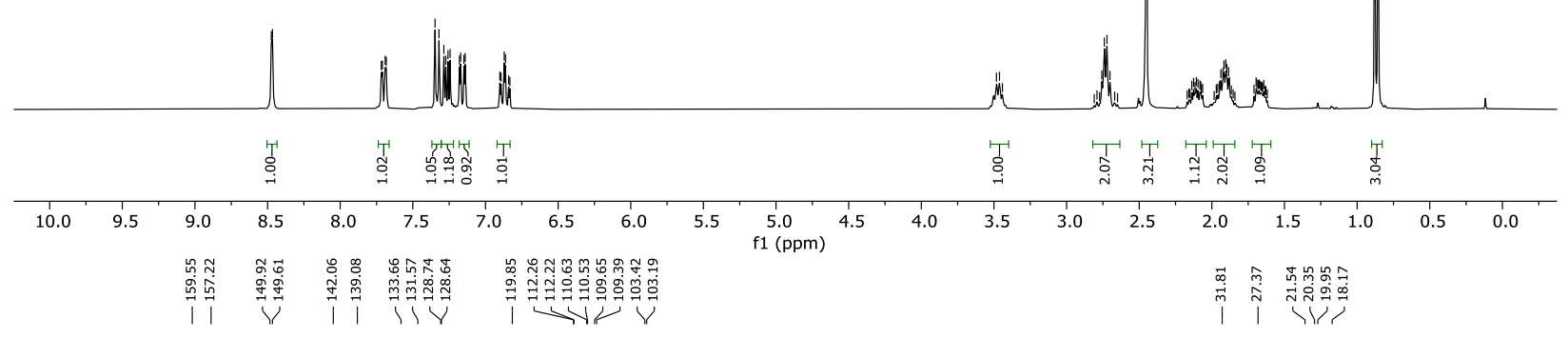<smiles>Cc1ccc(-n2c3c(c4cc(F)ccc42)CCCC3C)nc1</smiles>

$2 f$

$\left(100 \mathrm{MHz}, \mathrm{CDCl}_{3}\right)$

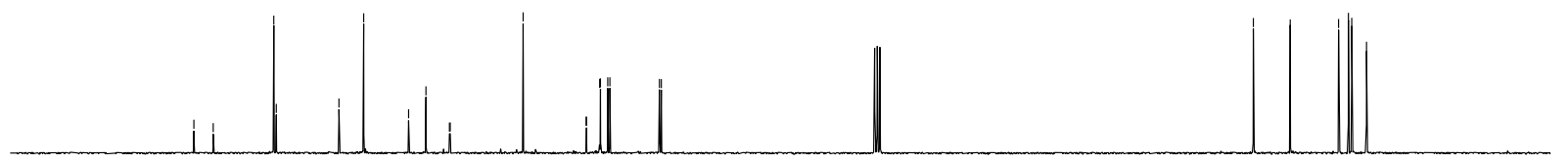

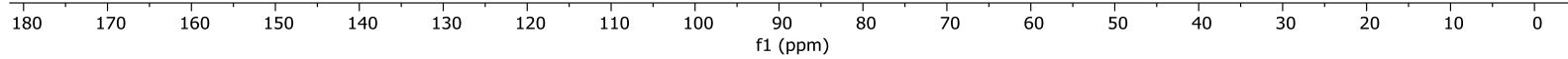




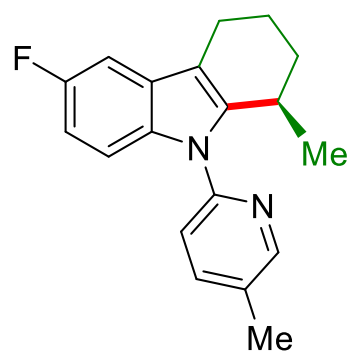

$2 f$

(282 MHz, $\mathrm{CDCl}_{3}$ )

$\begin{array}{lllllllllllllllllllllllllllllllllll}-50 & -55 & -60 & -65 & -70 & -75 & -80 & -85 & -90 & -95 & -100 & -105 & -110 & -115 & -120 & -125 & -130 & -135 & -140 & -145 & -150 & -155 & -160 & -165 & -170 & -175 & -180 & -185 & -190 & -195 & -20\end{array}$ f1 (ppm) 
<smiles>CC1CCCc2c1[nH]c1cc(F)ccc21</smiles><smiles>Cc1ccc(C)nc1</smiles>

$2 \mathrm{~g}$

$\left(400 \mathrm{MHz}, \mathrm{CDCl}_{3}\right.$ )

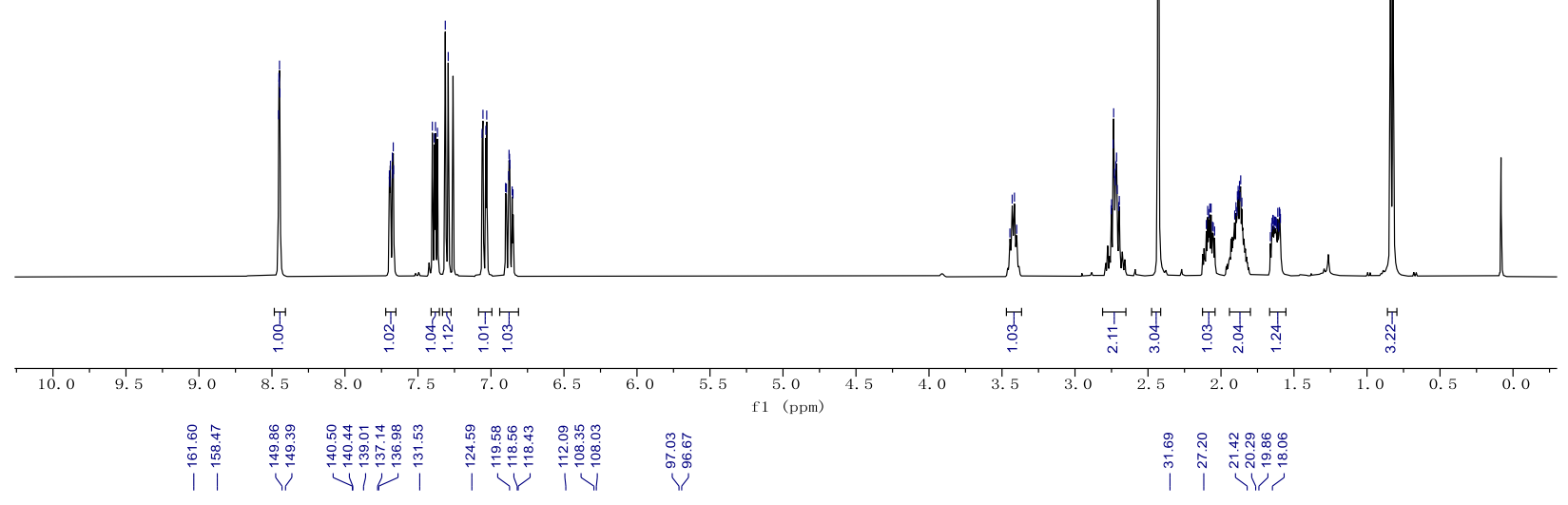<smiles>Cc1ccc(-n2c3c(c4ccc(F)cc42)CCCC3C)nc1</smiles>

2g

(75 $\mathrm{MHz} \mathrm{CDCl}_{3}$ )
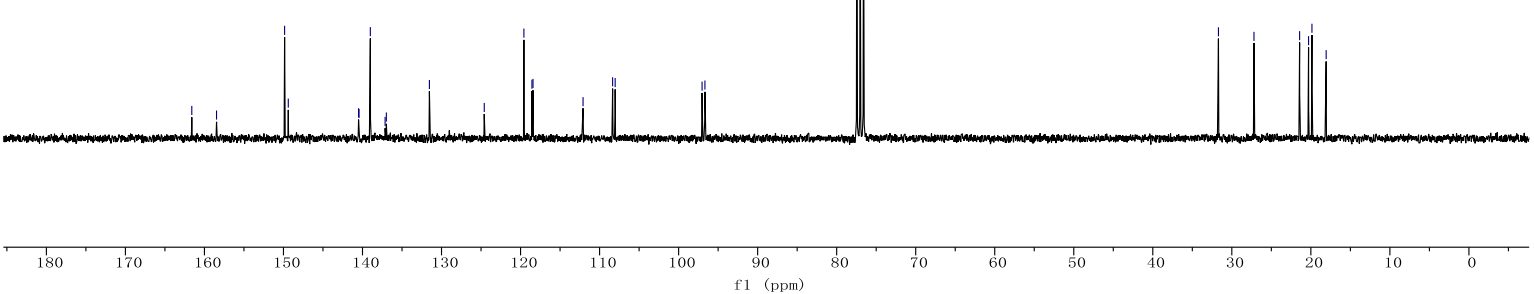


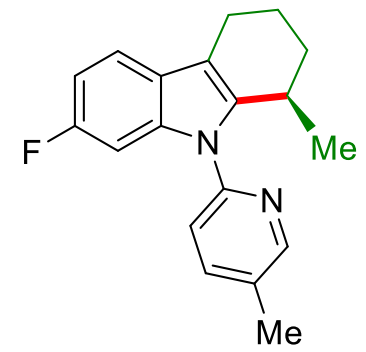

2g

(377 MHz, $\mathrm{CDCl}_{3}$ )

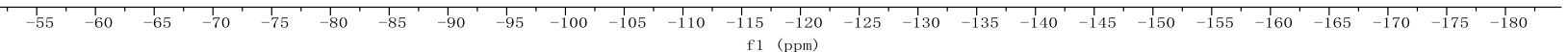


<smiles>Cc1ccc(-n2c3c(c4cc(Cl)ccc42)CCCC3C)nc1</smiles>

$2 \mathrm{~h}$

$$
\text { (400 MHz, } \mathrm{CDCl}_{3} \text { ) }
$$
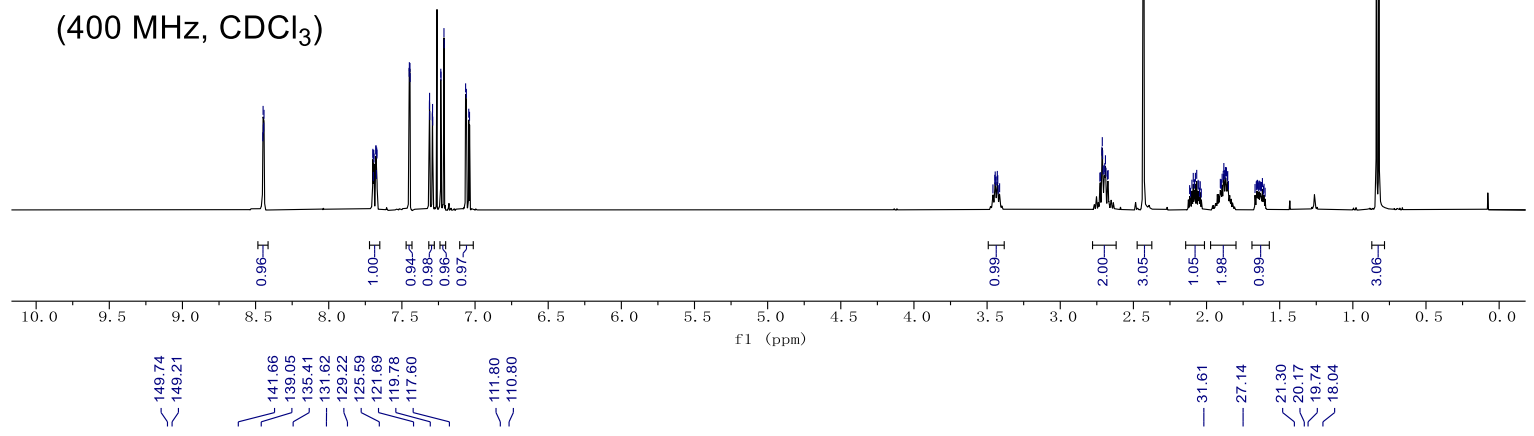<smiles>Cc1ccc(-n2c3c(c4cc(Cl)ccc42)CCCC3C)nc1</smiles>

$2 \mathrm{~h}$

$\left(100 \mathrm{MHz}, \mathrm{CDCl}_{3}\right)$

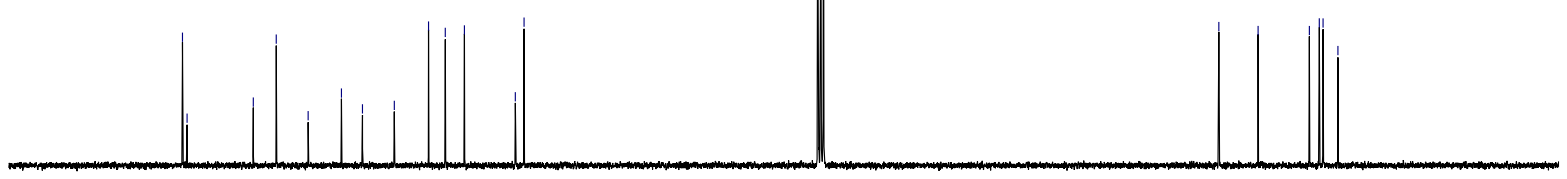



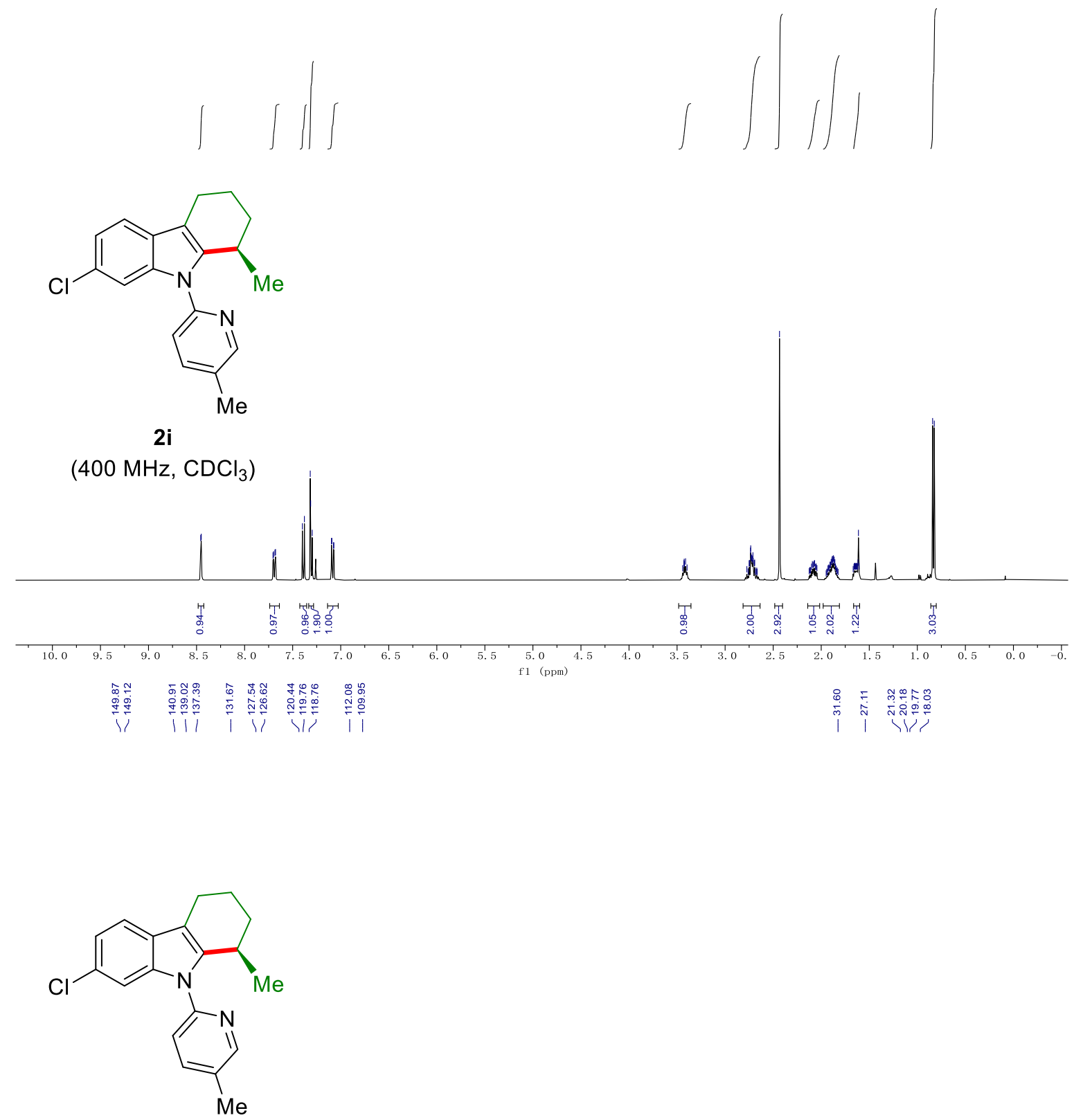

2i

$\left(100 \mathrm{MHz}, \mathrm{CDCl}_{3}\right)$

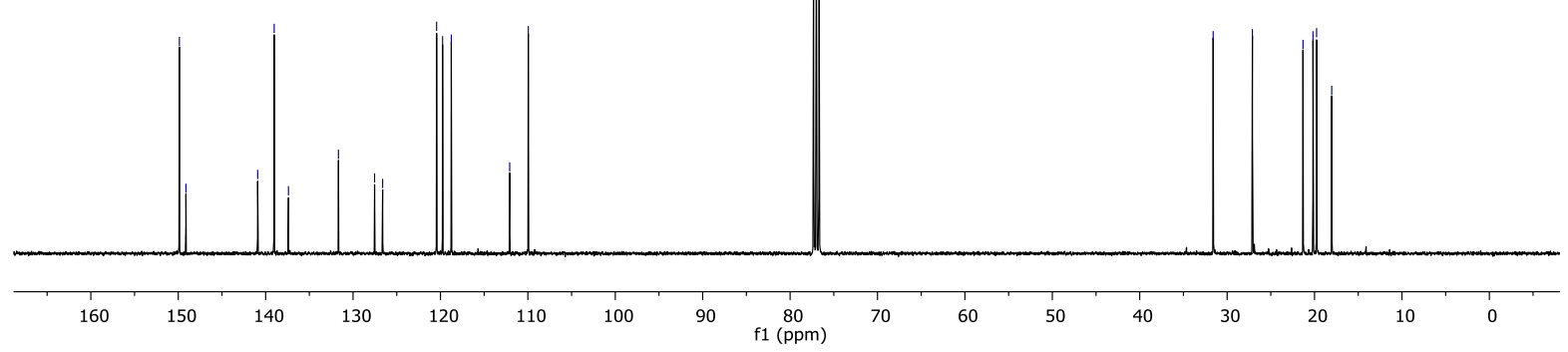



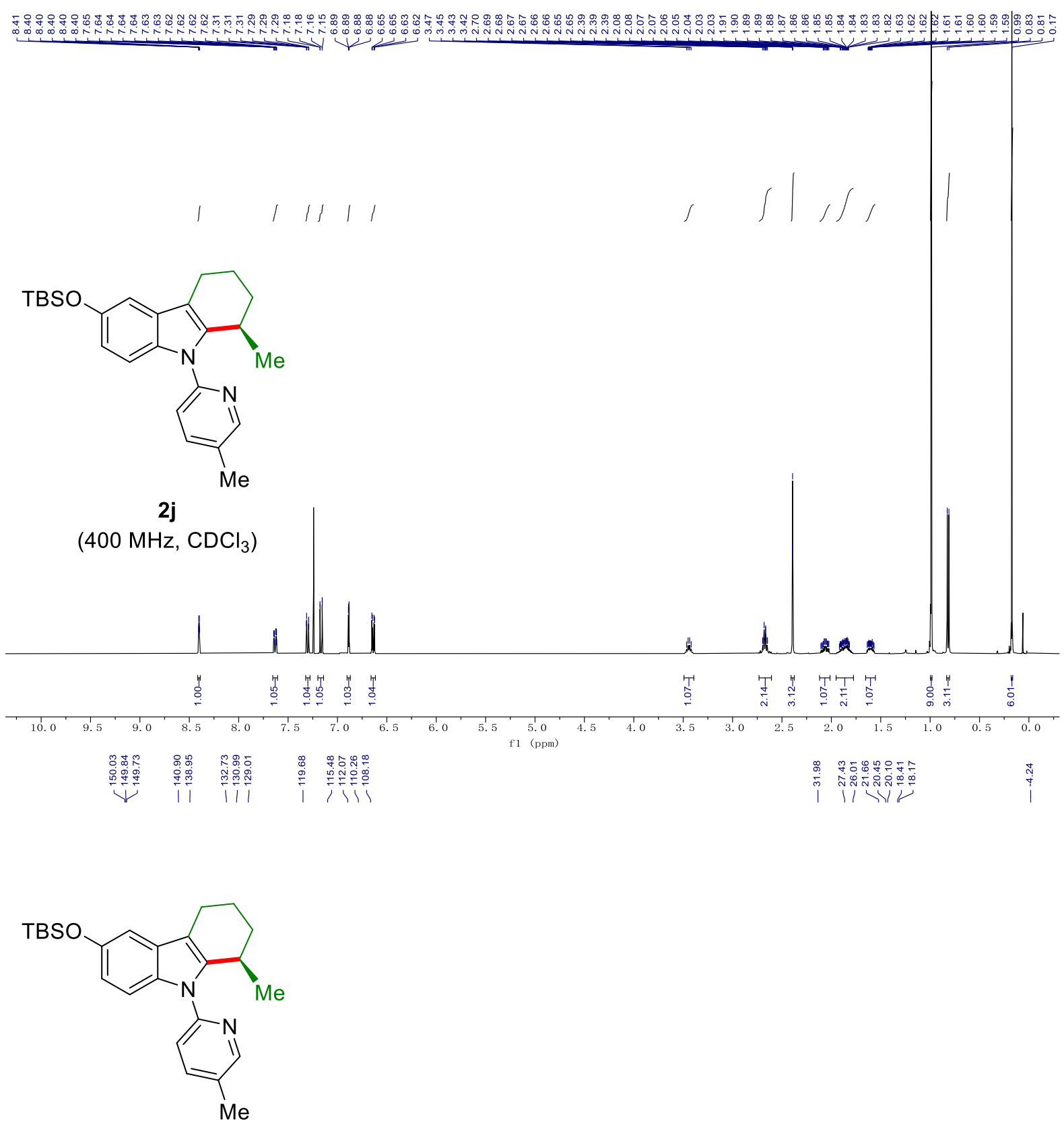

2j

$\left(100 \mathrm{MHz}, \mathrm{CDCl}_{3}\right.$ )
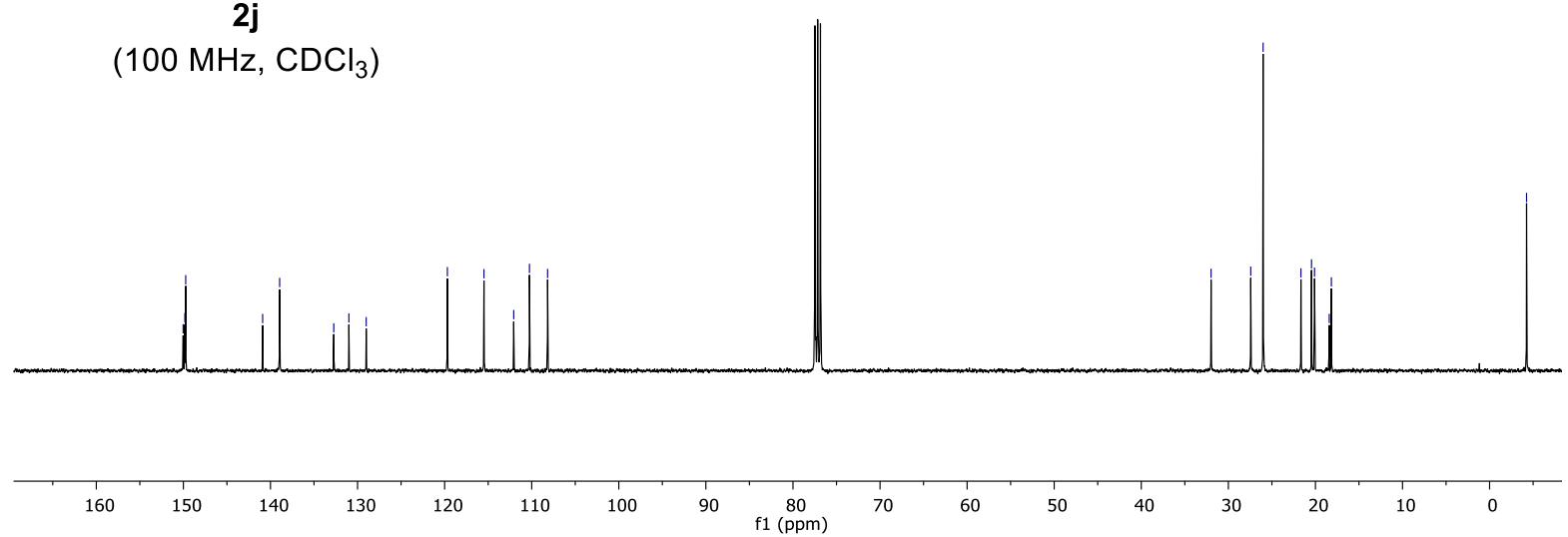

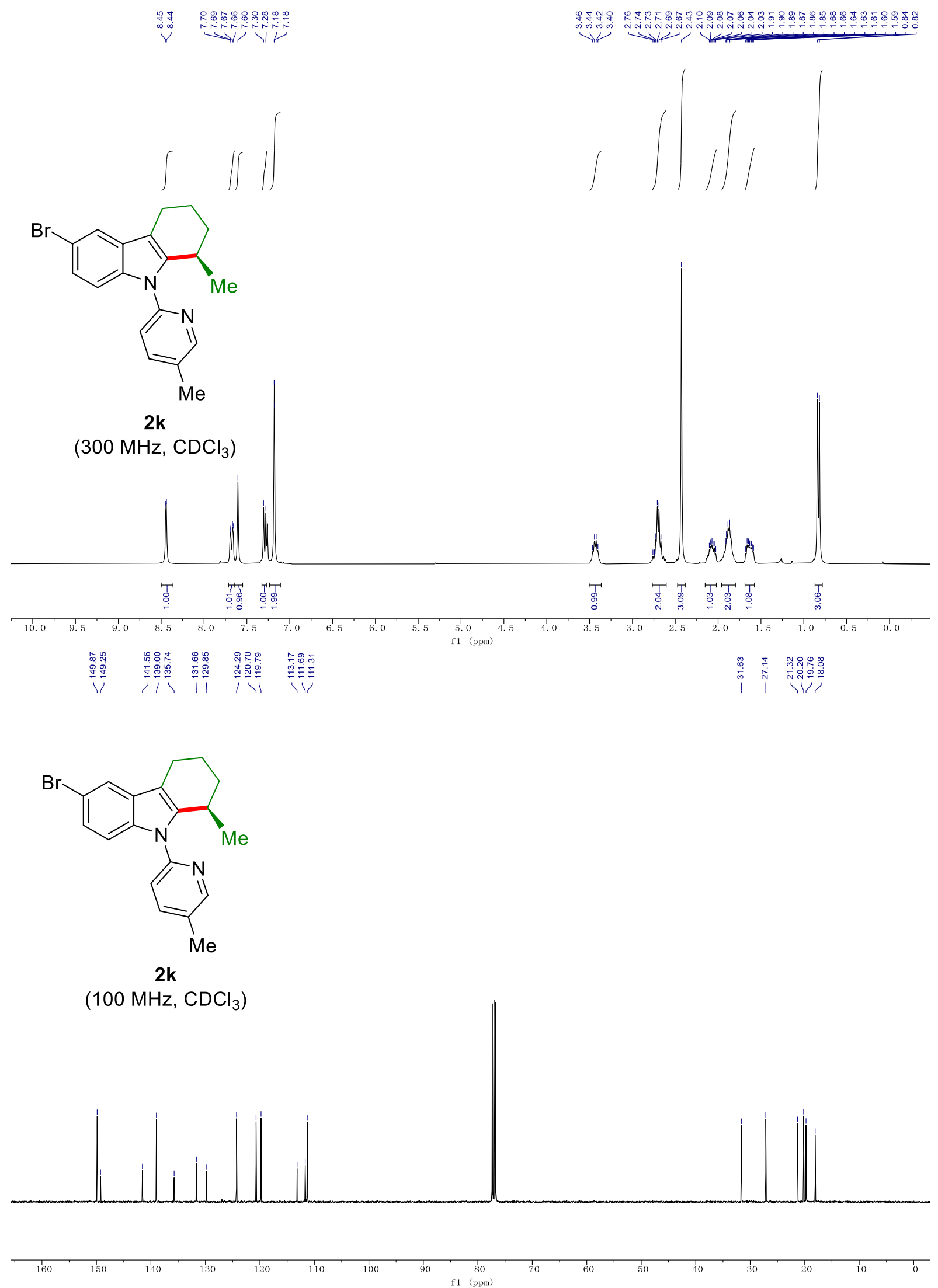

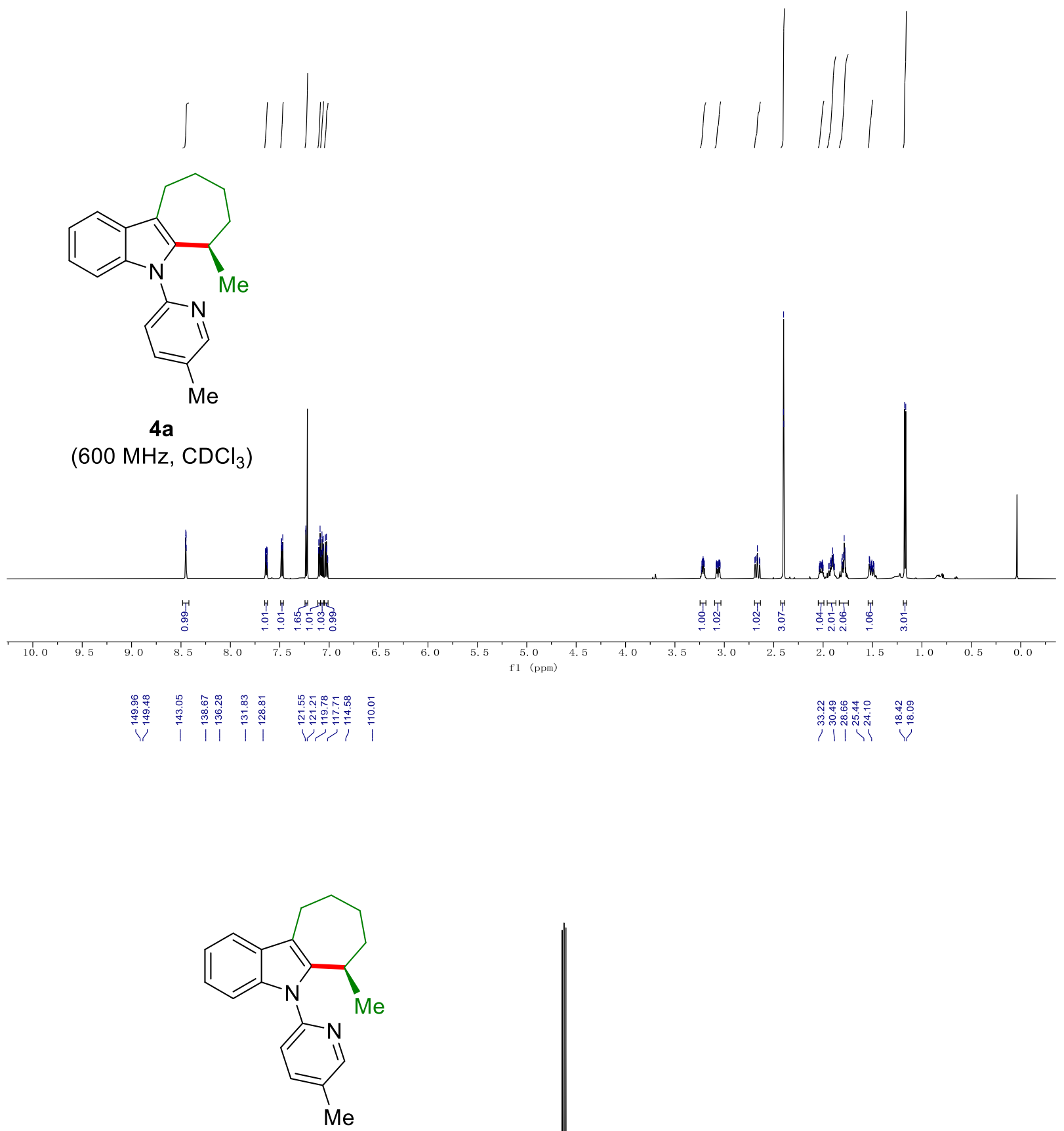

$4 a$

(100 MHz, $\mathrm{CDCl}_{3}$ )
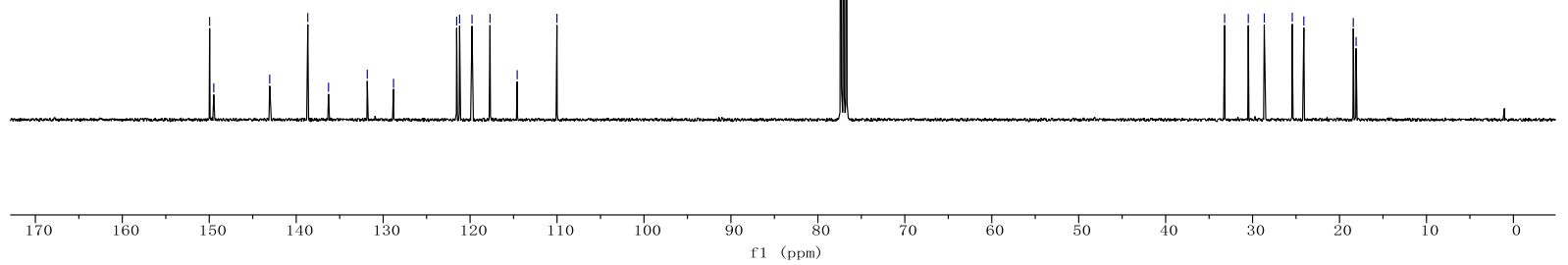


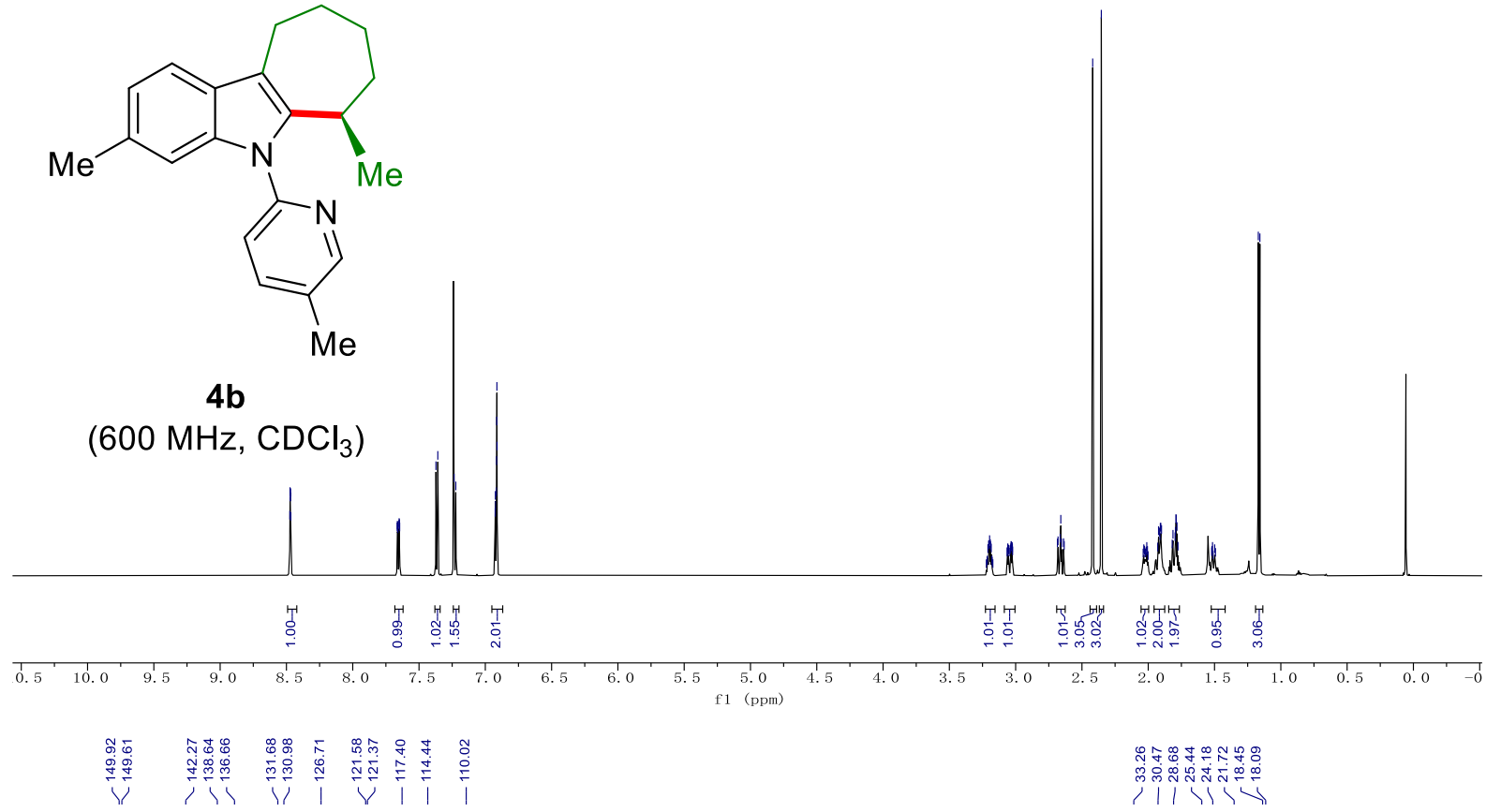<smiles>Cc1ccc(-n2c3c(c4ccc(C)cc42)CCCCC3C)nc1</smiles>

4b

(400 MHz, $\mathrm{CDCl}_{3}$ )

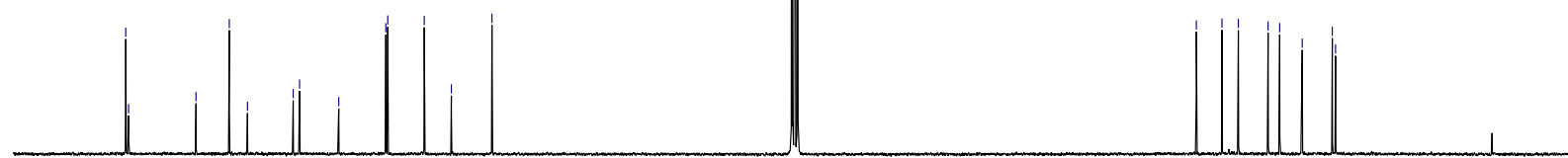

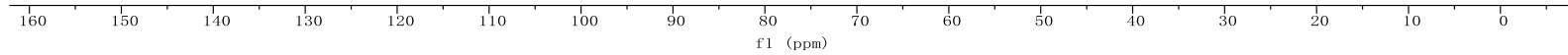


<smiles>Cc1ccc(-n2c3c(c4ccc(Cl)cc42)CCCCC3C)nc1</smiles>

4c

(300 MHz, $\mathrm{CDCl}_{3}$ )

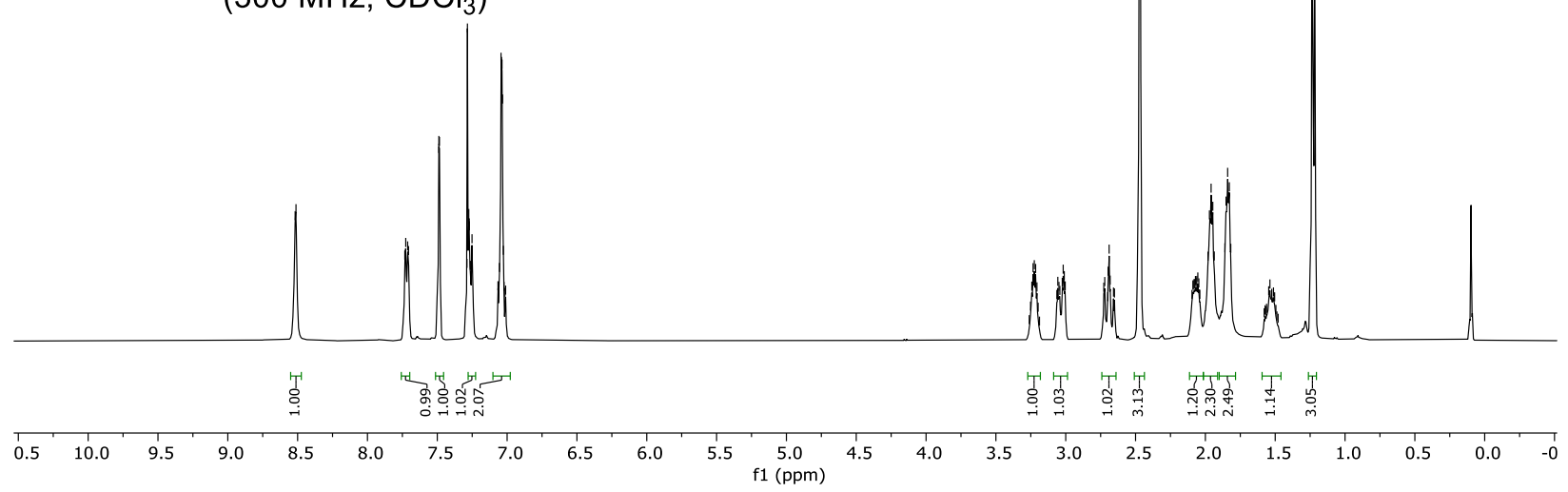

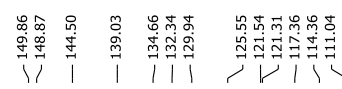

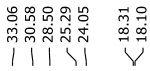

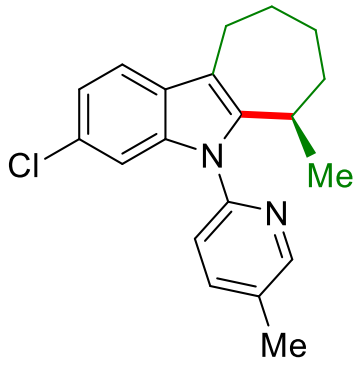

4c

$\left(100 \mathrm{MHz}, \mathrm{CDCl}_{3}\right)$

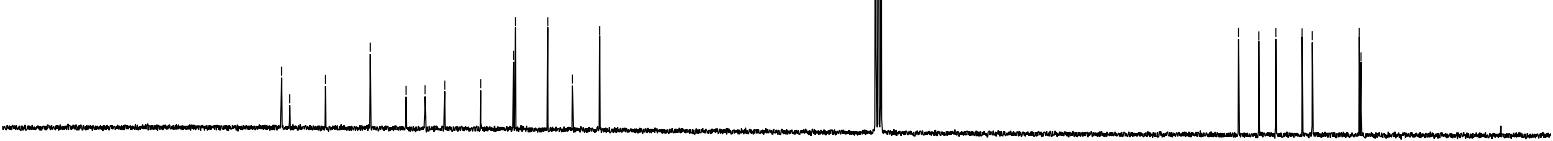

$170 \quad 160$

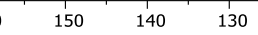

$120 \quad 110$ 